nephropathy, foot ulcer, retinopathy, CAD, PAD, HDL-C (mg/dl) and LDL-C $(\mathrm{mg} / \mathrm{dl})$. The factors which predict the risk of developing ulcer independent of 25(OH) D status were A1c (>6.9\%) [OR 4.37; RR 1.77], HDL-C $(<40 \mathrm{mg} / \mathrm{dl})$ [OR 1.16; RR 1.07], LDL-C (>100mg/dl) [OR 1.07; RR 1.03], triglycerides (>200mg/dl) [OR 1.40; RR 1.19], neuropathy [OR 6.88; RR 3.12], retinopathy [OR 3.34; RR 1.91], hypertension [OR 1.64; RR 1.28], nephropathy [OR 3.12; RR 1.87] \&amp; smoking [OR 4.53; RR 2.99] using odds and risk ratios. In conclusion, it is not clear whether the suppression of delayed wound healing seen during $25(\mathrm{OH})$ $D$ deficiency is due to the secondary effect or is a direct action of vitamin $D$ on certain components of the immune system. Long-term randomized trials are needed to see the impact of vitamin D supplementation on the outcome of diabetic foot patients.

\section{DIABETES EDUCATION}

Guided Audio Tour: Improving Diabetes Prevention and Treatment through Education (Posters: 680-P to 687-P), see page 15.

ก 680-P

Integration and Utilization of Peer Leaders for Long-Term Self-Management Support: Results from Project SEED (Support, Education, and Evaluation in Diabetes)

GRETCHEN PIATT, SHARLENE EMERSON, PATRICIA JOHNSON, DEBRA TILVES, JANICE C. ZGIBOR, Ann Arbor, MI, Pittsburgh, PA

Long-term self-management support (SMS) is critical; however, effective, sustainable models for SMS are scarce. We determined whether a peer leader (PL) SMS model was as effective in achieving and maintaining improvements in glycemic control following a DSME and SMS intervention compared to a traditional DSME support model in 6 rural primary care practices in Pennsylvania. Practices and eligible participants $(n=221$; mean age: 63.0 years, $63.8 \%$ female, $96.8 \%$ Caucasian, $28.5 \%$ at or below poverty level, $32.5 \%$ using insulin, A1c $\geq 7 \%$ : $54.2 \%$ ) were randomized to the intervention (DSME+PL SMS; $n=119$ ) or usual care (UC) group (DSME+traditional DSME support with no $\mathrm{PL} ; \mathrm{n}=102$ ). Each intervention practice had one PL to support participants. Data were collected at baseline, 6 weeks, 6 , and 12 months. Marked decreases in A1c were observed in both groups following DSME (intervention: $-0.24 \%, p<0.0001$; UC: $-0.31 \%, p=0.01$ ); however, as SMS continued over time, intervention participants' average A1c levels continued to significantly decrease at 6 months $(-0.32 \%, \mathrm{p}=0.04)$ and at 12 months $(-0.37 \%, p=0.02)$ in comparison to UC which had increases in average A1c during the support period ( 6 months: $+0.08 \%, p=0.09 ; 12$ months: $+0.02 \%$, $p=0.13$ ). In those who achieved A1c $<7 \%$ following DSME, $94 \%$ and $100 \%$ of participants sustained glycemic control at 6 and 12 months, respectively, in the intervention group. Similarly, in those who achieved $A 1 c<8 \%$ following DSME, $98 \%$ and $96 \%$ of participants sustained glycemic control at 6 and 12 months, respectively, in the intervention group. Results were similar for UC. These data demonstrate that PL SMS is as effective as traditional DSME support in helping participants to maintain glycemic control in the longterm. With the growing prevalence of diabetes and shortage of diabetes educators, it is important to integrate and utilize low-cost interventions in high-risk communities that build on available resources.

Supported by: IDF

\section{ก 681-P}

Socioeconomic and Demographic Predictors of Internet and Social Media Use in Persons With Diabetes

MARCIA A. TESTA, DONALD C. SIMONSON, Boston, MA

Internet and social media tools to access and disseminate health information have increased dramatically; however, there are limited data on their use in persons with diabetes. To help design internet-based technologies to monitor diabetes patients and enhance health messaging, we analyzed two (2008 \& 2010) Pew Research Center cross-sectional telephone surveys. Logistic regression was used to assess the impact of clinical diagnoses, demographic and socioeconomic factors on internet usage. A total of 5,254 individuals participated lage $50 \pm 19 \mathrm{yrs}, 44 \%$ male, 50\% married, $42 \% \mathrm{HS}$ grad or lower, $73 \%$ Caucasian, $41 \%$ full-time employed, $25 \%$ retired, median income $\$ 35,000$ ]. 678 individuals $(13 \%)$ reported having diabetes with comorbidities of hypertension in $67 \%$, heart disease $(24 \%)$, and pulmonary disease $(20 \%)$. Internet usage was reported by $73 \%$ of those with no diabetes and $47 \%$ with diabetes $(p<0.001)$. After adjustment [(Odds Ratio) age in yrs $(O R=0.94)$, male gender $(0.64)$, income $/ \$ 10,000(1.55)$, some college (3.4), Hispanic (0.47), and white race (1.72), all $p<0.0001]$, diabetes $(O R=0.82)$ was no longer a predictor of internet usage $(p=0.12)$. Both crude and adjusted health-related internet use were comparable between diabetes and no diabetes for seeking information on medical problems $(\mathrm{OR}=$ $1.06)$, treatments $(0.88)$ and health care providers (1.07), all $p>0.4$. For all respondents mean \pm SD internet/email use from home [rated 1 (never) - 7 (several times/day)] was $5.4 \pm 0.03$, but 0.3 units lower in persons with diabetes $(p=0.007)$. Internet/email usage from home was more frequent than at work (5.4 vs. 3.8, $p<0.001)$. Effect modification analysis indicated that higher socioeconomic and education status increased internet use more for those with diabetes. Thus, age, education, income, race and ethnicity were important mediators of internet use among patients with diabetes. These factors need to be considered when implementing internet-based diabetes management and education programs.

682-P

Launching a Lifestyle Intervention Program for Diabetes Prevention at the Worksite

M. KAYE KRAMER, KARL K. VANDERWOOD, VINCENT C. ARENA, RACHEL G. MILLER, GERALD L. SCHAFER, ELIZABETH M. VENDITTI, ANDREA M. KRISKA, Pittsburgh, PA

The Group Lifestyle Balance (GLB) program, adapted from the Diabetes Prevention Program's lifestyle intervention, has been shown to reduce risk factors for diabetes and cardiovascular disease (CVD) in several settings. The current project evaluated the effectiveness of GLB program implementation at a large corporate worksite. Study investigators collaborated with worksite representatives to develop and implement GLB program recruitment and intervention strategies. A progressive screening approach using telephone and in-person methods was employed. Adults with a BMl $\geq 24 \mathrm{~kg} / \mathrm{m}^{2}$ and pre-diabetes and/or the metabolic syndrome were eligible to participate. A randomized controlled-delay study design was utilized with two-thirds of subjects randomly assigned to begin the intervention immediately (NOW), and one-third delayed for 6 months (DELAYED). Weight loss and physical activity were assessed at 6 and 12 months from baseline, as were changes in risk factors for diabetes and CVD. A total of 89 individuals enrolled in the study ( $N=60$ NOW, 29 DELAYED). At 6 months the NOW group ( $N=55$ ) demonstrated significantly greater mean weight loss compared to the DELAYED group $(\mathrm{N}=29)(-5.3 \%$ v. $-1 \%, \mathrm{p}<0.001)$, as well as significantly greater improvements in $\mathrm{HbA1c}(-0.1 \%$ v. $-.004 \%, \mathrm{p}=0.01)$ and other diabetes and CVD risk factors. Further, a significantly higher proportion of the NOW group achieved $5 \%$ weight loss than the DELAYED group (46\% v. $7 \%$ ), with a significantly higher proportion of the DELAYED group demonstrating weight gain than the NOW group (7\% v. 45\%). Most importantly, regardless of when they started the intervention, both NOW and DELAYED groups demonstrated significant weight loss $(\sim 5 \%)$ and improvements in diabetes and CVD risk factors after 6 and 12 months of intervention, as well as a significant increase in physical activity levels. These results suggest that the GBL lifestyle intervention can be effectively delivered in a worksite setting and can serve as a potential model for other worksite programs

Supported by: NIH

ก 683-P

Virtual Diabetes Education Improves Resident Physician Knowledge and Performance: A Cluster-Randomized Trial JOANN SPERL-HILLEN, PATRICK J. O'CONNOR, HEIDI EKSTROM, STEVE ASCHE, DEEPA APPANA, PAUL E. JOHNSON, Minneapolis, MN

We conducted a cluster-randomized trial to evaluate the impact of an online virtual diabetes educational experience on resident physician knowledge and ability to care for patients with diabetes mellitus.

Nineteen primary care residency programs with 341 consented residents were randomly assigned to receive $(n=177)$ or not receive $(n=164)$ the intervention. The intervention included 18 virtual learning cases, 3 of which were performed each month for 6 months. Each unique learning case required about 15 minutes to complete using a Web-based interactive electronic health record-like interface that challenged providers to take clinical actions to bring patients to evidence-based care goals within 6 months of simulated time.

Physiologic modeling simulated realistic outcomes of provider actions, and providers received learning feedback designed to critique and guide them between simulated encounters in each learning case. After the intervention period, all residents were assigned a 10-question knowledge test and 4 virtual assessment cases. Generalized linear mixed models were used to test for study-arm differences in knowledge scores and the proportion of residents bringing each assessment case to composite goals for glucose, blood pressure, and lipids. 
A total of 232 residents (97 intervention, 135 control) completed at least 1 case. Residents were $52 \%$ female, $52 \%$ white, mean age $31,44 \%$ family medicine, $53 \%$ internal medicine, and in postgraduate years $1(34 \%), 2$ $(35 \%)$, and 3 and $4(28.5 \%)$. The proportion of residents bringing patients to composite goal using case-specific pre-determined appropriate and safe treatment criteria was significantly higher in the intervention group than in controls: case $1: 21.2 \%$ vs. $1.8 \%, P=.002$; case $2: 15.7 \%$ vs. $4.7 \%, P=.02$; case 3: $48.0 \%$ vs. $10.4 \%, P<.001$; case $4: 42.1 \%$ vs. $18.7 \%, P=.002$.

Diabetes simulation-based training significantly improved resident knowledge scores and ability to achieve care goals using virtual patients.

Health Education Disparities in Diabetic Subjects

ก 684-P JANELLE BRANOFF, SADIA HUMA, CHLOE REY, MICHAEL JIROUTEK, BETH SUTTON, Buies Creek, NC

Previous studies in diabetes show disparities in receipt of health education. The American Diabetes Association (ADA) has determined standard of care guidelines for the treatment of diabetes, but it remains unclear if there has been any recent improvement in the health education provided to diabetic patients. The purpose of this study was to identify disparities in diabetes health education using the most recently available data in the National Ambulatory Medical Care Survey (NAMCS). This retrospective, cross-sectional, observational study examined data from the 2008 - 2010 NAMCS database on adults $\geq 18$ years with a primary diagnosis of diabetes. Weighted chi-square analyses were conducted to identify associations between diet/nutrition, exercise, and weight reduction education and each of the following variables: sex, race, ethnicity, tobacco use, age, physician location, metropolitan setting, provider type, physician specialty, and insurance type. A weighted multivariate logistic regression model was also constructed. Among patients included in this study $(n=2,285), 32.5 \%$ received diet/nutrition education, $28.4 \%$ received exercise education, and $12.3 \%$ received weight reduction education. Patients with $\mathrm{BMI} \leq 25(\mathrm{OR}=$ $\left.0.53, \mathrm{Cl}_{95} 0.32-0.87\right)$, Medicaid recipients $(0.61,0.39-0.96)$, and office visits in a non-metropolitan area $(0.48,0.24-0.95)$ were significantly less likely to receive nutrition education. Current tobacco users $(0.54,0.29-0.96)$, as well as Medicaid recipients $(0.59,0.31-0.89)$ and 'other' payment type [self-pay, worker's compensation, no charge and Other] $(0.58,0.35-0.96)$ were significantly less likely to receive exercise education. Patients with a BMI $\leq 25(0.12,0.06-0.25)$ and office visits in the West $(0.27,0.11-0.65)$ or Midwest $(0.44,0.22-0.85)$ were significantly less likely to receive weight reduction education. Our findings indicate that health education disparities in diabetes still exist, suggesting patients may not be receiving the necessary information from their physicians.

\section{5-P}

Use of Diabetes Clinical Guidelines by Physicians and Relationship to their Knowledge and Practice Patterns

MARK D. CORRIERE, FRED BRANCATI, STEPHEN SISSON, LAURA MINANG, RITA KALYANI, Baltimore, MD

Consensus guidelines exist for management of type 2 diabetes (DM2) (e.g., ADA). How often guidelines are used in practice by health care providers and the relationship to their diabetes knowledge and practice patterns has not been fully described. We examined responses from 385 community physicians who completed an online survey. A diabetes knowledge score was calculated for 5 questions related to prevention of diabetes complications and glycemic goals. We explored the association of clinical guideline use with knowledge and practice patterns using multiple logistic regression. $53 \%$ of participants used diabetes clinical guidelines routinely in their practice. Guideline users (GU) vs. non-guideline users (NGU) included similar proportions of primary care physicians ( $56 \%$ vs. $47 \%, p=0.065$ ) but more endocrinologists $(7.7 \%$ vs. $2.2 \%, p=0.01)$ and less "other" subspecialists $(36.1 \%$ vs. $51.1 \%, p=0.003)$ Despite similar practice volumes, GU more frequently diagnosed DM2 than NGU (37.1\% vs. 22.8\%, $p=0.002)$. GU mean diabetes knowledge score was significantly higher than NGU $(3.37 \pm 0.072$ vs. $2.76 \pm 0.084, p<0.001)$. GU were also significantly more likely to report a good understanding of DM2 medications (OR=2.99, 95\% Cl 1.95-4.61, $\mathrm{p}<0.001)$ and use lifestyle and metformin combination as first-line treatment for DM2 (OR=1.73, 1.112.70, $p=0.015$ ). NGU were more likely to report unfamiliarity with insulin as a barrier to early use $(\mathrm{OR}=2.43,1.26-4.72, \mathrm{p}=0.007)$ and unfamiliarity with pharmacologic options as a barrier to intensive multifactorial interventions in their practice $(\mathrm{OR}=3.16,1.72-5.80, \mathrm{p}<0.001)$. All associations were independent of specialty, practice volume, and number of diabetes patients in adjusted models. Guideline use is associated with better diabetes-related knowledge and practice patterns. Future educational interventions that emphasize use of diabetes clinical guidelines by providers and improve knowledge of pharmacological therapies are needed.

\section{ก 686-P}

Factors Influencing Uptake of Structured Education for Type 1 Diabetes and Long-Term Outcomes in an Urban Clinic Population; Impact of Ethnicity and Social Deprivation

DAVID HOPKINS, ROLAND FOXCROFT, DANIEL SIMPSON, AMANDA SIMONDS, PENNY JACKSON, SIOBHAN PENDER, STEPHANIE A. AMIEL, London, United Kingdom, Crawley, United Kingdom

Structured education on flexible insulin therapy is recommended by national guidelines for the management of type 1 diabetes (T1D) in the UK, and is provided at no direct cost to patients. Despite this, uptake of training remains suboptimal. To determine the factors influencing this, we have conducted a retrospective analysis of combined biochemical, demographic and health resource utilisation data collected over a 10 year period for a cohort of T1D patients ( $n=1365$ ) resident in a geographically defined urban area, who attended one of two specialist diabetes clinics offering the DAFNE (Dose Adjustment For Normal Eating) structured education program.

$405(29.7 \%)$ of the cohort attended DAFNE between 2001 and 2012. There were no differences in mean $( \pm S D)$ age $(37 \pm 11$ yrs vs $38 \pm 13 \mathrm{yrs}, p=0.05)$ duration of diabetes $(15+12$ yrs vs $14+11$ yrs, $p=0.2)$ or baseline $H b A 1 c(8.7 \pm 1.8 \%$ vs $8.6 \pm 2.0 \%, p=0.5$ ) between those who received training and those who did not. However, ethnic minorities were under-represented among those attending DAFNE $(21.2 \%$ vs $30.5 \%, \mathrm{p}<0.001)$ as were individuals residing in more socially deprived districts (mean Index of Multiple Deprivation score 27.2 \pm 9.8 DAFNE vs $30.3 \pm 9.6$ others, $p<0.001$ ).

Those who attended DAFNE achieved significantly better long-term glycemic control (mean HbA1c at study end $8.1 \pm 1.2$ vs $8.4 \pm 1.8 \%, p=0.001$, mean follow-up post-DAFNE 4.8 years ) and a lower prevalence of severe hypoglycemia requiring hospitalisation (1.7 vs $4.8 \%, p=0.006)$. The association between DAFNE and improved glycemic control persisted after controlling for social deprivation score.These data indicate significant inequalities in access to optimal diabetes management, impacting on subsequent outcomes. Improving uptake by patients from ethnic minority and poor socio-economic backgrounds could have economic benefits through improved glycemia and reduced need for emergency diabetes care.

Supported by: Novo Nordisk, Inc.

\section{Effectiveness of a Multicultural Community Program}

ก 687-P MELINDA D. MARYNIUK, SARAH IMERSHEIN, MATTHEW VERLINDEN, GIPPER MONSON, RICHARD A. JACKSON, Boston, MA, Bridgewater, NJ

Reaching underserved populations with cost-effective programs that improve outcomes for people with diabetes and pre-diabetes remains a challenge. We report results of a successful pilot implemented in 4 ethnically diverse cities over 3 months reaching 369 participants. The objectives were to reach underserved populations, engage them in the healthcare system, increase awareness of key diabetes tests, and measure changes in biomarkers and healthy behaviors. The intervention included a 1-hour baseline class on understanding key diabetes tests plus collection of data (surveys, blood pressure (BP), and point-of-care A1C) The 3-month follow-up class included re-collection of data and a 1-hour class on healthy eating.

Four community liaisons (CLs) (individuals familiar with the target communities but not previously trained in health education) recruited participants and delivered the program. The CLs completed two 4-hour training sessions on program delivery and were supported with periodic team phone calls. Baseline data revealed the target population was reached $160 \%$ with annual household income $<\$ 25,000,45 \%$ with high school diploma or less education, $21 \%$ of participants with diabetes had no medical visit in $>6$ months, 34\% Hispanic, 28\% African American). Most participants $(67 \%)$ had elevated $B P \geq 130 / 80$ and $58 \%$ of participants with diabetes had an $\mathrm{A} 1 \mathrm{C} \geq 7$.

Data collected on 245 individuals at follow-up revealed improvement in mean $\mathrm{A} 1 \mathrm{C}$ for participants with diabetes (7.53 to $7.26 \mathrm{p}=0.04)$, with a greater improvement if baseline $A 1 C \geq 7$ ( 8.39 to $7.85, p=0.011$ ). People with pre-diabetes or undiagnosed diabetes (responded "no diabetes" but had a baseline $A 1 C \geq 5.7$ ) also showed improvement in mean $A 1 C$ (6.14 to $5.74, p<.0005)$. Mean systolic BP improved from 151.3 to $144.5, p=.002$ in all participants with a baseline systolic $B P \geq 130$. Improvements were reported in lifestyle behaviors, especially in those with pre-diabetes. Brief, low-cost community interventions can have significant impact on improving diabetes risk factors. 


\section{DIABETES EDUCATION}

\section{Text Messaging Engages Adolescents With Type 1 Diabetes in their} Care

AJAY SINGHVI, SARAH SALAMATI, BERN SHEN, EVA TSALIKIAN, Iowa City, IA

The management of adolescents with Type 1 Diabetes (T1D) is challenging. Previous interventions including paper and digital glucose records have had limited success. The advent of text messaging (SMS) technology among adolescents offers a new approach. According to a recent survey, threefourths of teens own a cell phone and two-thirds text daily a median of 60 messages.

This pilot study included adolescents with T1D, ages 14-19 years, with past 3 month $\mathrm{HbA1C}>8 \%$, who had SMS-enabled cell phone. Eighty six subjects were recruited and randomized into one of two groups: 46 patients into the text messaging group (TMG) and 40 patients into the standard care group (SCG). The TMG received 4 daily messages asking them to text back pre-breakfast, -lunch, -dinner and -bedtime glucose readings. Replies were automatically parsed, and branching logic dictated a clinically appropriate response depending on whether values were low, normal or high. Self-reported glucose values were also automatically graphed and displayed by an intelligent, secure, cloud-based bidirectional SMS platform (HealthCrowd) on both population and individual patient dashboards. This platform also included a real-time "feed" of outgoing and incoming text messages, automatically triggered alerts, and overall program statistics. $\mathrm{HbA1C}$ and questionnaires regarding satisfaction with the program were collected at 3 and 6 months for both the SCG and TMG.

Data have been collected from 43 patients (93.4\%) in TMG and 34 patients (84\%) in SCG at 3 months and 25 patients (54.3\%) and 19 patients (47.5\%) in each group respectively at 6 months. At 3 months, HbA1c decreased from $9.62 \%$ to $9.3 \%$ in TMG, but little change was seen in SCG $19.2 \%$ to $9.3 \%$ ). Measures of satisfaction and self-efficacy were high according to the questionnaire responses.

This study demonstrates feasibility of an innovative method to engage adolescents with T1D in their own care. Future studies will be needed to determine whether SMS technology has a sustained benefit on management of T1D in adolescents.

Supported by: University of lowa

689-P

Achieving Normal Glycemic Control by Implementing a Diabetes Prevention Program for Non-Complicated Type II Diabetics

JAN V. CHACON, RAMIN NADERI, San Jose, CA

Indian Health Center of Santa Clara Valley (IHC) has implemented the Diabetes Prevention Program (DPP) since 2004. The curriculum is based on a study sponsored by the National Institute of Health $(\mathrm{NIH})$ which found that an intensive lifestyle intervention can lower the risk of diabetes by $58 \%$. Because American Indians have the highest rates of diabetes in the world, in 2004 the Indian Health Service funded DPP pilot programs across the country to translate the $\mathrm{NIH}$ study from research to community settings. IHC's DPP is an innovative 17-week lifestyle change program taught by a multidisciplinary team of professionals including a Registered Dietitian, Certified Diabetes Educator, Mental Health Counselor, Kinesiologist, Fitness Trainers and Health Educators. Baseline/follow up labs, matrix and surveys are obtained to track outcomes. IHC's DPP has an annual diabetes conversion rate of $1 \%$ as compared to the $\mathrm{NIH}$ study predicted rate of $11 \%$ without the intervention. Due to the tremendous success of DPP, in $2007 \mathrm{IHC}$ started pursuing funding to expand to other populations. In 2010, IHC contracted with a Santa Clara County Public Health Sector insurance plan to provide DPP to participants with pre-diabetes with a Fasting Blood Glucose (FBG) of 100-125 as well as non-complicated Type II diabetes. Patients with a FBG $>125$ and no complications were referred by their primary care physicians. Of 32 participants, 18 were diagnosed with Type II diabetes. Follow up labs indicate that after the intensive intervention, $44 \%$ of diabetic participants decreased their FBG from diabetic range to pre-diabetic range $(<125)$, and $5 \%$ decreased to normal glycemic range $(<100)$. All participants showed increased motivation to become active in their management of diabetes risk factors. Using DPP concepts, participants with non-complicated diabetes were able to lower their risk of diabetes complications and increase their quality of life. This can also translate into healthcare cost savings.
$690-P$

Organization Program of DiabEtes INsulIN ManaGement (OPENING) in Patients With T2DM: A 16-week, Multicenter, Open-Label, Controlled, Randomized and Prospective Study

XIAOHUI GUO, LINONG JI, JIE LIU, JUMING LU, Beijing, China, Taiyuan, China

Background: Organization Program of DiabEtes INsullN ManaGement (OPENING) was designed to evaluate the effectiveness of structured education in patients with T2DM

Objective and Design: Enrolled patients were over 18 years old and inadequately controlled $(\mathrm{HbA} 1 \mathrm{c} \%>7.5 \%)$ with two or more $\mathrm{OADs}$ for over 3 months.Patients were randomized (1:1) to the interventional group (structured education plus insulin therapy) or the control group (usual care plus insulin therapy). Subjects in both groups were treated by the Protamine Recombinant Human Insulin Injection (Scilin M30) twice daily. In addition, the subjects in interventional group were educated with contents of seven blocks (including medications, insulin injection technique, BG monitoring, diet, physical activity, preventing hypoglycemia events and preventing complications)

Results: 1511 patients from 48 centers were enrolled in China. 1289 patients were completed the study, 646 were in interventional group and 643 in control group. After 16-week treatment, the reduction of mean $\mathrm{HbA1c}$ was $2.17 \%$ in interventional group and $2.07 \%$ in control group; After removing the influence of $\mathrm{HbA1c}$ baseline value by covariance analysis, the $\mathrm{HbA1c}$ reduction in interventional group was $0.16 \%$ larger than control group $(\mathrm{p}<0.01)$; The proportion of patients who achieved target $\mathrm{HbA1C}$ of less than $7 \%$ was $43.81 \%$ and $36.86 \%$,respectively $(p<0.05)$; the incidence of hypoglycemia events was $29.57 \%$ and $24.26 \%$, respectively,but most of which were mild or moderate. The treatment satisfaction scores of patients continued to rise throughout the treatment in interventional group, and the treatment satisfaction score was 6.37 at the endpoint (the full score was 7). Conclusions: The OPENING could further enhance the efficacy of insulin treatment, and the patients had an excellent satisfaction with the OPENING throughout the treatment.

691-P

Development of a Clinically Meaningful Measure of Carbohydrate Counting Skills in Patients With Diabetes: Carbohydrate Counting Competency Index (C3i)

IAIN CRANSTON, RALPH ZIEGLER, DAVID A. CAVAN, KATHARINE BARNARD, CHRISTOPHER PARKIN, WALTER KOEHLER, IRIS VESPER, MATTHIAS A. SCHWEITZER, ROBIN S. WAGNER, Portsmouth, United Kingdom, Muenster, Germany, Bournemouth, United Kingdom, Southampton, United Kingdom, Boulder City, NV, Mannheim, Germany, Indianapolis, IN

Carbohydrate $(\mathrm{CHO})$ counting is a core skill for patients with diabetes who wish to practice effective meal-bolus insulin therapy. While the principles are straightforward, applying this skill reliably over diverse meals is challenging, and clinicians' ability to identify patients who have difficulties with $\mathrm{CHO}$ counting is often suboptimal. We developed a metric to assess and compare $\mathrm{CHO}$ skills in patients enrolled in the Automated Bolus Advisor Control and Usability Study (ABACUS), a 26-week, prospective, randomized, controlled, multi-national study of poorly controlled patients treated with multiple daily insulin injection (MDI) therapy, with mean (SD) baseline A1C $8.9(1.2) \%$. At baseline and study end, patients were asked to provide $\mathrm{CHO}$ estimates for 10 photographic standard meal plates (DAFNE) of known $\mathrm{CHO}$ content. Accuracy of $\mathrm{CHO}$ estimation was assessed using the mean of meal errors between true and estimated values (MME). Variability was assessed using the mean of meal errors between true and estimated values (MMAE). The estimates were calculated into a single metric: $\mathrm{CHO}$ Counting Competency index (C3i). The C3i is expressed numerically (MME : MMAE) or visually plotted on a scale as MME \pm MMAE. (Figure 1) This index provides information that allows clinicians to identify and address $\mathrm{CHO}$ counting deficits. The $\mathrm{C} 3 \mathrm{i}$ can be used to evaluate change in patient $\mathrm{CHO}$ competency over time and for research purposes. 
Figure. Examples of $\mathrm{C} 3 \mathrm{i}$ assessments

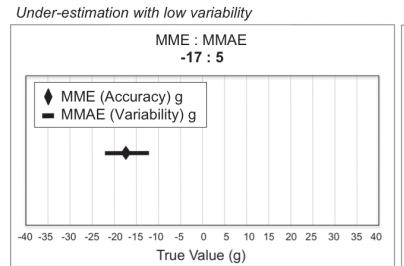

Supported by: Roche Diagnostics GmbH

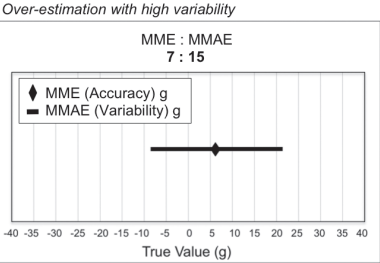

692-P

Self-Monitoring of Blood Glucose in Type 1 Diabetes: Practice Frequency and Correlation With Glycemic Control in a Chinese Adult Sample

ZHAOYI OIN, JINHUA YAN, DAIZHI YANG, JIN LI, HONGRONG DENG, LI LIU, BIN YAO, JIANPING WENG, Guangzhou, China

This study aimed to survey practice of SMBG in adult patients with type 1 diabetes (T1D) in Guangdong Province of China, to correlate the frequency of SMBG to HbA1c, and to explore factors that might affect the association.

The multi-center study consecutively enrolled 210 non-newly-diagnosed patients with T1D visiting 11 diabetes clinics across Guangdong during the period of January to March 2012 [female/male:126/84; age: 30.0 (18-78) yrs; diabetes duration: 4.75 (0.5-35) yrs; HbA1c: $8.5 \pm 2.6 \%$ ]. Self-reported frequency of SMBG was recorded. HbA1c was measured locally by a uniform and qualified point-of-care testing system.

Of the sample, $47.6 \%$ tested less often than once daily. Median frequency of daily SMBG was 0.71 (0-7.00). Linear correlation analysis showed a negative correlation between SMBG frequency and HbA1c (Spearman rank correlation $F=0.352, P<0.001)$. The general negative correlation was then put under examination in different groups of patients, i.e. patients with different education levels, insulin regimens, insulin use experience or insulin doses, and patients in or out of the $\mathrm{HbA1c}$ target range, as shown in table 1.

In conclusion, SMBG practice in adult patients with T1D in Guangdong is suboptimal. More frequent SMBG is associated with lower HbA1c and the association is more prominent in patients with higher education levels, using MDI regimen, with diabetes duration over 1 year, or with HbA1c out of target range.

Table 1. Correlations between SMBG frequency and $\mathrm{HbA} 1 \mathrm{c}$ stratified by education level, insulin regimen, diabetes duration, insulin dose and $\mathrm{HbA1c}$.

\begin{tabular}{|c|c|c|c|c|c|}
\hline & $\mathrm{N}(\%)$ & $\begin{array}{l}\text { Median } \\
\text { SMBG fre- } \\
\text { quency (/d) }\end{array}$ & $\begin{array}{c}\text { Mean HbA1c } \\
\pm S D(\%) \\
(\mathrm{mmol} / \mathrm{mol})\end{array}$ & $\begin{array}{c}\text { Spearman } \\
\text { correlation } \\
\text { coefficient }\end{array}$ & $P$ \\
\hline \multicolumn{6}{|c|}{ Stratified by education level } \\
\hline \multicolumn{2}{|c|}{ College and higher $91(44.2 \%)$} & 1.00 & $7.7 \pm 1.9(61)$ & -0.347 & 0.001 \\
\hline Middle school & $98(47.6 \%)$ & 0.93 & $8.7 \pm 2.7(72)$ & -0.245 & 0.025 \\
\hline Primary and lower & $17(8.3 \%)$ & 0.29 & $11.4 \pm 3.0(101)$ & -0.349 & 0.222 \\
\hline \multicolumn{6}{|c|}{ Stratified by insulin regimen } \\
\hline CSII & $39(19.5 \%)$ & 2.00 & $7.8 \pm 1.7(62)$ & -0.255 & 0.117 \\
\hline MDI & $93(46.5 \%)$ & 0.71 & $8.5 \pm 2.5(69)$ & -0.425 & $<0.001$ \\
\hline Traditional & $68(34.0 \%)$ & 0.42 & $9.2 \pm 3.0(77)$ & -0.233 & 0.081 \\
\hline \multicolumn{6}{|c|}{ Stratified by diabetes duration } \\
\hline$<1$ year & $55(26.2 \%)$ & 1.43 & $9.4 \pm 3.7(79)$ & -0.321 & 0.135 \\
\hline $1-5$ years & $58(29.7 \%)$ & 0.43 & $8.8 \pm 2.6(73)$ & -0.324 & 0.008 \\
\hline$>5$ years & $82(39 \%)$ & 1.00 & $8.0 \pm 1.9(64)$ & -0.356 & 0.001 \\
\hline \multicolumn{6}{|c|}{ Stratified by insulin dose } \\
\hline$<0.5 \mathrm{IU} / \mathrm{kg}$ & $39(21.2 \%)$ & 0.57 & $8.8 \pm 2.7(73)$ & -0.356 & 0.042 \\
\hline $0.5-0.8 \mathrm{IU} / \mathrm{kg}$ & $105(57.1 \%)$ & 1.00 & $8.2 \pm 2.2(66)$ & -0.315 & 0.001 \\
\hline$>0.8 \mathrm{IU} / \mathrm{kg}$ & $40(21.7 \%)$ & 0.46 & $9.3 \pm 3.3(78)$ & -0.381 & 0.018 \\
\hline \multicolumn{6}{|l|}{ Stratified by HbA1c } \\
\hline$<7 \%$ & $70(33.3 \%)$ & 2.00 & $6.3 \pm 0.4(45)$ & 0.071 & 0.602 \\
\hline$>=7 \%$ & $140(66.7 \%)$ & 0.57 & $9.6 \pm 2.4(81)$ & -0.313 & $<0.001$ \\
\hline
\end{tabular}

Correlations with statistical significance $(P<0.05)$ are shown in bold type. BMI-body mass index, CSII-continuous subcutaneous insulin infusion, MDImultiple daily injection.
693-P

Impact of an Educational Intervention on Foot Care among LowLiteracy and Unemployed Diabetic Patients

SUSANA CAROLINA BERTOLA, MARIA EUGENIA LOZANO, FABIANA MOLINA, PAULA BALDERRAMO GIL, JORGE WAITMAN, Córdoba, Argentina

Low-Educated and Unemployed (UE) patients are susceptible of developing foot lesions. A prospective longitudinal observational study designed to determine the impact of education on DM and foot care, metabolic control, number of ulcers, healing and amputations, adherence to scheduled appointments and comparing results between patients with low (LLL) and high (HLL) literacy levels and between the employed and the UE within the framework of a Public Hospital. Patients recruited: 291. Men: 51\%; Mean age: 54 years. LLL (up to primary school completion): $46 \%$. UE: 49\%. A first Survey 1 (S1) on baseline knowledge was conducted. Educational intervention interactive lecture on DM and foot care and foot examination followed by risk stratification. Assessment of acquired skills through Survey 2 (S2) Follow-up: 1 year. Significant $p<.05$. The overall survey scores improved significantly in reference to both LLL $(p=.014)$ and $\mathrm{HLL}(p=.001)$ patients. S and S2 scores were lower for LLL ( $p=.001$ and $p<.0001$ respectively). The study also showed an improvement among the working population ( $p=.003$ which was not evidenced among the UE. LLL and UE presented higher ulce and prior amputation rates against the HLL and employed and after the intervention the number dropped for new ulcers as well as for amputations but without statistical significance. Ulcers healed reached $58 \%$. Baseline A1C fell during follow-up among LLL $(9.39 \pm 2.34$ vs $7.83 \pm 1.53 \%$; $p=.0001)$ and UE $(8.91 \pm 2.45$ vs $7.96 \pm 1.85 \% ; p=.0126)$. Patients attending scheduled appointments amounted to $34.36 \%$. Education improved significantly the level of knowledge and skills as well as metabolic control among all participants. LLL patients and the UE presented a larger number of foot lesions. The rate of ulcer healing was high showing a trend of decreasing number of ulcers and amputations. Adherence to scheduled appointments proved low; hence the need to regard education as a continuous process, especially in reference to vulnerable populations.

694-P

Diabetes Risk Factor \& Prevention through Proactive CRM Counseling, its Effects on Prediabetes Patients

YOOMI BAE, SUNHEE KIM, SUNHEE AHN, YUMI HA, SUNGAH MIN, MINJUNG LIM, KYUMIN LEE, SUNGWOO PARK, KIWON OH, WONYOUNG LEE, CHEOLYOUNG PARK, EUNJUNG RHEE, SEEUN PARK, Seoul, Republic of Korea

Aims: The purpose of this study is designed to investigate the impact of Examinee CRM through counseling for diabetes-related factors over a period of one year, to determine the overall well being of the patients specializing in diabetes impaired fasting glucose.

${ }^{*}$ CRM (Customer Relationship Management): a professional medical consultation service tailored specifically for the patients well being and satisfaction.

Methods: 60 patients were screened and contacted through mail after a health examination. Their requirements were to have a fasting glucose level(FBS): $100 \sim 125 \mathrm{mg} / \mathrm{dL}$ and a body mass index(BMI) of $23 \%$ or more. Through phone calls, 30 patients from the experimental group were contacted by the diabetic nurse specialist, who would provide professional CRM Manual Based counseling on the risks, complications of diabetes (FBS, T-chol, TG, HDL-chol, LDL-chol, HbA1c, BMI), the importance of early detection, and lifestyle modifications. The remaining 30 patients did not receive professional CRM counseling, but just current phone call protocols. This comparative study was done over a period of one year.

Result: A comprehensive physical examination of the experimental and control groups after one year showed a difference in the results among the 7 items being tested, three of the inspection items/FBS: $109.0 \pm 6.0$ vs $97.5 \pm 8.6 \mathrm{mg} / \mathrm{dL}$, HbA1c; $5.6 \pm 0.2$ vs $5.4 \pm 0.3 \%$, BMl; $25.8 \pm 2.0$ vs $25.0 \pm 2.2 \mathrm{~kg} / \mathrm{m}^{2}$ )in the experimental group showed positive significant differences $(p<.001)$

Conclusion: Prediabetes patients, through proactive CRM mobile counseling to lower the FBS, HbA1c, BMI, were significant. After identifying the risk factor of these patients with prediabetes, CRM professional counseling emphasizes prevention and also an individualized plan for aggressive glucose management which will enable many years of a healthier quality of life. Counseling is very important in the prevention of diabetes and the many complications that may arise. 


\section{DIABETES EDUCATION} Diabetes Educators and Nurse Care Managers: Building Confidence for Ongoing Diabetes Support

JODI KRALL, PATRICIA JOHNSON, MARGARET THEARLE, LINDA M. SIMINERIO, Pittsburgh, PA

Patient Centered Medical Homes (PCMHs), organized around team care, are gaining attention to improve diabetes outcomes in primary care. Diabetes Self-Management Support (DSMS) is considered to be important in sustaining improved outcomes. PCMH nurse care managers (NCMs) are well-positioned to provide DSMS in primary care, but express concern in understanding the complexity of diabetes care. In recognition, a healthcare insurer employed 2 certified diabetes educators (CDEs) to train and support an extensive network of NCMs who provide disease management services to its members in PCMHs. CDEs administered a needs assessment to NCMs; responses were used to develop an ongoing diabetes training and support program. The program was designed initially to build NCMs' diabetes care knowledge base and subsequently evidence-based counseling strategies for DSMS. As a first step in measuring program impact, change in NCMs' confidence in diabetes care prior to and 6 months after program initiation was assessed. NCMs $(n=30)$ rated their diabetes confidence levels for 5 pre/ post items (5-point Likert scales). Perceived confidence improved in all areas: knowledge and skill level regarding diabetes medication and administration devices (pre 2.87, post $3.90 ;+1.03$ ); blood glucose monitoring skills (pre 3.86, post $4.50 ;+0.63)$; knowledge regarding signs, symptoms and treatment of hypo- and hyperglycemia (pre 4.10 , post $4.63 ;+0.53$ ); knowledge about types of diabetes (pre 3.77 , post $4.43 ;+0.66$ ); overall ability to assist patients living with diabetes (pre 3.47 , post $4.30 ;+0.83$ ). Building confidence in the area of diabetes care is fundamental to diabetes management and enhances the likelihood that NCMs will translate knowledge into practice. Using educators to train and support NCMs offers an opportunity to provide ongoing DSMS in the PCMH. Educators are in a unique position to use their expertise and skills at a broader level in order to assure the provision of quality DSMS

696-P

Evaluation of a Novel Educational Program for Healthcare Providers to Learn about the Empowerment: Diabetes Theater, a Workshop including Drama and Discussion

KENTARO OKAZAKI, HIROSHI OKADA, KEN OHASHI, TOSHIKAZU YAMAMOTO, TAKAYUKI ASAHINA, Kyoto, Japan, Tokyo, Japan, Okinawa, Japan

When we try to learn about the philosophy of the empowerment, one of the key elements might be how to build a good relationship between a patient and a healthcare provider (HCP). However, it is often difficult to learn this issue by only a one-way lecture-based program. Thus, we developed a novel interactive educational program, named Diabetes Theater (DT). This program was designed for audience to reflect on their own practice by watching the play based on common problems of misunderstanding between a patient and a HCP in a clinical practice, and joining the discussion by focusing the good points and improvements of the practice in the drama. The aim of this study was to make an evaluation of DT. Participants of DT were requested to answer a questionnaire at the 52nd annual scientific meeting of Japan Diabetes Society. A total number of respondents were 447 (male 85, female 362 ); dietitians $32 \%$; nurses $30 \%$; doctors $15 \%$; pharmacists $10 \%$; and others $13 \%$. About half of the respondents were certified diabetes educator of Japan. Both attitude and satisfaction were measured using Likert scales with $1=$ strongly disagree and $5=$ strongly agree. In the attitudes toward diabetes care, HCPs should: "be trained how to communicate with patients" (mean 4.5); "learn the skill of counseling for patients" (4.6); "learn how to set the goal with the patient" (4.6). In the level of satisfaction with the program, "A style of including a drama was interesting" (4.6); "The content of the drama was easy to understand" (4.5): "This program was helpful to improve my diabetes care" (4.4); "Overall I am satisfied with the program" (4.4); "I would like to join this program again" (4.4). In conclusion, DT was well accepted by HCPs as a unique program to earn the communication skill with patients for achieving the better patient-HCP relationship. This finding suggests that DT might be an interesting approach to improve the attitudes of HCPs in diabetes care in terms of the empowerment.

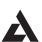

697-P

Insulin Treatment Safely Improving Glycemic Control in Muslim People With Type 2 Diabetes Mellitus During Ramadan Fast NAIM SHEHADEH, YASMIN MOAR, RAMADAN STUDY GROUP, Acco, Israel, Ramat Gan, Israel

Fasting during the Ramadan may have a harmful impact on muslin patients with T2DM. Our objective was to assess the efficacy and safety of Insulin detemir (Levemir) and Biphasic insulin (NovoMix70) during the Ramadan fast in an interventional, community based, comparative study. We conducted an open label, prospective, controlled multi-center cluster randomized study in Israel. In 6 randomly selected primary care clinics insulin treated T2DM patients received the intervention: Levemir and NovoMix70 protocol (Group $1, n=127)$ and in 6 others patients received standard care and treatment according to the American Diabetic Association recommendations (Group $2, n=120)$. In the intervention group, a new daily insulin dose was calculated as $60 \%$ of the usual daily insulin dose (DID). Levemir (40\% of the new DID) was given at sunrise (Suhur) and NovoMix70 (60\% of the new DID) was given before dinner (Eftar). DID was titrated. All patients performed daily 4 point glucose measurements (4PSMBG). The primary endpoint was to evaluate the difference in mean 4 PSMBG during days 23-30 of treatment between the two groups using a mixed effect model. Non inferiority was defined as a difference $<27 \mathrm{mg} \%$ on the full set. Mean age was 60 (SD 8.9) years in group 1 and 59 (SD 10.0) in group 2. Mean HbA1C was 8.4\% (SD 10.2) and mean weight was $87 \mathrm{~kg}$ (SD 16.1) in both groups. Duration of insulin treatment prior to study was significantly longer in group 1 ( 3.77 vs 2.75 years, $p=0.004$ ). There was no significant difference in the mean 4PSMBG during days 23-30 of treatment between the two groups ( $155 \mathrm{mg} \%$ vs 159 , respectively, $\mathrm{p}=0.12$ ). Non inferiority remained after controlling for length of insulin treatment. Hypoglycemia and syncope were significantly more common in the control group ( $20 \%$ vs $4.7 \%, p=0.0002$ and $3.4 \%$ vs $0, p=0.036$, respectively). We conclude that the combination of Insulin detemir (Levemir) and Biphasic insulin (NovoMix70) is non-inferior to standard care but is associated with less adverse events.

Supported by: Novo Nordisk, Inc.

698-P

Superiority of a Telemedicine Based Counselling in Ensuring Multi Drug Compliance in T2D

JOTHYDEV KESAVADEV, ARUN SHANKAR, GOPIKAKRISHNAN GOPALAKRISHNAN, JAYASREE LALLY, GEETHU SANAL, SUNITHA JOTHYDEV, Trivandrum, India

Non-adherence to polypharmacy is possibly most common reason for poor outcomes among T2D patients on treatment. We evaluated role of telemedicine (TM group) based follow up ensuring drug compliance \& better health outcomes compared to conventional treatment setting with occasional group education. Diabetes Tele Management System (DTMS ${ }^{\circledR}$ ) involves unique software \& trained multidisciplinary diabetes team communicating via phone/email for slow, steady titration of drugs \& tele-counselling.

Self-reported compliance questionnaire \& semi-structured interview were conducted in T2D enrolled in DTMS \& in general diabetes camps by Diabscreen Kerala (DK group) from Aug 2011-July 12. Respondents (83\%) included 2439 patients in TM of $>2$ follow up/ month with DTMS ${ }^{\circledR}$ \& 1985 patients in DK (Fig.1). On analysis, only $12.7 \%$ in DK were on insulin despite being suggested vs $72.4 \%$ in TM. DK group stopped all drugs( $12.7 \%$ vs $0.6 \%$ ), BP drugs $(47.6 \%$ vs $2.6 \%)$ \& statins $(74.8 \%$ vs $1 \%)$ more frequently than TM group due to various misconceptions. Drug compliance in TM is ensured via DTMS ${ }^{\circledR}$ by way of customized brief education modules, mitigating misconceptions about side effects of drugs with specific reference to continuation of statins, BP, anti-diabetes medications \& verifying purchase from hospital pharmacy. DTMS ${ }^{\circledR}$ ensured statistically significant drug compliance (93\%) vs conventional treatment setting (59.7\%) underscoring utility of telemedicine as a cost effective patient education tool.

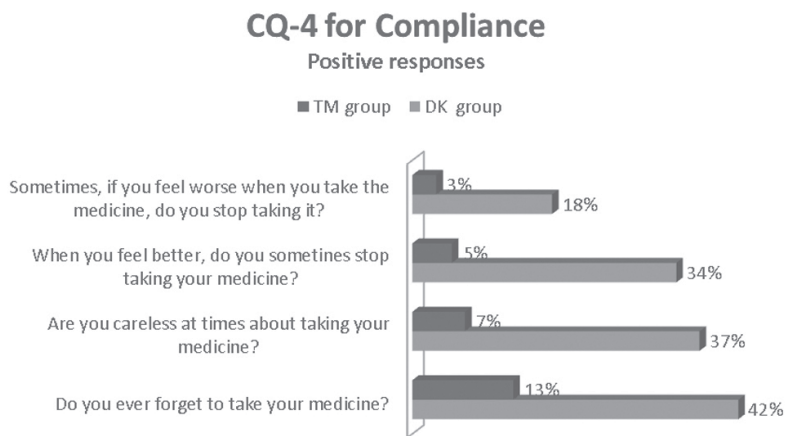


699-P

Using an Educational Intervention to Improve Inpatient Glycemic Control One Intern at a Time

JANE JEFFRIE SELEY, DAVID J. BRILLON, YA-LIN CHIU, LINDA M. GERBER, NAINA SINHA, New York, NY

Background: Despite a growing body of evidence that improved glycemic control in hospitalized patients is beneficial, many patients do not achieve targets. Strategies have been implemented to streamline insulin dosing, making it easier for prescribers to safely and effectively initiate and intensify subcutaneous insulin. In 2010, we implemented a comprehensive insulin order set with minimal housestaff education. In 2011, we evaluated the efficacy of our intervention and learned that the rate of hypoglycemia for medical and surgical non-ICU patients was significantly reduced $(p<.0001)$ yet the percentage of patients in target ranges decreased and hyperglycemia rates increased significantly $(p<.0001, p<.0001$ respectively). Primary Aim: To implement and evaluate a problem based learning (PBL) intervention to teach medicine PGY-1 housestaff how to fully utilize the insulin orderset, focusing on when and how to titrate doses.

Methods: Each week in 2012, a new medicine intern met with the diabetes nurse practitioner/CDE for a one hour educational intervention with computer-based step-by-step instructions on how to optimally utilize the order set using case-based examples. Their new knowledge was applied by rounding with the inpatient diabetes consult team for the next four days. Blood glucose $(B G)$ testing reports were analyzed to determine rates of hypo- and hyperglycemia and target BGs on medicine units.

Results: Hyperglycemia was significantly decreased from baseline in 2010 (39.1\%) compared to post-intervention in 2012 (35.55\%, p<.0001). Although the hypoglycemia rate continues to be a small number of patients, it did increase from $2.03 \%$ to $2.49 \%(p=0.0014)$.

Conclusions: An educational intervention by the diabetes educator was effective in significantly lowering rates of hyperglycemia and increasing the number of patients in the target range of $70-179 \mathrm{mg} / \mathrm{dl}$. Further education is needed to address increased rates of hypoglycemia without a subsequent rise in hyperglycemia.

700-P

Components of Glu, An Online Social Network for Engaging Individuals With Type 1 Diabetes in Research

CHRISTINA L. ROTH, GAYATHRI SRINIVASAN, Boston, MA

We describe innovative features of a patient centric social network, Glu, which is exclusively designed for engaging individuals with T1D and caregivers in peer education and research. Glu is part of the T1D Exchange which includes a clinic network, a clinic registry of $26 \mathrm{~K}+\mathrm{T1D}$, and a biorepository.

Glu is a web \& mobile enabled platform, allowing individuals with T1D ages 13 and older and/or their caregivers to join. Members create a profile with customized privacy settings, form bonds with other members, and participate in discussions. GluU is a part of Glu which allows members to participate in surveys/studies via web or mobile. A central IRB governs the participation of individuals, who first sign an informed consent (individuals $\geq 18 \mathrm{yrs}$, parent/guardian of child $<18 \mathrm{yrs}$, caregiver of individuals $\geq 18 \mathrm{yrs}$ ) or assent (children 13-17 yrs), giving permission to collect information about demographics, medical history, lifestyle, feelings/issues about diabetes.

Glu was formally launched in November, 2012 and has 4200 members. The average age of T1D members is $35 \mathrm{yrs}$. Among those providing data, $70 \%$ use insulin pump and 30\% use injections; $32 \%$ are using a CGM.

Advantages of Glu include the ability to leverage social network for identifying research participants, the opportunity to accelerate the time frame of studies, and the ability in the future to link clinical outcomes data from the registry to a Glu user profile. We face some challenges, including verification of T1D disease status, population bias of the online community limiting generalizability, and the ethical and logistical challenges of conducting clinical research outside of the traditional clinic model. Glu represents a new paradigm for conducting T1D research using the Web 2.0 and mobile technologies.

Supported by: Leona M. and Harry B. Helmsley Charitable Trust
701-P

Electric Health Record (EHR)-Based Intensive Coaching Program "TOGANE" Prevents the Progression of Diabetic Nephropathy in Japanese

HARUMI NISHIHARA, SADAKO WAKAMATSU, HIROMI MAEDA, MIYUKI HANAZAWA, MICHIKO AOKI, FUJIKO MIYAHARA, TATSUO KATSUKI, TAKENORI SAKAI, YOSIHUMI TOYOJI, SHIGEKI IMAMURA, AIZAN HIRAI, Togane, Japan, Hunabashi, Japan, Asakusa, Japan, Komatsu, Japan, Yawata, Japan, Minano, Japan

The increase in diabetic patients and its complications requires intensive disease management of diabetes in Japan. We have constructed and operated regional EHR since 2011. Diabetic patients more than 5,000 have been registered to our EHR. This EHR system consists of two mapping systems for disease management. First is a personal mapping system named "Case management MAP", which is a tool for case management of individuals to achieve optimized therapy using minimum data set for diabetes. Second is a regional disease management mapping system named "Regional disease management MAP", which is a tool to triage diabetic patients having a high priority for treatment from whole diabetic patients based on abnormal values of major minimum data set. The present study was performed to evaluate the effectiveness of intensive coaching program "TOGANE (TeamOriented Generous Assist for the patients with diabetic NEphropathy)" for preventing the progression of diabetic nephropathy. Program "TOGANE" is mainly consist of the teaching tools and workflow by CDE and dietitian for the salt restriction in the treatment of diabetic nephropathy. In the present study, 600 diabetic patients were included. The patients were divided to two groups, conventional therapy group $(C)$ and conventional therapy plus TOGANE group(T). Stage 2, (C)169 vs (T)117, Stage 3, (C)144 vs (T)88, Stage 4, (C)47 vs(T) 32. Study period is 1 year. Renal function(eGFR, urinary albumin excretion, proteinuria) and several clinical parameter are determined at every two months. At the period of 8 months, microalbuminuria of the patients of group (C) in Stage 2 increased from $75.5 \mathrm{mg} / \mathrm{g}$ Cre to $122.3 \mathrm{mg} / \mathrm{g}$ Cre $(p<0.01)$, while that in group (T) in Stage 2 did not change $(60.9 \mathrm{mg} / \mathrm{g}$ Cre to $64.1 \mathrm{mg} / \mathrm{g}$ Cre). In Stage 4, the renal function in group (T) did not decreased, while that in group (C) decreased. Thus, program "TOGANE" can be optimized by the combination with EHR for preventing the progression of diabetic nephropathy.

Nonvisual Foot Inspection for People With Visual Impairment ANN S. WILLIAMS, BRYAN D. CALDWELL, JILL S. KAWALEC-CARROLL, Cleveland, $\mathrm{OH}$, Independence, $\mathrm{OH}$

People with diabetes and visual impairment have high risk for foot problems. A nonvisual method for inspecting their own feet, using the senses of touch and smell, may help them discover new foot problems while in early, easily-treated stages. This simple, low-cost technique may decrease incidence of foot ulcers and amputations.

The purpose of this pilot study was to determine the initial efficacy, feasibility, and acceptability of a technique for nonvisual foot inspection, using the hands to inspect the feet tactually, and the nose to detect unusual odors.

Thirty people with diabetes and visual impairment were recruited and assigned to experimental or comparison groups. Both groups received comprehensive diabetes self management education with emphasis on foot care and instructions to call the podiatrist if new foot problems were found. The experimental group was taught non-visual foot inspection, the comparison group to have a sighted person check the feet. All received bimonthly phone calls to document foot care practices at home. All had a baseline podiatry evaluation, with 4 follow-ups over a year, to document discovery of new foot problems at home and by the podiatrist. Focus groups were held immediately after each cohort had completed classes and one year later, transcribed and analyzed for themes.

Analysis included rates of home foot checks, probability of reporting new foot problems to the podiatrist, and total numbers of new foot problems reported. Learning nonvisual foot examination had a dramatic effect on rates of home foot checks. The experimental group reported checking their feet 5.45 times per week, while the comparison group reported 1.74 . They also had a higher probability of reporting new foot problems to the podiatrist, and more total foot problems reported. Qualitative analysis of the focus groups strongly reflect a preference for self-examination of feet over asking someone else to check the feet.

Initial efficacy, feasibility, and acceptability are high and support the need for study in a larger group.

Supported by: IDF 


\section{DIABETES EDUCATION}

Effect of Lifestyle Modification on Serum Chemerin Concentration and its Association With Insulin Sensitivity in Overweight and Obese Adults With Type 2 Diabetes

SO HUN KIM, SEUNG HWAN LEE, KI YONG AHN, DONG HOON LEE, YOUNG JU SUH, YUN JIN CHOI, SEONG BIN HONG, YONG SEONG KIM, JUSTIN Y. JEON, MOONSUK NAM, Incheon, Republic of Korea, Seoul, Republic of Korea

Chemerin is a recently identified adipokine which has been reported to be associated with adiposity, insulin resistance, risk factors of metabolic syndrome and inflammation. In this study, we evaluated whether there are significant changes in $\mathrm{HbA1c}$ and serum chemerin levels after a 12 week lifestyle intervention in overweight and obese adults with type 2 diabetes.

A total of 35 overweight or obese subjects with type 2 diabetes were randomized to receive intensive lifestyle modification including supervised exercise sessions or usual care for 12 weeks. Anthropometric and clinical data were collected before the intervention and after 12 weeks.

There was a significant decrease in $\mathrm{HbA} 1 \mathrm{c}(-1.0 \pm 0.5$ vs $0.1 \pm 0.6 \%, \mathrm{p}<0.001)$, BMI (-1.3 \pm 0.9 vs $-0.1 \pm 0.7 \mathrm{~kg} / \mathrm{m} 2, p<0.001)$ and total body fat content (fat percent change $-3.7 \pm 2.7$ vs $-0.4 \pm 1.8 \%, p<0.001)$ and a significant increase in V02 $\mathrm{max}(+3.7 \pm 3.9$ vs. $-1.0 \pm 2.7 \mathrm{ml} / \mathrm{min} / \mathrm{kg}, p<0.001)$ in the intervention group compared to the usual care group after 12 weeks. Serum chemerin level was significantly decreased in the intervention group compared to the usual care group $(-8.1 \pm 21.6 \mathrm{vs} .+8.2 \pm 15.9 \mathrm{ng} / \mathrm{ml}, \mathrm{p}=0.021)$. Baseline chemerin levels were positively correlated with HOMA-IR $(r=0.36, p=0.04)$ and hSCRP $(l=0.37, p=0.049)$. Change in chemerin concentration during 12 weeks was independently negatively correlated with change in insulin sensitivity index $(\beta=-0.39, p=0.01)$ and positively correlated with change in fasting plasma glucose $(\beta=0.32, p=0.029)$, total cholesterol $(\beta=0.26, p=0.038)$, and lipocalin-2 ( $\beta=0.33, p=0.008)$.

In conclusion, an intensive lifestyle intervention including supervised exercise was able to decrease serum chemerin level significantly. Decrease in serum chemerin level was associated with improved insulin sensitivity and this may be involved in the beneficial effects of lifestyle intervention in overweight and obese type 2 diabetic patients.

704-P

Evaluation of Self-Management, Self-Care Ability and Medication Adherence In OPENING (Organization Program of DiabEtes INsulIN ManaGement) Study

LINONG JI, JIE LIU, JUMING LU, XIAOHUI GUO, Beijing, China, Taiyuan, China

Purpose: Organization Program of DiabEtes INsullN ManaGement (OPENING) study, was designed to evaluate the self-management, selfcare ability and medication adherence in T2DM patients managed with structured education

Methods: Eligible patients were over 18 years of age and inadequately responded to two or more OADs for over 3 months ( $\mathrm{HbA1c} \%>7.5 \%$ ). Patients were randomized (1:1) into education group (structured education plus insulin therapy) and control group (usual care plus insulin therapy). Structured education was conducted by trained nurses with OPENING education tool, which included seven models as medications, insulin injection technique, BG monitoring, diet, physical activity, hypoglycemia prevention and complication prevention. Chinese version Diabetes Management Selfefficacy Scale (C-DMSES), Summary of Diabetes Self-Care Activities Scale (SDSCA), and Morisky Medication Adherence Scale (MMAS) were used to assess patients' ability of self-management, self-care and medication adherence, respectively.

Results: Totally 1511 patients with T2DM were enrolled from 48 hospitals across China. Of the 1289 patients who completed this study, 646 were in education group and 643 in control group. After 16-week, the total score of C-DMSES increased in both group, and significantly higher in education group(13.87 \pm 31.00 vs. $20.27 \pm 33.17, P<0.0001)$. The total score of SDSCA increased in both group, and significantly greater in education group $(10.50 \pm 16.07$ vs. $6.97 \pm 14.63, P<0.0001)$. Propotion of patients with high adherence in MMAS has increased in both groups $(\mathrm{P}<0.0001 \mathrm{vs}$. baseline). And education group had a higher proportion of high compliance $(74.53 \%$ vs. $62.99 \%, P<0.05)$.

Conclusions: Structured education can improve the ability of selfmanagement and self-care in patients with type 2 diabetes, meanwhile enhance their medication adherence. Basal Insulin and Beyond in the U.S.: Real World Attainment of Glycemic Goals

MAUREEN J. LAGE, BRADLEY H. CURTIS, KRISTINA S. BOYE, Delray Beach, FL, Indianapolis, IN

This study examined the use of basal insulin and common next steps in therapy, in relation to achievement of glycemic control (A1c $\leq 7 \%)$ among patients with type 2 diabetes (T2D).

Medical records and claims, and prescription claims from a sample of 565,120 patients with T2D were obtained (Humedica Data). Inclusion required T2D diagnosis; first prescription of basal insulin July 1, 2008 to March 30, 2009, data available for 1yr prior and 2yrs post index date (1st prescription), and $A 1 c$ values in the pre and post period. Descriptive statistics compared achievement of glycemic control post index. Logistic regression examined associations between insulin regimen and the probability of achieving target A1c controlled for patient characteristics, diabetes medication, pre-index A1c, and post index frequency of A1c testing. A time to glycemic control analysis was performed using the Kaplan-Meier estimator and the log-rank test.

4613 patients (51-64yrs 35.9\%; >64yrs 52.1\%; BMI 35.5; female 51.6\%) were identified; $59 \%$ of patients achieved target $\mathrm{A} 1 \mathrm{c} \leq 7 \%$ at any stage during the $2 y r$ post period. Multivariate logistic regression indicated that compared to patients who continue with basal insulin (C) patients who augment (A) with additional insulin (OR $=0.495 ; 95 \% \mathrm{Cl} 0.419-0.583)$, switch $(\mathrm{S})$ to an alternative type of insulin (OR=0.388; $95 \% \mathrm{Cl} 0.288-0.523$ ) or discontinue (D) insulin therapy (OR=0.259; 95\% $\mathrm{Cl} 0.0177-0.379$ ) were significantly less likely to achieve target A1c. Indeed, 24-mths post first basal prescription, $66 \%(C), 49 \%(A), 37 \%(S)$, and $13 \%$ (D) of patients had achieved A1c $5 \%$ (logrank test: $p<0.0001)$.

A high proportion of patients with $\mathrm{T} 2 \mathrm{D}$, treated with basal insulin alone, are not achieving recommended target A1c, and it appears that commonly utilized treatment approaches beyond basal therapy may be ineffective. Further prospective research is required to better characterize those patients who may benefit from basal only therapy vs. alternative approaches such as pre-mix, basal bolus therapy, or GLP agents.

Supported by: Eli Lilly and Company

706-P

Recruiting for a Text Message Intervention for Rural African-American Women With Diabetes

NATALIE B. MAY, CHRISTOPHER WINSTEAD-DERLEGA, PAUL ZIMMERMAN, MARGARET PLEWS-OGAN, MARGARET NEES, REBECCA DILLINGHAM, MOHAN NADKARNI, ROBERT GREEN, Charlottesville, VA

Cell phone text messaging has shown promise in improving self-care among some populations of diabetics. Its relative low cost and potential to provide benefits of ongoing self-management support warrants exploration of its feasibility among diverse patient groups. We developed a diabetes selfcare program, Call2Health (C2H), to help socioeconomically disadvantaged African-American women living in a small city and surrounding rural areas. Our study compares the effectiveness of two interventions compared to usual care: (1) daily personalized text message reminders and affirmations to support each woman's health care behavior goals and (2) $\mathrm{C} 2 \mathrm{H}$ group visits where women learn about diabetes self-management and gain social support and encouragement. To enroll in the study, women had to have access to their own cell phone with text messaging capability and be able to attend group sessions if they were randomized into that study arm. We identified eligible patients in several ways, including a daily clinic database review for women to approach during clinic visits. A study researcher met with each patient, explained the study, and invited them to participate. We assessed 330 patients for eligibility in the $\mathrm{C} 2 \mathrm{H}$ study and enrolled 48 of them. Of the remaining 282 eligible women, 50 lived too far away and would be unable to participate in the group session if randomized there; 19 were too ill to participate. Over $36 \%$ could not participate due to lack of text message capability: $72(26 \%)$ had no cell phone; $27(10 \%)$ had cell phones with no texting capability; and $4(<2 \%)$ did not want to pay for extra texts. Of the remaining 110 eligibile women, 67 (29\%) declined to participate; 43 women said they were "interested" but did not enroll. There are many barriers to receiving high technology care in disadvantaged patient populations. Although promising technology, researchers may overestimate cell phone usage amongst the population and thus may need to provide cellphone and text messaging service to study participants.

Supported by: Bristol-Myers Squibb 
707-P Cities for Life: Gaps in Perception between Primary Care Providers and Patients Regarding Diabetes Management

ADAM G. TSAI, EDWIN B. FISHER, NATALIA LOSKUTOVA, SHARON HUNT, T. MICHAEL HARRINGTON, TAMELA J. TURNER, WILSON D. PACE, Aurora, CO, Chapel Hill, NC, Leawood, KS, Birmingham, AL

Patients and primary care providers (PCPs) may have unique views about what is needed to support disease self-management. This may be especially true of diabetes, which typically involves 3 to 4 PCP visits per year, but for the patient is a "24-hour disease." To test this view, we conducted random digit-dial telephone surveys of PCPs $(n=50)$, patients $(n=201)$ and community members ( $\mathrm{n}=309$ ) in Birmingham, Alabama as part of "Cities for Life," an ongoing, clinical-community diabetes management program led by the American Academy of Family Physicians Foundation. Overall, participants identified diabetes as a major health priority. Most patients and community members felt that supporting community programs to manage diabetes is a good investment. However, fewer patients, as opposed to community members, felt that existing support was adequate and only a minority of both groups stated they used these programs. PCPs believe that their patients do not have adequate family support and that patient education is the greatest need. However, a majority of patients stated that their family support and knowledge to manage their disease are adequate. Over $1 / 3$ of patients reported a lack of motivation as a barrier to diabetes management, and over $60 \%$ of patients stated that they were already regulating their diabetes. PCPs recommend medication more frequently than community programs for lifestyle modification. PCPs stated that structured weightloss and exercise programs are most helpful, but patients stated that parks and healthy food options are most helpful. In summary, PCPs, patients and community members often do not share the same views regarding diabetes management. Patients often struggle with motivation for behavior change. Explicit motivational language from PCPs about the benefits of lifestyle modification and medications accompanied by other strategies such as reminders for patients to engage in community programs may help to bridge communication gap.

Supported by: Sanofi U.S.

708-P

Development of a Young Adult Type 1 Diabetes Knowledge Assessment Tool

JONI K. BECK, STEVE A. STERNLOF, CYNTHIA A. MUHAMEDAGIC, CHRISTINA M. SHAY, KAI DING, EVAREN E. PAGE, JAMES T. LANE, Oklahoma City, OK

The purpose of this study was to develop a diabetes knowledge assessment tool to use in young adults with type 1 diabetes (T1D) and their caregivers and examine associations between assessment score with demographics and hemoglobin A1c (A1C). Participants with T1D ages 1522 years were included. Two 25 item, multiple choice questionnaires were developed; one for assessment of patient knowledge, one for assessment of caregiver knowledge. Questions were focused on the 9 core curriculum topics as defined in the National Standards of Diabetes Self-Management Education (American Diabetes Association), including disease process, nutrition, exercise, medications, monitoring, acute/chronic complications, psychosocial issues and behavior change. Assessment scores for both groups were defined as the number of correct items. Multiple linear regression was used to examine associations between participant and caregiver scores with A1C and demographic characteristics. Twenty-two participants with T1D were enrolled (mean age $16.9 \pm 2.0$ (mean \pm SD), diabetes duration $8.1 \pm 4.7$ years, $50 \%$ male, $68 \%$ Caucasian, 15 on multiple daily injections (MDI); 7 on continuous subcutaneous insulin infusion (CSII)) and 14 caregivers completed the questionnaire. Assessment scores were similar among groups (20.9 vs. 21.1, $p=0.69$, respectively) and between patients on MDI or CSII (20.9 vs. 20.9, $p=0.95$, respectively). Higher caregiver score - but not participant scores - were associated with lower A1C after accounting for age, diabetes duration and sex $(\mathrm{p}=0.03)$. After similar adjustment, mean caregiver score was higher in the private-insured group vs. the Medicaid group (score difference $=2.4, p=0.04$ ). This diabetes knowledge assessment can be used to identify T1D patients requiring additional education interventions, assisting in a successful transition to adult care and correlates with A1C level and payer source. Further use of the questionnaire is needed to demonstrate improvements in acquiring diabetes knowledge.
709-P

Implications of ACCORD Trial Are Unclear to Clinicians Managing Patients With T2DM

MARGERY J. TAMAS, ANGELA C. MCINTOSH, AMARYNTHIA CARBONARA, STEVEN A. WEINMAN, Atlanta, GA, Somerset, NJ

ACCORD demonstrated that some individuals with T2DM may be harmed by intensive glycemic goals $(\mathrm{A} 1 \mathrm{C}<6 \%$ ) and that there is a complex relationship between hypoglycemia and mortality. These results and similar findings in other large trials led to current ADA guidelines emphasizing individualized glycemic goals, and particularly to relax goals in older patients and individuals with comorbidities. We hypothesized that clinicians may misinterpret ACCORD and will have difficulty applying the new ADA guidelines in practice.

Registrants for regional meetings on insulin therapy were asked to state the influence of ACCORD on the management of T2DM. Responses were obtained on 109 of 322 registrations. Textual analysis was performed, stratified by city and degree status. Responses were coded for concordance with risk-based individualization of therapy and $\chi^{2}$ applied to the categorical data.

Responses indicated widely divergent interpretations of ACCORD, without statistically significant differences by city $(P=.457)$ or by degree status $(P=$ $.766)$. Of concern, $9.2 \%$ reported they were unfamiliar with ACCORD and $22.9 \%$ reported inappropriate practice changes. Several clinicians confused ACCORD with NICE-SUGAR, an inpatient study which also found increased mortality with tighter control.

ACCORD is often misinterpreted by clinicians managing patients with T2DM. Education on appropriate risk-based individualization of therapy is needed to implement new ADA guidelines.

\begin{tabular}{lccccccc}
\hline Response & $\begin{array}{c}\text { Atlanta, } \\
n(\%)\end{array}$ & $\begin{array}{c}\text { Dallas, } \\
n(\%)\end{array}$ & $\begin{array}{c}\text { Phoenix, } \\
n(\%)\end{array}$ & $\begin{array}{c}\text { MD/DO NP/PA/PhD/MS/ } \\
\text { MSN/PharmD }\end{array}$ & $\begin{array}{c}\text { Other } \\
\text { Degree }\end{array}$ & $\begin{array}{c}\text { Total, } \\
\text { ( }(\%)\end{array}$ \\
\hline Concordant & $11(31.4)$ & $9(22.5)$ & $8(23.5)$ & $6(20.7)$ & $13(27.1)$ & $9(28.1)$ & $28(25.7)$ \\
\hline Neutral/ambiguous & $19(54.2)$ & $22(55.0)$ & $15(44.1)$ & $18(62.1)$ & $23(47.9)$ & $15(46.9)$ & $56(51.3)$ \\
\hline Discordant & $5(14.2)$ & $9(22.5)$ & $11(32.3)$ & $5(17.2)$ & $12(25.0)$ & $8(25.0)$ & $25(22.9)$ \\
\hline Total & $35(100)$ & $40(100)$ & $34(100)$ & $29(100)$ & $48(100)$ & $32(100)$ & $109(100)$ \\
\hline
\end{tabular}

Supported by: Novo Nordisk, Inc.

710-P

The Effect of the Packed Sample Meal Experience on the Glycemic Control in Korean Type 2 DM Patients

JIN SUN CHOI, EUN MI KIM, HEE YOUNG KIM, SE-EUN PARK, CHUL YOUNG PARK, SUNG WOO PARK, Seoul, Republic of Korea

Aims: Various practical tools are applied to enhance the effect of nutrition education of diabetes. Provision of packed sample meal to diabetic patients may give a chance to understand their meal plan. We investigated the effect of packed sample meal experience on the glycemic control of Korean T2DM patients.

Methods: The subjects were 343 out-patients with T2DM treated at DM Center, Kangbuk Samsung Hospital for more than 24-months. Intervention group (PSM group) consisted of 152 patients who were provided packed sample meals (PSM) one more times. PSM subjects were given PSM based on their energy requirements after FBS test and postprandial plasma glucose test was carried out. We compared the status of glycemic control of PSM group and control group at $12 \& 24$-month.

Results: At base line, there was no significant difference between PSM group \& control group except age (PSM 58.6, control 61.3 years old, p 0.031). Mean number of PSM experience was 7.6 ( $\min 1, \max 16)$ for 2 years. In PSM group, the levels of FBS, pp2hr plasma glucose \& $\mathrm{HbA1c}$ were significantly improved at 12- \& 24-months. But HbA1c level significantly increased at 24 -months in control group (baseline $6.83 \%, 24$-months $7.01 \%, p<0.001$ ). The percentage of improvement in FBS (PSM 2.4\%, control $-3.8 \%, p=0.04$ ), pp $2 \mathrm{hr}$ (PSM 18.4\%, control $-10.1 \%$, p $<0.001$ ) and HbA1c (PSM 6.1\%, control $-3.5 \%$, $p<0.001$ ) were greater in PSM group compared control group at 24-months after adjustment for age. There was positive correlation between the number of PSM experience and the percentage of $\mathrm{HbA1c}$ improvement at 24-months after adjustment for age \& baseline HbA1c level $(r=0.26, p=0.001)$.

Conclusion: Our study provides some evidence that packed sample meal experience may helpful to glycemic control of T2DM patients. Provision of packed sample meal may be a useful nutrition education tool for selfmanagement 


\section{DIABETES EDUCATION}

Evidence Based Physicians' Training Program Assists Achievement of Treatment Targets and Improves Standards of Care: Results From the IMPROVE Control Program

ASHOK KUMAR DAS, SUBHASH KUMAR WANGNOO, AJAY KUMAR, SANJAY KALRA, A.G. UNNIKRISHNAN, SHAHID AKHTAR, RAMAN V. SHETTY, Pondicherry, India, New Delhi, India, Patna, India, Karnal, India, Kochi, India, Bangalore, India

The IMPROVE Control Program hypothesized that a structured, evidence based physicians training program would assist their patients achieve treatment targets and improve standards of diabetes care in India. To evaluate its impact, a multi-centre, non-intervention study was conducted. It included patients with type 2 diabetes (T2D) who had an A1C $>9$ at time of diagnosis or an $\mathrm{HbA1C}>7 \%$ even after six months of initiation of therapy with antidiabetic agents. The study evaluated 959 physicians, of whom 717 had undergone the structured training (trained group, TG) and 242 physicians formed a control, untrained group (UG). Data from 21367 patients with T2D (TG, UG = 16476 and 4891, respectively) was collected at baseline, week 13 and week 26. Primary endpoint was proportion of patients achieving a glycaemic target of $\mathrm{A} 1 \mathrm{C}<7 \%$ in 180 days. Other endpoints included mean change in A1C from baseline and evaluation of the physicians' perceptions. Poor glycaemic control [Mean (SD): A1C $=9.4$ (1.3), FPG (mg/ dl) $=181.2$ (45.7); and PPG 263.6 (68.5)] at baseline highlighted need for optimal insulinbased therapy. At baseline, 6608 (32.2\%) reported history of existing complications of diabetes. At week 26 , in TG, $22.6 \%$ achieved the primary endpoint compared to $14.0 \%$ in UG $(p<0.001)$. The mean change in A1C was also better (-1.8 in TG vs. -1.6 in UG, $p<0.001)$. Besides, $71.5 \%$ of patients in TG recorded a total cholesterol of $<200 \mathrm{mg} / \mathrm{dl}$ compared to 64.9 $\%$ in untrained group $(p<0.001)$. At week 26 , episodes of hypoglycaemia were similar in both groups. Once daily premixed insulin subsequently intensified to twice daily was rated as the most optimal insulin regimen for initiation and intensification of therapy (44.8\%) followed by once daily basal insulin start intensified to twice daily premixed insulin (28.5\%). The authors conclude that the IMPROVE Control Program has made a positive impact on glycaemic control and standards of diabetes care in India.

712-P Explaining Patients' Responses to Diabetes Education: A Qualitative Study

ELIZABETH A. BEVERLY, MARILYN D. RITHOLZ, JONATHAN S. BISHOP, DANA L. BRUCK-SEGAL, KELLY M. BROOKS, NINA F. LEWIS-SCHROEDER, OM P. GANDA, SHANE FITZGERALD, ENRIQUE CABALLERO, MEDHA MUNSHI, KATIE WEINGER, Boston, MA

To explore factors associated with responding to an intervention, we interviewed 45 type 1 and type 2 diabetes adults (80\% White, 56\% female, $49 \%$ type 1 diabetes, aged $59 \pm 11,16 \pm 2$ yrs of education, $A 1 C=8.0 \pm 1.3 \%, 26 \pm 17$ yrs duration): 15 patients who maintained glycemic targets $\mathrm{A} 1 \mathrm{C}<7.0 \%$ for a minimum of 4 years (Long-term $<7.0 \%$ ) and 30 who participated in a diabetes education intervention 5 years prior (15 who improved A1C 3-months post intervention $>0.75 \%$, Improvers and 15 who did not improve, Nonimprovers). Interviews were transcribed, coded, and analyzed via content analysis using NVivo 8 software. Groups did not differ by age, gender, years of education, type of diabetes, or diabetes duration. Although 7 Improvers maintained improvements for 1 year, only 3 maintained improvements for 5 years, and 0 achieved glycemic targets at 5 years. Qualitative analysis revealed that individuals in the Long-Term $<7.0 \%$ A1C group described "knowing what to do and doing it" in their diabetes self-management. They emphasized the importance of following a self-care routine and valued organization and consistency. Further, these individuals adopted a proactive approach to learning about their diabetes over time and receiving frequent follow-up with their diabetes care team. Both Improvers and Nonimprovers reported knowing what to do for self-care and recognized the value of a routine; however, Nonimprovers felt unable to carry out these behaviors because following a routine "was not in their nature." Improvers initially were able to perform self-care but cited lack of discipline, laziness and aversion to routine as reasons for not maintaining improvements. These findings suggest that patients who are struggling with self-care and glycemia may benefit from highly structured programs with frequent reinforcement to help them develop and maintain new lifestyle behaviors that will last over a longer term.

Supported by: NIDDK (2R01DK60115-06)
Evaluation of a Carbohydrate Counting Course during a Clinic Setting in Management of DM1

MAEVE C. DURKAN, HANNAH FORDE, HILDA CLARKE, Galway, Ireland

DAFNE is a carbohydrate counting course used in patients with DM1. It runs over a week and delivery is difficult with staff constraints. Published results are disappointing with no evidence (Irish Dafne group) to show durable effect.

Our study aimed to (1) examine the feasibilility of delivering a ' 3 hour' carbohydrate counting' (CC) program in the setting of a diabetes clinic, (2) evaluate the use of CC in managing DM1 and (3) compare outcomes with a control group not using CC.

20 patients with DM1 were randomly selected and assigned to one of 2 groups ( $\mathrm{CC}$ or a dietary lecture). All had baseline HbA1c, lipids, weight, and eGFR recorded. All completed a Berger QOL questionnaire, were specifically asked about hypogylcemia and re-assessed after 24 weeks.

The control group attended a 2 hour lecture delivered by a dietician.

The CC group attended a 3 hour workshop, having brought their own 'foods' and delph ware, and were taught CC by the diabetes nurse.

The CC course was easily delivered in a clinic setting. It became evident, that while patients were motivated, they found it laborious measuring food portions, making calculations and continuous engagement was problematic.

At 24 weeks, HbA1C in the CC group increased, $p<0.01$. It remained unchanged in the control group $(p=0.944)$. Independent T-Test showed that the CC intervention was not more effective $(p=0.834)$.

There was a non- significant trend towards a reduction in hypoglycaemic events in the CC group $(p=0.51)$. There was no diference between both groups.

There was a non- significant difference in $00 \mathrm{~L}$ between both groups, with the $\mathrm{CC}$ group reporting less anxiety about hypoglycemia.

Interestingly, the CC group had poorer self reported Q0L, (24.79 Vs 13.78). This may have hampered their ability to engage with interventions.

In summary, It is feasible to deliver this program. Patient selection is important. Enthusiasm does not equal success, and ongoing motivation is a limitation. CC success may not lie in reducing $\mathrm{HbA1c}$, but in reducing hypoglycemia and improving $\mathrm{OOL}$.

\section{4-P}

Diabetes Care Barriers Increasing at a Large Municipal Hospital CATHERINE S. BARNES, JANE M. CAUDLE, MARY RHEE, JADA BUSSEY-JONES, CIRCE W. TSUI, DAWN SMILEY, CHRISTOPHER NEWTON, VISHAL PAREKH, NKECHI MBAEZUE, CINNAMON BRADLEY, JOLENE LOWERY, GUILLERMO E. UMPIERREZ, PAYAL SHAH, LAWRENCE S. PHILLIPS, JOYCE DOYLE, DAVID C. ZIEMER, Atlanta, GA

Despite improvements in medical education and the availability of new therapeutic agents, there is little understanding of barriers to diabetes care as perceived by physicians in training. Since such physicians are the primary care physicians of the future, we surveyed 112 internal medicine residents in the largest primary care clinic at Grady Hospital in Atlanta, a major municipal center, and compared results to a previous survey of 152 residents in 1999

Healthcare system barriers: In 2012 vs. 1999, 55\% vs 51\% reported problems with insufficient time during clinic visits $(p=N S)$, inadequate staffing for patient education ( $62 \%$ vs $45 \%, P=0.009$ ), and missing progress notes or lab data that made care difficult ( $82 \%$ vs $85 \%$, NS). Although $91 \%$ vs $93 \%(p=N S)$ saw current formulary medications as effective, many felt that newer incretin-based medications would help (34\% vs $26 \%$, $p=N S$ ).

Patient barriers: $78 \%$ vs $62 \%$ found literacy and health care costs to be problems $(p<0.01)$, but most felt that patients understood the importance of good control and took diabetes seriously. While $51 \%$ vs. $36 \%(P<0.02)$ felt that patients do follow treatment recommendations, $49 \%$ vs $49 \%$ felt that patients did not understand how to care for their diabetes.

Physician barriers : $31 \%$ vs $34 \%$ ( $p=N S$ ) reported difficulty adhering to clinical practice guidelines, $18 \%$ vs $25 \%$ found the choice of medications confusing, and $49 \%$ vs $9 \%(p<0.001)$ felt comfortable starting and titrating medications, while most residents considered hypoglycemia a barrier to diabetes control $(76 \%$ vs $65 \%, p=N S)$.

In conclusion, our study indicates that providing good diabetes care is challenging to physicians in training. Most residents reported significant healthcare system-, patient-, and physician-related barriers, which have improved little during the past decade. Diminishing resources for patient education need to be addressed to help overcome limitations related to patient understanding, literacy, and healthcare costs to ultimately affect better outcomes. 
715-P Speaking to Patients About Diabetes Risk: Is Terminology Important?

VALENTINA D. TARASOVA, JAIME A. CABALLERO, PAUL D. TURNER, SILVIO E. INZUCCHI, Omaha, NE, New Haven, CT

The natural history of Type 2 diabetes (T2D) typically includes years of mild hyperglycemia antecedent to diagnosis. There is lack of agreement between professional groups, however, as to the proper terminology to be used when referring to this stage. Moreover, there are no data on how such descriptions are perceived by patients who are being counseled regarding diabetes prevention. A more thorough understanding of this issue might assist clinicians in communicating more effectively with patients.

We invited 188 individuals with no history of diabetes, age 30-70 years old, to complete a written survey concerning 2 terms used to describe the atrisk for T2D state: 'prediabetes' and 'at high risk for diabetes'. A Likert scale was used to score responses regarding understanding and personal impact of the terms, as well as subsequent intention to adopt lifestyle changes were the description applied to them by their physician.

Subjects had a similar perception of the terms 'prediabetes' and 'at high risk for diabetes'. Responses regarding understanding and personal impact were essentially indistinguishable. The majority understood that these terms indicated the potential for the future development of diabetes $177.5 \%$ and $79.7 \%, p=.50$, respectively), and described this information as constituting a potentially important problem for them personally $(94.7 \%$ and $95.4 \%, p=.73$ ). Subjects also responded with similarly high motivation to adopt lifestyle modifications, such as diet (97.1\% vs. $96.7 \%, p=1.00)$, exercise $(96.4 \%$ vs. $95.9 \%, p=1.00)$ and weight loss $(89.8 \%$ vs. $92.2 \%, p=.58)$.

In this preliminary study, two common descriptions of the at-risk state for T2D appear to be similarly understood, impactful, and motivating for lifestyle change. Based on these data, clinicians can likely use either term interchangeably. More studies are needed, however, to determine whether use of one term versus another might actually be associated with greater adherence to lifestyle recommendations and, ultimately, diabetes prevention.

716-P

Intensification Behavior Is Key Feedback Component

DAVID C. ZIEMER, JANE M. CAUDLE, GUILLERMO E. UMPIERREZ, MARY RHEE, DAWN SMILEY, CHRISTOPHER NEWTON, VISHAL PAREKH, CINNAMON BRADLEY, JOLENE LOWERY, PAYAL S. SHAH, NKECHI MBAEZUE, CIRCE W. TSUI, CATHERINE S. BARNES, Atlanta, GA

Performance feedback has been shown to improve care delivered for diabetes in primary care. The crucial components of effective feedback are unclear. Observation of performance feedback in a municipal hospital primary care center suggest focusing on intensification behavior.

Diabetes registry data produced programmed feedback reports and datasets for multivariable regression analyses. Feedback reports and talking points were given to faculty of 2 medical schools who delivered performance feedback to 193 internal medicine residents in their respective continuity clinics. In both, general internists staffed patient care. In clinic IMC they also delivered feedback; in the other (EC), endocrinologists with more feedback experience delivered feedback. Scheduling limitations varied the number of sessions delivered (mean 60\%). On a survey $68 \%$ of physicians reported levels of $A 1 c$, random (RBG) or fasting (FBG) glucose at which they generally increased therapy (respective means $7.0 \%, 188$ and $136 \mathrm{mg} / \mathrm{dl}$ ).

After adjusting for year of training (PGY), a lower threshold of A1c for intensification was associated with receiving feedback $(0.3 \%)$ and EC $(0.25 \%)$ $(P<0.05)$, with a trend $(P=0.07)$. Intensification at clinic visits was associated with lower FBG thresholds, PGY, and EC after controlling for patient factors including glycemia; Feedback tended to improve intensification.

Lower subsequent A1c was associated with intensification, not being on insulin, and IMC, after adjusting for patient factors.

Better A1c levels were seen with more intensification and in IMC after adjusting for intensification. However, in EC, residents reported a lower threshold for intensification judging by A1c and in practice intensified more often.

Feedback increases frequency of intensification; experience or specialty may be factors in feedback effectiveness. Focusing physician behavior is more important than changing reported attitudes toward specific glycemic control goals. The key element to control A1c is taking action to intensify therapy
Is Diabetes Knowledge Affected by Timing of Continuous Subcutaneous Insulin Infusion (CSII) Therapy in Pediatric Patients?

EMILY FRYDMAN, SUSAN GARROW-SLOAN, ALEX KNEE, HOLLEY ALLEN, Springfield, $M A$

Initial intensive education during the fragile stage after T1DM diagnosis can be overwhelming. DK is essential to optimize diabetes management and quality of life. Initiating CSII early after diagnosis may overload a stressed family adjusting to a new diagnosis and hinder acquisition of sound DK.

Our objective was to evaluate the relationship between DK and early initiation of CSII therapy in patients with T1DM.

We retrospectively enrolled children and adolescents with T1DM followed in a moderate sized pediatric endocrinology program. Pt demographics, date of $\mathrm{dx}$, date of pump start and A1C were collected from medical records. Pts that were $>14$ yo and guardians of pts $<14$ yo completed a DK questionnaire evaluating basic understanding of diabetes pathophysiology (50), hypoglycemia (20), self diabetes care (40), nutrition (30) and CSII management (50). The primary outcome was DK score presented as \% correct (95\% CI). Primary analysis (controlled for confounders) assessed the relationship between DK and timing of transition to CSII.

Of 135 T1DM pts using CSII, $49 \%$ were female $(82 \% \mathrm{C}, 8 \% \mathrm{H}, 5 \% \mathrm{AA}, 2 \% \mathrm{~A}$ $3 \% 0$ ), mean age was $14.3 \pm 3.9$ (SD) yrs, mean time from $d x$ to CSII was $2.8 \pm$ 2.9(SD) yrs. Mean DK was $85.5 \%$ (83.7 to 87.2). Subjects were divided into 2 groups, 26 early CSII $(<6$ months after $\mathrm{dx}$ ) and 109 later CSII (>1yr). There was no difference between DK in early vs. late CSII, $89.5 \%$ (86.3 to 92.6) vs. $87.6 \%$ ( 85.7 to $89.5, p=0.35$ ). The older (>14yr) early CSII had slightly higher DK, 93\% (90.5 to 96.5) than the younger early CSII < 14 yr) DK, 88\% (83.7 to 92.4). Significantly higher DK was seen in older early vs. older late CSII, $93 \%$ (90.50 to 96.54 ) vs. $88 \%$ ( 86.47 to $90.80, p=0.028$ ) and Caucasian race vs. other race $(\mathrm{p}=<0.001)$.

In summary, older patients who transitioned early to CSII had slightly higher scores. Early initiation of CSII was not associated with lower DK which may reassure providers that early introduction of CSII does not impair acquisition of basic diabetes knowledge.

\section{EXERCISE-ANIMAL}

Guided Audio Tour: Animal Models to Interrogate Exercise Mechanisms (Posters: 718-P to 723-P), see page 17.

$\Delta$

NFkB Mediates Exercise-Induced Muscle Glucose Transport JIANBO WANG, NING ZHANG, JI LI, RALPH A. DEFRONZO, MENGYAO LI, STEVEN

E. SHOELSON, NICOLAS MUSI, San Antonio, TX, Boston, MA

Exercise increases glucose disposal into contracting muscles. Yet, the molecular mechanism by which exercise increases muscle glucose transport is unclear. Nuclear factor $-\kappa B$ (NFкB) is a family of transcription factors that control the gene expression of proteins involved in inflammation. Several groups, including ours, have shown that aerobic exercise increases NFKB activity in muscle from rodents and human subjects. However, the physiologic relevance of the changes in $\mathrm{NFKB}$ activity caused by exercise is not known In this study we tested the hypothesis that NFKB mediates exercise-induced muscle glucose transport. For this purpose, we studied transgenic mice whose $\mathrm{NF} \kappa \mathrm{B}$ activity is inhibited by overexpressing an I $\mathrm{\kappa B}$ superrepressor mutant in muscle (MISR). Ex vivo (isolated) muscle (soleus) 2-deoxyglucose transport was measured after $60 \mathrm{~min}$ of treadmill running $\left(22 \mathrm{~m} / \mathrm{min}, 15^{\circ}\right.$ incline) or 30 min of insulin stimulation $(50 \mathrm{mU} / \mathrm{ml})$. In wild type (WT) mice, exercise (ex) increased muscle glucose transport by 1.8-fold (WT sedentary: 3.2; WT ex: $5.8 \mu \mathrm{mol} / \mathrm{ml} . \mathrm{h}, \mathrm{P}<0.0001$, n=9/group), whereas exercise failed to stimulate glucose transport in MISR mice (MISR sed: 4.1; MISR ex: $4.6 \mu \mathrm{mol} / \mathrm{ml} . \mathrm{h}, \mathrm{P}=0.6$ $\mathrm{n}=9 / \mathrm{group})$. Insulin-stimulated glucose transport was similar in WT $(8.7 \mu \mathrm{mol} /$ $\mathrm{ml} . \mathrm{h})$ and MISR $(8.5 \mu \mathrm{mol} / \mathrm{ml} . \mathrm{h})$ mice (n=6/group). A graded exercise test was conducted to examine whether inhibition of NFKB affects exercise tolerance. However, there was no difference in maximal exercise tolerance between WT (107.5 $\pm 9.8 \mathrm{~min})$ and MISR (120.3 $\pm 21.4 \mathrm{~min})$ mice. In summary, (i) NFKB mediates exercise-induced muscle glucose transport; (ii) NFKB is not involved in glucose transport elicited by insulin stimulation; and (iii) alterations in exercise-induced glucose transport in MISR mice are not explained by changes in exercise tolerance. We conclude that the inflammatory response (i.e. NFKB activation) caused by acute muscle contraction plays a key role in regulating exercise-induced metabolic responses.

Supported by: NIA; NIDDK 
719-P

Physical Exercise Training and Metformin Treatment Attenuated Albuminuria and Shedding of ACE2 in $d b / d b$ Mice

HARI KRISHNA SOMINENI, ESAM SALEM, GREGORY BOIVIN, KHALID ELASED, Dayton, $\mathrm{OH}$

Angiotensin II (Ang II), a potent vasoconstrictor cleaved from Ang I, is responsible for the renal damage in diabetes. Studies have shown that the strict glycemic control and blockade of renin angiotensin system attenuates diabetic kidney damage. Angiotensin converting enzyme (ACE) 2 is highly expressed in the kidney and has been shown to be renoprotective by degrading Ang II to Ang-(1-7). We have shown previously increased urinary ACE2 excretion in $d b / d b$ type 2 diabetic mice which contributes to the pathogenesis of diabetic nephropathy. We tested the hypothesis that physical exercise training and metformin treatment improve glucose homeostasis and attenuate albuminuria in $d b / d b$ mice. We also investigated whether there is a correlation between urinary albumin excretion and shedding of ACE2 in diabetic mice. Six wks old normal and $d b / d b$ mice were subjected either to physical exercise training and/or metformin treatment (150 mg/kg/day) for 10 wks. The exercise groups were run on a mouse forced exercise walking wheel system for 1 hour a day for 7 days a wk at a speed of $8 \mathrm{~m} / \mathrm{min}$. Exercise training significantly lowered blood glucose, urinary albumin and ACE2 excretion $(p<0.05)$ in $d b / d b$ mice. Furthermore, exercise training lowered food and water intake of $d b / d b$ mice, but had no effect on their body weights. Metformin treatment decreased hyperglycemia, urinary albumin and ACE2 excretion ( $p<0.05)$ only during the first 2 wks of treatment. Further, urinary ACE2 correlated positively with, albuminuria $(r=0.84 ; p<0.0001)$, glycemia $(r=0.75 ; p<0.0001)$, plasma glucagon $(r=0.66$; $p<0.001)$ and triglycerides $(r=0.75 ; p<0.0001)$. In addition, exercise training reduced plasma triglycerides and enhanced insulin levels in $d b / d b$ mice. In conclusion, physical exercise training attenuated microalbuminuria and shedding of renal ACE2 throughout the study. However, metformin was only effective during the initial stages of diabetes.

ก 720-P Nitric Oxide Is Required for Increases in Insulin Sensitivity following Ex Vivo Contraction of Mouse Skeletal Muscle

MARY X. ZHANG, STEPHEN RATTIGAN, GLENN MCCONELL, ANTHONY ZULLI, ALAN HAYES, Melbourne, Australia, Tasmania, Australia

It is well-known that exercise training is very effective in people with Type 2 Diabetes (T2D). Furthermore, a single bout of exercise increases skeletal muscle insulin sensitivity. However, the factors regulating skeletal muscle glucose uptake are incompletely understood. Nitric oxide (NO) plays an important role in the increase in skeletal muscle glucose uptake during exercise in rodents and humans. However, whether NO production during acute contraction/exercise is critical for the consequential increase in insulin-stimulated glucose uptake after contraction/ exercise is unknown.

To address this question, isolated extensor digitorum longus (EDL) muscle from C57BL/6J mice were stimulated ex vivo to contract for $10 \mathrm{~min}$ (in the presence or absence of the NO synthase (NOS) inhibitor $\mathrm{N}^{\mathrm{G}}$-monomethyl-Larginine (L-NMMA)). Paired EDL were incubated with $\left[{ }^{3} \mathrm{H}\right]-2$-deoxyglucose and $\left[{ }^{14} \mathrm{C}\right]-$ mannitol with or without insulin $(120 \mu \mathrm{U} / \mathrm{ml})$ to measure glucose uptake post contraction. In the no insulin condition, $3.5 \mathrm{hr}$ after contraction skeletal muscle glucose uptake was not different to basal and NOS inhibition had no effect on basal skeletal muscle glucose uptake. Contraction had a significant insulin-sensitizing effect on skeletal muscle glucose uptake 3.5 h post contraction ( 2.9-fold increase, $\mathrm{p}<0.01)$ compared with insulin alone $(\sim 57 \%$ increase, $p<0.01)$. NOS inhibition significantly blunted this increase in insulin-stimulated glucose uptake $(\sim 2.3$-fold decrease, $p<0.05)$.These results demonstrate that $\mathrm{NO}$ is essential for the post-contraction increase in insulin sensitivity (glucose uptake) in mouse skeletal muscle.

Supported by: NHMRC (Australia) Decreased Expression of Micro-RNA-494 during Muscle Differentiation and Exercise Stimulate Mitochondrial Biogenesis

LUCIA MOROTO, KATSUTARO MORINO, HIROTAKA YAMAMOTO, OSAMU SEKINE, TAKESHI YOSHIZAKI, HIROTAKA IWASAKI, TAKESHI IMAMURA, SATOSHI UGI, ATSUNORI KASHIWAGI, HIROSHI MAEGAWA, Otsu, Japan

MicoRNAs (miRNAs) are important post-transcriptional regulators of various biological pathways. The role of miRNAs during mitochondrial biogenesisin skeletal muscle remains unclear especially in vivo. Microarray based screening was performed to find novel miRNAs related with skeletal muscle differentiation. We have found that the expression of miR-
494 markedly decreased in murine myoblast C2C12 cells during myogenic differentiation, accompanied by an increase in mitochondrial DNA. Also, the expression of predicted target genes for miR-494, mitochondria transcriptional factor A (mtTFA) was post-transcriptionally increased during myogenic differentiation. Inhibition of miR-494 by a specific antisense resulted in increase in mitochondrial content and upregulated level of mtTFA at the protein level. In addition, overexpression of miR-494 decreased protein expression of mtTFA in C2C12 cells. Furthermore, the miR-494 content significantly decreased after endurance exercise in $\mathrm{C} 57 \mathrm{BL} / 6 \mathrm{~J}$ mice, accompanied by an increase in expression of the mtTFA. Finally, in vivo overexpression of miR-494 mimic by direct injection into pretibial muscle significantly decreased the protein expression of mtTFA. In conclusion, these results suggest that miR-494 regulates mitochondrial biogenesis by downregulating mtTFA during myocyte differentiation and skeletal muscle adaptation to physical stimulation.

ก 722-P

Exercise-Mediated Reductions in Blood Glucose Remain Unchanged Following Aerobic Training in Moderately Hyperglycemic Type 1 Diabetic Mellitus Rats

MATTHEW W. MCDONALD, KENNETH N. GRISE, T. DYLAN OLVER, ADWITIA DEY, J. KEVIN SHOEMAKER, EARL G. NOBLE, C.W. JAMES MELLING, London, ON, Canada

It is well recognized that an acute bout of exercise can elicit a hypoglycemic episode in Type 1 diabetes mellitus (T1DM) patients. This study examined exercise-mediated reductions in blood glucose and the glucoregulatory response of glucagon, in moderately hyperglycemic T1DM rats. Further, it was determined if 10 weeks of aerobic training could alleviate these exercise-mediated reductions in blood glucose through an increase in serum glucagon and/or hepatic glycogen levels, the primary source of blood glucose during exercise. Thirty-two rats were equally divided into four groups: non-T1DM sedentary, T1DM sedentary, non-T1DM exercised or T1DM exercised. Exercise consisted of treadmill running, 5 days/week $(27 \mathrm{~m} /$ min; $6 \%$ grade; 1 hour) for 10 weeks. T1DM was induced via 5 consecutive days of streptozotocin (STZ) injections $(20 \mathrm{mg} / \mathrm{kg} ; 0.1 \mathrm{M}$ Citrate buffer, $\mathrm{pH}$ 4.5), followed by implantation of insulin pellets to maintain blood glucose in a moderately hyperglycemic range $(9-15 \mathrm{mmol} / \mathrm{L})$. At week 1 , an acute exercise bout led to a significant reduction in blood glucose in T1DM rats in comparison to non-T1DM rats $(p<0.05)$, while serum glucagon levels were not different between T1DM and non-T1DM rats ( $p>0.05)$. Following 10 weeks of training, neither the exercise-mediated reduction in blood glucose or serum glucagon levels in response to exercise were altered. Interestingly, hepatic glycogen levels in T1DM rats at 10 weeks of training were significantly lower than non-T1DM rats $(p<0.001)$ and were not different in comparison to T1DM sedentary rats $(p>0.05)$. It summary, moderately hyperglycemic T1DM rats demonstrated lower hepatic glycogen levels in comparison to non-T1DM, which is unaltered following training. Lowered hepatic glycogen levels may explain, in part, the immediate reduction in blood glucose in response to acute exercise and the inability of aerobic training to improve these exercisemediated reductions in glycemia.

Supported by: CCIHR (217532)

Low Intensity Vibrations Simultaneously Improve Type 2 Diabetes and Bone Quality in Obesity Potentially via Influencing Bone Marrow Mesenchymal Stem Cell

M. ETE CHAN, DANIELLE GREEN, BENJAMIN ADLER, GABRIEL PAGNOTTI, DENIS NGUYEN, JAMES LENNON, VIHITABEN PATEL, CLINTON T. RUBIN, Stony Brook, NY

Obesity has been recognized as a chronic inflammation status that increases the risk of both type 2 diabetes (T2D) and bone fracture. Although exercise is effective in improving these obesity-induced complications, it is still unclear how mechanical signals, a critical element of exercise, exert their salutary effects. A potential candidate that mechanical signals can act to treat the skeletal and immune system dysfunction simultaneously is the mesenchymal stem cell (MSC), which is the progenitor of bone-forming cells and possesses an immunomodulatory property. To examine the effect of mechanical signals on the role of MSC in improving glucose tolerance and bone quality in obesity, $305 \mathrm{w}$ old male C57BL/6 $\mathrm{J}$ mice were divided into 3 groups ( $n=10)$ : regular $\operatorname{diet}(\mathrm{RD})$, high fat diet (HF) and high fat diet with low intensity vibrations (HFv). Low intensity vibrations (LIVS) were mechanical signals delivered via a vertically oscillating plate $(30 \mathrm{~min} /$ day, 5 day/wk at $90 \mathrm{~Hz}$ and $0.2 \mathrm{~g}$ ) to $\mathrm{HFv}$, while $\mathrm{HF}$ and $\mathrm{RD}$ were sham-handled. While HF exhibited clear signs of glucose intolerance after $8 \mathrm{w}$ of high fat diet than RD 


\section{EXERCISE-HUMAN}

$(+15 \%, p=0.04)$, LIVs normalized glucose intolerance in HFv towards RD (only $+4 \%$, nsd), indicating that LIVs ameliorated T2D in HFv. Interestingly, the status of T2D is changed along with the mechanical property of bone. The tibiae of HF had lower stiffness (-22\%) and elastic modulus (-45\%) than RD $(p=0.03)$, indicating an increased fracture risk in obesity. On the other hand, LIVs increased these properties of HFv by $+27 \%$ and $+108 \%$ compared to HF $(p=0.02)$, respectively. In the cellular and molecular levels, LIVs increased the bone marrow MSC populations by $+53 \%$ and reduced the expression of TLR4, a pro-inflammatory marker of MSC, by $-25 \%$ in HFv relative to HF $(p<0.05)$. LIVs may serve as the simultaneous treatment of both compromised skeletal system and T2D by modulating the number and the immunomodulatory property of bone marrow MSC.

Supported by: AR49438; EB14351

724-P

The Effect of Exercise Modality on the Ventricular Myocardial HSP70 Expression in a Type 1 Diabetic Rat Model

ADWITIA DEY, MATTHEW W. MCDONALD, KATHARINE E. HALL, MAO JIANG,

EARL G. NOBLE, C.W. JAMES MELLING, London, ON, Canada

Type One Diabetes Mellitus (T1DM) is an endocrine disorder that may lead to myocardial dysfunction. The purpose of this study was to investigate the impact of 6-weeks of aerobic or resistance exercise training on cardiac expression and localization of the cardioprotective Hsp70 protein in insulintreated streptozotocin (STZ) induced T1DM rats. Five groups ( $\mathrm{N}=10$ each) of animals were used, including 1 control (C) and 4 T1DM groups (administered $5-7$ daily doses of $20 \mathrm{mg} / \mathrm{kg}$ until 2 consecutive blood glucose measures of greater than $18 \mathrm{mmol} / \mathrm{L}$ were attained). In order to mimic a "poorly controlled" diabetic state (9-15 mmoles/l blood glucose), insulin pellets were implanted subcutaneously for all diabetic groups. Three T1DM groups were subsequently exercised via either treadmill running at high-intensity (DH)-(70-80\% V02Max) or low-intensity (DL)-(50-60\% V02Max), or resistance trained (DR) for 6 weeks, 5 days a week. Ventricular Hsp70 protein levels and localization were quantified by western blotting and fluorescent immunohistochemistry (IHC) respectively. Compared to $\mathrm{C}$ and control diabetic (CD) groups, exercise trained animals exhibited higher myocardial Hsp70 expression ( $p<0.05)$. Hsp70 levels were higher $(p<0.05)$ in $\mathrm{DR}$ and $\mathrm{DH}$ when compared to $\mathrm{DL}$ animals. IHC data demonstrates a distinct vascular localization of Hsp70 in both DR and DH which was most prominent in DR. This is the first study to examine the effect of resistance training on Hsp70 content in the myocardium of T1DMs. It demonstrated that the vasculature, particularly with resistance training, was a prime location for increases in cardioprotective Hsp70. It further confirmed that only high intensity exercise (resistance or aerobic) were sufficiently stressful to induce significant increases in Hsp70. These data suggest that resistance exercise as well as aerobic high intensity aerobic training may induce the cardioprotective Hsp70 in T1DM myocardium.

Supported by: CIHR (CCT83029)

725-P

Preventing Exercise-Induced Hypoglycemia via Enhanced Glucagon Release in a Rodent Model of Type 1 Diabetes

ERWAN LECLAIR, ASHLEY PECKETT, EMILY DUNFORD, TREVOR TEICH, RICHARD LIGGINS, DAVID COY, MLADEN VRANIC, MICHAEL C. RIDDELL, Toronto, ON, Canada, Vancouver, BC, Canada, New Orleans, LA

Pancreatic and/or circulating somatostatin levels are elevated in humans with diabetes and in rodent models of the disease. We have recently established that this inhibition can be alleviated by the use of a somatostatin type 2-selective receptor antagonist (PRL-2903) in STZ-diabetic rodents. The purpose of this study is to test the efficacy of PRL-2903 administration on glucagon counterregulation during exercise-induced hypoglycemia in diabetic rats. Ten hyperglycemic STZ-diabetic (D) rats were injected i.p. either with saline or with a $10 \mathrm{mg} / \mathrm{kg}$ dose of PRL-2903 (60 min before exercise) in a randomized counterbalanced cross-over design, followed 30 min later with an insulin injection that would normally target euglycemia at rest (Regular insulin given s.q. on a sliding scale based on historical data from these same rats). Running exercise at $17 \mathrm{~m} / \mathrm{min}$ and $0 \%$ slope on a rodent treadmill began 30 minutes later ( $T=0 \mathrm{~min}$ ) and lasted for 30 minutes to induce mild hypoglycemia (2.5-3.5 mM). Blood glucose (BG) levels were measured periodically and blood was collected via saphenous vein bleed for glucagon levels at baseline, after exercise ( $\mathrm{T}=30 \mathrm{~min}$ ) and in recovery $(\mathrm{T}=75 \mathrm{~min})$. The nadir in glucose occurred at $\mathrm{T}=60 \mathrm{~min}(3.0 \pm 1.5$ vs. $2.7 \pm 1.2$ $\mathrm{mM}$ ) in the PRL-2903 and saline treated rats, respectively. Baseline glucagon concentrations were not different between trials ( $91 \pm 21 \mathrm{vs.} 89 \pm 28 \mathrm{pg} /$ $\mathrm{mL})$. However, by the end of exercise, glucagon levels increased more than 2 -fold with PRL-2903 $(P<0.05)$ but remained unchanged with saline $(226 \pm 122$ $\mathrm{pg} / \mathrm{mL}$ vs. $116 \pm 35 \mathrm{pg} / \mathrm{mL}$ at $\mathrm{T}=30 \mathrm{~min})$. In recovery, glucagon values tended to be higher in the PRL-2903 vs. saline treated group ( $134 \pm 83 \mathrm{vs.} 80 \pm 33 \mathrm{pg} /$ $\mathrm{mL}$ in PRL-2903 vs. saline at T=75 min) as glucose was normalized (5.5 \pm 1.0 vs. $4.1 \pm 0.8$ ). As previously demonstrated in non-exercising rats with insulininduced hypoglycemia, we show here that PRL-2903 improves the glucagon response to exercise-induced hypoglycemia in diabetic rats.

Endurance Exercise Training Improves Insulin-Stimulated Glucose Transport and Tyrosine Phosphorylation of IRS-1 but Not PI3-Kinase and Akt in Skeletal Muscle of Ovariectomized Rats

VITOON SAENGSIRISUWAN, MUJALIN PRASANNARONG, KANOKWAN VICHAI-

WONG, Bangkok, Thailand, Chiangmai, Thailand, Nakhonnayok, Thailand

Insulin resistance of skeletal muscle glucose transport due to prolonged loss of ovarian function in ovariectomized (OVX) rats is accompanied by other features of the metabolic syndrome. We have previously demonstrated that endurance exercise training prevents insulin resistance in skeletal muscle of OVX rats. However, the potential cellular mechanisms that underlie the beneficial adaptations remain to be elucidated. To address this issue, the present study assessed the protein expression and functionality of specific elements known to regulate the glucose transport process including $I R$ IRS-1, the p85 subunit of PI3-kinase, Akt, Akt substrate of $160 \mathrm{kDa}$ (AS160), AMP-activated protein kinase-alpha (AMPK- $\alpha$ ), and GLUT-4 in OVX rats that remained sedentary, performed exercise training $(\mathrm{ET})$, received $2.5 \mu \mathrm{g} / \mathrm{kg}$ of $17-\beta$ estradiol (E2), or underwent both treatments. We found that ET or E2 individually improved insulin action on skeletal muscle glucose transport (36-65\%) which was accompanied by significant increase in GLUT-4 protein abundance $(61-80 \%)$. E2 effectively restored the defects in the insulin signaling cascade while ET improved tyrosine phosphorylation of IRS-1 (74\%) but not other downstream signaling elements. The combined treatment led to an additive effect on insulin-stimulated glucose transport. However, no interactive effects of ET and E2 on the signaling proteins were observed. Conclusively, the present investigation provided evidence that the insulin sensitizing effects of $\mathrm{ET}$ on the insulin signaling pathway in skeletal muscle of OVX rat is limited to the enhanced tyrosine phosphorylation of IRS-1 and does not bring about adaptations of the downstream insulin signaling elements. Moreover, the interactive effects of ET and E2 on skeletal muscle insulin action in $\mathrm{VVX}$ rats are not responsible for by adaptations of signaling proteins determined in the present study.

Supported by: Thailand Research Fund (RMU-5380010)

\section{EXERCISE-HUMAN}

Guided Audio Tour: Exercise in Humans-Efficacy and Possible Mechanisms (Posters: 727-P to 732-P), see page 17.

Cardiorespiratory Fitness and its Impact on Mortality in Prediabetics DAVID NI, MANCHIN CHANG, ERIC S. NYLEN, CHARLES FASELIS, JONATHAN MYERS, PETER F. KOKKINOS, Washington, DC, Palo Alto, CA

The CDC estimates that 79 million Americans (or approximately 1 in 3) have prediabetes, with an associated risk of developing type 2 diabetes, heart disease, stroke, and death. Despite enhanced lifestyle adherence, there is a paucity of data regarding mortality outcome.

The objective of this study was to evaluate the role of cardiorespiratory fitness (CRF) on mortality in prediabetic veterans.

In a cohort of 1118 prediabetic veterans (mean age 59), CRF was calculated based on peak metabolic equivalents (METs) obtained from routine exercise tolerance testing. Based on METS, four fitness categories were established; Low-Fit (<5.8 METs; lower 25th percentile), Mild-Fit (5.8-7 METs), ModerateFit (7.1 - 8.5 METs), and High-Fit (>8.5 METs; > 75th percentile). Date of death was verified from the Veterans Affairs Beneficiary Identification and Record Locator System File.

In a mean follow-up period of 7.7 years ( 8,610 person-years) there were a total of 251 deaths, averaging 29.1 events per 1000 person-years. An inverse and graded association between exercise capacity and mortality risk was observed ( $p=0.002$ for trend). For every 1-MET increase in exercise capacity, the adjusted mortality was lowered by $13 \%$ (hazard ratio $=0.87 ; \mathrm{Cl}, 0.81$ $0.94, p<0.001)$. The Mild, Moderate, and High-Fit subjects had significantly reduced mortality by $32 \%$ (hazard ratio $=0.68 ; \mathrm{Cl}, 0.50-0.94, \mathrm{p}=0.02$ ), $40 \%$ (hazard ratio $=0.60 ; \mathrm{Cl}, 0.41-0.87, \mathrm{p}=0.007$ ) and $54 \%$ (hazard ratio $=0.46 ; \mathrm{Cl}$, $0.30-0.70, p<0.001$ ) respectively. 
In conclusion, a strong inverse and graded association between CRF and mortality risk was observed in these prediabetic veterans. Mortality risk was $13 \%$ lower for every 1-MET increase in CRF and approximately $50 \%$ lower in High-Fit individuals (exercise capacity > 8.5 METs) compared to Least Fit individuals. With increasing incidence of diabetes as well as diminished response to preventative lifestyle modifications, enhanced CRF should be advocated in prediabetic individuals.

\section{ก 728-P}

Exercise or Habitual Physical Activity to Improve Blood Glucose Homeostasis in Patients With Type 2 Diabetes?

JAN-WILLEM VAN DIJK, MAARTEN VENEMA, WILLEM VAN MECHELEN, COEN

D. STEHOUWER, FRED HARTGENS, LUC J. VAN LOON, Maastricht, Netherlands, Amsterdam, Netherlands

In the present study we investigated the impact of repeated bouts of nonexercise habitual physical activity versus endurance-type exercise on daily blood glucose homeostasis in type 2 diabetic patients

Twenty type 2 diabetic patients participated in a randomized crossover study, consisting of 3 experimental periods separated by 1 week. Twentyfour-hour glycemic control was assessed under sedentary control conditions, and under conditions where prolonged sedentary time was reduced either by three 15-min bouts of habitual physical activity per day (postmeal strolling, $~ 3$ metabolic equivalents [MET]) or by a single 45 -min bout of moderate-intensity endurance-type exercise ( 6 MET). Blood glucose profiles were assessed by continuous glucose monitoring, and plasma insulin concentrations were determined in frequently sampled venous blood samples.

Hyperglycemia (blood glucose $>10 \mathrm{mmol} / \mathrm{L}$ ) was experienced for 6:51 $\pm 1: 04$ $\mathrm{h}$ :min per day during the sedentary control condition, and was significantly reduced by exercise $(4: 47 \pm 1: 02 \mathrm{~h}: \mathrm{min}, \mathrm{p}<0.001)$, but not by habitual physical activity (6:02 $\pm 1: 16 \mathrm{~h}: \mathrm{min}, p=0.67)$. The cumulative glucose incremental AUC of breakfast, lunch and dinner, was respectively $35 \pm 5 \%(p<0.001)$ and $17 \pm 6 \%(p<0.05)$ lower during the exercise and habitual physical activity condition compared with the sedentary condition. The insulin incremental AUCs were respectively $33 \pm 4 \%(p<0.001)$ and $17 \pm 5 \%(p<0.05)$ lower during the exercise and habitual physical activity conditions compared with the sedentary condition.

When matched for total duration, moderate-intensity endurance-type exercise represents a more effective strategy to improve daily blood glucose homeostasis than repeated bouts of habitual physical activity. Nevertheless, increasing the daily amount of habitual physical activity represents a valuable strategy to improve postprandial glucose handling in patients with type 2 diabetes.

\section{ก 729-P}

Effects of Exercise Amount and Intensity versus Lifestyle Intervention on Glucose Tolerance in Adults With Prediabetes-STRRIDE PD

CRIS A. SLENTZ, LORI A. BATEMAN, LESLIE H. WILLIS, ESTHER GRANVILLE, LUCY W. PINER, LORRIE ELLIOT-PENRY, CONNIE W. BALES, WILLIAM E. KRAUS, Durham, NC

While exercise robustly improves glucose tolerance, the optimal intensity or amount of exercise for improving glucose tolerance is not known. We compared 3 6-month exercise-only groups differing in amount and/ or intensity to a diet + exercise group similar $t o$ the first 6 months of the DPP. Adults (45-75y) with prediabetes were randomized to: 1) Low-Amount/ Moderate-Intensity exercise $\left(10 \mathrm{Kcal} / \mathrm{Kg} / \mathrm{wk}\right.$ at $50 \%$ peak $\left.\left.\mathrm{VO}_{2}\right) ; 2\right)$ HighAmount/Moderate-Intensity exercise $\left(16 \mathrm{Kcal} / \mathrm{Kg} / \mathrm{wk}\right.$ at $50 \%$ peak $\left.\mathrm{VO}_{2}\right)$; 3) High-Amount/Vigorous-Intensity $\left(16 \mathrm{Kcal} / \mathrm{Kg} / \mathrm{wk}\right.$ at $75 \%$ peak $\left.\mathrm{VO}_{2}\right)$; and 4) Diet + Exercise - goal of 7\% weight loss and Low-Amount/ModerateIntensity exercise (same as group 1). An OGTT was administered pre and post intervention, with 117 subjects with full OGTT data ( 30 per gro up). RESULTS: The Low-Amt /Mod-Intensity group achieved $55 \%$ of the Diet + Exercise glucose lowering effect. Two major findings were surprising and remarkable: 1) Six months of moderate intensity exercise was significantly better than the same amount of vigorous intensity exercise at improving glucose tolerance - with 6 times the effect; 2) The High-Amt/Mod-Intensity group was as effective (98.9\% of total effect) as the Diet + Exercise group (the DPP-like intervention), implying that the entire 6-month DPP effect of exercise, diet and $7 \%$ weight loss on glucose tolerance in adults with prediabetes can be achieved with moderate intensity exercise alone.

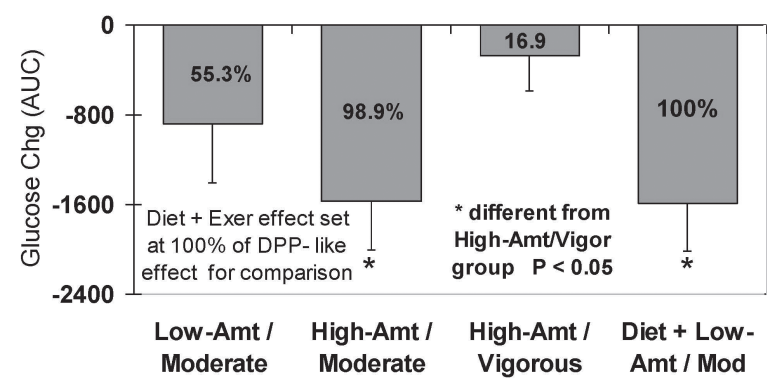

Supported by: 2R01-HL05354

ก 730-P

Peripheral but Not Hepatic Insulin Sensitivity Increases during Moderate Exercise in the Postprandial State: A Triple Tracer Study LING HINSHAW, MICHELE SCHIAVON, CHIARA DALLA MAN, ASHWINI MALLAD, RITA BASU, YOGISH C. KUDVA, CLAUDIO COBELLI, ANANDA BASU, Rochester, MN, Padova, Italy, Padua, Italy

Exercise improves insulin sensitivity. However, measurement of peripheral insulin sensitivity on glucose disappearance (Rd) and hepatic insulin sensitivity on endogenous glucose production (EGP) during exercise in the postprandial state has not yet been developed in humans. To do so, we studied 12 healthy subjects ( 5 men, age $37.1 \pm 3.1 \mathrm{yrs}, \mathrm{BMl} 24.1 \pm 1.1 \mathrm{~kg} / \mathrm{m}^{2}$, FFM $50.9 \pm 3.9 \mathrm{~kg}$ ) during moderate exercise at $50 \%$ V02 max for 75 minutes, 120-195 min after a triple tracer mixed meal consumed at time $0(76.6 \pm 0.3$ grams carbs; $48 \%$ carbs, 33\% fat, $19 \%$ protein; $671.1 \pm 58.8 \mathrm{kcall}$. Tracer infusion rates were adjusted to achieve constant tracer-tracee ratio and minimize nonsteady state errors. Peripheral and hepatic insulin sensitivities were estimated as the effect of insulin on Rd (iAUC Rd/iAUC insulin) and EGP (iAUC EGP/iAUC insulin) respectively in each subject both in presence ( $\mathrm{t}=0$ $360 \mathrm{~min}$ ) and absence of exercise ( $\mathrm{t}=0-120 \mathrm{~min})$. Plasma glucose rose from $4.9 \pm 0.2$ to a peak of $9.1 \pm 0.3 \mathrm{mM}$ at $\mathrm{t}=60$, fell to a nadir of $3.9 \pm 0.3$ at $\mathrm{t}=140$ before rising to $5.1 \pm 0.1 \mathrm{mM}$ at $\mathrm{t}=360 \mathrm{~min}$. Plasma insulin rose from $3.7 \pm 0.7$ to a peak of $54.1 \pm 6.8 \mu \mathrm{U} / \mathrm{ml}$ at $\mathrm{t}=60$, fell to $32.8 \pm 6.8$ at $\mathrm{t}=120$, then dropped rapidly to $4.9 \pm 1.1 \mu \mathrm{U} / \mathrm{ml}$ at $\mathrm{t}=195$, before reaching $4.5 \pm 1.4 \mu \mathrm{U} / \mathrm{ml}$ at $\mathrm{t}=360 \mathrm{~min}$. While peripheral insulin sensitivity was higher $(1.76 \pm 0.4 \mathrm{vs} .1 .38 \pm 0.2 \mathrm{\mu mol} /$ $\mathrm{kg}$ per $\mu \mathrm{U} / \mathrm{ml} ; \mathrm{p}=0.01)$, hepatic insulin sensitivity did not change $(-0.28 \pm 0.08$ vs. $-0.28 \pm 0.04 \mu \mathrm{mol} / \mathrm{kg}$ per $\mu \mathrm{U} / \mathrm{ml} ; p=0.97)$ in the presence vs. absence of exercise respectively. In conclusion, with state of the art tracer methods, we show that postprandial peripheral but not hepatic insulin sensitivity is increased during moderate exercise in healthy subjects. Further studies with higher intensity exercise including in people with diabetes could have multiple translational applications such as closed-loop control for type 1 diabetes.

Supported by: NIDDK (DK085516), (DKDP3-094331)

Training Alters Skeletal Muscle DAG Accumulation During Modest FFA Elevation

LISA CHOW, DOUGLAS G. MASHEK, LYNN E. EBERLY, ELIZABETH SEAQUIST, MICHAEL D. JENSEN, Minneapolis, MN, St. Paul, MN, Rochester, MN

Free fatty acid (FFA) elevation by lipid infusion is an established model to induce insulin resistance. We previously reported that modest FFA elevation by lipid infusion produced comparable reductions in insulin sensitivity in trained (T) \& sedentary (S) volunteers. We hypothesized this reduction was due to lipid infusion effects on muscle triacylglycerol (TAG), diacylglycerol (DAG) \& ceramide composition.

14 trained \& 14 sedentary volunteers matched for age, sex \& BMI received either a 6 hour lipid (L: $20 \%$ Intralipid: $90 \mathrm{ml} / \mathrm{h}$ no heparin) or glycerol (G:2.25 $\mathrm{g} / 100 \mathrm{ml}$ at $90 \mathrm{ml} / \mathrm{h}$ ) infusion during a hyperinsulinemic-euglycemic clamp. 3 muscle biopsies were taken (Bx1: 0 min, Bx2: 120 min, Bx3: 360 min). TAG, DAG \& ceramide composition were measured using liquid chromatography/ tandem mass spectrometry.

As expected, trained volunteers had higher V02max \& insulin sensitivity, as well as greater fat free mass. With lipid infusion, FFA concentrations $(616$ - $694 \mu \mathrm{mol} / \mathrm{L})$ \& decline in insulin sensitivity $[-48 \%(\mathrm{~T})$ vs $-47 \%$ (S)] were comparable in trained \& sedentary subjects.

Results are shown in Fig 1. Lipid infusion did not change total TAG (Fig1A) but increased 18:2 \& 18:3 in TAG (Fig1B-1C) for both groups. In the sedentary group, total DAG (Fig1D) \& saturated DAG (Fig1E) were greater with lipid infusion than glycerol infusion. In the trained group, saturated ceramides (Fig1F) declined with glycerol infusion. 
We conclude that training alters muscle DAG accumulation during modest FFA elevation.

Figure 1: Training effects on muscle TAG, DAG and ceramide composition during modest FFA Elevation

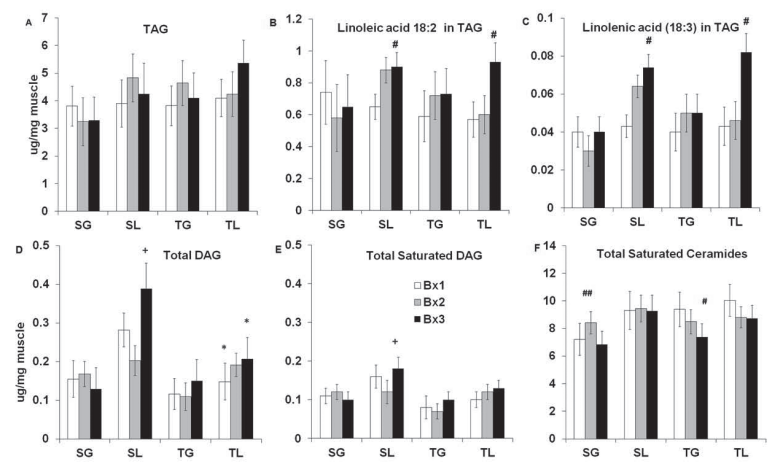

\# indicates significant difference $\mathrm{c} / \mathrm{w} \mathrm{Bx} 1$

+ indicates significant difference between lipid and glycerol infusion within same training status *indicates significant difference between trained and sedentary equivalents \#\# indicates significant difference between $\mathrm{Bx} 1$ and $\mathrm{B} \times 3$

Total saturated DAG defined as sum of 14:0, 16:0, 18:0 in DAG

Total saturated ceramides defined as sum of 14:0,16:0, 18:0 \&20:0 in ceramides

SG:Sedentary Glycerol, SL: Sedentary Lipid, TG:Trained Glycerol, TL:Trained Lipid

Supported by: 5K12RR023247-02

732-P

Effects of Physical Activity on Nocturnal and Next Day Hypoglycemia in Adolescents With Type I Diabetes

KRISTEN M. METCALF, AJAY SINGHVI, EVA TSALIKIAN, MICHAEL TANSEY, MIRIAM B. ZIMMERMAN, DALE ESLIGER, KATHLEEN JANZ, lowa City, IA, Loughborough, United Kingdom

Physical activity (PA) provides many benefits to adolescents with Type 1 Diabetes (T1D); however, adolescents with T1D tend to have lower fitness and PA levels. One reason adolescents with T1D engage in less PA is due to a fear of hypoglycemia. Most studies examining PA in relation to glycemic control measure PA through self-report, thus introducing bias. The purpose of this study was to objectively monitor PA and glucose in adolescents with T1D to examine the temporal associations between moderate and vigorous intensity physical activity (MVPA) and hypoglycemia.

Twenty participants ( 14 to $19 \mathrm{yr}, \mathrm{n}=10$ females and 10 males) with a T1D diagnosis for at least 1 year were recruited. Participant fitness was evaluated via indirect calorimetry during a maximal treadmill exercise test, and body composition was measured using air displacement plethysmography. An accelerometer (GENEActiv, Activinsights Ltd, Kimbolton, UK) was worn on the wrist continuously for 7 days and the waveform data used to estimate MVPA in min/d. Blood glucose values were simultaneously tracked using continuous glucose monitoring (DexCom SEVEN PLUS, San Diego, CA). After controlling for gender, $\%$ body fat (\%BF), and fitness, the likelihood of hypoglycemia ( $\leq 70 \mathrm{mg} / \mathrm{dl}$ ) at nighttime or the next day due to MVPA was examined using logistic regression.

Participants were of avg fitness (females: $43.9 \mathrm{ml} / \mathrm{kg} / \mathrm{min}$; males: 49.8 $\mathrm{ml} / \mathrm{kg} / \mathrm{min}$ ) and fatness (females: $26.2 \%$; males: $19.2 \%$ ), and $63.2 \%$ of participants met the US federal guidelines of accumulating $60 \mathrm{~min} / \mathrm{d}$ of MVPA. Hypoglycemia was $15 \%$ more likely in those who had $30 \mathrm{~min} / \mathrm{d}$ more MVPA than those with less (95\% Cl: 1.02, 1.30; $p=0.022$ ).

The results indicate that participating in MVPA increases the risk of hypoglycemia during the night time and the following day. The relationship is independent of gender, \%BF and fitness. While promoting PA as a healthy behavior, it is important to educate adolescents with T1D on prevention of hypoglycemia following PA.

Supported by: NIH (2UL1TR000442-06)
733-P

Estimation of Postprandial Insulin Sensitivity during Moderate Exercise in Healthy Subjects: Validation Against Meal Triple Tracer Technique

MICHELE SCHIAVON, CHIARA DALLA MAN, LING HINSHAW, ASHWINI MALLAD, RITA BASU, ANANDA BASU, YOGISH C. KUDVA, CLAUDIO COBELLI, Padova, Italy, Padua, Italy, Rochester, MN

Physical activity modulates insulin sensitivity. However, measurement of postprandial insulin action applying state of the art modeling methods in the context of physical activity during the same time period has not been developed in humans.

To do so and validate against the triple tracer technique, we studied 12 healthy subjects who underwent a triple tracer mixed meal, containing 75 $\mathrm{g}$ of $\mathrm{CHO}$ and performed moderate physical activity (50\% V02 max) for 60 minutes during a 75 minute period, starting 120 min after the meal. Plasma glucose and insulin concentrations were used to estimate insulin sensitivity with the integral formula. Insulin sensitivity was calculated, both in absence (i.e. for $\mathrm{t}=0-120 \mathrm{~min}, S_{I}^{\text {rest }}$ ) and presence of physical activity $(\mathrm{t}=0-360 \mathrm{~min}$, $S_{1}^{e x}$. In addition, endogenous glucose production (EGP) and rate of glucose disappearance $\left(R_{d}\right)$ were estimated with the triple tracer technique. This permitted calculation of glucose disposal, liver and total insulin sensitivity from area under EGP and $\mathrm{R}_{\mathrm{d}}$, both in absence (TTS $S_{l}^{\text {resto }}, \pi S_{l}^{\text {restl }}, \pi S_{l}^{\text {rest }}$ ) and presence of physical activity (TTS $S_{1}^{e x D}$, TTS $S_{l}^{e x L}$, TT $S_{l}^{e x}$.

$S_{l}^{\text {rest }}$ was lower than $S_{l}^{\text {ex }}\left(12.4 \pm 2.0\right.$ vs. $21.6 \pm 3.710^{-4} \mathrm{dl} / \mathrm{kg} / \mathrm{min}$ per $\mu \mathrm{U} / \mathrm{ml}$, $\mathrm{p}<0.0005)$. Similarly, TTS $S_{\text {rest }}$ was lower than TTS $S_{\text {l }}$ ( $13.9 \pm 2.3$ vs. $23.4 \pm 3.710^{-4}$ $\mathrm{dl} / \mathrm{kg} / \mathrm{min}$ per $\mu \mathrm{U} / \mathrm{ml}, \mathrm{p}<0.0005)$. Correlation between indices derived with the two methods was $R=0.97(p<0.0001)$ both at rest and during exercise. Finally, $T T S_{I}^{\text {restl }}$ was not different from TTS I $^{\text {exL }}\left(2.5 \pm 0.5\right.$ vs. $3.0 \pm 1.110^{-4} \mathrm{dl} / \mathrm{kg} /$ min per $\mu \mathrm{U} / \mathrm{ml}, \mathrm{p}=0.38)$, while TTS $S_{l}^{\text {restt }}$ was lower than TTS $S_{l}^{e \times D}(11.4 \pm 2.0$ vs. $20.4 \pm 3.210^{-4} \mathrm{dl} / \mathrm{kg} / \mathrm{min}$ per $\left.\mu \mathrm{U} / \mathrm{ml}, \mathrm{p}<0.0005\right)$.

In conclusion, we have validated the measurement of insulin sensitivity based on plasma glucose and insulin concentration against the meal triple tracer technique and shown that moderate physical activity almost doubles insulin sensitivity in healthy subjects due to an increased insulin dependent glucose disposal.

Supported by: NIDDK (085516), (DP3094331); FIRB

734-P

Effects of Aerobic Exercise, Resistance Exercise or Both on \% Body Fat in Overweight Adolescents: The HEARTY Trial

RONALD J. SIGAL, ANGELA S. ALBERGA, GARY S. GOLDFIELD, GLEN P. KENNY, STASIA HADJIYANNAKIS, DENIS PRUD'HOMME, JANINE MALCOLM, PENNY PHILLIPS, JINHUI MA, HEATHER TULLOCH, REJEANNE GOUGEON, GEORGE A. WELLS, Calgary, AB, Canada, Ottawa, ON, Canada, Montreal, OC, Canada

The Healthy Eating, Aerobic and Resistance Training in Youth (HEARTY) trial was designed to evaluate the effects of resistance training, aerobic training, and their combination on body composition and cardiometabolic profile in overweight adolescents.

After a 4-week run-in period, 304 previously sedentary post-pubertal adolescents (age14-18 years, $\mathrm{BMI}>85^{\text {th }}$ percentile for age and sex), were randomized to 22 weeks of: diet + aerobic exercise $(\mathbf{A})$, diet + resistance exercise $(\mathbf{R})$, diet + aerobic + resistance exercise $(\mathbf{A}+\mathbf{R})$, or diet-only control (C). Exercise training was performed in community-based facilities, supervised by personal trainers, 4 times/week. The primary outcome was $\%$ body fat measured by Magnetic Resonance Imaging (MRI). Secondary outcomes included waist circumference, lipids, blood pressure (BP), resting energy expenditure (REE) and glucose tolerance. Primary analyses were intention-to-treat. Per-protocol analyses included only subjects $(n=163$ ) completing at least $70 \%$ of prescribed exercise (i.e. $\geq 2.8$ sessions/week).

There were significant $(p<0.001)$ within-group decreases in $\%$ body fat and waist circumference in all 3 exercise groups but not in $\mathrm{C}$. Absolute decreases in $\%$ body fat were $1.1 \%$ in $A$ ( $p=0.06$ vs. C), $1.6 \%$ in $R(p=0.002$ vs. $C$ ), $1.4 \%$ in $A+R(p=0.007$ vs. $C$ ), and $0.4 \%$ in $C$. Waist circumference decreased 3.1 $\mathrm{cm}$ in $A$ ( $p=0.012$ vs. C), $2.2 \mathrm{~cm}$ in $R$ (NS vs. C), $4.2 \mathrm{~cm}$ in $A+R(p=0.013$ vs. $R$ $p=0.13$ vs. $A, p<0.001$ vs. C) and $0.4 \mathrm{~cm}$ in C. In per-protocol analyses, $\%$ body fat decreased $1.2 \%$ in $A, 1.7 \%$ in $R, 2.4 \%$ in $A+R$ ( $p=0.036$ vs. $A, p<0.001$ vs. $\mathrm{C}$, and $0.3 \%$ in $\mathrm{C}$, and waist circumference decreased $3.7 \mathrm{~cm}$ in $\mathrm{A}, 4.5 \mathrm{~cm}$ in $R, 6.8 \mathrm{~cm}$ in $A+R$ ( $p=0.013$ vs. $R, p<0.001$ vs. $C$ ) and $0.6 \mathrm{~cm}$ in $C$. Changes in lipids, BP, REE and glucose tolerance did not differ among groups.

Therefore, aerobic, resistance and combined exercise each reduced $\%$ body fat and waist circumference significantly. Combined aerobic + resistance exercise was superior to either type of exercise alone when performed regularly.

Supported by: CIHR (MCT-71979) 
Meeting and Maintaining High Physical Activity Levels Mitigates Longitudinal Deterioration of Insulin Sensitivity and Metabolic Profiles in Mexican Americans

ZANGHUA CHEN, MARY HELEN BLACK, MIWA TAKAYANAGI, RICHARD M. WATANABE, THOMAS A. BUCHANAN, ANNY H. XIANG, Los Angeles, CA, Pasadena, $C A$

Few studies have examined associations between changes of physical activity (PA) in a free-living environment and changes in type 2 diabetes (T2D)-related quantitative traits. We examined these associations in participants of BetaGene, a study of insulin resistance and beta-cell dysfunction in Mexican Americans ( $n=329)$, with longitudinal anthropometric and metabolic measures over a median follow-up of 4.1 yrs (range 2.7-8.8 yrs). Subjects had mean \pm SD age of $35 \pm 8$ years and BMI of $29.3 \pm 5.9 \mathrm{~kg} /$ $\mathrm{m} 2 ; 72 \%$ were female. Phenotyping included self-reported PA, dual-energy $\mathrm{X}$-ray absorptiometry (DXA) for body fat percentage (\%BF), oral (OGTT), and intravenous (FSIGT) glucose tolerance tests at both baseline and follow-up. Subjects were categorized into two groups. Group $1(n=78)$ did not meet DHHS recommended PA levels (vigorous $\geq 75$ mins/wk or moderate $\geq 150$ mins/ wk) at follow-up. Group 2 ( $n=251$ ) met PA levels, either by maintaining them from baseline onward or attaining them during follow-up. After adjustment for baseline age and sex, group 2 had significantly lower rates of decline in insulin sensitivity (SI), lower rates of increase in 2-hr glucose, fasting and 2-hr insulin, delta 30-min insulin, acute insulin response to glucose (AIR) than group 1 (all $p<0.042$ ). The adjusted mean rates of change for group 2 vs. group 1 were -0.12 vs. -0.21 units/yr for $S I$ ( $p=0.027$ ), 0.10 vs. 0.26 units/yr for 2 -hr glucose $(p=0.008)$. Additional adjustment for baseline and change in $\% B F$ attenuated the differences by $4-26 \%$, but the significant betweengroup differences for SI, 2-hr glucose, fasting insulin, and AIR remained (all $\mathrm{p}<0.047$ ). Our results indicate that attaining and/or maintaining the DHHSrecommended PA level in a free-living environment mitigates the worsening insulin resistance and associated deterioration in metabolic profile that leads to T2D.

Supported by: NIH/NIDDK (5R01DK-61628)

736-P

Exercise Training in the Metabolic Syndrome: Responders are Identifiable by the Pre-Training Fasting Serum Glucose

CHARLES A. STUART, MICHELLE L. LEE, MARK A. SOUTH, MARY E. HOWELL, MICHAEL W. RAMSEY, MICHAEL H. STONE, Johnson City, TN

Combined exercise and weight-reduction programs have been effective in decreasing the insulin resistance of obesity and type 2 diabetes, but the cellular mechanisms of this enhanced insulin action are unclear. We have examined the effect of eight weeks of endurance or strength training without weight loss on insulin resistance in 30 non-diabetic persons with the metabolic syndrome. To our surprise, only about half of the subjects (14 of 30) improved their insulin responsiveness ("responders") to either form of exercise training, as quantified by euglycemic insulin clamps. This result was in spite of improved strength and endurance in both responders and non-responders. In pre-training muscle, pathways of insulin action (insulin receptor, IRS-1, and GLUT4 expression), mitochondrial markers, and muscle fiber diameter were similar between the responders and non-responders. However, fasting serum glucose was higher in non-responders $(108 \pm 3 \mathrm{mg} / \mathrm{dL}$ vs $96 \pm 2, p<0.001)$. The change in insulin responsiveness inversely correlated with the baseline fasting serum glucose. Before training, the non-responders were not more obese, older, nor more insulin resistant. However, the initial muscle fiber composition was different. Type 1 (slow twitch) content was $26 \%$ lower and type 2a (mixed slow and fast twitch myosin) proportion was $25 \%$ higher in responders. Only the responders increased muscle fiber size after training. These data suggest that mild hyperglycemia (impaired fasting glucose) is related to the inadequate response to exercise training without weight loss. Further, hypertrophy of muscle fibers was necessary for insulin responsiveness to improve after exercise training. We conclude that the modestly impaired fasting glucose concentrations in many subjects was a manifestation of a metabolic derangement that caused dysfunction of muscle remodeling pathways and impaired muscle fiber hypertrophy, preventing any improvement in whole body insulin responsiveness.
Effects of Exercise Amount and Intensity versus a Lifestyle Intervention on Fasting Glucose in Adults With Pre-diabetesSTRRIDE-PD

LESLIE H. WILLIS, CRIS A. SLENTZ, LORI A. BATEMAN, ESTHER GRANVIILLE, LUCY W. PINER, CONNIE W. BALES, WILLIAM E. KRAUS, Durham, NC

The Diabetes Prevention Program (DPP) demonstrated that a lifestyle program of diet plus exercise could reduce fasting glucose in pre-diabetics. However, the optimal exercise-only intervention for improving fasting glucose remains unknown. Therefore, we compared three six-month exercise-only groups differing in amount and/or intensity to a lifestyle group of diet and exercise similar to the first 6 months of DPP. Adult (4575 y) participants with pre-diabetes were randomized to: 1) Low-Amount/ Moderate-Intensity exercise (10 Kcal/Kg/wk at $50 \%$ peak V02); 2) HighAmount/Moderate-Intensity exercise $(16 \mathrm{Kcal} / \mathrm{Kg} / \mathrm{wk}$ at $50 \%$ peak V02); 3) High-Amount/Vigorous-Intensity (16 Kcal/Kg/wk at $75 \%$ peak V02); and 4) Diet + Exercise - 7\% weight loss and Low-Amount/Moderate-Intensity exercise (same as group 1). Fasting plasma glucose (FPG) was measured pre and post-intervention, with 127 participants having complete FPG data at both time-points ( 30 per group). The lifestyle diet + exercise group significantly reduced FPG by $5.4 \mathrm{mg} / \mathrm{dL}(\mathrm{p}<0.01)$. However, none of the 3 exercise-only groups significantly changed FPG from the baseline value (LM $-0.2 \mathrm{mg} / \mathrm{dL}$, HM $-1.6 \mathrm{mg} / \mathrm{dL}, \mathrm{HV} 1.9 \mathrm{mg} / \mathrm{dL})$. Surprisingly, even with a significantly higher time commitment and a $60 \%$ increase in caloric expenditure, the High Amount/Moderate intensity group only achieved $28 \%$ of the total effect in FPG compared to diet + exercise. While the mechanisms of how pancreas and liver function reduce FPG are not well understood, these results would suggest that exercise alone may not affect the mechanisms responsible for improving FPG. It is apparent that the diet and induced weight loss component of the DPP lifestyle group is responsible for FPG reduction.

Supported by: 1R01DK081559

738-P

Impact of Adjustable Sit-Stand Workstations on Physical Activity in Sedentary Office Workers

NIRJHAR DUTTA, GABRIEL KOEPP, CARRIE SCHMITZ, STEVEN D. STOVITZ, JAMES

A. LEVINE, MARK A. PEREIRA, Minneapolis, MN, Rochester, MN, Eagan, MN

Excess sedentary time (e.g. sitting) may have health effects such as excess weight gain and diabetes risk. The purpose of this study was to determine whether light non-exercise activity could be increased during the workday by the use of sit-stand workstations. We conducted a randomized cross-over trial in 28 sedentary office workers who participated in a four week intervention period (use of a sit-stand desk with a goal of replacing $50 \%$ of sitting time at work with standing) and a control period (usual sitting work) separated by a two week wash-out period. Based on objective accelerometry as well as questionnaires, subjects replaced between 25\% to $50 \%$ of their sitting time with standing ( $p$-value $<.0001$ ). The intervention also had an effect, assessed by accelerometry, of increasing activity during work hours; for the average 5-day work-week, sedentary time at work was reduced by 3.2 hours and replaced with light physical activity (p-value $<.0001)$. The intervention increased overall sense of well-being, decreased fatigue, and, interestingly, reduced appetite and energy intake. 89\% of the subjects requested to have the workstations permanently installed at their workspace at the end of the study. Overall, a sit-stand workstation appears to be a promising tool to reduce sedentary time in office workers who typically spend most of their workday sitting.

Supported by: University of Minnesota; Mayo Clinic

$\Delta$ 739-P "Dance Dance Revolution" Exergaming vs. Treadmill Exercise in Type 2 Diabetes

STANLEY H. HSIA, LYNNE A. MAGLIANO, HUMBERTO SANCHEZ, THOMAS W. STORER, Los Angeles, CA

Interactive video games that require physical movement may have metabolic benefits. We compared the exercise video game "Dance Dance Revolution" (DDR) to treadmill exercise training (TM) among 76 adult, racial minority, non-insulin-treated type 2 diabetic patients. Subjects with hemoglobin $\mathrm{A}_{1 \mathrm{c}}\left(\mathrm{HbA}_{1 \mathrm{c}}\right)$ between $7.0-10.0 \%$ were randomized to 90 days of $\mathrm{ad}$ libitum access to either DDR or TM at a supervised facility, participating at self-selected frequency, duration and intensity while maintaining usual daily activities. Glycemic control, body composition, and aerobic capacity (via incremental TM exercise with indirect calorimetry and a constant work rate (CWR) endurance test at $80 \%$ of $\mathrm{VO}_{2 \text { peak }}$ ) were compared between baseline 


\section{EXERCISE-HUMAN}

and day-90, adjusting for calorie intake and exercise energy expenditure (EE). All baseline measures were comparable between groups. $\mathrm{HbA}_{1 \mathrm{c}}$ did not change significantly within or between groups (TM: $8.2 \pm 0.9$ to $8.3 \pm 1.5 \%$, $\mathrm{p}=0.58 ;$ DDR: $8.1 \pm 0.9$ to $8.1 \pm 1.2 \%, \mathrm{p}=0.62)$. TM improved VO $\mathrm{O}_{2 \text { eak }}(23.4 \pm 5.6$ to $24.3 \pm 5.7 \mathrm{~mL} / \mathrm{kg} / \mathrm{min}, \mathrm{p}=0.03)$; increased CWR endurance time $(3.9 \pm 1.6$ to $6.8 \pm 4.8 \mathrm{~min}, \mathrm{p}<0.01)$ and decreased isotime heart rate ( $\mathrm{HR}$, at the same time as the baseline endurance test termination, $162 \pm 19$ to $156 \pm 20 \mathrm{~min}^{-1}$, adjusted $p \leq 0.02$ ) compared to DDR; and lowered relative fat mass (36.2 \pm 8.2 to $35.8 \pm 7.9 \%, p=0.02)$ and increased relative lean mass $(61.3 \pm 7.9$ to $61.7 \pm 7.6 \%, p=0.01)$. Body composition and aerobic capacity did not change significantly with DDR. Exercise HR recordings and estimated EE revealed higher exercise frequency for TM vs. DDR in the first month, and significant declines in duration, frequency and EE over time in both groups, but a faster decline in exercise frequency with TM than DDR $(p<0.01)$. We conclude that although ad libitum participation in DDR does not decline in the short term as rapidly as TM among adult minority diabetic patients, it also does not promote the same improvements in body composition and aerobic capacity as TM, and does not lead to better glycemic control.

Supported by: NIH/NIMHD (U54MD007598)

740-P

Autonomous use of the Exercise Game Wii Fit Plus Improves Glucometabolic Control and Quality of Life in Type 2 Diabetes Patients: A Randomized Controlled Trial

KERSTIN KEMPF, STEPHAN MARTIN, Düsseldorf, Germany

Lifestyle intervention in type 2 diabetes mellitus (T2DM) is effective but needs a special local setting and is costly. Therefore, we tested in a randomized-controlled trial the hypothesis that the autonomous use of the exercise game Wii Fit Plus over 12 weeks leads to a healthier lifestyle and better quality of life in T2DM patients.

Participants $(n=220)$ were randomized into two groups. The intervention group got a Wii console, a balance board and the exercise game Wii Fit Plus for 12 weeks. The control group remained in routine care and received the intervention 12 weeks later. At baseline and after 12 weeks (and for the control group also after 12 weeks of intervention) health parameters were measured by the treating physician and medication, physical activity and quality of life (using validated self-assessment questionnaires PAID, SF-12, WHO-5, CES-D) was assessed. Wilcoxon signed rank test was used to test if differences were different from 0 . Inter-group differences were tested by Mann Whitney test.

$80 \%$ of participants completed the 12-week study. Patients in the intervention group significantly reduced $\mathrm{HbA} 1 \mathrm{c}(0.3 \pm 1.1 \%$; $p<0.001)$, fasting blood glucose $(7.7 \pm 35.0 \mathrm{mg} / \mathrm{dl} ; \mathrm{p}=0.04)$, weight $(1.2 \pm 4.7 \mathrm{~kg} ; p<0.001)$ and body mass index $(0.4 \pm 1.6 \mathrm{~kg} / \mathrm{m} 2 ; p<0.001)$ in comparison to the control group. Daily physical activity increased significantly $(p<0.001)$. Diabetes-dependent impairment $(p<0.001)$, mental health $(p=0.02)$, subjective wellbeing $(p<0.01)$ and quality of life $(p=0.03)$ also improved, and the number of depressed patients decreased $(p=0.02)$. Similar improvements also occurred in the control group after intervention.

In this proof-of-principle trial a low-threshold intervention with the exercise game Wii Fit Plus was able to motivate T2DM patients to improve physical activity, glucometabolic control and quality of life. Exercise games may potentially be used in a home setting to reduce sedentary behavior in T2DM.

Supported by: Novartis Pharma GmbH; Foundation Motivation zur LebensstilÄnderung

741-P

Endurance Exercise Enhances Insulin Sensitivity by Increasing Adiponectin Multimer Distribution in Obese Young Males

YU SIK KIM, SHINAE KANG, DONG WOOK YEO, SOON AE KIM, SUN HEE BEOM, DA WOON HAN, JONG SUK PARK, KYUNG RAE KIM, SANG HOON SUH, CHUL WOO AHN, SOHEE KIM, Seoul, Republic of Korea

Adiponectin, a hormone synthesized by adipocytes, modulates a number of metabolic processes, including glucose homeostasis. In circulation, adiponectin molecules automatically self-associate to form oligomeric isoforms- high molecular (HMW)-; middle molecular (MMW)-; and low molecular (LMW) adiponectin. Among these, HMW is known as the most biologically active in glucose control. As endurance exercise is important prevention and treatment modality for insulin resistance (IR), this study is designed to examine the influence of endurance exercise on IR, total adiponectin and multimer distribution in obese males.

39 obese, normoglycemic young Korean males were randomly assigned to control $(C, n=10)$ or exercise group $(E x, n=29)$. The subjects in Ex underwent a 8-week -endurance exercise (2400Kcal/week). BMI, \% body fat, waist circumference (WC), fasting plasma insulin (FPI) and glucose (FPG), total adiponectin and multimer distributions were measured. BMI, WC, \% body fat, $\mathrm{FPI}$, and HOMA-IR were significantly decreased in $E x(p<0.01)$. Unexpectedly, total adiponectin decreased significantly in $E x(p<0.01)$, however, HMW distribution increased from $2.27 \pm 0.55$ to $15.48 \pm 8.47(p<0.01)$. This increase was significantly associated with enhanced HOMA-IR $(r=0.387, p=0.015)$, and when adjusted for $\mathrm{BMI}, \mathrm{WC}$ and $\%$ body fat $(r=0.357, p=0.027$. These suggest that endurance exercise alleviated IR by increasing HMW.

Our study highlights the beneficial influence of endurance exercise on IR. It is noteworthy that the alteration in adiponectin multimer distributions with endurance exercise indicates a potential source for metabolic disorders in obese individuals. This study was limited to the normoglycemic obese individuals; further study with hyperglycemic individuals is warranted. Insulin Degludec and Insulin Glargine

SIMON R. HELLER, STEPHEN C.L. GOUGH, DAVID S. OYER, KLAUS H. JENSEN, ONA KINDURYTE, ATHENA PHILIS-TSIMIKAS, Sheffield, United Kingdom, Oxford, United Kingdom, Chicago, IL Søborg. Denmark, Bagsvaerd, Denmark, La Jolla, CA

During exercise, increased glucose requirements as well as increased insulin sensitivity can lead to hypoglycemia in patients with diabetes. Exercise intensity, dose timing and the individual pharmacodynamic properties of insulin can affect the risk of hypoglycemia. Insulin degludec (IDeg) is a new basal insulin with an ultra-long and stable glucose-lowering effect.

We investigated exercise-related hypoglycemia in 7 randomized, openlabel, treat-to-target, clinical trials with IDeg vs. insulin glargine (IGlar) once daily. Trials were of 26 or 52 weeks in type 1 (T1D; N=1116) and type 2 diabetes (T2D; N=3598). Patients reported hypoglycemic episodes in their subject diaries and were instructed to note any relation to exercise (based on their own judgment). Confirmed hypoglycemia was defined as $\mathrm{PG}<56 \mathrm{mg} / \mathrm{dL}$ (3.1 $\mathrm{mmol} / \mathrm{L}$ ) or severe episodes requiring assistance. Nocturnal hypoglycemia was defined as confirmed episodes occurring between 00:01 and 05:59

The proportion of patients experiencing $\geq 1$ confirmed hypoglycemic episode related to exercise was similar with IDeg and IGlar for T1D and T2D (table). Few exercise-related nocturnal confirmed hypoglycemic episodes were reported, and the proportions were similar for IDeg and IGlar. In both arms, more exercise-related hypoglycemia occurred in patients with T1D than with T2D. In conclusion, the data suggest no increased risk of selfreported exercise-related hypoglycemia with IDeg compared to IGlar.

\begin{tabular}{|c|c|c|c|}
\hline & T1D & $\begin{array}{l}\text { T2D basal- } \\
\text { bolus } \\
\text { therapy }\end{array}$ & $\begin{array}{l}\text { T2D basal-oral } \\
\text { therapy }\end{array}$ \\
\hline & IDeg IGlar & IDeg IGlar & IDeg IGlar \\
\hline Total N subjects & $801 \quad 315$ & $753 \quad 251$ & $1734 \quad 860$ \\
\hline $\begin{array}{l}\text { Total N subjects with confirmed } \\
\text { hypoglycemic episodes }\end{array}$ & 769303 & 609206 & 779380 \\
\hline $\begin{array}{l}\text { Total } N \text { subjects with } \geq 1 \text { exercise-related } \\
\text { confirmed hypoglycemic episode (\% of } N \\
\text { with confirmed hypoglycemic episodes) }\end{array}$ & $\begin{array}{cc}614 & 242 \\
(80 \%) & (80 \%) \\
\end{array}$ & $\begin{array}{r}311 \quad 106 \\
(51 \%)(51 \% \\
\end{array}$ & $\begin{array}{cc}163 & 83 \\
(21 \%) & (22 \%) \\
\end{array}$ \\
\hline \multicolumn{4}{|c|}{$\begin{array}{l}\text { Trials: T1D: NN1250-3770, NN1250-3583 (basal-bolus therapy), T2D: } \\
\text { NN1250-3586, NN1250-3668, NN1250-3672, NN1250-3579 (all basal } \pm \text { oral), } \\
\text { NN1250-3582 (basal-bolus therapy). Safety analysis set: subjects exposed to } \\
\geq 1 \text { dose of trial product. }\end{array}$} \\
\hline Supported by: Novo Nordisk, Inc. & & & \\
\hline
\end{tabular}

743-P

Varying Durations and Intensities of Exercise and Blood Glucose Responses

SHERI R. COLBERG, MANUEL J. HERNANDEZ, FATIMA G. SHAHZAD, Norfolk, VA, Berkeley, CA

During the 2012 Big Blue Test (run by the Diabetes Hands Foundation to raise awareness of the impact of exercise on diabetes control), participants anonymously entered their diabetes status, type of exercise done, intensity (moderate or vigorous), duration (10 or less, 11 to 19,20 to 29 , or 30 or more $\mathrm{min})$, time elapsed since most recent meal, and blood glucose (BG) readings before and after the activity at www.bigbluetest.org. A total of 7,157 exercise-associated BG pairs (before/after) from persons with diabetes were included. Walking was the most frequent activity $(48.5 \%)$, followed by other (non-specified) exercise $(18.7 \%)$, running/jogging $(11.9 \%)$, cycling $(8.8 \%)$, conditioning machines $(6.4 \%)$, and dancing $(5.7 \%)$. Overall, mean BG levels were $31.3 \mathrm{mg} / \mathrm{dL}$ lower $( \pm 47.1)$ following any exercise, a decrease of $16.8 \%$. 


\section{NUTRITION-CLINICAL}

Longer exercise times generally resulted in increasingly greater decreases in $B G$ for moderate exercise $(p<0.001)$, although results were mixed for vigorous (table). The largest decrease in BG occurred following 30 or more min of exercise undertaken $1 \mathrm{hr}(-49.3 \pm 53.1 \mathrm{mg} / \mathrm{dL})$ or $2 \mathrm{hr}(-46.4 \pm 49.8)$ after the last meal ( $\mathrm{p}<0.001)$. Overall, $75.8 \%$ of participants experienced a decrease in BG, $8.8 \%$ were unchanged $( \pm 5 \mathrm{mg} / \mathrm{dL})$, and $15.4 \%$ had an increase. Thus, this year's Big Blue Test confirms that participation in varying types, intensities, and durations of exercise generally lowers BG in most individuals, although exercise of longer duration is likely most effective.

Mean Change in Blood Glucose (mg/dL) by Exercise Intensity and Duration

\begin{tabular}{lllll} 
& 10 Min or Less & 11 to 19 Min & 20 to 29 Min & 30 Min or More \\
\hline Moderate, $n=5,051$ & $-16.9 \pm 43.8$ & $-25.6 \pm 32.9$ & $-29.9 \pm 40.0^{*}$ & $-39.0 \pm 48.6^{* \infty \dagger}$
\end{tabular}

\begin{tabular}{lllll}
\hline Vigorous, $n=2,106$ & $-25.1 \pm 51.4$ & $-22.1 \pm 41.8^{\ddagger}$ & $-36.1 \pm 49.9 * \infty *$ & $-27.6 \pm 55.5^{* \neq}$ \\
\hline
\end{tabular}

$p<0.001$ vs. 10 Min or Less Moderate, ${ }^{\infty} p<0.001$ vs. 11 to 19 Min Moderate, ${ }^{\dagger} \mathrm{p}<0.001$ vs. 20 to 29 Min Moderate; ${ }^{\ddagger} \mathrm{p}<0.001$ vs. 30 Min or More Moderate; ${ }^{*} \mathrm{p}<0.001$ vs. 11 to 19 Min Vigorous; all values are mean \pm SD

\section{Supported by: Roche Diabetes Care}

744-P

Differential Effects of Antecedent GABA (A) Activation on Counterregulatory Responses to Exercise in Type 1 Diabetic and Nondiabetic Men

MAKA HEDRINGTON, DONNA B. TATE, MAIA MIKELADZE, NINO G. JOY, STEPHEN N. DAVIS, Baltimore, MD

Previous studies have demonstrated that antecedent activation of GABA (A) receptors with the benzodiazepine alprazolam (Alp) can blunt counterregulatory responses (CR) during subsequent exercise as compared to placebo (P). The aim of this study was to determine whether antecedent stimulation of $\mathrm{GABA}(\mathrm{A})$ receptors could have differential effects in type 1 diabetic (T1DM) and non diabetic (ND) individuals. 30 (16M/14F) T1DM (HBA1c $7.5 \pm 0.3 \%$ ) and $31(16 \mathrm{M} / 15 \mathrm{~F}$ ) matched ND (age $29 \pm 4$ years, BMI $25 \pm 2 \mathrm{~kg} / \mathrm{m} 2$, V02max $35 \pm 2 \mathrm{ml} . \mathrm{kg}-1$.min-1) subjects were studied during single blind, randomized, 2 day protocols. Day 1 consisted of morning and afternoon $2 \mathrm{hr}$ euglycemic clamps with $1 \mathrm{mg}$ Alp or $\mathrm{P}$ given 30 min before each clamp. Day 2 consisted of 90 min euglycemic cycling exercise at $50 \%$ V02 max. Day 2 blood glucose levels $(5.3 \pm 0.02 \mathrm{mmol} / \mathrm{l})$ were similar in all groups. Changes in insulin concentration $(\Delta 1.8 \pm 1 \mathrm{pmol} / \mathrm{l})$ were also similar in all groups. CR during exercise were reduced $(p<0.05)$ following GABA $(A)$ activation in both, T1DM and ND subjects. However, there were greater $(p<0.05)$ blunting effects of GABA $(A)$ activation on autonomic nervous system (ANS) (epinephrine, norepinephrine), neuroendocrine (cortisol) and metabolic (lactate) responses in ND as compared to T1DM (Table 1). We conclude that key ANS and neuroendocrine responses in T1DM are more resistant to the blunting effects of GABA (A) as compared to ND man.

Table 1. Change from baseline to final 15 minutes of exercise

\begin{tabular}{lcccc}
\hline & ND+Alp & ND $+\mathrm{P}$ & T1DM+Alp & T1DM+P \\
\hline$\Delta$ Epinephrine $(\mathrm{pmol} / \mathrm{l})$ & $144.3 \pm 6.7^{*^{\prime \prime}}$ & $768.9 \pm 213.6$ & $299.0 \pm 48.1^{\wedge 1^{* \prime}}$ & $581.7 \pm 97.1$ \\
\hline$\Delta$ Norepinephrine $(\mathrm{nmol} / \mathrm{l})$ & $1.1 \pm 0.3^{* \prime}$ & $5.0 \pm 0.6$ & $2.2 \pm 0.3^{\text {}^{* \prime}}$ & $3.9 \pm 0.5$ \\
\hline$\Delta$ Glucagon $(\mathrm{ng} / \mathrm{l})$ & $2.5 \pm 2.1^{* \prime}$ & $12.2 \pm 1.7$ & $5.2 \pm 1.3^{* \prime}$ & $9.3 \pm 1.6$ \\
\hline$\Delta$ Cortisol $(\mathrm{nmol} / \mathrm{l})$ & $-76.1 \pm 36.8^{* \prime}$ & $181.1 \pm 53.2$ & $56.8 \pm 35.4^{\wedge * \prime}$ & $204.6 \pm 60.2$ \\
\hline$\Delta$ Growth Hormone $(\mathrm{ng} / \mathrm{ml})$ & $0.7 \pm 1.7^{* \prime}$ & $10.4 \pm 2.5$ & $4.3 \pm 1.4^{* \prime}$ & $9.8 \pm 1.5$ \\
\hline$\Delta$ Lactate $(\mathrm{mmol} / \mathrm{l})$ & $0.2 \pm 0.1^{* \prime}$ & $0.7 \pm 0.2$ & $0.8 \pm 0.2^{\wedge}$ & $1.3 \pm 0.1$ \\
\hline
\end{tabular}

${ }^{*} \mathrm{p}<0.05$ compared to ND+P

$p<0.05$ compared to T1DM+P

$\wedge p<0.05$ compared to ND+Alp

Supported by: NIH (5P01HL056693)

745-P

Effects of Exercise Amount and Intensity versus Lifestyle Intervention on Metabolic Syndrome in Adults With Prediabetes-STRRIDE PD

LORI A. BATEMAN, CRIS A. SLENTZ, LESLIE H. WILLIS, ESTHER GRANVILLE, LUCY W. PINER, CONNIE A. BALES, WILLIAM E. KRAUS, Durham, NC

While aerobic exercise improves metabolic syndrome (MetSyn) risk factors, it is not known whether a certain amount and/or intensity of exercise can yield a similar improvement as a combined exercise and diet lifestyle program. We compared three 6-month aerobic exercise groups differing in amount and/or intensity to an exercise + diet group similar to the Diabetes Prevention Program. Sedentary, overweight adults (45-75 yrs) with prediabetes (fasting plasma glucose 95-125) were randomized

For author disclosure information, see page 829 . to: 1) Low-Amount/Moderate-Intensity $\left(10 \mathrm{kcal} / \mathrm{kg} / \mathrm{wk}\right.$ at $50 \%$ peak $\left.\mathrm{VO}_{2}\right)$; 2) High-Amount/Moderate-Intensity (16 kcal/kg/wk at $50 \%$ peak $\left.\mathrm{VO}_{2}\right) ; 3$ ) High-Amount/Vigorous-Intensity ( $16 \mathrm{kcal} / \mathrm{kg} / \mathrm{wk}$ at $75 \%$ peak $\left.\mathrm{VO}_{2}\right)$; or 4$)$ Low-Amount/Moderate-Intensity + Diet ( $7 \%$ weight loss). We calculated a continuous MetSyn z score on the 112 participants with complete data for all 5 MetSyn criteria. The High-Amt/Mod-Int group had a significant improvement in MetSyn z score. The Low-Amt/Mod-Int + Diet group had a significant improvement in MetSyn $z$ score and was significantly different from all 3 exercise only groups. This data suggest that a High-Amt/Mod-Int exercise program can improve MetSyn risk. However, the findings suggest that a significantly greater effect in improved MetSyn risk is obtained when adding a weight loss diet to the Low-Amt/Mod-Int exercise intervention.

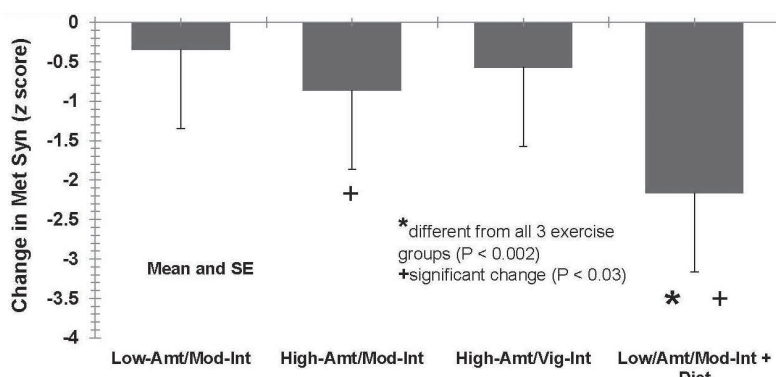

\section{NUTRITION-CLINICAL}

Guided Audio Tour: Effective Dietary Interventions (Posters: 746- $P$ to 753-P), see page 17.

ก 746-P

A 10 Week Internet Lifestyle Intervention for Reducing Metabolic Syndrome (MetS): Intervention Significantly Reduces MetS Risk Compared to Age Matched Wait-List Controls

MARCIA B. UPSON, SCOTT E. CONARD, CANDICE J. BURGESS, CARROLL W. HUGHES, Garland, TX, Dallas, TX

Prediabetes and Metabolic Syndrome [MetS] have been associated with an increased risk of developing diabetes. A ten week, internet course with standardized behavior-based interventions called Natuturally Slim (NS) was administered to 9,370 individuals who met one or more risk factors for MetS. The NS participants were compared to an age-matched (mean age $=46$ for both groups) wait-list control group $(\mathrm{n}=2,779)$. Pre and Post intervention blood values and biometric measures were obtained for all 12,149 subjects. There was a significant change in the NS participants based on pre and post blood values compared to controls (all $p<.0001$ ): NS waist circumference versus wait-list controls $(-2.0$ inches vs. +0.3$)$; triglycerides $(-25 \mathrm{mg} / \mathrm{dl}$ vs. $+0.3)$, blood pressure $(-2.5 \mathrm{~mm} / \mathrm{Hg}$ vs. +0.1$)$, glucose $(-3.1 \mathrm{mg} / \mathrm{dl}$ vs. +0.2$)$. The reductions led to significant resolution of MetS in those having three or more of the MetS criteria for the NS group but not the wait-list control: $33 \%$ reduction of meeting MetS criteria for the NS group compared to an $8 \%$ increase in the wait-list group. Sixty two (61.7) percent of those in the NS MetS group only, had at least one risk factor for MetS resolve after ten weeks, with 44\% no longer meeting MetS criteria at the conclusion of the program. Weight loss occurred in $87 \%$ of the NS participants (9.9 pounds average vs. 0.75 pounds in controls) sustained over two years.

Comparison of NS Lifestyle Intervention to Controls in Reductions of Metabolic Syndrome Risks

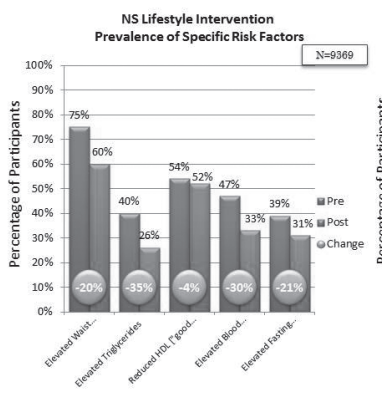

$\Omega$ Guided Audio Tour poster

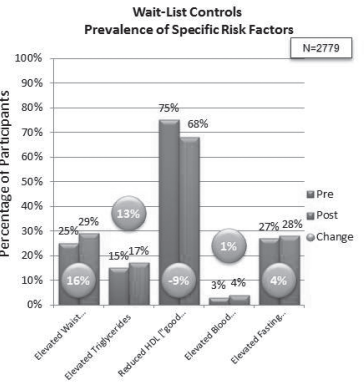

A ADA-Funded Research 
Beneficial Effects of Kimchi on Glucose Metabolism Related Parameters in Subjects With Prediabetes

SO-YEON AN, MIN SUK LEE, JA YOUNG JEON, MIN JEONG LEE, TAE HO KIM, JA YOUNG YOON, CHANG-OK OK, HYE-KYOUNG LEE, WON-SUN HWANG, SUN JUNG CHOE, SEUNG JIN HAN, HAE JIN KIM, DAE JUNG KIM, KWAN-WOO LEE, Suwon, Republic of Korea, Goyang, Republic of Korea

With the increased incidence of diabetes mellitus, the importance of early intervention on prediabetes has been emphasized. We previously reported that fermented Kimchi, a Korean traditional food, reduced body weight and improved metabolic parameters in overweight subjects. We hypothesized that Kimchi and its fermented form would have beneficial effects on glucose metabolism in patients with prediabetes.

A total of 21 subjects with prediabetes were enrolled. During the first 8 weeks, they consumed either fresh or fermented Kimchi. Fresh Kimchi (1-dayold) and fermented Kimchi (10-day-old) were only different in the degree of maturity. After a 4 week washout period, they consumed Kimchi vice versa during the next 8 weeks (crossover design). Anthropometric parameters and blood test results were analyzed.

The amount of fiber intake increased after consumption of fresh or fermented Kimchi, as expected $(p<0.001)$. It is noteworthy that the amount of fat intake decreased after consumption of both types of Kimchi $(p=0.016$ for fresh Kimchi, $p<0.001$ for fermented Kimchi). Among anthropometric parameters, body weight, body mass index, and waist circumference decreased after consumption of both types of Kimchi. Blood pressure (BP) was improved after consumption of fermented Kimchi. While HbA1c, fasting insulin, HOMA-IR, Matsuda index was improved after consumption of any Kimchi, quantitative insulin-sensitivity check index and disposition index showed improvement only after consumption of fermented Kimchi. The percentages of improved glucose tolerance were 9.5\% in the fresh Kimchi group and $23.8 \%$ in the fermented Kimchi group.

Consumption of fresh or fermented Kimchi had beneficial effects on glucose metabolism related parameters as well as anthropometric parameters in subjects with prediabetes. In addition, fermented Kimchi had additional effects on BP and insulin sensitivity. The percentage of improvement in glucose tolerance was high in the fermented Kimchi group.

ก 748-P

The Effects of Resistant Starch on Glycemic Control in Type 2 Diabetes

CAROLINE BODINHAM, LEANNE SMITH, DAVID L. RUSSELL-JONES, A.M. UMPLEBY, M.D. ROBERTSON, Guildford, United Kingdom

Increased dietary fiber intake is linked to beneficial effects in type 2 diabetes; however, the exact mechanism remains unclear. Resistant starch, a non-viscous fiber (high amylase maize; HAM-RS2) has been shown to improve insulin secretion and sensitivity in metabolic syndrome but the role in diabetic control is unknown. We conducted a 12 week crossover intervention study in patients with type 2 diabetes to determine whether $40 \mathrm{~g}$ HAM-RS2 per day added to the diet would improve glycemic control and insulin sensitivity compared to a glycemic carbohydrate matched placebo. Seventeen patients (12M, 5F) with diet/exercise or metformin controlled diabetes (HbA1c $46.6 \pm 2 \mathrm{mmol} / \mathrm{mol}$ ) consumed either the HAMRS2 supplement or the placebo daily for 12 weeks and at the end of each intervention attended for 2 clinical investigations; a 2-step euglycemic-

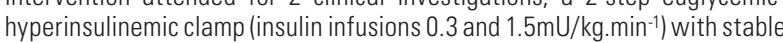
isotopes to differentiate peripheral and hepatic insulin sensitivity and a meal tolerance test with arterio-venous sampling across the forearm muscle as a direct measure of tissue flux. There was no effect of treatment on $\mathrm{HbA1c}$ energy intake or body weight. Increased HAM-RS2 resulted in significantly lower postprandial glucose concentrations $\left(\mathrm{AUC}_{0-120 \mathrm{mins}} 949 \pm 31\right.$ vs $1005 \pm 34$ mmol.min..$^{-1} ; p=0.036$ ) and a trend for higher glucose uptake into the forearm muscle $\left(\mathrm{AUC}_{0-120 \mathrm{mins}} 120 \pm 24\right.$ vs $75 \pm 11 \mathrm{mmol} .100 \mathrm{mg}$ tissue/min..$^{-1} ; p=0.077$ ). However, there was no effect of treatment on insulin sensitivity (peripheral or hepatic). HAM-RS2 treatment lowered fasting non-esterified fatty acid (NEFA) concentrations ( $500 \pm 100$ vs $600 \pm 50 \mu \mathrm{mol} / \mathrm{l}, p=0.004$ ) with greater suppression of NEFA by insulin. Increasing HAM-RS2 has a beneficial effect on postprandial glucose concentrations and the insulin sensitivity of adipose tissue metabolism. However, longer term consumption may be required before beneficial effects on glycemic control and insulin sensitivity are observed in individuals with type 2 diabetes.

Supported by: Diabetes UK
Do Fish Oils Improve Glucose and Protein Metabolism in Human Subjects With Impaired Glucose Regulation?

LOUISE F. CLARK, CAROLE THIVIEGE, SUSAN C. MCGEOCH, PRAKASH ABRAHAM, DONALD W.M. PEARSON, FRANK THIES, GERALD E. LOBLEY, Aberdeen, United Kingdom

The n-3 polyunsaturated fatty acids ( $n-3$ PUFA) have been postulated to improve insulin resistance since the 1960s when a reduced prevalence of type 2 diabetes was observed in Alaskan Inuits consuming a traditional diet. These findings were supported by animal studies but results of human intervention studies have been variable, with most showing no change in glucose metabolism. More recent studies in growing farm animals suggested that muscle membrane phospholipids (PL) required to be enriched to a minimum of $14 \%$ n-3 PUFA for a change in insulin sensitivity to occur.

This study sought to establish the effect of long-term (9 mo) high-dose $(3 \mathrm{~g} / \mathrm{d})$ supplement of $\mathrm{n}-3$ PUFA on insulin sensitivity for glucose and protein metabolism.

Male and female subjects ( $n=33$ ) with impaired glucose regulation underwent hyperinsulinemic-euglycemic-euaminoacidemic clamps preand post-intervention of either n-3 PUFA or a control oil. A second cohort $(n=3)$ who all received $n-3$ PUFA supplementation underwent pre- and postintervention muscle biopsies.

A secondary outcome was to determine whether erythrocyte membrane $\mathrm{PL}$ could act as a surrogate for muscle membrane PL.

In the clamp cohort, there were no changes in glucose metabolism post-intervention; but insulin-stimulated whole body protein metabolism increased $(P=0.003)$ following the fish oil intervention. Fish oil increased erythrocyte $n-3$ PUFA (from 9 to $16 \%$ of total PL) in both the clamp and biopsy cohort but in the latter, muscle membranes only increased from 3 to $8 \%$.

In support of the majority of other reported studies, chronic n-3 PUFA supplementation did not affect glucose metabolism. Nonetheless, the enhanced insulin-stimulated protein metabolism supports a recent finding of increased muscle protein synthesis. These changes in protein metabolism may reduce the sarcopenia associated with aging, potentially delaying the progression of frailty. 


\section{NUTRITION—CLINICAL}

ก 751-P Impaired Fasting Glucose in Youth With Cystic Fibrosis: Transient Decline in Body Mass Index Z-Score

ARTI SHAH, DEBORAH ELDER, MATTHEW FENCHEL, RHONDA SZCZESNIAK, Cincinnati, $\mathrm{OH}$

Body Mass Index z-score (BMIz) is lower in subjects with abnormal glucose tolerance in Cystic Fibrosis (CF). Lower BMlz is a known risk factor for decline in lung function in CF. However, a recent study reported that Impaired Fasting Glucose (IFG, Fasting Glucose 100-125 mg/dl) appeared to improve survival and was not associated with a worse nutritional or pulmonary status. To confirm this unexpected finding, we performed a retrospective chart review of subjects with CF $(n=174)$ between ages 6 to 18 years from January 1 , 2004 to December 31, 2011 at Cincinnati Children's Hospital Medical Center (CCHMC). The baseline characteristics including age, gender, race, CF mutation, BMlz and forced expiratory volume in 1 second percentile (FEV1\%) were established for subjects with Normal Glucose Tolerance (NGT) and IFG. We then used an analysis of covariance (ANCOVA) to assess the change in BMIz and FEV1\%, 2 and 4 years from baseline in NGT and IFG. Similar to previous reports, $56 \%(97 / 174)$ had NGT and $14 \%(24 / 174)$ had IFG. The remaining subjects were excluded due to other forms glucose intolerance. There was no difference in baseline characteristics of age, gender, race, CF mutation, BMIz and FEV1\% between the NGT and IFG group. When compared to NGT, IFG was associated with a significant decline of BMIz at 2 years $(p<0.05)$, but not 4 years. Absolute FEV1\% declined in IFG and NGT groups, though the difference between the two groups was not statistically significant. We conclude, in contrast to the previous report, IFG is associated with a transient decline in $\mathrm{BMIz}$ at 2 years when compared to NGT group. We suspect that prompt nutritional intervention resulted in the improved nutritional status at 4 years. Further study of IFG is required to more completely define the risk profile of IFG in CF.

\section{2-P}

Effects of 16 Week Vitamin D Supplementation in Hispanic Americans With Type 2 Diabetes Mellitus

SARA E. MAHONEY, CHRISTOPHER HEARON, Kingsville, TX

Although low vitamin D status is associated with increased fasting blood glucose and increased insulin resistance in diabetics, evidence for the efficacy vitamin $D$ supplementation in reducing these markers has been variable. One limitation of these studies is that they primarily examine only Caucasian or African-American diabetic populations. The present study examined the effects of 4,000 IU per day of vitamin D (cholecalciferol) for 16 weeks on diabetic Hispanics. Sixteen Hispanic subjects diagnosed with T2DM were recruited. Subjects were randomized into two groups ( $n=8 /$ group): vitamin D (D), which was given 4,000 IU of cholecalciferol daily, and control (C), which was given a placebo daily. Both groups reported to the laboratory every 4 weeks to undergo a battery of tests, which included dietary recall, a sun exposure questionnaire, and anthropometric measures. Blood was drawn at 0, 8 and 16 weeks to assess fasting serum glucose, insulin, and 25(OH)D. Insulin resistance was calculated by using HOMA-IR. Statistical comparisons were made using a two-way, repeated measures ANOVA. Subjects were overweight (BMI $\left.35.6 \pm 6.3 \mathrm{~kg} \cdot \mathrm{m}^{-2}\right)$, had prehypertension $(132.2 / 87.4 \pm 8.8 / 9.3 \mathrm{mmHg})$, and elevated waist to hip ratio $(0.91 \pm 0.05)$. At baseline, subjects were vitamin $D$ insufficient, with no significant differences

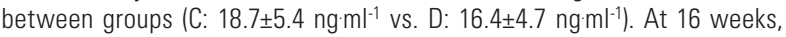
vitamin $D$ was significantly increased in the $D$ group to $48.2 \pm 8.9 \mathrm{ng}^{-\mathrm{ml}^{-1}}$ ( $p$ $<0.05)$. Following supplementation, there was a modest but significant $(p=$ 0.047 ) decrease in fasting blood glucose in the $D$ group $\left(169.1 \pm 10.3 \mathrm{~g} \cdot \mathrm{dL}^{-1}\right)$ as compared to baseline $\left(187.7 \pm 9.8 \mathrm{~g}_{\mathrm{dL}} \mathrm{L}^{-1}\right)$. Insulin resistance, however, did not significantly improve. Finally, there was no significant change in the $D$ group following supplementation from baseline for any anthropometric measures. This pilot study demonstrates a modest, but significant effect of vitamin D supplementation on fasting blood glucose in diabetic Hispanics.

\section{ก 753-P}

Dietary Fiber Intake and Inflammation in Type 1 Diabetes Subjects FERNANDA BERNAUD, Porto Alegre, Brazil

Higher intake of dietary fiber is associated with lower risk of coronary heart disease (CHD), the leading cause of mortality among people with type 1 diabetes (T1D). Population-based studies have shown an inverse association between nutrition and $\mathrm{C}$-reactive protein (CRP). Few studies report the nutrition in individuals with T1D.

We aimed to ascertain the association between fiber intake and hs-CPR levels. This cross-sectional study was conducted with 106 outpatients with
T1D; age $40 \pm 11$ years; diabetes duration of $18 \pm 8.8$ years. Dietary intake was evaluated by 3 -day weighed-diet records. Patients were categorized in 2 groups, according fiber intake ( $>20 \mathrm{~g} /$ day and $<20 \mathrm{~g} /$ day). The group with fiber intake $>20 \mathrm{~g} /$ day had lower hs-CRP levels [median $\left(25^{\text {th }}-75^{\text {th }}\right), 1.9 \mathrm{mg} / \mathrm{dl}(1.0-$ 4.4) vs. $0.7 \mathrm{mg} / \mathrm{dl}(0.4-2.4) ; P=0.002]$, than the other group. Controlled for $\mathrm{HbA1c}$ and energy intake, an inverse relation was observed between hs-CRP level and total fiber $[\beta=-0.030$ (SE: 0.0120), $P=0.02$ ], soluble fiber $[\beta=$ - 0.078 (SE: 0.0421), $P=0.06]$ and insoluble fiber $[\beta=-0.039$ (SE: 0.01761), $\mathrm{P}=0.026]$. Even, after adjustment for $\mathrm{HbA1c}$ and protein or carbohydrates intake, fibers remained associated with lower hs-CRP levels. Total fibers were stratified in 4 groups: < $10 \mathrm{~g} /$ day, from 10 to $20 \mathrm{~g} /$ day, from 20 to $30 \mathrm{~g} /$ day and $>30 \mathrm{~g} /$ day; adjusted for HbA1c stratified in tertiles. Compared to the group who ingested $<10 \mathrm{~g} /$ day of total fiber (referent group), the group who consumed $>30 \mathrm{~g}$ had significantly lower hs-CRP levels [-2.45 mg/L, P=0.012] independent of the $\mathrm{HbA1c}$ values. Also, in comparison to the lowest tertile of HbA1c (the referent group), the subjects in the highest tertile had higher hs-CRP levels $[1.387 \mathrm{mg} / \mathrm{L}, \mathrm{P}=0.006]$, independent of the fiber intake. The present study suggests that an increased consumption of dietary fiber > $30 \mathrm{~g} /$ day, may play a role in reducing inflammation and, thus the risk of $\mathrm{CHD}$ in individuals with T1D.

Supported by: FIPE
754-P

\section{WITHDRAWN}

755-P

Impact of a Nutrition Educational Intervention Coupled With Improved Access to Fresh Produce on Fruit and Vegetable Purchase and Consumption in Patients With Type 2 Diabetes

ELEANOR M. WEINSTEIN, RODOLFO J. GALINDO, MARTIN FRIED, LISA RUCKER, NICHOLA J. DAVIS, Bronx, NY

The Bronx borough of New York City has high rates of obesity and diabetes. Increased consumption of fruit and vegetables (F\&V) is associated with lower rates of obesity and diabetes. The purpose of this study was to test the impact of distributing coupons, redeemable at farmer's markets, and an educational intervention on F\&V purchase and consumption. Seventyeight participants, recruited from a primary care clinic in the Bronx, were randomized to receive the standard of care or a one hour educational session focused on benefits of F\&V consumption and $\$ 6$ in coupons. Questionnaires assessed demographics, F\&V intake and farmer's market purchasing at baseline and 12 weeks. Unpaired t-tests or Chi-square tested differences in F\&V intake and reports of purchasing F\&V at baseline and at 12 weeks. Participants were predominantly Latino $(48.7 \%)$, females $(69 \%)$ and lowincome $(65 \%$ reported annual income less than $\$ 20,000)$. At baseline, 39\% 


\section{NUTRITION-CLINICAL}

and $32 \%$ of participants reported ever purchasing F\&V from a farmer's market in the intervention and control arm respectively. Mean baseline fruit consumption was 0.5 and 0.9 servings/day with no significant differences between arms. At 12 weeks, $81 \%$ of participants in the intervention arm compared to $48 \%$ of participants in the control arm reported purchasing from a farmer's market $(p=0.003)$. There was a minimal increase in fresh fruit intake of 0.2 servings/day in the intervention arm compared to a decrease of 0.3 servings/day in the control group $(p=0.04)$ at 12 weeks, with no change in vegetable intake. Focused education combined with a small economic incentive resulted in an increase in purchasing behavior and in fresh fruit intake. A more intense behavioral educational program combined with increased access may result in a significant impact on obesity and diabetes, particularly among low-income and racially diverse communities.

Supported by: Albert Einstein College of Medicine

756-P

Effect of a Carbohydrate-Free Diet vs. Fasting on Plasma Glucose in Type 2 Diabetes

FRANK 0. NUTTALL, RAMI M. ALMOKAYYAD, MARY C. GANNON, Minneapolis, MN, Ames, IA

It is well known that hyperglycemia improves when patients with type 2 diabetes are placed on a weight loss diet. Typically this occurs soon after the diet is implemented. Generally it is attributed to the weight loss per se. However, this rapid improvement in hyperglycemia could be the result of the lower fuel supply (calories) in the diet, or from the lower carbohydrate content in the diet. In order to differentiate these effects, we examined, in 7 subjects with untreated type 2 diabetes, the metabolic effects of a 3-day fast (which represents the severest form of calorie restriction) with the response to a calorie-sufficient, carbohydrate-deficient (<3\% carbohydrate) diet for the same duration. A randomized crossover design with a 4-week washout period was used. Both interventions were compared to the effects of the initial standard diet ( $55 \%$ carbohydrate, $15 \%$ protein, $30 \%$ fat). At the beginning of the 3rd day, the overnight fasting plasma glucose decreased from $196 \pm 16 \mathrm{mg} / \mathrm{dl}$ while on the standard diet to $160 \pm 15 \mathrm{mg} / \mathrm{dl}$ while on the carbohydrate-free diet and to $127 \pm 10 \mathrm{mg} / \mathrm{dl}$ when the subjects were not eating. The decreases were $18 \%$ and $35 \%$, respectively. The 24 -hour total integrated plasma glucose area decreased on the 3rd day from $5248 \pm 601$ $\mathrm{mg} . \mathrm{hr} / \mathrm{dl}$ on the standard diet to $3408 \pm 291 \mathrm{mg} . \mathrm{hr} / \mathrm{dl}$ on the carbohydratefree diet (35\% decrease), and to $2661 \pm 221 \mathrm{mg}$.hr/dl after 72 -hours of fasting (49\% decrease). In summary, both a carbohydrate-free diet and short-term starvation (fasting) dramatically decrease the blood glucose in people with type 2 diabetes. Of the decrease in integrated glucose area observed with fasting, $70 \%$ could be attributed to removal of carbohydrate from the diet. Our data suggest an early decrease in plasma glucose observed in patients who are placed on weight loss diet is due largely to the reduction in food energy and/or carbohydrate content (glucose) of the diet, rather than the weight loss per se.

Supported by: U.S. Dept. of Veterans Affairs

757-P

Long-Term Weight Loss Possible in Overweight DM2 Patients Even After Initiation of Therapy With Insulin Glargine

PATRICIA MOREIRA-CALI, SALVADOR A. GEZAN, Gainesville, FL

Insulin is associated with weight gain, particularly at initiation of therapy. However many overweight DM2 adults require this form of treatment. This retrospective study analyzes the weight control of 406 overweight DM2 patients who received individualized medical nutrition therapy (MNT) for 18 months. It was hypothesized that if patients received MNT while on insulin therapy, weight gain could be avoided. The 3 study groups were IN, with 49 insulin naïve patients initiating therapy with Glargine; INN with 108 patients already on Glargine and control group L with 249 patients controlling diabetes with lifestyle changes. The cohort included $57 \%$ female and $78 \%$ Caucasian, with mean age of $63 \mathrm{yrs}, 71 \%$ being obese and $29 \%$ overweight. Participants received MNT every 3 months by an RD CDE, including reduction of $500 \mathrm{kcal} /$ day and moderate carbohydrate restriction. Repeated measures model with an autoregressive error structure was used for statistical analysis and the covariate initial weight was included. For differences among groups an f-test and t-test were used. The results indicated no significant difference in weight at baseline or 18 months among the three groups. No significant differences were found in weight loss between the two insulin groups. The weight loss over 18 months was $5.4 \mathrm{~kg}$ or $5.5 \%$ decrease from initial and $6.5 \mathrm{~kg}$ or $6.6 \%$ decrease for the IN and INN groups, respectively. In contrast the lifestyle group was significantly different from both insulin groups for most time points except for months 0 and 18 and resulted in greater weight lost (8.0 $\mathrm{kg}$ or $8.8 \%$ decrease after 18 months). Diabetes control was also improved with A1c changes from baseline to 18 months: $8.7-7 \%$ for IN, 9.5-6.5\% for INN, and $6.9-6.2 \%$ for $L$ groups. In conclusion, weight loss appears possible for patients on insulin Glargine who receive MNT. Additionally, contrary to expectations, patients new on insulin therapy receiving MNT, not only can curb weight gain, but may actually attain significant weight loss.

Supported by: Sanofi

Beneficial Effects of Kimchi on Metabolic Parameters in Sub With Metabolic Syndrome

MIN SUK LEE, SO-YEON AN, JA YOUNG JEON, SEIRHAN KIM, TAE HO KIM, JA YOUNG YOON, CHANG-OK OK, HYE-KYOUNG LEE, WON-SUN HWANG, SUN JUNG CHOE, SEUNG JIN HAN, HAE JIN KIM, DAE JUNG KIM, KWAN-WOO LEE, Suwon, Republic of Korea, Goyang, Republic of Korea

Kimchi is a traditional Korean food and served as a side dish. Its fermented form is found to reduce body weight and improve metabolic parameters in overweight and obese patients. However, research about the effects of Kimchi in subjects with metabolic syndrome (MetS) is lacking. The aim of present study was to investigate if there are beneficial effects of Kimchi and additional effects of fermented Kimchi on metabolic parameters in subjects with MetS.

A total of 16 subjects with MetS were randomly assigned to two 8-week diet phases separated by a 4-week washout period. The subjects consumed either fresh or fermented Kimchi during each diet phase (crossover design). Fresh and fermented Kimchi were only different in the degree of maturity. One-day-old and 10-day-old Kimchi were defined as "fresh" and "fermented" Kimchi, respectively. Diet composition, anthropometric data, blood samplings including glucose and lipid levels, and inflammatory cytokines levels were analyzed.

After consumption of fresh or fermented Kimchi, data of body weight waist circumference, the percentage of fat, systolic blood pressure, HbA1c, HOMA-IR, and fasting insulin showed improvement. Diastolic blood pressure decreased significantly in the fermented Kimchi group (from $79.4 \pm 6.8$ to $75.7 \pm 8.8 \mathrm{mmHg}$ ) compared to the fresh Kimchi group ( $\mathrm{p}=0.037)$. C-reactive protein, the marker of inflammation, also decreased in the fermented Kimchi group (from $959.0 \pm 1013.7$ to $750.5 \pm 828.4 \mathrm{ug} / \mathrm{mL}$ ) compared to the fresh Kimchi group ( $\mathrm{p}=0.048$ )

Consumption of fresh or fermented Kimchi had beneficial effects on anthropometric parameters and glucose metabolism related parameters. There were additional effects on diastolic blood pressure and C-reactive protein with consumption of fermented Kimchi and these effects were not found with consumption of fresh Kimchi.

759-P

Modification of Whole Grain, Coffee and Red Meat Intake Affects Insulin Sensitivity and Ectopic Fat Content in Type 2 Diabetes LEJLA ZAHIRAGIC, BETTINA NOWOTNY, ANDREAS F. PFEIFFER, HANS-GEORG JOOST, PETER P. NAWROTH, STEPHAN BORNSTEIN, HANS-ULRICH HAERING, PETER NOWOTNY, ANDREAS FRITSCHE, JOCHEN SEISSLER, ANN K. FLEITMANN, JAN GROENER, MICHAEL RODEN, Düsseldorf, Germany, Nuthetal, Germany, Heidelberg, Germany, Dresden, Germany, Tübingen, Germany, Munich, Germany

Increased consumption of whole grain fibers, coffee and reduction of red meat intake are main protectors to develop T2D according to the German Diabetes Risk Score (GDRS). Whether an intervention focusing on these items is beneficial in overt T2D is unknown. We hypothesized that a diet based on the main GDRS factors increases insulin sensitivity and reduces subcutaneous (SCF), visceral (VF) and hepatocellular (HCL) fat content. We designed a prospective, randomized, controlled multicenter trial to examine the effects.

Diet- or metformin-treated patients with $\mathrm{T} 2 \mathrm{D}$ and diabetes duration $\leq 5 \mathrm{yrs}$ $\left(\mathrm{n}=14 ; 50 \pm 2 \mathrm{yrs}\right.$, body mass index $\left.36 \pm 1 \mathrm{~kg} / \mathrm{m}^{2}\right)$ were randomized to a GDRS based (GDR) or a control diet (CON).

The GDR (30-50 g/day fibers, $\geq 5$ cups coffee/day, no red meat) and the CON ( $\leq 10 \mathrm{~g} /$ day fibers, no coffee, $\geq 150 \mathrm{~g} /$ day red meat) diets were given for 8 weeks with identical macronutrient composition $(50 \%$ carbohydrates, $30 \%$ fat, $20 \%$ proteins) and caloric restriction by $300 \mathrm{kcal} /$ day. Compliance was monitored weekly including measurement of nutrient metabolites. Insulin sensitivity was assessed with hyperinsulinemic-euglycemic clamps combined with $\left[{ }^{2} \mathrm{H}_{2}\right]$ glucose. Magnetic resonance imaging and spectroscopy were used to measure SCF, VFM and HCL.

Glycemic control (A1c) improved $(p<0.02)$ in both groups (GDR: $-9 \pm 3 \mathrm{mmol} /$ $\mathrm{mol}$, CON: $-6 \pm 1 \mathrm{mmol} / \mathrm{mol})$. Both diets decreased $(\mathrm{p}<0.02)$ body weight 


\section{NUTRITION-CLINICAL}

$(-8 \pm 1 \%$ vs. $-3 \pm 0.5 \%)$, but weight loss was more pronounced in GDR $(p=0.0001$ vs. CON). Whole body insulin sensitivity slightly increased in GDR $(+1.3 \pm 0.5$ $\left.\mathrm{mg} \cdot \mathrm{kg}^{-1} \cdot \mathrm{min}^{-1}, \mathrm{p}=0.05\right)$, but not in CON $\left(+1.0 \pm 0.5 \mathrm{mg}^{\mathrm{kg}}{ }^{-1} \cdot \mathrm{min}^{-1}, \mathrm{p}=0.11\right)$.

Both GDR and CON diets decreased HCL $(-71 \pm 3 \%$ vs. $-35 \pm 10 \%, p \leq 0.05$ vs. baseline) and total (SCF+VF) body fat content (each $p=0.01$ vs. baseline). In conclusion, subtle nutritional modification differently affects insulin sensitivity underlining the roles of dietary red meat, fibers and coffee in patients with overt T2D.

760-P

Seven-Day Caloric and Saturated Fat Restriction Does Not Correct Increased Myocardial Dietary Fatty Acid Partitioning in Impaired Glucose-Tolerant Subjects

CHRISTOPHE NOLL, SÉBASTIEN M. LABBÉ, MARGARET KUNACH, SERGE PHOENIX, ERIC E. TURCOTTE, BRIGITTE GUÉRIN, ANDRÉ C. CARPENTIER, Sherbrooke, OC, Canada, Quebec, OC, Canada

We recently found increased myocardial partitioning of dietary fatty acids associated with subclinical ventricular dysfunction in impaired glucose-tolerant subjects ( $\mathrm{G}+$ ). These metabolic and functional myocardial abnormalities were significantly improved following an individually tailored one-year lifestyle intervention leading to weight loss. The aim of the present study was to determine the effect of a 7-day dietary caloric $(-500 \mathrm{kcal} / \mathrm{d})$ and saturated fat $(<7 \%$ of caloric intake) restriction (LowCal) vs. a 7-day isocaloric diet (Control), in random order with at least a 10-day washout period in between, on myocardial dietary fatty acid partitioning in IG+ subjects determined using our positron emission tomography coupled to computed tomography (PET/CT) method with the fatty acid analog 18-Fluoro-6-thiaheptadecanoic acid (18FTHA) during a standard liquid meal. 6 IG+ subjects (2M/4F) have completed the study thus far. Postprandial 18FTHA activity in circulation was not significantly different between the two interventions. Myocardial 18FTHA mean standard uptake value (SUV) at 6 hour was not significantly increased in LowCal vs. Control $(2.1 \pm 0.2$ vs. $2.7 \pm 0.2, P=0.06)$. Organ-specific partitioning of dietary fatty acids was also not significantly changed in the liver, adipose tissues or skeletal muscles in the LowCal vs. Control diet. Our preliminary results suggest that dietary changes do not explain by itself the normalization of organ-specific dietary fatty acid partitioning associated with a lifestyle-based weight loss intervention in IG+ subjects.

Supported by: CIHR (MOP53094)

761-P

The Effect of Dietary Lipids on Circulating Adiponectin: A Systematic Review With Meta-Analysis of Randomized Clinical Trials ANIZE DELFINO VON FRANKENBERG, FLÁVIA M. SILVA, JUSSARA C. DE ALMEIDA, VANESSA PICCOLI, MÔNICA SOST, FILIPE V. DO NASCIMENTO, CRISTIANE B. LEITÃO, LUCIANA L.R. REMONTI, DANIEL UMPIERRE, ANDRÉ REIS, LUIS CANANI, MIRELA J. DE AZEVEDO, FERNANDO GERCHMAN, Porto Alegre, Brazil, São Paulo, Brazil

Different dietary strategies, including lipids intake, may influence circulating adiponectin concentrations. In order to better understand this issue we identify, critically examine, summarize, and analyze studies that investigated the effects of dietary lipids (type and amount) on circulating concentrations of adiponectin. It was performed a systematic review with meta-analysis of randomized clinical trials with predefined inclusion and exclusion criteria; search of the literature in Medline, Embase, and Scopus electronic databases. The outcome was changes in fasting adiponectin concentration from baseline to the end-of-study expressed as weight mean differences. From 3,206 studies retrieved, 40 fulfilled the inclusion criteria and were grouped according to the following interventions: total dietary lipids amount (12 reports), dietary or supplementary n-3 PUFA (12 reports), conjugated linoleic acid (CLA) supplementation (7 reports), and other interventions in dietary lipids amount or quality (9 reports). Low-fat diet in comparison to control diet was not associated with changes in adiponectin concentrations (6 reports; $\mathrm{WMD}=0.30$ [95.0\% $\mathrm{Cl} ;-0.64$ to 1.25$]$ ). An increase of adiponectin with n-3 PUFA supplementation occurred with at least 2.0 $\mathrm{g} / \mathrm{d}$ supplementation (3 reports; $\mathrm{WMD}=2.37$ [95.0\% Cl; 0.43 to 4.30$]$ ). In contrast, CLA supplementation reduced circulating adiponectin (8 reports; WMD $=-0.74[95.0 \% \mathrm{Cl} ;-1.38$ to -0.10$]$ ] in comparison with unsaturated fat. Studies demonstrate that intake of at least $2.0 \mathrm{~g} / \mathrm{d}$ of $\mathrm{n}$-3 PUFA supplement increases adiponectin concentrations. However, low-fat diet and CLA supplementation does not have this beneficial effect.

Supported by: FIPE; FAPERGS; CAPES; CNPq
762-P

24h Urinary Sodium Excretion Is Associated With Serum Aldosterone in People With Diabetes

RENATA LIBIANTO, GEORGE JERUMS, QUE LAM, ANGELA CHEN, SARA BAQAR, FELICITY PYRLIS, RICHARD J. MACISAAC, JOHN MORAN, ELIF I. EKINCI, Me/bourne, Australia, Heidelberg, Australia, Adelaide, Australia

Recent studies in type 2 diabetes patients reported increased mortality associated with low $24 \mathrm{~h}$ urinary sodium excretion (24hUNa) (Ekinci et al, Diabetes Care 2011). This cross sectional study aimed to determine the relationship between $24 \mathrm{hUNa}$, plasma renin activity (PRA), serum aldosterone, and NT-proBNP in patients with diabetes. Clinical characteristics and biochemistry were recorded in 222 consecutive patients (77\% with type 2 diabetes) attending diabetes clinics at Austin Health. The relationship among $24 \mathrm{hUNa}$, serum aldosterone, PRA, NT-proBNP, urinary potassium excretion, serum potassium, serum sodium, eGFR, urinary albumin excretion, and $\mathrm{HbA1c}$ was examined by multivariable regression model. 24hUNa significantly predicted serum aldosterone in a linear fashion $(R 2=0.20, p=0.002$, Figure). In the subgroup of patients $(n=46)$ not taking renin angiotensin aldosterone (RAAS) modifying agents, this relationship was more pronounced than in the whole cohort (univariate analysis $r=-0.39$, $p=0.008$, versus $r=-0.13, p=0.04$ ), and remained significant in this subgroup in the multivariate analysis $(\mathrm{R} 2=0.10, \mathrm{p}=0.03)$. There was no demonstrable relationship between $24 \mathrm{hUNa}$ with PRA or NT-proBNP. In conclusion, low $24 \mathrm{hUNa}$ is associated with increased serum aldosterone in people with diabetes, in the presence and absence of RAAS modifying agents. This raises the possibility that stimulation of the RAAS may contribute to adverse outcomes observed with a low sodium diet.

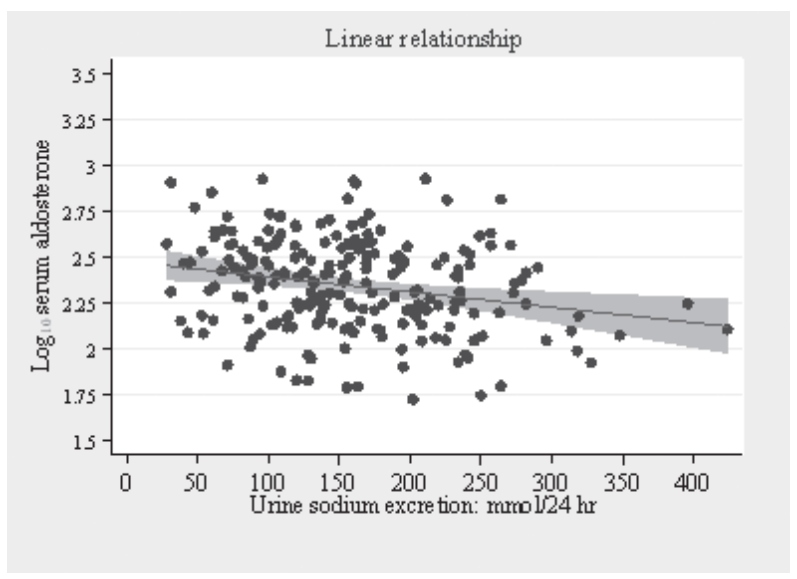

763-P

A Non-Calorie-Restricted Non-Ketogenic Low-Carbohydrate Diet Is Effective as an Alternative Therapy for Patients With Type 2 Diabetes

SATORU YAMADA, YOSHIFUMI YAMADA, JUNICHIRO IRIE, Minato, Japan

Aims: Although caloric restriction is a widely used intervention, many patients are unable to comply with such dietary therapy for a long period. The clinical effectiveness of low-carbohydrate diets was recently described in a position statement of Diabetes UK and a scientific review by the American Diabetes Association. Therefore, the aim of this study was to examine the effects of a non-calorie-restricted, non-ketogenic, low-carbohydrate diet (low-carbohydrate diet) in Japanese patients.

Materials and Methods: The enrolled patients were randomly allocated to a conventional calorie-restricted diet or a low-carbohydrate diet. Patients received consultations every 2 months from a registered dietician for 6 months. We compared the effects of the two dietary interventions on glycaemic control and metabolic profile.

Results: Haemoglobin A1c decreased significantly from baseline to 6 months in the low-carbohydrate diet group but not in the calorie-restricted group. Patients in the former group also experienced reductions in body weight and improvements in lipid profiles and diastolic blood pressure, without major adverse effects. Patients in both group reported improvements in treatment satisfaction.

Conclusions: Our findings suggest that a low-carbohydrate diet is appropriate for patients with type 2 diabetes who are unable to adhere to a calorie-restricted diet. 


\section{NUTRITION-CLINICAL}

\begin{tabular}{lccccccc}
\hline Valuables & $\begin{array}{l}\text { low-carbo- } \\
\text { hydrate } \\
\text { group }\end{array}$ & \multicolumn{5}{c}{$\begin{array}{l}\text { caloric- } \\
\text { restriction } \\
\text { group }\end{array}$} \\
\hline & before & after & $\begin{array}{l}\text { intra-group } \\
\text { comparison }\end{array}$ & before & after & intra-group inter-group \\
comparison comparison
\end{tabular}

764-P

A Randomized, Two-Regimen, Crossover, Comparative Study to Evaluate the Blood Glucose Regulation and Safety of a Resistant Starch Formula versus Commercially Available Food in Type 2 Diabetic Patients

CHIA-HUNG LIN, DAW-MING CHANG, LEE-MING CHUANG, Taoyuan, Taiwan, Pingtung, Taiwan, Taipei, Taiwan

Objective: Resistant starch has been proposed to improve postprandial glucose and diabetic control. The effect of a new resistant starch formula, PPB-R-203 (Pharma Power Biotec, Taiwan) based food is not clear.

Methods: A randomized, two-regimen, crossover, comparative study was conducted in a total of 44 type 2 diabetic patients. The participants received PPB-R-203 based or commercially available food in a random sequence and crossover with at least 2-day washout period between the two regimens. Each subject was admitted to the hospital one day before and took the study product at 07:00, 12:00 and 18:00 for another two days. Continuous glucose monitoring system (Medtronic Minimed, USA) was applied to record blood glucose levels in a non-real-time manner at each treatment period.

Results: The mean blood glucose levels decreased significantly from $141.6 \pm$ 29.8 at control to $133.6 \pm 29.1 \mathrm{mg} / \mathrm{dl}$ at PPB-R-203 regimen $(P=0.012)$. The area under curve (AUC) of total blood glucose and hyperglycemic state (sugar $>180 \mathrm{mg} / \mathrm{dl}$ ) were also remarkably reduced (from $97819.2 \pm 20691.1$ to $83791.6 \pm 20919.8, P<0.001$ and $32678.9 \pm 33006.0$ to $25180.0 \pm 29479.2$, $P=0.011$ respectively). But the AUC of hypoglycemia (blood sugar $<70 \mathrm{mg} /$ dl) was not significantly different between two regimens. The low blood glucose index (LBGI) and high blood glucose index (HBGI) for hypo- and hyperglycemia risk assessment demonstrated no difference in these two treatments. The glucose variation parameters, including mean amplitude of glucose excursion (MAGE) and M-value were both improved but not statistically significant.

Conclusions: The PPB-R-203 based food was effective for postprandial hyperglycemic control in type 2 diabetic patients. The risk of hypoglycemia was the same as the commercially available food and glucose variation was not deteriorated.

765-P

Low-Rice Intake in Low Energy Density Diet Stimulates Sweetness Desire in Women more than in Men

BEI ZHOU, HISAMI YAMANAKA-OKUMURA, CHISAKI ADACHI, YUKA KAWAKA-

MI, TAKAFUMI KATAYAMA, EIJI TAKEDA, Tokushima, Japan, Hyogo, Japan

The effective energy density (ED) diet model for customized meal plan needs to be explored further. However, food preferences between genders with ED are unknown. This study was conducted to examine the effects of lunches with different dietary EDs on sensory properties between genders.

In a randomized crossover study, 280 healthy Japanese subjects included 182 men and 98 women consumed packed meals during 6 sessions. Using the control meal as a reference test meal which included cooked rice 150 $\mathrm{g}$, sauteed beef $40 \mathrm{~g}$, vegetable $240 \mathrm{~g}, 500 \mathrm{kcal}$, ED $0.75 \mathrm{kcal} / \mathrm{g}$; high-meat/ low-rice meal, $513 \mathrm{kcal}, 0.73 \mathrm{kcal} / \mathrm{g}$; Low-vegetable meal, $427 \mathrm{kcal}, 0.99$ $\mathrm{kcal} / \mathrm{g}$; medium-fat $/ \mathrm{low}$-vegetable meal, $520 \mathrm{kcal}, 1.20 \mathrm{kcal} / \mathrm{g}$; high-fat meal, $896 \mathrm{kcal}, 1.28 \mathrm{kcal} / \mathrm{g}$; and high-fat/low-vegetable meal, $824 \mathrm{kcal}, 1.77 \mathrm{kcal} / \mathrm{g}$ were served as modified test meals with varying macronutrient distribution and ED. Subjective levels of sensory properties were assessed using visual analog scales (VAS) at 0, 0.5, 1, 2, 3, 4 and $5 \mathrm{~h}$ after meal intake.

Results showed that meals with higher vegetables provided higher satiety and satiation regardless of gender $(p<0.05)$. Although responses to meals changed within a smaller range for men than for women, men had stronger desires of salty and fatty taste than women after meals $(p<0.05)$. Women had significantly higher satiety than men until $3 \mathrm{~h}$ after meals with higher vegetables $(p<0.05)$, and also had a stronger desire of sweetness than men from $2 \mathrm{~h}$ after meals with lower vegetables $(\mathrm{p}<0.05)$. Moreover, in lowED diet of control meal with $150 \mathrm{~g}$ rice, sweetness desire was stronger in women than in men from $4 \mathrm{~h}$ post-meal $(p<0.01)$, whereas in high-meat/ low-rice meal with $100 \mathrm{~g}$ rice, sweetness desire was stronger in women than in men from $3 \mathrm{~h}$ post-meal $(\mathrm{p}<0.05)$

We concluded that increased vegetable intake is more effective to enhance satiety and satiation regardless of gender, and the desire of sweetness especially for Japanese women is also stimulated by decreasing rice intake in low-ED diet model.

Supported by: University of Tokushima

766-P

Relationships between Plasma Free Amino Acid Concentrations With Energy Intake and Insulin and Glucagon-Like Peptide-1 in Response to Increasing Loads of Intraduodenal Whey Protein in Healthy Men

AMY T. RYAN, CHRISTINE FEINLE-BISSET, STIJN SOENEN, ROBERT E. STEINERT, PETER M. CLIFTON, MICHAEL HOROWITZ, NATALIE D. LUSCOMBE-MARSH, Adelaide, Australia

There is some evidence that plasma total and branched chain amino acids (AAs) are associated with protein-induced suppression of energy intake and the release of insulin and glucagon-like peptide-1 (GLP-1), but the impact of other AAs remains unclear. This study aimed to characterize the effects of increasing intraduodenal (ID) loads of protein on the plasma concentrations of 20 free AAs, and to determine which AAs potently modulate the energy intake and insulintropic responses. In a double-blind, cross-over design, 16 healthy males [mean \pm SEM age: $27 \pm 3$ y; BMI (in $\left.\mathrm{kg} / \mathrm{m}^{2}\right)$ : $22.1 \pm 0.6$ ] had plasma free AAs and GLP-1, and serum insulin, concentrations, measured during 60-min ID infusions of hydrolysed whey at either: 1) $0.5 \mathrm{kcal} / \mathrm{min}$ (P0.5), 2) $1.5 \mathrm{kcal} / \mathrm{min}(\mathrm{P} 1.5)$, or 3$) 3 \mathrm{kcal} / \mathrm{min}(\mathrm{P} 3)$, or 4) $0 \mathrm{kcal} / \mathrm{min}(\mathrm{C})$. Energy intake was quantified at $\mathrm{t}=60 \mathrm{~min}$. Compared with C, 19/20 AAs were increased as the load of protein increased ( $\mathrm{P}<0.05$ for all comparisons), with exception of cysteine. Glutamine and asparagine, which are not present in whey, also increased in a load-dependent manner $[\mathrm{P}<0.001$ for all]. After pooling all data, within subject associations were found between the $A U C_{0-60 \text { min }}$ responses for all AAs, except cysteine, with: 1) load of protein $\left(R^{2}\right.$ varied between $0.1-0.9, P<0.05), 2)$ energy intake $\left.\left(R^{2}, 0.09-0.3, P<0.05\right), 3\right)$ insulin $\left(R^{2}, 0.3-0.6, P<0.001\right)$, and (4) GLP-1 $\left(R^{2}, 0.2-0.6, P<0.05\right)$. In conclusion, plasma AAs were related in varying degrees to the load of protein, and tyrosine, methionine, leucine, isoleucine, lysine and valine, were the strongest predictors of energy intake and the insulintropic effects. Our observations support the notion that the plasma AA pool is determined by metabolic requirements of the various peripheral organs and not simply the AA composition of a nutritive load. These findings have implications for the dietary management of a variety of metabolic disorders.

Supported by: ANHMRC (Australia) (62711820 to N.DL.M.)

767-P

The Equal Effectiveness of Carbohydrate Counting to Calorie Restriction as Dietary Management in Patients With Non-InsulinTreated Type 2 Diabetes

SHINYA FUKUMOTO, MASANORI EMOTO, MASAYUKI HOSOI, SHIGEICHI SHOJI, TOMOYUKI KAWAMURA, KOKA MOTOYAMA, TOMOAKI MORIOKA, KATSUHITO MORI, TETSUO SHOJI, MASAAKI INABA, 4C STUDY INVESTIGATORS, Osaka, Japan

Basic carbohydrate (carb) counting can be an easy-to-use and effective diet therapy for patients with type 2 diabetes (T2D). However, unlike for strict low-carb diets, it is unknown whether mild control of carb can lead to proper restriction of calories, lipids and proteins. We evaluated the efficacy of carb counting as a dietary therapy in patients with $\mathrm{T} 2 \mathrm{D}$ in this multicenter, randomized, non-blind, parallel-group comparative study registered in advance (UMIN000001588). The study included patients with non-insulintreated T2D without renal dysfunction. Two hundred and one patients were enrolled in the study, and were divided into a carb counting group and calorie counting group. The calorie group was instructed to restrict calorie intake to $25-30 \mathrm{kcal} / \mathrm{kg}$ ideal body weight (IBW)/day, and the carb group was instructed to ingest carb amounts corresponding to dietary energy amounts of $50 \%$ to $60 \%$ at $25-30 \mathrm{kcal} / \mathrm{kg} \mathrm{IBW/day.} \mathrm{Dietary} \mathrm{instructions} \mathrm{were} \mathrm{given}$ by registered dietitians at the start of the study, after 1 month, and after 3 months. The primary endpoint was A1C level, and the secondary endpoints were other metabolic and lipid profiles, levels of adipokines, nutrient intake, and treatment satisfaction (DTSQ). A1C levels 6 months after the start of the study showed no significant difference between the calorie and carb groups. 


\section{NUTRITION-CLINICAL}

BMI values of both groups significantly reduced 6 months after the start of the study, but the values showed no significant difference between the 2 groups. In addition, lipid profiles showed no significant difference between the groups. On the other hand, the findings from an examination of patients completing the dietary instruction protocol showed that the carb group had a significant A1C decrease, while the calorie group had no significant reduction in $\mathrm{A} 1 \mathrm{C}$ levels. In conclusion, the carb counting is equally effective as the calorie restriction for patients with non-insulin-treated T2D.

Supported by: Grant-in-Aid for Scientific Research

768-P

Protein-Rich Meal Replacement Significantly Reduces HbA1c, Weight and Antidiabetic Medication in Type 2 Diabetes Patients: A Randomized Controlled Trial

STEPHAN MARTIN, BABETTE GÄRTNER, RUDOLF KEIL, KERSTIN KEMPF, Düsseldorf, Germany, Grevenbroich, Germany

Formerly, we demonstrated in an uncontrolled proof-of principle study that protein-rich meal replacement (PRMR) was successful in reducing daily insulin demand, $\mathrm{HbA1}$ c and weight in type 2 diabetes mellitus (T2DM) patients injecting $>100 \mathrm{U}$ insulin/day. The aim of the present study was to investigate in a randomized-controlled trial if PRMR is also effective in T2DM patients treated with oral antidiabetic medication or insulin.

77 T2DM patients had been randomized into two groups. During the 1st week the intervention group with stringent diet regime $(n=40)$ replaced 3 main meals by $50 \mathrm{~g}$ PRMR (Almased-Vitalkost, Almased Wellness GmbH, Bienenbüttel, Germany) each (=1100 kcal/day). In 2nd-4th week 2 meals were replaced and a protein-rich lunch was allowed. In 5th-12th week only dinner was replaced. The control group with moderate diet regime $(n=37)$ replaced breakfast and dinner for 5 weeks and then only dinner during the next 7 weeks. Clinical parameters were determined at the study center at baseline, after 4,8 and 12 weeks. Primary endpoint was reduction of $\mathrm{HbA1c}$, secondary endpoints reduction of weight and antidiabetic medication. Differences were determined by Mann Whitney and Wilcoxon signed rank test.

Baseline values of the participants (60\% men, age $60 \pm 12$ years) did not differ significantly. $82.5 \%$ of the stringent and $81.0 \%$ of the moderate group completed the 12-week program. Both groups significantly reduced their $\mathrm{HbA1}$; ; in the stringent group $-0.8 \pm 1.4 \%$ from $8.6 \pm 1.4 \%$ to $7.9 \pm 1.6 \%$ $(p<0.001)$ and in the moderate group $-0.5 \pm 1.2 \%$ from $8.5 \pm 1.4 \%$ to $7.9 \pm 1.2 \%$ $(p=0.02)$. Both groups significantly reduced weight $(-8.4 \pm 6.9 \mathrm{~kg} ; p<0.001 \mathrm{vs}$. $-5.5 \pm 5.9 \mathrm{~kg} ; p<0.001$ ) and antidiabetic medication (both $p<0.001$ ).

In sum, both diet regimes with PRMR had been effective in reducing $\mathrm{HbA1c}$, weight and antidiabetic medication in T2DM patients, although with the stringent diet regime a stronger reduction had been achieved with comparable dropout rate.

Supported by: Almased Wellness GmbH

769-P

Investigation of Resting Energy Expenditure (REE) Using a Metabolic Analyzer in Diabetic Nephropathy (DN)

YUKO KAWAKAMI, TERUKO SATO, MAMIKO HITOMI, TATUMI MORIYA, Sagamihara, Japan

Dietary protein, salt, and energy intake in medical nutrition therapy for DN in Japan are set according to renal function. Energy intake is recommended higher than diabetic patients without DN, sometimes over $30 \mathrm{kcal} / \mathrm{kglBW}$ when renal function is decreased. However, there is no scientific evidence of energy setup in particular since objective data were not obtained previously and the energy settings recently begin to be reexamined. Therefore, we measured the amount of REE using the metabolic analyzer in 34 diabetic outpatients with various degree of renal function ( 26 men, age $60 \pm 10$ y.0,HbA1c $7.6 \pm 1.4 \%$, BUN/Cr 32.4 $22.1 / 2.18 \pm 1.76 \mathrm{mg} / \mathrm{dL}$, and estimated GFR $40.0 \pm 28.8 \mathrm{~mL} / \mathrm{min} / 1.73 \mathrm{~m}^{2}$ ) to clarify essential energy intake in DN. Actual dietary intake was estimated from $24 \mathrm{hr}$ collected-urine and dietitians' interview using food records. Body weight (BW), a rate of body fat, and lean body mass (LBM) were also examined. REE, measured before lunch was $1501 \pm 358 \mathrm{kcal} / \mathrm{day}$ and $25.5 \pm 5.3 \mathrm{kcal} / \mathrm{kglBW}$. Basal energy expenditure (BEE) calculated using the Harris Benedict equation was $1440 \pm 190 \mathrm{kcal} /$ day, and REE/BEE was $104 \pm 19 \%$. BW and body mass index (BMI) were $71.0 \pm 10$.3 $\mathrm{kg}$ and $26.7 \pm 4.0 \mathrm{~kg} / \mathrm{m}^{2}$, respectively and they did not change for 3 months before and after the REE measurements. Rate of body fat was $27.4 \pm 10.3 \%$, the muscle volume was $47.2 \pm 10.1 \mathrm{~kg}$, and LBM was $50.9 \pm 8.6 \mathrm{~kg}$. REE significantly correlated with LBM $(r=0.61, p<0.01)$. Daily energy intake using food records in 20 patients was $1450 \pm 218 \mathrm{kcal}$ that was $109 \pm 25 \%$ of REE $(1387 \pm 307 \mathrm{kcal})$, $104 \pm 15 \%$ of $B E E$ and $25.5 \pm 48.7 \mathrm{kcal} / \mathrm{kglBW}$. BW was maintained with energy intake similar to their REE during 6 months observation. The estimated daily protein intake using $24 \mathrm{hr}$ collected-urine was $0.94 \mathrm{~g} / \mathrm{kg} \mathrm{IBW}$. In conclusion, REE measurement might be useful for setting dietary energy, and REE of DN patients was about $104 \%$ for BEE. Therefore, energy setting as $25 \mathrm{kcal} / \mathrm{kg}$ IBW for DN patients may be reasonable.

770-P

Improving Metabolic Outcomes-The Academy of Nutrition and Dietetics (A.N.D.) Evidence-Based Guidelines for Those at Highest Risk for Type 2 Diabetes (T2DM)

PATRICIA DAVIDSON, SHELLEY MESZNIK, KARI KREN, Westfield, NJ, Mount Kisco, NY, Chicago, IL

The epidemic of diabetes places the spotlight on those at highest risk for T2DM and the need for nutrition guidelines. The objective of this systematic review was to develop Prevention of Type 2 Diabetes Evidence-Based Nutrition Practice Guidelines using the A.N.D.'s evidence analysis process.

Those at the highest risk for T2DM were identified using established definitions (prediabetes per ADA, metabolic syndrome per ATP III and WHO). Questions included a problem, intervention, comparison and clinical outcome. Inclusion criteria consisted of RCT or Cohort design, analysis that controlled for weight loss in non-weight loss questions and populations without T2DM. Conclusion statements and recommendations were formulated and graded based on the strength of the supporting evidence (Grade I-V and StrongInsufficient Evidence).

Impact on metabolic outcomes was analyzed for the following nutrition factors: medical nutrition therapy (MNT) (6 studies), carbohydrate entities and glycemic index/load (8 studies), macronutrient distribution (19 studies), weight loss (59 studies).

MNT led to significant decreases in fasting blood glucose, A1C, triglycerides, weight, waist circumference and systolic blood pressure and an increase in HDL cholesterol with metabolic syndrome, and a significant decrease in postprandial and fasting glycemic outcomes with prediabetes. Low glycemic index/load meals decreased blood pressure significantly in those with metabolic syndrome and glycemic-related outcomes in those with prediabetes. Blood pressure and anthropometric outcomes were improved by weight loss in those with metabolic syndrome.

MNT, including interventions to promote weight loss and appropriate diet composition, is crucial in treating those at highest risk for T2DM. Registered dietitians, within the care team, can provide MNT suited for this population utilizing the evidence-based nutrition practice guidelines.

Supported by: Academy of Nutrition and Dietetics

771-P

Effects of Long Term Intermittent Fasting on Biochemical and Homeostasis Parameters (Ramadan Research Group Prospective Study)

INES KHOCHTALI, ILHAM HELLARA, TAHER CHAKROUN, OLFA HARZALLAH, ZOHRA DRIDI, SONIA HAMDI, MOHSEN HASSINE, SEMIR NOUIRA, Monastir, Tunisia, Sousse, Tunisia

Effect of long term intermittent fasting (LIF) on metabolic and homeostasis is poorly studied.

The aim of this study is to evaluate the effect of long term intermittent fasting ( 2 days a week during 6 months) on biochemical and homeostasis parameters.

Patients and methods: Forty six healthy volunteers were included and randomly assigned in two groups: Group LIF $(n=23)$ who performed a fasting on two fixed days per week during 6 months (Monday and Thursday) and Group no LIF (N = 23).

Biochemical (glycaemia, uricemia, total cholesterolemia, ApoB, ApoA, high-density lipoprotein cholesterol (HDL-c) , Low-density lipoprotein (LDL c), triglyceridemia, C-reactive protein (CRP)) And homeostasis (Prothrombin Time, Factor VII, PFA-100, Kaolin Cephalin Time) parameters were performed at baseline (before fasting) and 6 months later.

Paired tests comparison was performed between pre and post fasting values in both groups.

Results: glycaemia decreased more in LIF group more than the no LIF ( $p<$ 0.01 ). Total cholesterolemia, Apo B, LDL-c and CRP were reduced significantly after fasting in LIF group $(p<0.05)$.

The other biochemical parameters were not significantly changed from baseline in both groups.

PFA and platelets did not significantly changed from baseline, while Factor VII decreased significantly in LIF group only $(p=0.02)$.

In conclusion: Long term intermittent fasting seems to be beneficial with regard to its effect on glycaemia, ApoB, LDL-c (metabolic effect), uricemia (oxydatif stress), CRP (inflammatory marker) and factor VII ( thrombotic risk). 


\section{NUTRITION-CLINICAL}

$\Delta$

Patterns of Sweet Taste Likings

772-P

KEIKO ASAO, JASON MILLER, LEANN ARCORI, JULIE LUMENG, THERESA HANMARKEY, WILLIAM HERMAN, Ann Arbor, MI, East Lansing, MI

Taste preferences are associated with food choices and may impact the development of obesity, which is a major risk factor for type 2 diabetes mellitus. Two distinct patterns of sweet taste preferences have been described: one showing a monotonic increase of liking at progressively higher sucrose concentrations and the other showing a peak liking in the mid-range of sucrose concentrations. However, classification of the patterns has been somewhat arbitrary. In this report, we analyzed patterns of sweet taste likings in 26 healthy men and women, age 18 or older, using cluster analysis. In the analysis, we included 14 women and 12 men, $32.6 \pm 14.5$ years of age with body mass index $26.4 \pm 5.1 \mathrm{~kg} / \mathrm{m}^{2}$ (mean \pm S.D.). Sweet taste liking was measured for 10 levels of sucrose solutions ( $0.035 \mathrm{M}$ to $1.346 \mathrm{M}$ ). Participants rated their liking for each solution using a computerized visual analogue scale with 0 as the lowest liking and 100 as the highest. The cluster analysis demonstrated two distinct groups: 13 who preferred high sucrose concentrations and 13 who preferred relatively low sucrose concentrations and showed lesser liking for high concentrations. If we use the $0.598 \mathrm{M}$ sucrose solution only and a cutoff liking score of 50 , we can distinguish the two clusters with high sensitivity $(100 \%)$ and specificity $(100 \%)$. If validated in additional studies, this simple tool may help us to better understand eating behaviors with clinical and public health interventions for obesity, and dietary modification for diabetic patients.

773-P

A Brown Rice Diet Improves Vascular Function in Patients With Type 2 Diabetes Mellitus

KEIKO KONDO, KATSUTARO MORINO, ATSUSHI ISHIKADO, KEIKO NAKAO, SAKIYA YOSHIMOTO, OSAMU SEKINE, TAKESHI YOSHIZAKI, SATOSHI UGI, ATSUNORI KASHIWAGI, HIROSHI MAEGAWA, Otsu, Japan

The intake of dietary fiber is inversely related to the cardiovascular event. It has been reported that the vascular function is decreased at postprandial state. We hypothesized that the improvement of vascular function may be a mechanism of cardioprotective effect with the high fiber diet. Therefore, we compared the effect of brown rice and white rice on vascular function in patients with type 2 diabetes mellitus.

Eleven diabetic patients (65.1 years, $M / F=5 / 6$, diet $/ 0 H A=9 / 2$ ) were randomly assigned to 2 groups of either consume brown rice $(n=6)$ or white rice $(n=5)$ for 8 weeks. The meal tolerance test and the vascular function with reactive hyperemia using strain-gauge plethysmography at the fasting and postprandial state were performed before and after the intervention. In the brown rice group, the intake of dietary fiber during the intervention period was higher than that in the baseline $(12.7 \pm 4.4$ vs. $20.4 \pm 3.7 \mathrm{~g}, \mathrm{p}<0.0001)$. The vascular function assessed with flow debt repayment (FDR) and peak forearm blood flow (FBF) tended to be decreased at the postprandial state both in the white rice group and the brown rice group before intervention $(-22 \%, p=0.11$ vs. $-24 \%, p=0.01 ;-20 \%, p=0.16$ vs. $-13 \%, p=0.41)$. Further decrease were observed in the white rice group but not in the brown rice group after the intervention in $\operatorname{FDR}(-35 \%, p=0.016$ vs. $-17 \%, p=0.24)$ and in peak FBF $(-37 \%, p=0.009$ vs. $-5 \%, p=0.86)$. Overall, a brown rice diet improves the vascular function compare to the white rice.

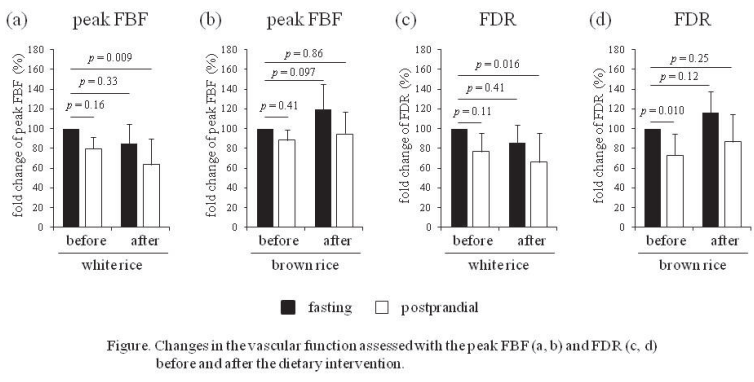

774-P

Regional Adiposity and Insulin Resistance: Insights from the Ketogenic Diet in Children

SIMONA BERTOLI, ALBERTO BATTEZZATI, ILARIA GIULINI NERI, CLAUDIA TREN-

TANI, ANNA TAGLIABUE, Milan, Italy

Ketogenic Diet (KD) is an isocaloric high-fat (80-90\%), low carbohydrate (2-5\%) diet applied effectively for treatment of refractory childhood epilepsy, pyruvate dehydrogenase complex deficiency and GLUT1 deficiency. KD induces ketone bodies production to support brain energy metabolism. We considered KD an unique model to evaluate in human the "overflow hypothesis" proposed by Bergman et al (Obesity. 2006;14:16S-19S) about the development of visceral adiposity, hyperinsulinemia, and insulin resistance after isocaloric high fat diet in the dog model. Body composition by anthropometry, subcutaneous (SAT) and visceral abdominal fat (VAT) by ultrasonography, glucose and lipid metabolism were evaluated before and after 12 weeks of $K D$ in 7 children (mean age: $8,4 \pm 2,0$ ). The table reports the time course of the main variables investigated

In 12 weeks KD did not change BMI z-scores, VAT, SAT and total body fat whereas it significantly reduced fasting glucose, insulin and HOMA and increased QUICKI. No significant changes occurred on lipid metabolism. Thus, increasing fat in the diet without achieving an hypercaloric intake did not increase visceral and subcutaneous abdominal fat and did not cause peripheral insulin resistance in the short term in children. Longitudinal studies are needed to provide a conclusive answer on the adaptive metabolic changes on regional adiposity and insulin resistance occurring in humans during isocaloric high fat diet.

\begin{tabular}{|c|c|c|c|c|c|c|c|}
\hline & z-score BMI & SAT & VAT & Gly & Ins & Homa & QUICKI \\
\hline & & $\mathrm{mm}$ & $\mathrm{mm}$ & $\mathrm{md} / \mathrm{dl}$ & $\mathrm{uU} / \mathrm{ml}$ & & \\
\hline Baseline & $0,19 \pm 1,4$ & $1,21 \pm 1,7$ & $2,9 \pm 1,1$ & $84,3 \pm 8,8$ & $5,5 \pm 2,8$ & $1,1 \pm 0,6$ & $0,38 \pm 0,03$ \\
\hline 12 weeks & $0,16 \pm 1,2$ & $1,10 \pm 1,7$ & $3,3 \pm 0,7$ & $75,7 \pm 6,1^{*}$ & $2,6 \pm 1,6^{*}$ & $0,5 \pm 0,3^{*}$ & $0,38 \pm 0,03^{*}$ \\
\hline
\end{tabular}

BF: body fat; Gly:glycemia; Trig:triglycerides; HOMA; fasting glucose mg/ $\mathrm{dL}^{*}$ fasting insulin $\mu \mathrm{U} / \mathrm{mL}$; QUICKI: 1 / (loglfasting insulin $\mu \mathrm{U} / \mathrm{mL}$ ) + log(fasting glucose $\mathrm{mg} / \mathrm{dL})$ ). ${ }^{*} p<0,05$. all values are means $\pm \mathrm{sd}$

775-P

A Plant-Based Diet Reduces Depression and Anxiety and Improves Work Productivity: The GEICO Multicenter Trial

ULKA AGARWAL, SURUCHI MISHRA, NEAL D. BARNARD, JIA XU, SUSAN LEVIN, JOSEPH GONZALES, CAROLINE TRAPP, Washington, DC

Health in the workplace is often thought of in physical aspects, such as the presence or absence of injuries or illnesses than can diminish work productivity. However, people in the workforce are often encumbered by problems in psychological adjustment, most notably depression, but anxiety as well. We sought to determine whether a low-fat plant-based nutrition program in a multicenter, corporate setting improves indices of depression, anxiety, and work productivity. Two hundred and ninety-two employees from ten sites of a major US company with a body mass index (BMI) $\geq 25 \mathrm{~kg} / \mathrm{m} 2$ and/or a previous diagnosis of type 2 diabetes were asked to either follow a low-fat vegan diet and receive weekly group instruction, or make no diet changes and receive no instruction for 18 weeks. In both an intention-totreat and an analysis for study completers, the vegan group had significantly more improvement in measures of depression, anxiety, fatigue, emotiona well-being, and work impairment compared with the control group at 18 weeks. We conclude that an 18-week dietary intervention using a low-fat vegan diet in a multicenter, corporate setting can reduce depression and anxiety, and increase work productivity of employees.

776-P

Influence of Short-Term High-Protein Diet and Low Glycemic Index Diet on Body Mass and Composition in Overweight and Obese Subjects

EWA WALILKO, MALGORZATA U. NAPIERALA, MARTA E. BRYSKIEWICZ, LILIANNA MAJKOWSKA, Police, Poland

Due to the rising problem of obesity, various weight reduction diets are widely spread and applied. The aim of the study was to compare the effectiveness of high-protein diet and low glycemic index diet.

The study group consisted of 35 overweight and obese healthy subjects (BMI $33,6 \pm 4.2 \mathrm{~kg} / \mathrm{m}^{2}$ ), aged $41,4 \pm 11,01$ years, who throughout a period of $8 \pm 2$ weeks followed a reduction diet restricted by $700 \mathrm{kcal} /$ day as compared to their usual diet evaluated based on dietary records. The participants were randomly assigned to group $\mathrm{G1}$, in which the initial diet was high-protein diet

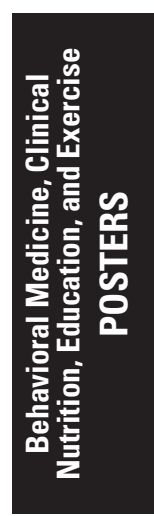


(HP), followed by low glycemic index $\operatorname{diet}(\mathrm{LGI})$, or to group $\mathrm{G} 2$, where the diet was first LGI, and then HP (each for the duration of $4 \pm 1$ weeks). All subjects did not change their usual level of physical activity for the duration of the study. During 3 control visits (week 0, 4 and 8 ) body mass, BMI, hip and waist circumference, and body composition were measured (TANITA BC-418-MA analyzer).

After 8 weeks, in both groups, a reduction of body mass, BMI, waist and hip circumference $(p<0,001)$, adipose tissue $(p 1<0,0001, p 2<0,0002)$ and nonadipose tissue $(p 1<0,001, p 2<0,002)$ were observed, as well as an increase of body water content, significantly higher in group $\mathrm{G} 1(\mathrm{p}<0,04)$. A significantly higher final reduction of body mass $(-6,0 \pm 2,4 \mathrm{~kg} \mathrm{v} .-4,8 \pm 3,2 \mathrm{~kg}, \mathrm{p}<0,05)$, BMl $\left(-2,1 \pm 0,8 \mathrm{~kg} / \mathrm{m}^{2}\right.$ v. $\left.-1,7 \pm 0,9 \mathrm{~kg} / \mathrm{m}^{2}, p<0,05\right)$, and adipose tissue $(-4,7 \pm 2,1 \mathrm{~kg}$ v. $-3,0 \pm 3,2 \mathrm{~kg}, \mathrm{p}<0,03$ ) was observed in group $\mathrm{G} 1$. In both groups, the first diet applied gave better results than the one applied afterward. Applying the LGI diet after the HP diet gave better results than HP diet after LGI.

High protein diet and low glycemic index diet are similarly effective, if they are the first diet applied in order to reduce body mass; however, applying the high protein diet at the beginning of therapy gives better final results after a change for another diet.

777-P

Efficacy and Tolerance of a Diabetes Specific Formula in Patients With Type 2 Diabetes Mellitus: An Open Label, Randomized, Crossover Study

SEEMA GULATI, ANOOP MISRA, RAVINDRA M. PANDEY, KRITI NANDA, VIVEK GARG, SANJEEV GANGULY, LORENA CHEUNG, Delhi, India, Gurgaon, India, Kowloon, Hong Kong

Type 2 diabetes mellitus (T2DM) is a huge burden and a public health concern in Asian populations. Medical nutrition therapy is the cornerstone in the management of T2DM, but, scientific evidence is lacking on the effect of Diabetes specific formulas, in this population. An open label, randomized, crossover study was conducted to evaluate the role of a diabetes specific formula, in the maintenance of blood glucose, insulin levels in the Indian diabetic population

In the study, 40 patients with T2DM were enrolled. After screening, eligible patients were counseled on diet and exercise regime during runin period. After run-in period, patients were randomized to receive either a diabetes specific formula, Nutren ${ }^{\circledR}$ Diabetes, Nestlé Nutrition, Switzerland (Group A) or isocaloric diet (Cornflakes and Milk, Group B) after overnight fasting. Blood samples were collected for estimation of Blood Glucose, Insulin and Triglyceride levels at stipulated time points $(0,30,60,120$, and $180 \mathrm{~min}$ ) after consumption. The subjective sensations of hunger, satiety, and other appetite sensations were also assessed using a Visual Analog Scale (VAS) Questionnaire to evaluate tolerance. The study was registered at www.ctri.nic.in with Reg. No. CTRI/2011/10/002089.

The postprandial glucose response i.e. Area under curve at 30, 60, 120 , \& 180 min was significantly lower with Group A as compared to Group B $(p=0.0003,0.0001,0.0001,0.0001$, respectively). Increase in serum insulin levels from baseline was also lower for Group A at 120 and 180 min respectively as compared to Group $B(p=0.0001,0.0002$ respectively). There was no significant difference in mean triglyceride levels and Visual Analog Scale questionnaire score between the two groups.

These results suggest that the Diabetes specific formula (Nutren ${ }^{\circledR}$ Diabetes) can play a significant role in nutritional management of T2DM with better maintenance of post-meal blood glucose levels compared with an isocaloric diet.

\section{PSYCHOSOCIAL, BEHAVIORAL MEDICINE}

Guided Audio Tour: Health Behaviors in Persons With and at Risk For Diabetes (Posters: 778-P to 785-P), see page 19.

T2D Genetic Risk Counseling \& Testing in Primary Care ALEX CHO, ALLISON VORDERSTRASSE, SUNIL SUCHINDRAN, JOSEPH LUCAS, WILLIAM MICHAEL SCOTT, MARYLOU BEMBE, DANA BAKER, SUSANNE B. HAGA, LORI ORLANDO, GLORIA M. TRUJILLO, SCOTT V. JOY, GEOFFREY S. GINSBURG, Durham, NC

The clinical utility of implementing T2D genetic risk testing combined with traditional risk factors (e.g., family history, ethnicity/race, prediabetes, overweight/obesity, diet, exercise) in primary care is unknown and behavioral effects (i.e., diet, exercise) of T2D genomic risk information have not been well studied. We implemented risk counseling in 2 primary care clinics; consenting patients were randomized to: 1) genetic results and standard T2D risk assessment (SRA+G), or 2) SRA alone, both more intensive than standard of care. DNA testing included 4 single nucleotide polymorphisms (SNPs) associated with T2D (rs1801282, rs10811661, rs7756992, rs7903146). Here we report clinical and behavioral study outcomes. Baseline measures included weight, HOMA-IR, family history, health behaviors, attitudes, and demographics. At 3 and 12 months post risk counseling, clinical (weight) and behavioral (diet, activity) measures were repeated. Linear mixed models were used to test for differences in weight, diet and physical activity at 3 months between groups. 409 patients enrolled. Mean age was $49.9 \pm 13.3$ years; $70 \%$ were female. $58 \%$ self-identified as White, $29 \%$ African American, and 13\% other. Rates of prediabetes, obesity, and family history of T2D were high. At 3 months, both groups significantly improved dietary habits (increased fruit/vegetable servings [0.3; $s e=0.11 ; p=0.007$ ]; decreased fatty foods $[-0.12 ; \mathrm{se}=0.06 ; p=0.05]$ ), with no difference between groups. There was no significant change in physical activity. Both groups had clinically significant weight loss (4.7 lbs- 95\% Cl 2.8-6.5; $p<0.001$ ) with no difference between groups. Participants reduced risk factors for T2D after risk counseling, regardless of receiving genetic risk information. T2D risk visits with providers have a short-term effect on patient behaviors and clinical measures; genetic risk information did not have an additive impact. We will report long-term (12 month) outcomes and the long-term impact of genetic risk counseling and testing.

Supported by: The Duke Endowment

Factors Motivating Individuals to Consider Genetic Testing for Type 2 Diabetes Risk Prediction

JENNIFER WESSEL, JYOTI GUPTA, MARY DE GROOT, Indianapolis, IN

Whole genome sequencing has created opportunities to translate genetic information to patients. To identify attitudes predicting desire for genetic testing to predict type 2 diabetes (T2D) risk, we surveyed $n=599$ adults $18-55$ years without self-reported diabetes. The majority of respondents were $37 \pm 11$ years, BMI $28+9 \mathrm{~kg} / \mathrm{m}^{2}$. White $(54 \%)$, female $(69 \%)$, with some college education (53\%), and annual income below $\$ 25,000$ (44\%). Most participants were interested in engaging in genetic testing for T2D (52\%) and $47 \%$ strongly/agreed genetic testing should be available to the public. Significant predictors of desire to engage in genetic testing were: high perceived personal risk for T2D, greater worry about risk, high perceived utility of the test, positive family history of T2D, perceived health status as fair, and knowledge that $\mathrm{T} 2 \mathrm{D}$ is preventable. In multivariate analyses, adjusting for age, gender and obesity, predictors included: positive family history $[\mathrm{OR}=1.79(1.12,2.87)]$, high perceived risk $[\mathrm{OR}=3.75(1.79,7.86)]$, knowing risk prior to disease onset $[\mathrm{OR}=4.91(3.08,7.84)]$, knowledge that T2D is preventable [OR=1.97 $(1.25,3.10)]$, possible future genetic treatment for T2D $[O R=2.20(1.31,3.70)]$ and increased motivation if the test is accurate $[\mathrm{OR}=1.70(1.05,2.77)]$. Among those not interested in testing $(n=284), 50 \%$ $(p<0.0001)$ and $63 \%(p<0.0001)$ became interested, respectively, if testing was free or they had knowledge that $\mathrm{T} 2 \mathrm{D}$ is preventable. With major advances in discovery and technology, genetic testing is becoming more routine for the early identification of T2D risk. In our sample of younger adults, individuals were generally receptive to genetic testing in the presence of perceived personal risk and worry and knowledge that diabetes could be successfully prevented. These data suggest that genetic testing coupled with education about diabetes prevention has the potential to motivate individuals to reduce their risk of T2D

Supported by: Indiana University

Weight Loss Practices Among Overweight/Obese People With Pre-
diabetes, NHANES, 2005-2010
YANFENG LI, LINDA GEISS, EDWARD GREGG, Atlanta, GA
Weight loss is the most effective strategy to prevent or delay type 2
diabetes, but the levels of participation in various weight control strategies
among U.S. adults with prediabetes is not known. We estimated the
percentage of persons with prediabetes who tried to lose weight in the
past year and described their adopted weight loss strategies. We examined
2603 prediabetic (100=<FPG<126mg/dl or $5.7 \mathrm{mg} / \mathrm{ml}=<A 1 C<6.4 \mathrm{mg} / \mathrm{dl})$ adults
aged 20 years participating in $2005-2010$ National Health and Nutrition
Examination Survey. Prediabetes awareness was measured by self-report
among people who met lab test criteria. We examined whether prediabetic
persons reported trying to lose weight in the last year and grouped their
reported weight loss strategies into 3 non-mutually exclusive groups




\section{PSYCHOSOCIAL, BEHAVIORAL MEDICINE}

dietary change (including ate less food, etc.), exercise, and other (including took diet pill, etc.). Data were analyzed using SAS and SUDAAN. About $36 \%$ of people had prediabetes. Overall, $43.2 \%$ of overweight or obese adults with prediabetes reported trying to lose weight in the last year. Among those trying to lose weight, $91.2 \%$ used strategies involving dietary change, $55.5 \%$ involving exercise, $62.5 \%$ using other, and half using both dietary change and exercise. Weight loss behavior was positively associated with prediabetes awareness [odds ratio 1.75(95\% Cl: 1.21-2.53)] after adjusting for age, sex, race, education and body mass index. Non-Hispanic blacks, men, and persons with low education were less likely to report trying to lose weight. Younger people were more likely to report trying to lose weight than people aged 75 or older. Fewer than half of overweight/obese persons with prediabetes reported trying to lose weight and, among those who tried, only half used both diet and exercise strategies. Prediabetes awareness was significantly associated with action to lose weight. These findings suggested a need to improve awareness of prediabetes status and effective approaches to prevent diabetes in high risk adults.

ก 781-P Age and Social Media Awareness as Predictors for Technology Utilization in Diabetes Care

MARIA I. CONSTANTINO, TIM MIDDLETON, MARGARET MCGILL, JENCIA WONG, DENNIS K. YUE, TED WU, Sydney, Australia

Information technology (IT) has the potential to improve the quality, safety and efficacy of diabetes management, however its success in improving outcomes is determined by patient acceptance of IT. To evaluate these attitudes in the Australian diabetes community, a 20 question selfadministered survey instrument was distributed to people attending selected diabetes ambulatory clinics in multiple urban and rural areas.

Of 312 respondents, $57.1 \%$ were males, $65.9 \%$ resided in Sydney, $22.8 \%$ in rural areas (11.3\% undisclosed), $68.3 \%$ were aged $\geq 50$ years old and $63.8 \%$ had type 2 diabetes. Significant positive attitudes were observed in the comfort levels of using IT in general and comfort in integrating IT into diabetes care, but there were differences in types of IT accepted and across all age groups. While the use of email in diabetes care was accepted by $71 \%$ of subjects, web/video based consultations were acceptable by only $45 \%$ of subjects. There was a strong downwards trend of comfort with either of these technologies as age increased $(p<0.0003)$.

Social media awareness (SMA) was gauged by ability to recognize the Facebook or Twitter logos. SMA was significantly associated with the use of downloading data from blood glucose monitors $(p=0.004)$. When analysed by logistic regression, age $<50$, having type 1 diabetes and living in a rural/ regional setting were the major independent predictors for using meter downloads ( $p<0.02$ ). Only $50 \%$ of subjects are using internet health searches and $73.3 \%$ did not want/undecided about joining an online diabetes support group. However, the SMA positive subjects were more than twice as likely to want to join such a group $(p<0.0001)$.

Patient attitude toward integration of IT into diabetes care is generally favorable, particularly in the younger age groups. SMA predicts increased likelihood for the usage of other forms of IT in their management. These predictors could be used to better target which patients are more likely to successfully integrate IT into their diabetes care.

\section{ก 782-P}

Validation of an Information-Motivation-Behavioral Skills (IMB) Model of Diabetes Medication Adherence

LINDSAY S. MAYBERRY, CHANDRA Y. OSBORN, Nashville, TN

Suboptimal medication adherence is common and is associated with poor outcomes, but few theories of diabetes medication adherence are available to inform interventions. The IMB model of adherence has been validated to predict adherence to antiretrovirals, hypertension medications, and treatments for heart patients, so we examined its predictive utility in diabetes.

We recruited adults with type 2 diabetes $(\mathrm{N}=314)$ who were prescribed oral agents/insulin to complete measures of adherence-related information (DMKQ), motivation (MDQ), behavioral skills (MASES-R), and behavior (ARMS-D and SDSCA medications subscale). A nurse collected point-ofcare A1C. A structural equation model estimated relationships between IMB model constructs.

Participants were age $51.8 \pm 11.7$ years; $65 \%$ female; $53 \%$ Black; $32 \%$ had $<$ high school degree; and $45 \%$ had incomes $<\$ 10 \mathrm{~K}$. Average A1C was $8.2 \% \pm 2.2 \%$. The estimated IMB model had excellent data fit, and the IMB variables explained $39 \%$ of the variance in adherence. Information and motivation were related to adherence through behavioral skills (both indirect effects, $p \leq$.05), information and behavioral skills were directly related to adherence (both $p<.05)$, and adherence was related to A1C ( $p<.001$; see figure).

Adherence promotion interventions should aim to enhance patients' adherence-related information, motivation (attitudes), and behavioral skills since doing so would improve both adherence and glycemic control.

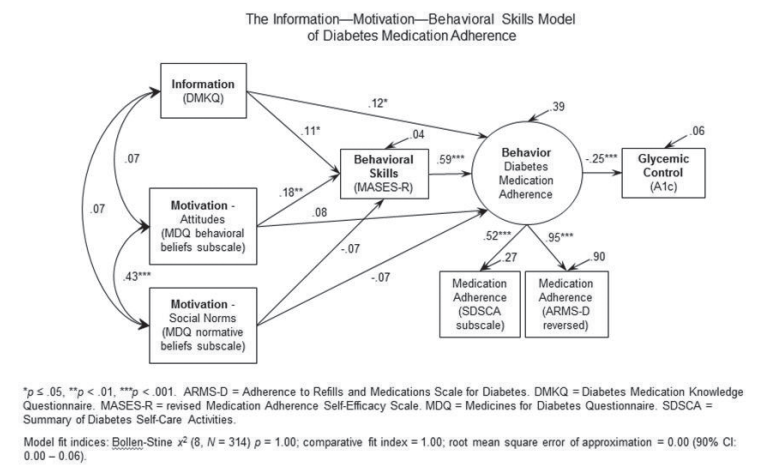

Supported by: NIH (UL1TR000445): NIDDK (K01DK087894 to C.Y.O.), (F32DK097880 to L.S.M.)

ก 783-P

Regimen Related Distress, Medication Adherence, and Glycemic Control in Rural African American Women With Type 2 Diabetes Mellitus

DOYLE M. CUMMINGS, LESLEY LUTES, KERRY LITTLEWOOD, EMILY DINATALE, BERTHA HAMBIDGE, KATHLEEN SCHULMAN, LATOYA VINES, Greenville, NC

Emotional distress in patients with Type 2 diabetes is associated with poor glycemic control but the mediators of this relationship, especially in rural African American women who experience disproportionate levels of psychosocial stress, are not well described. The purpose of this study was to examine the cross-sectional relationship between diabetes-specific regimen-related distress, medication adherence, and $\mathrm{HbA1c}$, in $\mathrm{n}=202$ rural African American women (mean age $=53 \pm 11$ yrs, 47\% high school education or less, $79 \%<\$ 30,000 / \mathrm{yr}$., $\mathrm{BMI}=37.7 \pm 8.2$ ) in the southeastern US enrolled at baseline in the EMPOWER randomized trial. Regimen related distress was measured as a subscale of the validated 17-item Diabetes Distress Scale. Medication adherence was measured using the validated 8-item Morisky Medication Adherence Scale, and $\mathrm{HbA1C}$ was measured via fingerstick sample using a DCA Vantage Analyzer. Regimen related distress scores, medication adherence scores, and $\mathrm{HbA1c}$ were all highly correlated $(p \leq 0.01)$. Fifty-six percent of women reported elevated levels of regimen-related distress and these women had significantly lower mean medication adherence scores (4.5 vs. 6.4, $p<0.001$ ) and significantly higher mean $\mathrm{HbA1c}$ values (9.6 vs. 8.6, $\mathrm{p}<0.001$ ). In multivariate modeling that controlled for both the duration of diabetes and the number of visits in the last year, both regimen-related distress $(\beta=0.22,95 \% \mathrm{Cl} 0.056-0.50, p<$ $0.05)$ and medication adherence $(\beta=-0.20,95 \% \mathrm{Cl}-0.33--0.02, \mathrm{p}<0.05)$ were independently associated with poor glycemic control ( $\mathrm{HbA1c})$. Among rural African American women with Type 2 diabetes, elevated levels of regimen-related distress was highly prevalent and was associated with both inadequate medication adherence and poor glycemic control. Regimenrelated distress may be associated with poor glycemic control in part via interfering with medication adherence.

Supported by: Bristol-Myers Squibb

ก 784-P

The Association between Depressive Symptoms and Diabetes SelfCare in Low Income African American and Latino Patients With Type 2 Diabetes in a Primary Care Setting

ROSALBA HERNANDEZ, LAURIE RUGGIERO, THOMAS PROHASKA, NOEL CHAVEZ, NADINE PEACOCK, ARIE NOUWEN, Chicago, IL, Fairfax, VA, Birmingham, United Kingdom

There is a paucity of published studies exploring the association between depressive symptoms and diabetes self-care (DSC) practices among minority adults with low socioeconomic status residing in the U.S. This study aimed to examine the relationship between depressive symptoms and DSC in African American and Latino adults with type 2 diabetes mellitus (T2DM). Baseline in-person survey data was collected from African Americans and Latinos aged $\geq 18$ years with T2DM participating in a diabetes self- 
management intervention at four primary care clinics. Depressive symptoms were assessed using the Patient Health Questionnaire and the Summary of Diabetes Self-care Activities was used to assess performance levels of healthy eating, physical activity, glucose monitoring, foot care, and smoking. The sample $(n=276)$ had a mean age of 53.2 years; $69 \%$ were women; $54 \%$ African American and 46\% Hispanic; and 74\% reported incomes below $\$ 20,000$. Using multivariate regression techniques, each of the self-care subcomponents was regressed on the independent variable of depression while controlling for age, ethnicity/race, income, and BMI. For the total sample of African American and Latino patients with T2DM, depressive symptoms were negatively associated with the DSC behaviors of general diet $(\beta=-0.026, p=0.0001)$, specific diet $(\beta=-0.024, p=0.0003)$, physical activity ( $\beta=-0.027, p=0.001)$, foot care $(\beta=-0.017, p=0.005)$ and smoking $(\beta=-$ $0.060, p=0.04$ ), with higher depression scores associated with lower selfcare performance. These findings underscore the importance of assessing depressive symptomatology in people with diabetes and including a focus on emotional well-being when working to improve diabetes outcomes.

Supported by: National Institute of Nursing Research (5R01NR010313)

\section{$\Delta$}

ก 785-P

Skype-Based Family Problem Solving for Youth With Poorly Controlled Diabetes: Relative Effectiveness of Improving Adherence and Metabolic Control

MICHAEL A. HARRIS, KURT A. FREEMAN, DANNY C. DUKE, BETH HIRSCHFIELD, BRUCE BOSTON, Portland, OR

This study examined the relative effectiveness of family-based problem solving for adolescents with poorly controlled diabetes. Participants received Behavioral Family Systems Therapy for Diabetes (BFST-D) delivered via video-conferencing (SKYPE) or face-to-face (CLINIC) to youth with poorly controlled type 1 diabetes $(\mathrm{HbA} 1 \mathrm{c}<9.0 \%)$ and their families.

At Baseline, Post-treatment, and Follow-Up (3-months), adolescents and their parent(s) completed the Diabetes Self Management Profile (DSMP), an assessment of diabetes treatment adherence. Glycemic control (HbA1c) was evaluated at three time points, Pre, Post and Follow-up. Overall mean $\mathrm{HbA1c}$ at enrollment was $11.01 \%(S D=1.67 \%)$ and duration of diabetes was 6.7 years $(S D=3.57)$. Mean participant age was 15.0 years $(S D=1.77), 40 \%$ were female, and $89 \%$ were Caucasian. Dropout rates for BFST-D were 3 of 46 (SKYPE) and 2 of 44 (CLINIC). At least 1 treatment session and all 3 evaluations were completed by 28 youth and families randomized to SKYPE, while 33 youth and families randomized to CLINIC completed at least 1 treatment session and 3 evaluations.

Two separate ANCOVAs were conducted with BFST-D serving as the independent variable with baseline $\mathrm{HbA1}$ c values and youth-reported DSMP total scores as covariates. An intent-to-treat design included using the last available data to impute missing data points. From baseline to the 3-month follow-up, HbA1c for the CLINIC group declined by an average of .59\%, while that for the SKYPE group declined by an average of .34\%. No significant differences between groups were identified. From baseline to the 3-month follow-up, DSMP scores for the CLINIC group increased by an average of 5.91 points, while those for the SKYPE group increased by an average of 2.97 points. ANCOVA yielded no significant between group differences. These findings suggest that BFST delivered by videoconferencing is equal in effectiveness to traditional BFST delivered in person.

Guided Audio Tour: Psychosocial Issues among Youth and Young Adults with Diabetes (Posters: 786-P to 793-P), see page 19.

\section{6-P}

Attention Deficit Hyperactivity Disorder (ADHD) Impacts Diabetes Management and Glycemic Control among Adolescents and Young Adults With Type 1 Diabetes (T1D)

KELLEE M. MILLER, ROY W. BECK, LORI M. LAFFEL, JOSEPH LARGAY, SATYA SHANMUGHAM, HENRY RODRIGUEZ, KATHERINE MANSEAU, GEORGEANNA J. KLINGENSMITH, JILL H. SIMMONS, WILLIAM V. TAMBORLANE, Tampa, FL, Boston, MA, Chapel Hill, NC, Stanford, CA, Aurora, CO, Nashville, TN, New Haven, CT

This analysis assesses the effect of ADHD on T1D management and glycemic control among 4,540 adolescents and young adults, ages 13-<26 yrs, participating in the T1D Exchange clinic registry (median age $16.7 \mathrm{yrs}$, duration 7.0 yrs, $49 \%$ female, $82 \%$ white). Participants (pts) were classified as ADHD not on medication (meds), ADHD on meds, or no ADHD. Groups were compared in logistic and linear regression models for: self-monitoring of blood glucose (SMBG) /day, missed insulin dose $\geq 1$ time/week, HbA1c, and diabetic ketoacidosis (DKA) and severe hypoglycemic (SH) events in the past 3 months.

Overall, 460 (10\%) pts reported a diagnosis of ADHD of whom $51 \%(235)$ currently took meds. Mean SMBG/day was 4.5, 4.9, and 5.0 in the ADHD not on meds, ADHD on meds, and no ADHD groups, respectively ( $P=0.17)$. ADHD pts on meds $(41 \%, \mathrm{P}<0.001)$ or not $(38 \%, \mathrm{P}=0.003)$ were more likely to report missing insulin doses compared with no ADHD pts (30\%). Mean HbAlc was higher in ADHD not on meds $(9.0 \%, P=0.003)$ but not $A D H D$ on meds $(8.7 \%, P=0.06)$ compared with no ADHD pts (8.6\%). Frequency of $\mathrm{SH}$ was higher among ADHD not on meds ( $P=0.001)$ but not $A D H D$ on meds $(P=0.19)$ compared with no ADHD, whereas DKA frequency was higher for ADHD not on meds ( $P=0.01$ ) and on meds ( $P=0.04$ ) compared with no ADHD (Figure)

ADHD adversely affects diabetes management and clinical outcomes. T1D outcomes in patients with ADHD, with and without treatment, should be further assessed.

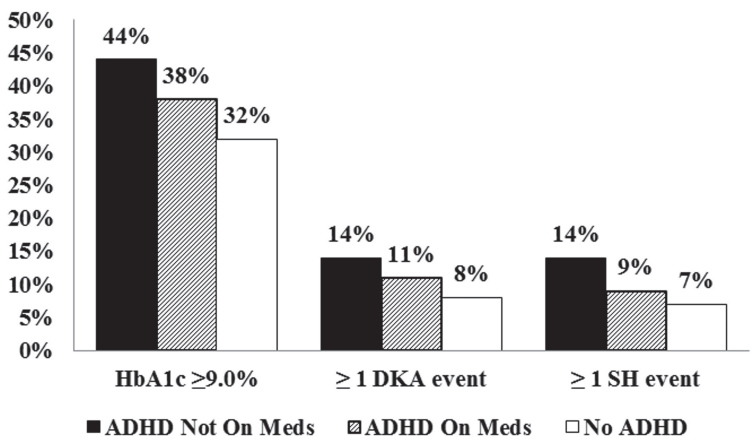

Supported by: Leona M. and Harry B. Helmsley Charitable Trust

ก 787-P

Impact of Mental Health (MH) on Fear of Hypoglycemia (FOH), Glycemic Control, and Quality of Life (0OL) in Youth With Type 1 Diabetes (T1D)

KARA R. HARRINGTON, JESSICA T. MARKOWITZ, LISA K. VOLKENING, MICHELLE L. KATZ, LORI M. LAFFEL, Boston, MA

$\mathrm{FOH}$ impacts glycemic control and $\mathrm{OOL}$ in $\mathrm{T1D}$ and relates to symptoms of anxiety and depression. To study associations among these constructs in pediatric patients, T1D youth (49\% male), ages 8-17 years ( $\mathrm{N}=93)$, completed surveys of MH constructs (FOH survey (Cox, 1987), STAI-C (anxiety), CES-DC (depression), and PedsOL (generic QOL)). Youth were $12.7 \pm 2.7$ years old with T1D duration $5.9 \pm 3.6$ years; A1c was $8.0 \pm 0.9 \%, 86 \%$ were pump treated. In bivariate analyses, depressive symptoms $(r=.43)$ and trait anxiety $(r=.54)$ were directly related to $\mathrm{FOH}$ (both $\mathrm{p}<$.0001). No $\mathrm{MH}$ constructs were related to $\mathrm{A1c}$. $\mathrm{FOH}(\mathrm{r}=-.43)$, state anxiety $(\mathrm{r}=-.43)$, trait anxiety $(\mathrm{r}=-.68)$, and depressive symptoms ( $r=-.65$ ) were inversely related to $00 \mathrm{~L}$ (all $p<.0001)$. In multivariate analyses, trait anxiety $(\beta=.68, p=.03)$ and depressive symptoms $(\beta=.49, p=.01)$ predicted $\mathrm{FOH}$. No MH constructs predicted A1c. State anxiety $(\beta=-.42$, $p=.05)$, trait anxiety $(\beta=-1.15, p=.0001)$, and depressive symptoms $(\beta=$ $-.39, p=.004$ ) predicted $00 \mathrm{~L}$. A mediation model demonstrated that trait anxiety and depressive symptoms mediated the effect of $\mathrm{FOH}$ on $\mathrm{OOL}$. Together, $\mathrm{FOH}$, anxiety, and depressive symptoms explained $73 \%$ of the variance in youth-reported $00 \mathrm{~L}$ ( $p<.0001$ ) (Figure). Symptoms of affective disorders appear to underlie $\mathrm{FOH}$ in T1D youth. As clinical efforts focus on improving A1c in T1D youth, screening for affective disorders and offering MH support may preserve youth QOL. 
Mediation Models of Quality of Life

A

\begin{tabular}{|c|c|c|}
\hline $\begin{array}{c}\text { Fear of } \\
\text { Hypoglycemia }\end{array}$ & $\begin{array}{c}\beta=-0.54 \\
p<.0001\end{array}$ Quality of Life \\
\cline { 2 - 3 } &
\end{tabular}

B

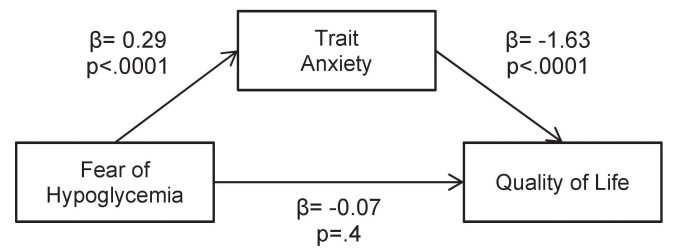

C

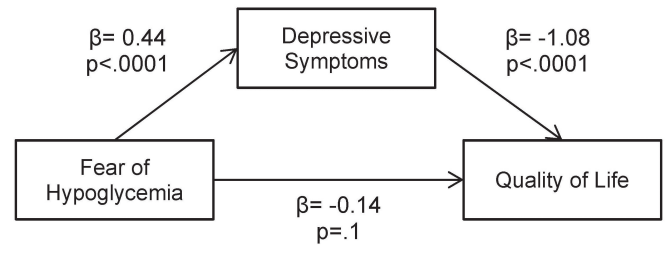

Supported by: NIH/NIDDK (1R01DK089349-01A1)

ก 788-P

Mealtime Behavior Problems Account for More Variation in Mean Daily Glucose Control than Healthy Eating in Young Children With Type 1 Diabetes

SUSANA R. PATTON, LAWRENCE M. DOLAN, ASHLEY MOORE, SCOTT W. POW-

ERS, Kansas City, KS, Cincinnati, $\mathrm{OH}$

Adoption of intensive insulin therapy in young children means that children no longer need to follow a strict schedule for mealtimes or consume a fixed amount of carbohydrates at meals. However, despite these changes, parent report of child mealtime problems remains common and children do not consume a healthy diet. To determine how to prioritize intervention strategies, we examined how child mealtime behavior problems and healthy eating compare in explaining variations in glycemic control among young children. A sample of 39 families (child age: $5.1 \pm 1.2$ years; HbA1c: $8.6 \pm 1.3 \%$ ) participated. Parents completed the Behavioral Pediatric Feeding Assessment Scale (BPFAS) to measure child behavior. To evaluate children's diet, parents completed a 3-day diet record which was scored using the Healthy Eating Index-2005 (HEl-2005). Children's glycemic control was measured using continuous glucose monitoring and was concurrent with parents' assessment of child behavior and diet. Correlations revealed significant relations between children's mean daily glucose levels and the BPFAS child problem score $(r=0.47, p=0.01)$ and the HEI-2005 $(r=-0.34$. $p=0.03$ ). Children's BPFAS child problem score and HEI-2005 were also significantly related $(r=-0.40, p=0.02)$. In a partial correlation controlling for the HEl-2005, a strong positive correlation remained between children's BPFAS child problem score and their daily glycemic control $(r=0.41, p=0.02)$. In contrast, correlating HEI-2005 and daily glycemic control, while controlling for BPFAS child problems, there was no correlation ( $r=-0.09, p=0.63$ ). While child mealtime behaviors and a healthful diet both relate to daily glycemic control, our findings suggest child behavior may be superior to healthy eating in explaining variations in children's glycemic control. Thus, interventions focused on improving glycemic control may want to focus on mealtime behavior problems over healthy eating.

Supported by: DK076921

ก 789-P

Mediators of Externalizing Behavior and Glycemic Control in Youth With Type 1 Diabetes

MEGAN L. MCCORMICK KING, RACHEL SWEENIE, ELEANOR MACKEY, RANDI STREISAND, CLARISSA HOLMES, Washington, DC, Richmond, VA

Adolescents with type 1 diabetes (T1D) are at increased risk for experiencing externalizing behavior problems (e.g., oppositional behavior, rule breaking, attention problems), and these symptoms are associated with poorer glycemic control. This study sought to explore possible mediators in the relationship between externalizing symptoms and glycemic control, in particular nutrition and adherence/self-care behaviors. Baseline data were analyzed from a randomized control trial (RCT) aimed at preventing decline of glycemic control in young adolescents. The sample consisted of 257 adolescent-parent dyads $(\mathrm{M}$ age $=12.8 \pm 1.2,49.4 \%$ female, $\mathrm{M} \mathrm{HbA1c}=$ $8.8 \pm 1.6)$ who completed measures of adolescent externalizing symptoms, adherence and self-care behaviors, and nutrition. A1c data were extracted from patients' medical charts. Structural equation modeling (SEM) analyses were conducted to examine the relations among externalizing, nutrition, adherence behaviors, and glycemic control. For externalizing symptoms, the model demonstrated good fit, $\chi 2(160)=236.78, p<.001, \mathrm{CFI}=.93, \mathrm{RMSEA}=$ $.05, \mathrm{SRMR}=.06$. The indirect path between externalizing symptoms, adherence behaviors, and A1c was significant $(b=-.20, p=.01)$, while the path between externalizing symptoms, nutrition, and A1c was non-significant $(b=-.12, p=.18)$. As seen in prior research, externalizing behaviors negatively impact glycemic control in adolescents with T1D. Further, it appears that this relationship may occur by directly impacting adherence/self-care behaviors. In contrast, nutrition may be less affected by externalizing symptoms. It is possible that such behavioral issues impede adolescents' ability to carry out self-care behaviors, as well as caregivers' ability to supervise and ensure good adherence. Clinical attention should be paid to possible underlying psychopathology, such as externalizing behavior problems, when caring for adolescents with poor glycemic control.

Supported by: NIH

ก 790-P

Examining Family Structure: The Impact of Single Parent Status and Family Density on Glycemic Control in Youth With Type 1 Diabetes LAURA J. CACCAVALE, KATHRYN E. MAHER, ELIZABETH M. ROBINSON, ADRIENNE P. BORSCHUK, ZACHARY RADCLIFF, ANIL KUMAR, RUSAN CHEN, RANDI STREISAND, CLARISSA S. HOLMES, Richmond, VA, Washington, DC

Youth with type 1 diabetes (T1D) from single parent families are in poorer glycemic control (HbA1c). However, demographic trends indicate increased households composed of unmarried adults and fewer youths. Family density or a youth: adult ratio may be a more salient factor related to glycemic control. Data were from 257 adolescents aged 11-14 yrs $(M=12.84)$ at two different sites. Single-parent status was determined by parental report of a sole caregiving adult in a youth's household. A family density ratio was calculated via parental report of the number of youths to adults in a home. A youth: adult ratio greater than two was considered "higher family density" (Liaw \& Brooks-Gun, 1994). Glycemic control was determined via a DCA2000 analyzer with results abstracted from medical chart review. The ADA (2010) recommends HbA1c levels for adolescents below 7.5\%. Single-parent status was correlated with higher $\mathrm{HbA1c}(r=.19, p=.01)$. Consistent with the literature, adolescents in single-parent families $(\mathrm{N}=53)$ were in poorer glycemic control $(\mathrm{M} \mathrm{HbA1c}=9.41 \%)$ compared to those in two-parent homes $(\mathrm{M}=8.65 \%, \mathrm{p}=.01)$. Higher family density $(\mathrm{N}=28)$ also was related to higher $\mathrm{HbA1c}(r=.32, p<.001)$. Adolescents from higher density families were in poorer glycemic control $(\mathrm{M} \mathrm{HbA1c}=10.30 \%)$ compared to those from lower density families ( $\mathrm{M}=8.61 \% \mathrm{p}=.004)$. An overall multiple regression with single-parent status, family density, SES, and ethnicity accounted for $18.8 \%$ of the variance in glycemic control. However, family density, $\beta=.28$, and SES, $\beta=-.29$, were the only significant correlates of glycemic control in the model. Although single-parent families have youths in poorer glycemic control, higher family density appears to be a more potent indicator of youth glycemic control perhaps because it may be a more sensitive indicator of available parental time and resources.

Supported by: NIDDK (5R01DK070917)

\section{ก 791-P}

Parental Monitoring and Regimen Modifications in Relation to Glycemic Variability and HbA1c in Youth With Type 1 Diabetes ADRIENNE BORSCHUK, KATHERINE MAHER, ELIZABETH ROBINSON, LAURA J. CACCAVALE, ZACHARY RADCLIFF, ANIL KUMAR, RUSAN CHEN, RANDI STREISAND, CLARISSA HOLMES, Richmond, VA, Washington, DC

More parental monitoring (PM) is related to better glycemic control $(\mathrm{HbA1c})$ in adolescents; however, mechanisms of this relation are not well understood. The current study examined relations among PM, regimen modifications, daily glycemic variability (GV) and HbA1c in a sample of 71 youth (12-16 yrs; $M=14.4$ ). PM was assessed with the Parental Monitoring of Diabetes Care scale (PMDC). Disease care modifications were assessed with the Modification subscale of the Diabetes Behavior Rating Scale (DBRS) and daily GV was evaluated with the Average Daily Risk Range from downloaded meter data. Regressions showed more parent-and youthreported PM related to better $\mathrm{HbA1c}(\beta=-.26, p=.008)$ and to more regimen 
modifications ( $\beta=.22, p=.022$ ). More modifications also related to better $\mathrm{HbA1c}(\beta=-.27, p=.006)$ which partially mediated the relation between more PM and better glycemic control (see figure). Some of the beneficial association of PM with A1c can be operationalized at least in part by more regimen modifications. More regimen modifications also related to lower daily GV $(r=-.25, p=.035)$, such that less daily glycemic variability in the high risk range is another plausible mechanism by which more PM relates to better $\mathrm{HbA} 1 \mathrm{c}$. A better understanding of factors related to the beneficial association of PM with $\mathrm{HbA1c}$ could help refine treatment programs designed to improve adolescent disease care.

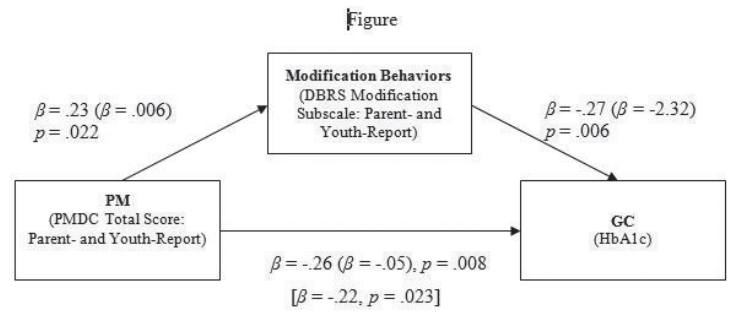

Figure 1. More modification behaviors as a partial mediator of the relation between more PM and better GC (HbAlc). Values shown are standardized regression coefficients. Values in parentheses represent unstandardized beta weights. Values in brackets account for the relation of $\mathrm{PM}$ on HbAlc after controlling for modification behaviors

Supported by: NIH

$\Delta$

ก 792-P

Comparing Family Planning Vigilance in Adult Women With Type 1 Diabetes (T1D)

SUSAN M. SEREIKA, JENNIFER THURHEIMER, ABIGAIL WILHITE, PATRICIA SCHMITT, A.B. POWELL, ANA DIAZ, DOROTHY BECKER, DENISE CHARRONPROCHOWNIK, Pittsburgh, PA

Unplanned pregnancies with diabetes could cause maternal and fetal complications. Women avoiding pregnancies should be vigilant in using effective family planning. This study compares levels of vigilance with family planning behaviors [e.g., preconception counseling (PC), using birth control (BC)] of women with T1D. This secondary analysis uses baseline data from a long-term prospective cohort study of adult women with T1D ( $N=112)$ who were re-contacted having previously participated in a PC intervention study as an adolescent (READY-Girls) and a matched comparative group of women with T1D who did not receive PC as teens. Participants completed bi-annual online questionnaires regarding self-care management, family planning behaviors and reproductive health outcomes. The current sample ( $n=88$ ) was between 18-38 years of age (mean=24), 98\% Caucasian, $82 \%$ had at least some college, $72 \%$ had a husband/boyfriend, $27 \%$ were married, $27 \%$ had $\geq 1$ biological children, $85 \%$ had private health insurance, and $49 \%$ were Roman Catholic. Only 23\% reported having received preconception counseling and care. Seventy-seven percent $(n=68)$ have been sexually active, with a mean age of sexual debut of 18.5 years (range: 15-28). Of these, $99 \%$ had used some form of $\mathrm{BC}$, but only $55 \%$ were vigilant using $\mathrm{BC}$ every time they had sex when not planning a pregnancy.

Compared to women who did not use $\mathrm{BC}$ every time, more vigilant women used condom and hormone combination BC method (44\% vs. $69 \%, p=.045$ ), had a younger mean age (years) when first received PC (23.5 vs. 18.3, $p=.04$ ), and had discussed PC with health providers (49\% vs. $73 \%, p=.09$ ); while just having received PC was not significant (47\% vs. $53 \%, p=.83$ ). Women with T1D avoiding pregnancies should be extra vigilant with $\mathrm{BC}$; however, almost half of our sample was not. Vigilant women were more likely to have had PC earlier and used more effective family planning

Further analyses are warranted to determine the most effective delivery of PC to enhance family planning vigilance in women with diabetes.

ก 793-P

Clinical Communication during Pediatric Diabetes Visits: Relations With Quality of Life, Parenting Stress and HbA1c

JENNIFER BLOSSOM, TIM WYSOCKI, JUDITH L. ROSS, HOLLY ANTAL, Philadelphia, $P A$, Jacksonville, $F L$

Much research has explored relations between glucose control and psychosocial outcomes for youth with diabetes but it is unknown how communication during clinic visits may influence these associations. This study explored relations between clinical communication and health outcomes. Forty-four audio-video recordings of pediatric diabetes visits were coded using the Roter Interaction Analysis System (RIAS) coding scheme. Parents and children completed the Peds- $\mathrm{OL}$, a quality of life measure providing Physical and Psychosocial summary scores. Parents completed the Pediatric Inventory for Parents (PIP), a measure of frequency and difficulty of parenting stress. Child's HbA1c was collected at the visit. Relations between the 11 RIAS composite scores of clinician communication and the questionnaires were assessed using Pearson correlation coefficients. Significant correlations were entered into regression equations to further elucidate the relation between variables. Clinician use of activation and facilitation (e.g. asking opinions or permission) was significantly negatively related to child-reported Peds-OL Psychosocial summary score $(B=-.37$, $\mathrm{p}=.01)$. Clinician social discussion was positively correlated with parentreported Peds-OL Psychosocial summary score $(\mathrm{B}=.31, \mathrm{p}=.05)$ and negatively related to PIP Frequency $(B=-.32, p=.03)$ and Difficulty $(B=-.30, p=.05)$ scores. Clinician use of reassurance and approval statements was significantly negatively correlated with parent-reported Peds-OL Physical Health (B $=-.37, p=.014)$ and positively related to PIP Frequency $(B=.34, p=.005)$ and Difficulty $(B=.30, p=.056)$ scores. Clinician questions regarding lifestyle or psychosocial issues were positively correlated with child's $\mathrm{HbA1c}(\mathrm{B}=.34$, $\mathrm{p}=.024)$. Clinical communication significantly correlates with psychosocial outcomes in a pediatric diabetes sample. This ongoing longitudinal study will further illuminate these relations.

Supported by: Nemours Foundation

Guided Audio Tour: Biological Processes and Psychosocial Problems in Diabetes (Posters: 794-P to 801-P), see page 19.

ก 794-P

Selective Cognitive Decline Is Related to Focal Brain Volume Loss in Type 1 Diabetes Patients With Microangiopathy: A 4-Year FollowUp

EELCO VAN DUINKERKEN, FREDERIK BARKHOF, MARTIN KLEIN, FRANK J. SNOEK, RICHARD G. IJZERMAN, MICHAELA DIAMANT, Amsterdam, Netherlands

Cross-sectional studies showed cognitive and structural brain changes in type 1 diabetes (T1DM) patients, predominantly in those with peripheral microangiopathy. Whether these brain changes progress over time is not well known

In 25 T1DM patients with microangiopathy (baseline age: $46.1 \pm 7.8 \mathrm{yrs}$ and A1C: $7.9 \pm 1.0 \% ; 40 \%$ male; I0: $112 \pm 12.7$ ) and 25 matched controls (baseline age: $44.3 \pm 8.5 y$ rs and $A 1 C: 5.4 \pm 0.3 \% ; 52 \%$ male; $10: 109.4 \pm 11.0$ ) we assessed general cognitive ability, memory, information processing speed, executive functions, attention, motor and psychomotor speed at baseline and followup. A 3D-T1 structural MRI-scan at both time points was used to determine whole-brain volume loss, analyzed with FSL-SIENA. Group differences and associations over time were analyzed with regression analyses corrected for baseline age, sex and either baseline cognitive performance, total brain volume or both.

After 4 years, patients versus controls showed significantly greater decline in executive functions (patients: $\delta z=-0.402 \pm 0.63$ sd vs. controls: $\delta z=-$ $0.007 \pm 0.39$ sd; $P=0.008$ ). Furthermore, patients versus controls had a larger percentual whole-brain volume loss (patients: $-1.34 \pm 1.0 \%$ vs. controls: $-0.68 \pm 0.63 \% ; P=0.015)$, most marked in the right frontal and central areas. In all participants, larger loss of frontal and central brain volume was related to accelerated executive functions decline $(\beta=-0.330 ; \mathrm{P}=0.025)$. In patients, higher baseline $\mathrm{A} 1 \mathrm{C}$ was associated with larger executive performance decline ( $\beta=-0.407 ; P=0.043)$ and higher baseline systolic blood pressure was correlated to frontal brain volume loss at follow-up ( $\beta=-0.501 ; P=0.011)$.

At 4-years follow-up, loss of executive performance and brain volume was significantly greater in T1DM patients with microangiopathy compared to healthy controls. Poorer glycemic control and higher systolic blood pressure at baseline predicted both cognitive and brain alterations over time.

Supported by: EFSD

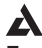

ก 795-P

Executive Function, Frustration With Self-Care and Depressive Symptoms are Associated With Glycemic Control

ELIZABETH A. BEVERLY, WOUTER S. HOOGENBOOM, SHANE M. FITZGERALD, JONATHAN S. BISHOP, MASAKAZU HIROSE, NINA F. LEWIS-SCHROEDER, KELLY M. BROOKS, OM P. GANDA, A. ENRIQUE CABALLERO, MEDHA MUNSHI, KATIE WEINGER, Boston, MA

The impact of executive function on glycemic control and self-care is unclear. We examined executive function among people with diabetes and its relationship with glycemic control and self-care. We assessed 433 adult 
patients with type 1 or type 2 diabetes $(84 \%$ White, $53 \%$ female, $55 \pm 12$ years old, $16 \pm 2$ years of education, $\mathrm{A} 1 \mathrm{C}=7.7 \pm 1.5 \%, 18 \pm 13$ years duration, $62 \%$ type 2 diabetes). We measured: executive function using Denman Scoring for the Rey-Osterrieth Complex Figure, frequency of self-care (SCI-R), diabetesrelated distress (PAID), frustration with self-care (SMO), depressive and anxiety symptoms (BSI), and quality of life (DOOL). We built two sets of multivariate linear regression models: the first model addressed frequency of self-care and the second model addressed glycemia. The first regression model found higher scores of executive function were associated with lower A1C levels (st $b=-0.09, p=0.03$ ), controlling for age, gender, type of diabetes, and years of education. More frustration with self-care (st $b=0.52$, $p<0.001$ ) and depressive symptoms ( $s t b=0.16, p=0.01$ ) were associated with higher A1C levels. This model accounted for $34 \%$ of the variation in A1C. The second regression model found higher scores of frustration with selfcare were associated with fewer self-care behaviors (st $b=-0.52, p<0.001$ ), controlling for age, gender, type of diabetes, and years of education; scores of executive function (st $b=0.017, p=0.68$ ), anxiety symptoms ( $s t b=-0.044$, $\mathrm{p}=0.47$ ) and depressive symptoms ( $\mathrm{st} b=-0.1 .05, \mathrm{p}=0.09$ ) were not associated with frequency of self-care. This model accounted for $34 \%$ of the variation in self-care. In sum, lower executive functions, frustration with self-care and depressive symptoms may be barriers to achieving glycemic targets. Diabetes patients who are struggling with glycemia, self-care and negative affect may need more highly structured programs and targeted psychosocial support to improve diabetes outcomes.

Supported by: NIDDK (2R01DK60115-06)

\begin{abstract}
$\Delta$
Enhanced Rewarding Effects of Nicotine as Assessed by Place Preference Procedures in a Rodent Model of Diabetes

JOSEPH A. PIPKIN, JESUS A. JURADO, IVAN TORRES, OSCAR V. TORRES, LUIS M. CARCOBA, ARBI NAZARIAN, LAURA E. O'DELL, EI Paso, TX, Pomona, CA

Ongoing work in our laboratory has shown that diabetic rats display an increase in nicotine self-administration and suppressed dopamine systems as compared to healthy controls. These findings have lead to the question of whether enhanced nicotine intake in diabetic rats is related to an overcompensation for suppressed dopamine systems or enhanced rewarding effects of nicotine. To address this question, we compared the rewarding effects of nicotine in diabetic and healthy control rats using place preference procedures. Separate groups of rats received a systemic injection of streptozotocin (STZ; $45 \mathrm{mg} / \mathrm{kg}$ ) or vehicle. STZ is toxic to insulin-producing cells in the pancreas and produces high glucose levels of approximately 500 $\mathrm{mg} / \mathrm{dl}$. Fourteen days after diabetes induction, all rats were tested for their initial preference for 2 distinct compartments of a conditioning apparatus. The rats were conditioned 5 days later during which time the rats received an injection of saline or nicotine ( 0.1 or $0.2 \mathrm{mg} / \mathrm{kg}$ ) and were placed into their initially non-preferred side. On alternate days, they received saline and were placed into the alternate side. After 8 days of conditioning, the rats were tested for their preference for 5 consecutive days. The results revealed that nicotine produced a shift in place preference for the compartment where the rats received nicotine as compared to rats that received saline. Diabetic rats displayed a larger shift in preference to the nicotine-paired compartment versus controls. Although preference behavior decreased over time in both groups, the greater preference behavior in diabetic rats persisted across the 5 test days. Taken together with our self-administration data, our results suggest that the rewarding effects of nicotine are enhanced in diabetic rats. Thus, diabetic patients may experience strong rewarding effects of nicotine that confer enhanced vulnerability to tobacco use.

Supported by: 7-12-BS-135
\end{abstract}

\section{ก 797-P}

The BDNF Polymorphism Is Associated With Depressive Symptom among Newly Diagnosed Diabetic Patients

SOHEE KIM, CHANHEE KYUNG, HAERI BAEK, JIYOON HA, MIN KYUNG KIM, JIWOON KIM, TAEWOONG NOH, SHINAE KANG, JONG SUK PARK, CHUL WOO AHN, KYUNG RAE KIM, YOUNG MI LEE, Seoul, Republic of Korea

The prevalence of depression in patients with DM is twice than in the general population. The brain derived neurotrophic factor (BDNF) plays an important role in neurogenesis and neuroprotection. Some studies have suggested that BDNF plays an antidiabetic role as well as its role in the psychiatric symptoms. The BDNF polymorphism (PM) is known to contribute to psychiatric illness. However, little is known about the impact of the BDNF $\mathrm{PM}$ in DM. We investigated whether the BDNF Val/66/Met polymorphism, glucose status, psychological susceptibility and resilience contribute to development of anxiety (AS) or depression symptom (DS) in newly diagnosed
DM patients. We examined biochemical factors and the BDNF PM in 89 Korean DM subjects. Psychiatric symptoms were investigated by the Hospital Anxiety and Depression Scale (HADS). We analyzed stress that the patients might have from perspectives of psychological resilience and susceptibility by using resilience scale and impact of event scale (IES). 62 out of the 89 were found to be Met carriers (MC). No significant differences were found between $\mathrm{Val} / \mathrm{Val}$ homozygotes (VH) group and $\mathrm{MC}$ group regarding age, sex, $\mathrm{BMI}$ and clinical factors related to glycemic control and lipid profile. HADS anxiety, HADS depression and IES scales such as hyperarousal, avoidance, intrusiveness and sleep problem in $\mathrm{MC}$ group were significantly higher than $\mathrm{VH}$ group (all $\mathrm{P}<0.05)$. HbA1c and $D S$ showed inverse correlation $(r=-0.227$ $\mathrm{P}=0.035)$. Resilience factors such as self efficacy, self confidence, optimism and self control showed inverse correlations and IES factors showed positive correlation with DS. In logistic regression analysis, DS have associations with $\mathrm{HbA1c}(\mathrm{OR}=0.671 ; \mathrm{p}=0.031)$ and BDNF PM (OR=5.413; $\mathrm{p}=0.044)$ whereas hyperarousal was the only variable that is associated with $A S(O R=1.386$; $p=0.024)$. These results suggest that DS is related to presence of the Metallele and lower $\mathrm{HbA1c}$ whereas AS is related to hyperarousal in newly diagnosed DM patients.

Vitamin D Supplementation Improves Depression and Cardiovascular Risk Factors

SUE PENCKOFER, TODD DOYLE, PATRICIA MUMBY, MARY BYRN, MARY ANN EMANUELE, DIANE WALLIS, Maywood, IL, Notre Dame, IN, Downers Grove, IL

Women with type 2 diabetes (T2DM) and co morbid depression are at increased risk of cardiovascular disease (CVD). Epidemiological evidence suggests that low vitamin D levels $(25-\mathrm{OH}-\mathrm{D})$ are associated with increased depressive symptoms and greater cardiovascular risk. The effect of vitamin D supplementation on depression and CVD-related risk factors in T2DM has not been well studied. The Sunshine Study was a single-arm repeated measures trial designed to test the effectiveness of vitamin $\mathrm{D}_{2}$ supplementation on depression (measured by Center for Epidemiologic Studies Depression scale [CES-D] \& Physicians Health Questionnaire [PHQ 9]), blood pressure (BP), and weight outcomes. Women $(\mathrm{N}=46)$ with T2DM and depression were given weekly vitamin $\mathrm{D}_{2}$ supplementation $(50,000 \mathrm{IUS}$ ) for 6 months. Participants were a mean age of 54.6 years $(S D=10.5)$ and $54 \%$ were White. Their baseline HBA1c was $6.8 \%(S D=.82)$ and mean duration of diabetes was 7.8 years $(S D=7.1)$. Results showed that $25-0 \mathrm{H}-\mathrm{D}(\mathrm{ng} / \mathrm{mL})$ levels significantly $(p<.001)$ increased from baseline $(M=18.8, S D=7)$ to 6 -month follow-up (6MFU)(M=37.5, SD=9.5). CES-D scores significantly $(p<.001)$ decreased from baseline ( $M=26.8, S D=7.6)$ to $6 M F U(M=12.2, S D=8.3)$. Similarly, PHQ-9 scores significantly $(p<.001)$ decreased from baseline $(M=11.5, S D=5.4)$ to $6 M F U(M=5.2, S D=3.8)$. Regarding cardiovascular risk factors, BP significantly $(p<.01)$ decreased from baseline $(M=140.4, S D=17.1)$ to $6 \mathrm{MFU}(\mathrm{M}=132.5, \mathrm{SD}=14.6)$ and weight significantly $(\mathrm{p}<.05)$ decreased from baseline ( $M=226.1, S D=59)$ to $6 M F U(M=223.6, S D=59)$. Findings indicated that weekly vitamin $\mathrm{D}_{2}$ supplementation $(50,000 \mathrm{IUs})$ was associated with improvement in depression as well as a reduction in BP and weight among depressed women with T2DM. Randomized, controlled trials are needed to determine the impact of vitamin $\mathrm{D}$ supplementation on depression and major cardiovascular risk factors among women with T2DM.

Supported by: NIH (5P60DK020595); University of Chicago

ก 799-P

Association between High-Sensitivity C-Reactive Protein and Depression in Patients With Type 2 Diabetes

YASUAKI HAYASHINO, YUKO HATAJI, HIROHITO KUWATA, YUSUKE ARAI, SATOSHI MATSUNAGA, SHINTARO OKAMURA, TSUYOSHI MASHITANI, MIYUK FURUYA, MASAKO KITATANI, SHIGEKI IKUSHIMA, SATORU TSUJII, HITOSHI ISHII, TENRI COHORT STUDY GROUP, Nara, Japan, Tenri, Japan

Recent studies suggest a bidirectional association between depression and type 2 diabetes; these conditions may share a common biological pathway. One factor may be chronic inflammation; however, the association between chronic inflammation and depression has not been well evaluated in patients with type 2 diabetes, particularly non-obese patients. This study aimed to evaluate the association between high-sensitivity C-reactive protein (hs-CRP) and depression in patients with diabetes.

We obtained data from 3573 outpatients (average age, 66 years; $38.9 \%$ females) with type 2 diabetes. Based on the diagnostic criteria of the Patient Health Questionnaire [PHO]-9, patients were classified as "not depressed" or with "major depression." Next, we used a multivariable-adjusted logistic regression model to evaluate the association between hs-CRP quartiles and depression 
The prevalence of major depression was 3.4\%; depressed patients were more likely to be female $(p=0.001)$; have high $\mathrm{BMI}$, hs-CRP, and HbA1c levels; more likely to use insulin; exercise less; and smoke more than those without depression. Compared with patients in lowest hs-CRP quartiles, the age- and gender-adjusted relative risk (RR) value for those in the highest hs-CRP quartile was 1.64 (95\% confidence interval $(\mathrm{Cl}), 1.02-2.62$ : $p=0.04$ ). However, this association diminished after adjusting for other possible confounders including BMI. Next, we conducted subgroup analysis based on BMI (low, <25; high, $\geq 25$ ). In patients with high BMI, we observed a significant association $(p=0.036$ ) between the lowest and highest hs-CRP quartiles; the adjusted RR for patients in the highest hs-CRP quartile was $2.18(95 \% \mathrm{Cl}, 1.05-4.51)$. We did not observe a significant association in those with low BMI.

In conclusion, we observed a significant association between high hsCRP and depression in obese, diabetic patients, but we did not observe this association in non-obese, diabetic patients.

\section{ก 800-P}

Exploratory Analysis of Real-Time Measures of Sleep and their Effects on Physical Activity in T2DM

CYNTHIA FRITSCHI, CHANG PARK, ROBIN MERMELSTEIN, LAURIE OUINN, EILEEN G. COLLINS, ANDREW RICHARDSON, Chicago, IL

Curtailed sleep and poor sleep quality have been linked to lower levels of physical activity (PA) in T2DM, but these findings are largely derived from subjective and retrospective studies. The relationship between sleep quality and PA has not been adequately studied using objective measures. The aim of this study was to explore the effects of sleep quality on PA levels in adults with T2DM. Sleep and PA data were gathered using wrist actigraphy (Actiwatch Score ${ }^{\mathrm{IM}}$ ), worn continuously for 7 days to measure physical movement. Subjects completed sleep diaries upon waking and just before bed. We used the Patient Reported Outcomes measurement information System (PROMIS) computerized adaptive testing modules for subjective sleep impairment and sleep disturbance measurement. Sociodemographic and physiological variables were also collected. Preliminary findings were drawn from a diverse sample ( $n=18,50 \%$ African American, $72 \%$ female) of adults (age $57 \pm 4.9 \mathrm{yr}$ ) with T2DM

Baseline findings included A1c $(7.6 \pm 2.3 \%)$, BMI $\left(32.9 \pm 7.5 \mathrm{~kg} / \mathrm{m}^{2}\right)$. PROMIS sleep disturbance $(52.8 \pm 7.3)$, \& sleep impairment $(53.6 \pm 9.6)$ was worse in the T2DM subjects than population norms for the same measures.

We used hierarchical item cluster analyses \& Forest data-mining to derive clusters of sleep variables that most affected PA lactivity counts per minute, averaged daily during wake time). An emerging cluster of sleep variables, including higher self-reported sleep disturbance and impairment, objectively-measured poor sleep efficiency, and shorter sleep duration, were predictive of reduced daily activity. Additionally, partial dependence plots revealed that sleep duration of $\geq 500$ minutes and sleep efficiency $\geq 90 \%$ were threshold levels for predicting significant increases in PA the following day, with no gain in PA beneath the threshold levels. These early findings are among the first to provide objective evidence in support of the role of poor sleep quality as a predictor of reduced PA in adults with T2DM.

Supported by: NIH/NINR (ROONR012219)

\section{ก 801-P Breakfast Skipping, Chronotype and Glycemic Control in Type 2 Diabetes}

SIRIMON REUTRAKUL, STEPHANIE J. CROWLEY, MEGAN M. HOOD, MARY K. MORGAN, MARSHA F. TEODORI, KRISTEN L. KNUTSON, Chicago, IL

Breakfast skipping is associated with obesity and an increased risk for type 2 diabetes. Later chronotypes, individuals who have an "evening preference" (i.e., feeling at their peak later in the day and reporting later sleep times), often skip breakfast. In this study, we explored the relationship among breakfast skipping, chronotype, and glycemic control in type 2 diabetes patients.

Diabetes history and demographics, depressive symptoms, sleep timing, and 24-hour food recall were collected from 194 non-shift working type 2 diabetes patients. Mid-sleep time (midpoint between sleep onset and wake time) on free (non-work) days (MSF) was corrected for sleep debt as suggested by Roenneberg et al (Sleep Med Rev, 2007) and was used as an indicator of chronotype. HbA1C values were obtained from medical records.

Average age (mean $\pm S D$ ) was $58.3 \pm 12.9$ years and $\mathrm{HbA1C}$ was $7.87 \pm 1.64 \%$. MSF was 3:29 \pm 1:46 A.M. Twenty-two subjects (11.3\%) self-reported missing breakfast. Breakfast skippers had a significantly higher HbA1C $(9.02 \pm 2.07$ vs. $7.73 \pm 1.51, p<0.01)$, higher $B M I\left(39.9 \pm 7.2\right.$ vs. $\left.35.1 \pm 8.2 \mathrm{~kg} / \mathrm{m}^{2}, p<0.01\right)$, and later MSF $(4: 33 \pm 2: 18$ vs. $3: 21 \pm 1: 39$ A.M., $p<0.01)$ than individuals who ate breakfast. Later MSF was significantly associated with poorer glycemic control $(r=.34, p<0.001)$. After adjusting for age, sex, race, BMI, diabetes duration, insulin use and depressive symptoms, breakfast skipping was still significantly associated with worse glycemic control $(B=0.136, p<0.01)$. The relationship between breakfast skipping and $\mathrm{HbA1C}$ was partially mediated by chronotype. These results confirm that breakfast skipping is common among late chronotypes. Late chronotype and breakfast skipping both contribute to poor glycemic control. This analysis was limited by only one day of food recall and the small number of non-breakfast eaters. Future studies are needed to confirm these findings and to determine whether behavioral interventions targeting breakfast eating may improve glycemic control in patients with type 2 diabetes.

Supported by: Rush University Medical Center

802-P

ADHD Plus Diabetes Equals Trouble: Diabetes-Related Executive Functioning in Youth

DANNY C. DUKE, JENNIFER K. RAYMOND, LISA SHIMOMAEDA, JONAH HARRIS, KEVIN SHIMOMAEDA, MICHAEL A. HARRIS, Portland, OR

Executive functioning (EF) can be described as an ability to regulate, control, and manage cognitive processes. For example, planning, organizing materials, initiating, monitoring, shift, time management, emotion regulation, inhibit, focus, working memory, and sequential task completion. EF problems are strongly associated with ADHD. Assessing EF in youth with diabetes would inform interventions to improve glycemic control. However, established measures of EF have been only weakly related to adherence and $\mathrm{HbA1c}$. Clinical need led to developing the Diabetes Related Executive Functioning Scale (DREFS)

Youth (Y-DREFS) and parent (P-DREFS) measures were rationally developed by experts in $\mathrm{EF}$ and diabetes to assess diabetes related $\mathrm{EF}$. Measures include 77 items in 11 subscales. The DREFS was administered to 53 caregiver/youth dyads as a pilot study. Mean youth age was 15.3 years $(S D=1.7$ years), $49 \%$ were female, $84 \%$ Caucasian. Mean duration of type 1 diabetes was 6.6 years $(S D=4.3$ ) and mean $\mathrm{HbA1c}$ was $8.7 \%(S D=1.7)$.

Internal reliability was excellent, P-DREFS $(\alpha=.98)$ and Y-DREFS $(\alpha=$ .96) and caregiver/youth agreement was strong $(r=.73, p>.01)$. Convergent validity was shown by correlation of the Behavior Rating Inventory of Executive Functioning (BRIEF) to the P-DREFS $(r=.57, p>.01)$ and the Y-DREFS $(r=.36, p>.01)$, and by correlation of the youth BRIEF (SR) to P-DREFS $(r=.52, p>.01)$ and Y-DREFS $(r=.55, p>.01)$. The Y-DREFS also showed convergent validity by correlating strongly to the Self Administered Diabetes Self Management Profile (SA-DSMP) parent $(r=-.62, p>.01)$ and SA-DSMP youth $(r=-.62, p>.01)$. The P-DREFS showed similar convergent validity by the correlating strongly to the SA-DSMP parent $(r=-.63, p>.01)$, and SA-DSMP youth $(r=-.51, p>.01)$. Predictive validity was indicated by strong correlations of the P-DREFS $(r=.43, p>.01)$ and Y-DREFS $(r=.53, p>$ .01) to HbA1c.

Initial pilot efforts shows the DREFS has strong promise as an important measure for clinical and research applications.

803-P

Targeted Glyoxalase-1 Gene Transfer to Prevent Cerebral Vascular Dysfunction and Cognitive Decline in Rats With Type 1 Diabetes FADHEL ALOMAR, CHUN HONG SHAO, MICHAEL D. BOSKA, MARIANO G. UBERTI, WILLIAM G. MAYHAN, KESHORE R. BIDASEE, Omaha, NE, Shreveport, LA

Reductions in information processing speed, psychomotor function, cognitive flexibility, and visual perception, collectively known as cognitive decline, is a recently appreciated co-morbidity in individuals with Type 1 diabetes (T1D). What we know to date is its severity correlates with the degree of microvascular complications. What remain undefined are the intrinsic molecules that initiate cerebral microangiopathy. Here we show that the reactive di-carbonyl species methylglyoxal (MGO) is a causative agent. Administration of MGO to healthy rats recapitulated impairment cerebral vasoreactivity, cerebral vascular leakage and astrocytes activation seen in streptozotocin (STZ)-induced T1D diabetic rats. Human and rat brain microvascular endothelial cells (ECs) were 7X more susceptible to MGO than smooth muscle cells, astrocytes, neurons and microglia because of their lower steady-state level of the MGO-degrading enzyme glyoxalase-1 (Glo-1). In ECs, MGO sequentially increased cytoplasmic and mitochondria $\mathrm{Ca} 2+$, mitochondrial superoxide (02.) production, reduced expression of tight junction and adhesion proteins, and induce DNA fragmentation. Over-expression of Glo-1 in the cerebral vasculature employing an adenoassociated virus, three days after injection of STZ in rats, lowered MGO 
levels, prevented cognitive decline, impairment in cerebral vasoreactivity, EC loss, cerebral vascular leakage and microglial activation. Targeted Glo1 overexpression also increased perfused capillary density, reduced brain infarct size ischemia/reperfusion injury, and reduced NMDA-mediated vasodilatation of cerebral pial arteriole. This study identifies for the first time, a molecular cause for cognitive impairment in a rat model of T1D and a novel therapeutic strategy to alleviate it.

Supported by: P20RR021937

804-P

Greater Satisfaction and Less Hypoglycemia Among Elderly Patients With Insulin Therapy Using Pens (STEP IT UP Trial)

CHRISTOPHER A. NEWTON, ELIZABETH IVIE, DAWN SMILEY, SAUMETH CARDONA, ROBERTO MEJIA, GUILLERMO E. UMPIERREZ, Atlanta, GA

This crossover randomized study (NCT \#01240200) assessed efficacy, safety, treatment satisfaction and effect on disease burden (physical, psychological and social function) of initiating basal insulin delivered via pen versus syringe (vials) in elderly patients with $\mathrm{T} 2 \mathrm{D}$.

Fifty-six insulin-naïve patients $>60 y$ old with A1c $>7 \%$ treated with oral antidiabetic drugs were randomized to receive glargine for 3 months by pen then 3 additional months by syringe (pen/vial) or to the reverse order (vial/ pen). Insulin was titrated to fasting blood glucose (FBG) of $90-130 \mathrm{mg} / \mathrm{dl}$.

Study outcomes included A1c, FBG, hypoglycemia, Diabetes Treatment Satisfaction Questionnaire status/change scores (DTSQs/DTSQc), Insulin Delivery System Rating Questionnaire (IDSRO), Diabetes Quality of Life Clinical Trial Questionnaire (DOLCTO), Problem Areas In Diabetes scale (PAID) and Elderly Diabetes Burden Scale (EDBS) at 3 and $6 \mathrm{mo}$.

Glycemia improved by 3 mo with pens (A1c: $8.5 \pm 1.3$ to $7.6 \pm 1.0 \%$; FBG: $157 \pm 38$ to $134 \pm 32 \mathrm{mg} / \mathrm{dl}$, both $\mathrm{p}<0.05$ ) and vials (A1c: $8.7 \pm 1.1$ to $7.6 \pm 0.8 \%$; FBG: $159 \pm 47$ to $126 \pm 19 \mathrm{mg} / \mathrm{dl}$, both $p<0.01)$. Satisfaction on DTSQs improved more after $3 \mathrm{mo}$ with pens than vials $(31.5 \pm 4.9$ to $34.2 \pm 2.3$ vs $28.2 \pm 7.0$ to $32.2 \pm 5.2, p<0.05)$. After crossover, satisfaction deteriorated in pen/vial group but improved in vial/pen group (DTS0c: $-2.3 \pm 10.0$ vs $16.4 \pm 2.7, p<0.01$ ) without significant A1c change. Satisfaction was associated with treatment preference, perceived greater clinical efficacy, less diabetes burden and worry on IDRSQ, DQLCTO and EDBS. No change was observed with PAID. Fifteen pen users had 36 hypoglycemic events and 22 syringe users had 72 events, which correlated with patients' perception of more problems with glucose control $(p<0.05)$.

In summary, the use of glargine insulin via pens improves glycemic control in older adults with less hypoglycemia, greater patient satisfaction and perception of decreased burdens with glucose control compared to the use of syringes and vials.

Supported by: sanofi-aventis

805-P

Regional Brain Hyperactivity in Type 2 Diabetes during Cognition THOMAS J. MARDER, VERONICA L. FLORES, WOUTER S. HOOGENBOOM, NICOLAS R. BOLO, ALAN M. JACOBSON, DONALD C. SIMONSON, GAIL MUSEN, Boston, MA, Mineola, NY

Patients with T2DM are at increased risk for Alzheimer's disease (AD). In $A D$ risk groups, functional brain imaging has revealed both reduced deactivation of the default mode network (DMN) and hyperactivation of task-relevant regions during cognition. These changes occur well before clinical manifestations of cognitive decline and may be early biomarkers for $\mathrm{AD}$. Using $\mathrm{AMRl}$, we investigated whether this pattern is observed in T2DM during a recognition task. We examined 19 T2DM patients not on TZDs or metformin (11 M, mean $( \pm S D)$ age $56 \pm 6$ yrs, education (ed) $15 \pm 2.3$ yrs, BMI $30.4 \pm 4.4 \mathrm{~kg} / \mathrm{m}^{2}$, HbA1c $7.4 \pm 1.9 \%$, fasting plasma glucose (FPG) $135 \pm 67 \mathrm{mg} / \mathrm{dl}$, fasting insulin $120 \pm 96 \mathrm{pmol} / \mathrm{I}$, DM duration $9.6 \pm 6.6 \mathrm{yrs}, 10$ $107 \pm 14)$ and 29 control subjects (19 M, age $53 \pm 6$ yrs, ed $16 \pm 2.5 \mathrm{yrs}, \mathrm{BMI}$ $28.6 \pm 4.8 \mathrm{~kg} / \mathrm{m}^{2}, \mathrm{HbA1c} 5.6 \pm 0.3 \%, \mathrm{FPG} 82 \pm 7 \mathrm{mg} / \mathrm{dl}$, fasting insulin $78 \pm 69$ $\mathrm{pmol} / \mathrm{I}, \mathrm{IO} 113 \pm 12$ ). Recognition performance was $69 \%$ in both groups. Because I0, memory and executive function were lower in T2DM patients $(p<0.05)$, we controlled for these factors in the analyses. T2DM showed increased activation during recognition in the right anterior cingulate, right medial frontal gyrus, and right caudate, compared to controls, and did not deactivate the DMN $(p<0.05)$ after controlling for cognitive variables, HOMA-IR, and the AD risk gene APOE\&4. Of the regions with greater activation in T2DM compared with controls, the caudate was positively correlated with $\mathrm{HbA1c}(r=0.45, p=0.05)$, suggesting that activation in these regions is greater in T2DM in worse glucose control. The pattern of deficient deactivation of the DMN coupled with hyperactivation in task-relevant regions is consistent with observations of other $A D$ risk populations, suggesting that greater brain activity is needed to maintain cognitive function in T2DM compared to controls. This pattern may warn of future clinically evident cognitive dysfunction in T2DM.

Supported by: NIH (AG034165-A1); Joslin Diabetes Center (P30-DK-36836); Herbert Graetz Fund

\section{Cross-Country Psychosocial Benchmarking Indicators Identified fo Family Members Living With Persons With Diabetes in the DAWN2} Study

KATHARINA KOVACS BURNS, NORBERT HERMANNS, RICHARD I.G. HOLT, SANJAY KALRA, ANTONIO NICOLUCCI, FRANS POUWER, SØREN SKOVLUND, JOHAN WENS, INGRID WILLAING, MARK F. PEYROT, ON BEHALF OF THE DAWN2 STUDY GRoUP, Edmonton, AB, Canada, Bad Mergentheim, Germany, Southampton, United Kingdom, Karnal, India, Santa Maria Imbaro, Italy, Tilburg, Netherlands, Bagsvaerd, Denmark, Antwerp, Belgium, Gentofte, Denmark, Baltimore, MD

In the second Diabetes Attitudes, Wishes and Needs (DAWN2) study, 2057 family members (FM) of persons with diabetes (PWD) across 17 countries within 4 continents were surveyed for their perceptions living with a PWD. About $120 \mathrm{FM}$ in each country were asked to complete psychometrically comparable surveys to PWD, transcribed to local languages. Adapted questions were used, some from instruments such as the WHO-Quality of Life, WHO-5 Well-being Index, Problem Areas in Diabetes Scale, Diabetes Impact on Life Dimensions, Diabetes Empowerment Scale, and Supports Scale, and others developed for this survey.

Results were statistically significant $(p<0.001)$ for the cross-country comparisons of the various psychosocial indicators. While over half of all FM respondents indicated that their quality of life was good/very good (cross-country scores ranged from a low of $22.5 \%$ to a high of $76.0 \%$ ). $45.6 \%$ of respondents felt their distress related to thinking about the person with diabetes was high (country variation range, 21.7-76.7\%), and $61.3 \%$ worried about the risk of hypoglycemic events (range, 31.5$86.4 \%)$. As well, a notable burden of diabetes on the family was perceived by over one-third of respondents, with a large cross-country variation (range, 10.0-61.7\%). Negative impact of the disease was felt by $41.3 \%$ of all FM (range, 36.7-42.9\%) with highest on emotional well-being $(44.6 \%$; range, $31.8-63.0 \%)$ and much less with relationship aspects $(19.8 \%$; range, $10.8-34.5 \%$ ). Although one in three $\mathrm{FM}$ (range, 17.5-53\%) do not know how to best help the PWD they live with, 39.4\% (range, 15.5-61.7) said they would like to be somewhat/much more involved in the diabetes care and almost half (range, 25.8-69.2) in helping PWD with feelings about diabetes.

The results will provide a strong benchmarking baseline for FM worries, burden and other psychosocial indicators, which will also help identify what supports and education FM need to help themselves and the PWD they live with.

807-P

Youth-Perceived Burden of Type 1 Diabetes (T1D): Problem Areas in Diabetes-Pediatric Survey (PAID-Peds)

JESSICA T. MARKOWITZ, DEBORAH A. BUTLER, LISA K. VOLKENING, LORI M. LAFFEL, Boston, MA

Validated versions of the Problem Areas in Diabetes Survey (PAID) are used to measure perceived burden of T1D in parents, adults, and teens. We created and validated the PAID-Peds to assess perceived burden of T1D in youth. The PAID-Peds is a 20 -item self-report survey with a 5 -item response scale; scores range from 0-100 (higher score=greater burden). In 93 youth, ages 8-17, we assessed internal consistency (Cronbach's $\alpha$ ), testretest reliability, and construct validity against measures of T1D-specific family conflict, mood, and quality of life (QOL; generic and diabetes-specific). Youth ( $49 \%$ male, $86 \%$ pump Rx) were $12.7 \pm 2.7$ yrs old, had T1D for $5.9 \pm 3.6$ yrs, and had mean A1c $8.0 \pm 0.9 \%$. PAID-Peds scores ranged from 0-95 $($ mean $=29.5 \pm 23.4)$. The PAID-Peds showed excellent internal consistency $(\alpha=0.93)$; factor analysis confirmed 1 factor. Test-retest reliability, assessed $8 \pm 2$ days between responses, was acceptable $(r=0.71, p<0.0001)$. PAID-Peds scores were not associated with attained age, age at T1D Dx, duration, pump $\mathrm{Rx}$, or A1c. PAID-Peds scores correlated positively with T1D-specific family conflict, youth depression, negative affect around BG monitoring, state/ trait anxiety, and parent-reported perceived T1D burden. PAID-Peds scores correlated negatively with generic and T1D-specific 00L (see table). The PAID-Peds is a valid and reliable measure of youth's perceived T1D burden that offers both clinical and research utility. 
PSYCHOSOCIAL, BEHAVIORAL MEDICINE

Table: Construct validity of PAID-Peds

\begin{tabular}{|c|c|c|}
\hline & $\mathrm{r}$ & $\mathrm{p}$ \\
\hline $\begin{array}{l}\text { Diabetes Family Conflict Scale } \\
\text {.....Child report } \\
\text { f...Parent report }\end{array}$ & $\begin{array}{l}0.35 \\
0.22\end{array}$ & $\begin{array}{l}0.0005 \\
0.03\end{array}$ \\
\hline Center for Epidemiologic Studies Depression Scale & 0.46 & $<0.0001$ \\
\hline $\begin{array}{l}\text { BG Monitoring Communication Survey } \\
\text { ….Child report } \\
\text {....Parent report }\end{array}$ & $\begin{array}{l}0.64 \\
0.31 \\
\end{array}$ & $\begin{array}{c}<0.0001 \\
0.003\end{array}$ \\
\hline $\begin{array}{l}\text { State Trait Anxiety Inventory (STAI) } \\
\text { …State anxiety } \\
\text { …Trait Anxiety } \\
\text { …Parent-proxy trait version }\end{array}$ & $\begin{array}{l}0.23 \\
0.46 \\
0.30\end{array}$ & $\begin{array}{c}0.03 \\
<0.0001 \\
0.004\end{array}$ \\
\hline Parent PAID (PAID-PR) & 0.33 & 0.001 \\
\hline $\begin{array}{l}\text { PEDS QL Generic Core Scales } \\
\text {...... Child report } \\
\text {....Parent-proxy report }\end{array}$ & $\begin{array}{l}-0.52 \\
-0.39 \\
\end{array}$ & $\begin{array}{l}<0.0001 \\
<0.0001\end{array}$ \\
\hline $\begin{array}{l}\text { Peds QL Diabetes Module } \\
\text {.....Child report } \\
\text {....Parent-proxy report } \\
\end{array}$ & $\begin{array}{l}-0.55 \\
-0.27 \\
\end{array}$ & $\begin{array}{c}<0.0001 \\
0.009\end{array}$ \\
\hline
\end{tabular}

808-P

Depression in Adults With Type 1 Diabetes Participating in the T1D Exchange Clinic Registry

PAULA M. TRIEF, DONGYUAN XING, JULIE KITTELSRUD, BETH A. OLSON, LAURA YOUNG, ANNE L. PETERS, KELLEE M. MILLER, ROY BECK, RUTH S. WEINSTOCK, Syracuse, NY, Tampa, FL, Sioux Falls, SD, Minneapolis, MN, Chapel Hill, NC, Los Angeles, CA

To assess the frequency of depression measured with the PHQ-8 in adults with type 1 diabetes, an analysis was conducted of 3,647 adults participating in the T1D Exchange clinic registry (median age 33.0 yrs, median duration of T1D $16.0 \mathrm{yrs}, 55 \%$ female, 89\% non-Hispanic white). Characteristics and clinical outcomes among those with and without depression were compared using logistic and linear regression models. A participant was classified as having clinically significant depression symptoms if the total score on the $\mathrm{PH} 0-8$ was $\geq 10$.

Among the 3,647 participants, $357(9.8 \%)$ were classified as having significant depression symptoms. Participants classified as depressed were more likely to be female, Hispanic, have a lower household income level and a lower education level, exercise less often, miss insulin doses, live alone, and have $\geq 1$ complication (neuropathy, nephropathy, treatment for retinopathy, or cardiovascular/ cerebrovascular disease, $\mathrm{p}<0.01$ for all comparisons). Mean $\mathrm{HbA1c}$ was higher among those with depression symptoms compared with those classified as not depressed $(8.5 \pm 1.7 \%$ vs. $7.7 \pm 1.4 \%, p<0.001)$. Notably there was a higher occurrence of $\geq 1$ DKA event $(10.9 \%$ vs. $4.1 \%$, $p<0.001)$ and $\geq 1$ severe hypoglycemic event $(17.9 \%$ vs. $9.1 \%, p<0.001)$ in the past 3 months among depressed participants.

In the T1D Exchange clinic registry, adults with depression have worse clinical outcomes than those not depressed. Whether identification and treatment of depression will improve diabetes outcomes or reduce acute or chronic diabetes complications requires further study. Depression is common in T1D and better identification and treatment of this co-morbid condition is urgently needed.

Supported by: Leona M. and Harry B. Helmsley Charitable Trust

$\Delta$

809-P

Diabetes Family Impact Scale (DFI-S): Psychometric Properties Assessed in Parents of Youth With Type 1 Diabetes (T1D)

MICHELLE L. KATZ, LISA K. VOLKENING, EMILY A. FISHER, LORI M. LAFFEL, BOSton. MA

Modern T1D management impacts families and may negatively affect youth quality of life ( 0 oL). We created a novel parent self-report scale to describe and quantify T1D impact on families and evaluate its contribution to youth $\mathrm{QoL}$.

Derived from research literature and input from pediatric diabetes MDs, nurse educators, psychologists, and parents, the DFI-S measures T1D impacts in the domains of "School", "Work", "Finance", and "Family Wellbeing". We assessed its internal consistency (Cronbach's $\alpha$ ) and convergent validity (compared with parent-perceived diabetes burden (PAID-P) and Life Events Checklist (LEC)) in 148 parents (84\% mothers; 92\% white) of T1D youth. Parents also completed surveys about their child's generic and diabetes-specific QoL (parent proxy PedsQLs). The 14 item DFI-S has 4 response options (Almost Never, Sometimes, Often, Almost Always). Total scores range from 0-100 (higher scores=more impact).

Offspring ( $50 \%$ male) of respondents had an age of $12.9 \pm 3.3 \mathrm{yrs}$ (range $6-18$ ) and T1D duration of $6.2 \pm 3.6$ yrs. Mean A1c was $8.4 \pm 1.3 \%$; $66 \%$ were on pump Rx. DFI-S score was $18.9 \pm 15.2$ (range $0-74$ ). Only $6 \%$ of parents endorsed no impact. Internal consistency was good $(\alpha=0.85)$. The DFI-S correlated positively with the PAID-P $(r=0.4, p<.0001)$ and LEC $(r=0.3$, $\mathrm{p}=.0001)$. The DFI-S correlated negatively with child age $(r=-0.2, p=.03)$ but had no associations with T1D duration, age at T1D onset, pump Rx, or A1c. DFI-S correlated negatively with both generic and diabetes-specific youth OoL ( $r=-0.5$ to $-0.6, p<.0001)$. After adjusting for youth age, sex, T1D duration, pump Rx, A1c, parents' diabetes burden, and life events, the DFI-S uniquely predicted both generic and diabetes-specific youth OoL, explaining 14-17\% of variance $(p<.0001)$

In the absence of automated T1D management tools, it is likely that families of T1D youth will continue to experience substantial impact. The DFI-S validly measures negative impacts on families and uniquely describes risk to youth QoL.

Supported by: NIH/NIDDK (1K12DK094721-01)

810-P

Altered Baseline Brain Activity in Type 2 Diabetes: A Resting-State fMRI Study

WENOING XIA, YUE YANG, YI ZHOU, PIN WANG, QIONG WEI, YAN HUANG, RONGRONG CAI, SHAOHUA WANG, Nanjing, China

Purposes: Type 2 diabetes mellitus (T2DM) has been associated with cognitive impairment, such as dementia and Alzheimer's disease. Our study aims to investigate whether altered baseline brain activity exists in T2DM patients.

Methods: T2DM patients ( $\mathrm{n}=28$ ) were compared with nondiabetic agematched control subjects $(n=29)$ using resting-state functional magnetic resonance imaging (rs-fMRI). Amplitude of low-frequency fluctuations (ALFF) of fMRI signal was computed to measure the spontaneous neuronal activity.

Results: Compared with healthy controls, T2DM patients had significantly decreased ALFF values in bilateral middle temporal gyrus (MTG), left fusiform gyrus, left middle occipital gyrus, right inferior occipital gyrus; and increased ALFF values in both cortical and subcortical regions, including the bilateral cerebellum posterior lobe, right cerebellum culmen, left anterior cingulate gyrus, left precuneus and insula lobe. Moreover, the ALFF values in the middle temporal gyrus was found to correlated inversely with both $\mathrm{HbA} 1 \mathrm{c}(\mathrm{r}=$ $-0.420, p=0.026)$ and the score of Trail Making Test-B (TMT-B) $(r=-0.451, p=$ 0.016 ) among the T2DM patients.

Conclusion: The present study provides evidence that T2DM patients have altered ALFF in many brain regions, which links with poor neurocognitive performances and the severity of consistent hyperglycemic state. Those findings may contribute to further understanding of neurological pathophysiology underlying T2DM

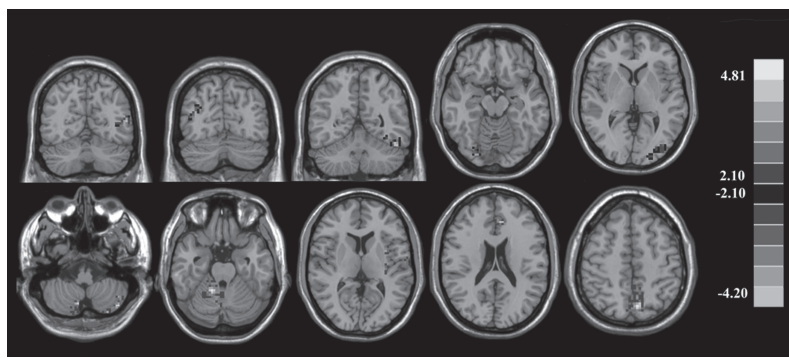

811-P

Cross-Country Benchmarking of Diabetes Psychosocial Outcomes for People With Diabetes in the DAWN2 Study

ANTONIO NICOLUCCI, KATHARINA KOVACS BURNS, MARCO COMASCHI, NORBERT HERMANNS, RICHARD I.G. HOLT, FRANS POUWER, SØREN SKOVLUND, HEATHER L. STUCKEY, ILHAN TARKUN, MICHAEL VALLIS, JOHAN WENS, MARK F. PEYROT, ON BEHALF OF THE DAWN2 STUDY GROUP, Imbaro, Italy, Edmonton, $A B$, Canada, Rapallo, Italy, Bad Mergentheim, Germany, Southampton, United Kingdom, Tilburg, Netherlands, Bagsvaerd, Denmark, Hershey, PA, Kocaeli, Turkey, Halifax, NS, Canada, Antwerp, Belgium, Baltimore, MD

To identify country-level peaks and shortfalls in diabetes outcomes, the second Diabetes Attitudes, Wishes and Needs (DAWN2) study assessed outcomes in 17 countries across 4 continents. In each country, a sample 
of 500 people with diabetes, stratified by diabetes type and treatment, completed a psychometrically and cross-culturally validated questionnaire in local languages. The survey incorporated the newly developed Diabetes Impact on Life Dimensions; WHO-5 Well-being Index and WHO-Quality of Life; Problem Areas in Diabetes (PAID-5); and Summary of Diabetes SelfCare Activities (SDSCA).

Participants were 1368 adults with T1DM and 7228 with T2DM. The proportion with likely depression (WHO- 5 score $\leq 28$ ) was $15 \%$, ranging from $6.6 \%$ in Denmark to $23.7 \%$ in Algeria. High diabetes distress (PAID-5 $\geq 40$ ) was reported by $29.6 \%$ of participants, ranging from $13 \%$ in Denmark and the Netherlands to $>40 \%$ in Italy, Algeria and Turkey. Quality of life was rated "poor"/"very poor" by $13.9 \%$ of participants, with wide betweencountry variation (from $7.6 \%$ in Denmark to $29.3 \%$ in Japan). Diabetes had a negative impact on all domains investigated, ranging from $20.4 \%$ on relationship with family/friends to $61.1 \%$ on physical health. Negative financial impact was reported by $40 \%$ (range, $<30 \%$ in Northern Europe to $>60 \%$ in Eastern Europe). Respondents were most likely to follow self-care advice for medication and diet and least likely to follow advice on glucose monitoring and foot examination. There were between-country differences in self-care activities: participants in USA, Algeria and Turkey were below the median for physical activity, while those in Mexico, Algeria, Turkey, India and Japan were less likely to undertake blood glucose self-monitoring, and foot examination was less likely in Turkey, India, China and Japan.

Cross-country benchmarking can help identify unmet needs and best practices that can be used to drive changes to improve outcomes for people with diabetes.

812-P

Use of $\mathrm{Pa}^{\circledR}{ }^{\circledR}$, a Simple 3-Day Basal/Bolus Insulin Delivery Device, Reduces Barriers to Insulin Therapy in Patients With Type 2 Diabetes

NORBERT HERMANNS, LESLIE LILLY, JULIA K. MADER, FELIX ABERER, JOERG PACHATZ, STEFAN KORSATKO, JAY WARNER, THOMAS PIEBER, Bad Mergentheim, Germany, Marlborough, MA, Graz, Austria

$\mathrm{PaO}$ (CeOur SA) is a simple to use patch-on device which provides set basal rates and bolus insulin on demand. $\mathrm{PaO}$ was designed to minimize barriers to insulin therapy. In addition to feasibility of use, safety and efficacy (reported elsewhere), this study analyzed the impact of the use of $\mathrm{PaO}$ on patient reported outcomes (PRO) including; barriers against insulin treatment, diabetes related distress and negative attitudes towards insulin therapy in twenty patients with type 2 diabetes (T2D) on a stable multiple daily injections (MDI) insulin regimen. This single center, open label, single arm study was comprised of three 2-week periods; baseline (MDI), transition from $\mathrm{MDI}$ to $\mathrm{PaO}$, and $\mathrm{PaO}$ treatment. Three validated questionnaires were completed at the end of the baseline and $\mathrm{PaO}$ treatment periods; Barriers to Insulin Treatment - Questionnaire (BIT), Problem Areas In Diabetes -Scale (PAID) and Insulin Treatment Appraisal Scale (ITAS). Nineteen patients (age $59 \pm 5$ y, T2D duration $15 \pm 7$ y, 21\% female, A1C $7.7 \pm 0.7 \%$ ) completed the questionnaires at the two measurement points. There was a strong and significant effect of $\mathrm{PaO}$ in the mean BIT total score (2.5 to 2.1, difference $(D)=0.4 \pm 0.6 ; p=.01$, effect size $(d)=0.70$ ). Patients perceived less hardship from insulin therapy (overall improvement $\mathrm{d}=0.35$ ), less stigmatization by insulin injection (overall improvement $\mathrm{d}=0.28$ ) and less fear of hypoglycemia (overall reduction $\mathrm{d}=0.29$ ). Diabetes related distress was slightly reduced (PAID 21.7 to 21.0, $D=0.7 \pm 6.7, p=0.79, d=10$ ). A non-significant reduction was also seen in the mean ITAS score (42.80 to $40.80, D=2.0 \pm 6.5, p=.20$, $d=31$ ). The study is limited by both the uncontrolled design and small sample size, however, the results and the moderate to large effect sizes suggest that the use of $\mathrm{PaO}$ has beneficial and clinically relevant effects to overcome barriers to and negative appraisal of insulin treatment, without increasing other diabetes related distress.

Supported by: CeQUr Corporation

813-P

Restful Yoga Improves Metabolic Risk Factors: Restorative Yoga vs. Stretching for the Metabolic Syndrome (PRYSMS) Study

ALKA M. KANAYA, ROGER COLE, SARAH B. PAWLOWSKY, MARY LOU CARRIONPETERSEN, ELIZABETH L. BARRETT-CONNOR, MARIA R. ARANETA, San Francisco, CA, Del Mar, CA, San Diego, CA

Intensive lifestyle change prevents type 2 diabetes, but lifestyle change is difficult to sustain. We tested two novel behavioral interventions for ameliorating diabetes risk factors.

We conducted a 48-week randomized trial comparing restorative yoga vs. active stretching at two clinical sites. Eligible participants were between 21-65 years, underactive, and met metabolic syndrome criteria. We excluded those with diabetes, taking niacin, fibrates, or hormones. Participants received a tapering series of 90-minute group classes in the 24-week intervention period and monthly classes during the maintenance phase (weeks 25-48). Home practice consisted of 30 minutes/day at least 3 times/week. Both groups received standard lifestyle counseling.

Mean age of the 180 randomized participants was $55 \pm 7$ years, $73 \%$ were women and $35 \%$ ethnic minorities: BMl was $34.4 \pm 6.9 \mathrm{~kg} / \mathrm{m}^{2}$ and waist circumference was $108.1 \pm 13.4 \mathrm{~cm}$. A total of $135(75 \%)$ participants completed the trial $(79 \%$ yoga vs. $71 \%$ stretch, $p=0.20)$. The table shows changes in metabolic factors at 6 and 12 months. Both groups significantly improved many metabolic parameters, but the difference between groups was significant only for fasting glucose at 12 months $(-6.4 \mathrm{mg} / \mathrm{dl}$ in yoga vs. -0.5 in stretch; $p=.002$ ).

Restorative yoga may be a novel method to improve health in patients with the metabolic syndrome.

\begin{tabular}{|c|c|c|c|c|c|c|}
\hline & \multicolumn{3}{|c|}{ Restorative Yoga, n (95\% Cl) } & \multicolumn{3}{|c|}{ Stretching, n (95\% Cl) } \\
\hline & Baseline & $\begin{array}{c}\text { Change at } \\
6 \mathrm{mo}\end{array}$ & $\begin{array}{c}\text { Change at } \\
12 \mathrm{mo}\end{array}$ & Baseline & $\begin{array}{c}\text { Change at } \\
6 \mathrm{mo}\end{array}$ & $\begin{array}{c}\text { Change at } \\
12 \mathrm{mo}\end{array}$ \\
\hline Weight, kg & $97.2 \pm 19.1$ & $\begin{array}{c}-1.3 \\
(-1.9,-0.7)^{*}\end{array}$ & $\begin{array}{c}-1.7 \\
(-2.6,0.7)^{*}\end{array}$ & $90.3 \pm 19.1$ & $\begin{array}{c}-0.7 \\
(-1.4,-0.1)^{*}\end{array}$ & $\begin{array}{c}-0.7 \\
(-1.7,0.3)\end{array}$ \\
\hline Visceral fat area, cm2 & $192 \pm 68$ & $\begin{array}{c}-2 \\
(-10,5)\end{array}$ & $\begin{array}{c}-1 \\
(-9,7) \\
\end{array}$ & $178 \pm 74$ & $\begin{array}{c}-3 \\
(-11,4) \\
\end{array}$ & $\begin{array}{c}-4 \\
(-12,4) \\
\end{array}$ \\
\hline Waist circ., cm & $110.6 \pm 12.8$ & $\begin{array}{c}-1.7 \\
(-2.6,-0.7)^{*}\end{array}$ & $\begin{array}{c}-2.1 \\
(-3.1 .1,-1.0)^{*}\end{array}$ & $105.4 \pm 13.5$ & $\begin{array}{c}-0.8 \\
(-1.9,0.2)\end{array}$ & $\begin{array}{c}-1.1 \\
(-2.2,-0.1)^{*}\end{array}$ \\
\hline Fasting glucose, mg/dl & $104 \pm 12$ & $\begin{array}{c}-0.3 \\
(-2.5,2.0) \\
\end{array}$ & $\begin{array}{c}-6.4 \\
(-8.9,-3.8)^{*}+\end{array}$ & $103 \pm 11$ & $\begin{array}{c}1.3 \\
(-1.1,3.8) \\
\end{array}$ & $\begin{array}{c}-0.5 \\
(-3.4,2.4)\end{array}$ \\
\hline 2-hour OGTT glucose, mg/dl & $133 \pm 48$ & $\begin{array}{c}-1.1 \\
(-8.1,6.0)\end{array}$ & $\begin{array}{c}-6.1 \\
(-13.6,1.4) \\
\end{array}$ & $127 \pm 48$ & $\begin{array}{c}-2.8 \\
(-10.8,5.2) \\
\end{array}$ & $\begin{array}{c}-6.3 \\
(-14.9,2.3) \\
\end{array}$ \\
\hline HbA1c, \% & $5.9 \pm 0.4$ & $\begin{array}{c}-0.07 \\
(-0.13,-0.01)^{*}\end{array}$ & $\begin{array}{c}-0.05 \\
(-0.11,0.00)\end{array}$ & $5.9 \pm 0.4$ & $\begin{array}{c}-0.05 \\
(-0.12,0.01)\end{array}$ & $\begin{array}{c}-0.04 \\
(-0.1,0.02)\end{array}$ \\
\hline Fasting insulin, $\mu \mathrm{U} / \mathrm{ml}$ & $27 \pm 12$ & $\begin{array}{c}-0.01 \\
(-2.2,2.2)\end{array}$ & $\begin{array}{c}-2.4 \\
(-4.5,-0.3)^{*}\end{array}$ & $28 \pm 22$ & $\begin{array}{c}0.06 \\
(-2.3,2.4)\end{array}$ & $\begin{array}{c}-1.2 \\
(-3.5,1.0)\end{array}$ \\
\hline Triglycerides, mg/dl & $161 \pm 55$ & $\begin{array}{c}-6 \\
(-17,6)\end{array}$ & $\begin{array}{c}-3 \\
(-17,11)\end{array}$ & $172 \pm 82$ & $\begin{array}{c}-16 \\
(-28,-4)^{*}\end{array}$ & $\begin{array}{c}-9 \\
(-16,14)\end{array}$ \\
\hline HDL, mg/dl & $49 \pm 10$ & $\begin{array}{c}1.8 \\
(0.4,3.1)^{*}\end{array}$ & $\begin{array}{c}0.8 \\
(-0.7,2.3)\end{array}$ & $48 \pm 11$ & $\begin{array}{c}1.3 \\
(-0.1,2.8)\end{array}$ & $\begin{array}{c}1.4 \\
(-0.3,3.0)\end{array}$ \\
\hline Systolic BP, mmHg & $124 \pm 14$ & $\begin{array}{c}1 \\
(-2,3)\end{array}$ & $\begin{array}{c}1 \\
(-1,4)\end{array}$ & $124 \pm 14$ & $\begin{array}{c}-1 \\
(-3,2)\end{array}$ & $-1(-4,2)$ \\
\hline
\end{tabular}

${ }^{*}$ within-group difference, $\mathrm{p}<0.05$ compared to baseline

$\dagger$ between-group difference, $p<0.05$

Supported by: NIH/NCCAM (5R01AT004569-02)

814-P

Designing a Peer Mentoring Program to Improve Adherence in Adolescents and Young Adults With Type 1 Diabetes (T1D): Focus Group Findings

ELIZABETH PYATAK, PAOLA A. SEQUEIRA, JAMIE R. WOOD, ANNE L. PETERS, LUCY MONTOYA, YANG LU, Los Angeles, CA

Peer mentoring (PM) has shown promise as a cost-effective approach to improve health in populations with chronic conditions. However, PM has not been evaluated as a strategy to enhance adherence in youth with T1D. As part of a study assessing the feasibility of a PM intervention, program development focus groups and interviews were conducted with young adults/prospective mentors (19-25 yrs; $n=11)$, adolescents/prospective mentees (13-18 yrs; $n=4)$, and adolescents' parents $(n=5)$.

Regarding program structure, respondents favored a combination of group and one-on-one meetings on an ongoing basis. They preferred group meetings to be structured around social activities (e.g. dining out, bowling), organized by a facilitator knowledgeable about diabetes, but incorporating minimal didactic or structured content

Respondents preferred pairings with mentors in a different life stage than mentees (e.g. high school, college, post-college). They felt that while mentors should have adequate control of diabetes, it was also desirable for them to have undergone challenges in their diabetes care. Prospective mentors wanted opportunities for ongoing training and debriefing.

Content areas of interest included social and emotional challenges such as feeling different from peers; applying dietary recommendations in realworld settings; checking blood glucose as recommended; and advice on major transitions (e.g. moving away from home, transferring to adult care).

Logistical challenges included scheduling, transportation, and geographic constraints. For example, some respondents planned to move away for 
college. For these reasons, an online component to the program was suggested to supplement in-person contact.

Overall, participants viewed the PM concept favorably and had few concerns about its implementation. Further development and pilot testing of a PM program will incorporate their input regarding program structure, content, and logistics.

Supported by: NIDDK (1R03DK094513-01)

815-P

Correlates of Patient Perception of Physicians' Level of Communicative Support

BASILIO PINTAUDI, SANDRO GENTILE, GIUSEPPE LUCISANO, GIAMPIERO MARRA, FABIO PELLEGRINI, MARIA CHIARA ROSSI, SOREN EIK SKOVLUND, GIACOMO VESPASIANI, ANTONIO NICOLUCCI, BENCH-D AMD STUDY GROUP, Santa Maria Imbaro, Italy, Naples, Italy, Rome, Italy, Copenhagen, Denmark, San Benedetto del Tronto, Italy

The Chronic Care Model emphasizes practical support and communication as means of improving the clinical outcomes of patients with diabetes. We evaluated correlates of patient perception of physicians' level of communicative support, using the Health Care Climate Questionnaire (HCCO). The score ranges from 0 to 100; higher scores indicate higher levels of communicative support. The study was conducted in 28 diabetes outpatient clinics in Italy in the context of the DAWN-2 initiatives. In each center, a random sample of patients was enrolled. Additional questionnaires used were: the Diabetes Empowerment Scale (DES), the WHO-5 well-being index, the Problem Areas in Diabetes (PAID-5), the Patients Assessment of Chronic IIIness Care (PACIC), the Patient Support (PS) questionnaire, the Diabetes Self-care Activities (DSCA) questionnaire, and the Global Satisfaction for Diabetes Treatment (GSDT). Clinical data were extracted from computerized medical records. Overall, 2434 patients were evaluated (mean age $65.0 \pm 10.2$ years, diabetes duration $13.8 \pm 15.2$ years, $59.9 \%$ males, $48.6 \%$ treated with $\mathrm{OHA}, 25.3 \%$ treated with insulin+OHA, 24.3\% treated with insulin). When compared with patients in the other quartiles of the $\mathrm{HCCO}$ score, patients in the lower quartile showed significantly lower scores for WH0-5, PACIC, DES, PS, DSCA, and GSDT $(p<0.001$ for all the scales). At multivariate analysis, adjusted for socio-demographic and clinical characteristics, the risk of presenting a $\mathrm{HCCO}$ score in the lowest quartile decreased for increasing values (by 5 score points) of GSDT (OR $0.65 ; 95 \% \mathrm{Cl}$ 0.56-0.77), PACIC (OR 0.56; 95\%CI 0.52-0.60), DES (OR 0.86; 95\%CI 0.780.95), WHO-5 (OR 0.96; 95\% CI 0.93-0.98) and PAID-5 (OR 0.96; 95\%CI 0.940.99). In conclusion, higher levels of communicative support are associated with higher levels of psychological well-being, satisfaction, perceived support and empowerment, higher compliance with self-care activities, and lower perception of diabetes burden.

Supported by: Novo Nordisk Italy

816-P

Barriers to Participation in a Community-Based Lifestyle Intervention Programme to Prevent Type 2 Diabetes following Gestational Diabetes Mellitus

JENNIFER J. INFANTI, ANGELA O'DEA, PADDY GILLESPIE, CIARAN O'NEILL, LIAM G. GLYNN, BRIAN E. MCGUIRE, FIDELMA P. DUNNE, Galway, Ireland

Gestational diabetes mellitus (GDM) is increasing and progression to type 2 diabetes is increased seven-fold following GDM. We have designed and are recruiting into a community-based randomised controlled trial (RCT) of an intensive lifestyle intervention compared to standard care for delaying diabetes onset following GDM. This paper examines the barriers to participation in women eligible for recruitment but who declined to participate. 410 women with prior GDM were invited to participate, 89 consented, 156 refused, and the remainder were not contactable. Clinical, anthropometric and demographic details are available on all women $(n=410)$ from their post-partum clinic visit following GDM. Stated barriers to participation are also available from the 156 non-consenters. The barriers cited by women can be grouped as follows: access/transport (distance, location, expense / lack of transportation) [n=75]; lifestyle (too busy, intensive work schedule, caring for parents, learning English, planning pregnancy) $[n=49]$; parental commitments [ $n=48]$; health-related barriers (poor health, not concerned about health or diabetes risks, taking action to improve health) [n=29]; research programme deterrents (research fatigue, test procedures, programme times/content) [n=26]; lack of social support $[\mathrm{n}=9]$. To improve recruitment into similar programmes, researchers should consider offering home-based assessments; providing subsidies for child care/transportation; ensuring a variety of programme times; including e-health solutions and testimonials from women who have successfully completed the programme. It is important also to focus on updating and reinforcing knowledge about the future health implications of GDM, as well as the use of cognitive behavioural strategies to progress women who are still "pre-contemplators" in regard to lifestyle modification to the stage of contemplation and action.

Supported by: Irish Health Research Board

817-P

Differences in Social Relations between Persons With Type 2 Diabetes and the General Population

NANA F. HEMPLER, OLA EKHOLM, INGRID WILLAING, Gentofte, Denmark

Good social relations, such as social support and network contacts, are associated with better self-management and fewer psychosocial problems in persons with type 2 diabetes. Persons with chronic disease may be particularly prone to poor social relations, as lack of social support is a risk factor for morbidity. The aim of this study was to investigate whether persons with type 2 diabetes have poorer social relations than the general population.

We conducted a cross-sectional survey in three settings; a specialist diabetes clinic (SDC) $(n=1,084)$, a web panel (WP) consisting of persons with type 2 diabetes $(n=1,491)$ and a sample from the 2010 Danish Health and Morbidity Survey, representative of the general population $(n=15,165)$. We compared social relations using multivariate logistic regression.

Compared to the general population, persons with type 2 diabetes more often lived without a partner (SDC, OR 1.75, 95\% CI 1.49, 2.06; WP, OR 1.64, $95 \%$ Cl 1.43, 1.87), met with family less than once a month (SDC, OR 1.78 , $95 \% \mathrm{Cl} 1.40,2.27$; WP, OR 2.35, 95\% Cl 1.94, 2.84), and were less certain they could count on help from others in case of illness (WP, OR 1.23, 95\% Cl $1.08,1.41$.

Our findings suggest that persons with type 2 diabetes have poorer social relations than the general population and may need special attention to strengthening existing networks and establishing alternative networks. Social Avoidance Shows an Increased Risk for Mortality in a Type 1 Diabetes Cohort: A 22 Year Follow-Up Study

CATHERINE E. FICKLEY, TINA COSTACOU, RACHEL G. MILLER, TREVOR J. ORCHARD, Pittsburgh, PA

While both type 1 diabetes and hostility have been associated with an increased risk of mortality, whether hostility and/or its components, e.g. social avoidance (SA), increase mortality risk in type 1 diabetes is unknown.

We thus studied mortality in the prospective Pittsburgh Epidemiology of Diabetes Complications study of childhood onset type 1 diabetes $(250$ men, 256 women; mean baseline age, 29.1 and diabetes duration, 20.3 years). The Cook-Medley Hostility questionnaire was self-administered and can be broken down into 6 subscales using Barefoot Scoring methods. To determine cause of death, death certificates were obtained and any other available records or interviews were reviewed by a physician committee. Multivariable Cox proportional hazard models were used to assess independent associations between total hostility, its subscale components, and mortality.

During 22 years of follow-up, there were $128(25.3 \%)$ deaths. Only one of 6 subscales, $S A$, was univariately associated with mortality $(H R=1.09$, 1.01-1.19, $p=0.02$ ). Allowing for sex, duration, education, ever smoker, Hba1c, presence of any major diabetes complication, physical activity, Beck Depression Index (BDI), Bortner Rating Scale for type A behavior, and the other 5 hostility subscales, the significant association between SA and mortality remained $(H R=1.13,1.04-1.24, p<0.01)$. Duration, sex, smoking, presence of any major diabetes complication, $\mathrm{Hbalc}, \mathrm{BDI}$, and low type $\mathrm{A}$ behavior were also strong predictors of mortality (all $p<0.05)$. A significant interaction between $S A$ and $B D I(p<0.001)$ was demonstrated such that no significant relationship between $S A$ and mortality was found for those in the bottom two BDI quintiles (score $<7)(H R=0.94,0.81-1.08, p=0.40)$, while a positive association was seen in quintiles $3-5$ (score $\geq 7$ ) (HR=1.17, 1.05-1.31, $\mathrm{p}<0.01)$.

We conclude that in this type 1 diabetes cohort those with high SA appear to be at a higher risk of mortality, but only if their BDI score is above seven.

819-P

Reducing Diabetes Disparities: Policy Change Associated With Implementation of Interventions

NOREEN M. CLARK, MARTHA K. QUINN, BELINDA NELSON, Ann Arbor, MI

Improved services, education and support systems are required to reduce disparities in diabetes outcomes. The 5 community based sites of the Alliance to Reduce Disparities in Diabetes have introduced innovative 
interventions for those most at risk. Sites soon recognized that policy changes were necessary to move their efforts from periodic programs and projects to institutionalized ways of functioning that ensured sustainability.

This study examined policy changes initiated by Alliance sites. Data were initially collected from project leaders via a form requesting enumeration of all policy change efforts. These were examined against site documents provided routinely to the National Program Office (NPO) at the University of Michigan. A policy expert extracted confirming information from these documents, refined the original lists to include only confirmed policy changes, and elaborated to include level of change (local, citywide, statewide) and stage of accomplishment (1, beginning; 2, adoption; 3 , implementation; and 4 , full maintenance). Final lists were then verified by the Alliance teams ( $N=$ 44 individuals) in face to face meetings with the NPO leaders (N=3).

Analysis of data revealed 31 distinct policy changes initiated across the 5 sites and classified into 3 levels: 22 local, 4 citywide, 5 statewide. Of the changes, 16 were classified as being implemented or maintained. These included, for example, shaping policy decisions that led to legislation creating ACO's in New Jersey; implementation of ongoing diabetes self-management education and support in FOHCs and related networks (Chicago); creation of new job codes making community health workers part of the clinical team (Dallas); and establishing protocols for sharing patient data across clinical and community systems (Wind River Reservation). These data suggest that supportive policy changes at the local, city and state levels are fundamental to institutionalizing and sustaining interventions to reduce disparities.

Supported by: Merck Company Foundation

820-P

Correlates of Patient Assessment of Chronic Illness Care in Diabetes MARIA CHIARA ROSSI, GIACOMO VESPASIANI, SANDRO GENTILE, GIUSEPPE LUCISANO, GIAMPIERO MARRA, FABIO PELLEGRINI, BASILIO PINTAUDI, SOREN E. SKOVLUND, ANTONIO NICOLUCCI, BENCH-D AMD STUDY GROUP, Santa Maria Imbaro, Italy, San Benedetto del Tronto, Italy, Naples, Italy, Rome, Italy, Copenhagen, Denmark

The chronic care model (CCM) is receiving widespread acceptance as a standard for the delivery of diabetes care. In the context of the BENCH-D AMD study, we evaluated socio-demographic, clinical and humanistic characteristics associated with the perceived accessibility to CCM-based services.

The study was conducted in 28 diabetes outpatient clinics in Italy in the context of the DAWN-2 initiatives. In each center, a random sample of patients filled in the Patients Assessment of Chronic IIIness Care (PACIC) questionnaire. Additional instruments were: the WHO-5 well-being index, the Problem Areas in Diabetes questionnaire (PAID-5), the Health Care Climate Questionnaire (HCCO), the Patient Support (PS) questionnaire, the Diabetes Self-care Activities (DSCA) questionnaire, the Global Satisfaction for Diabetes Treatment questionnaire (GSDT) and the Diabetes Empowerment Scale (DES). Clinical data were extracted from computerized medical records.

Overall, 2434 patients with T2DM were evaluated (mean age 65.0 010.2 years, diabetes duration $13.8 \pm 15.2$ years, $59.9 \%$ males, $48.6 \%$ treated with OHA, 25.3\% treated with insulin+OHA, 24.3\% treated with insulin). When compared with patients in the lower quartile of the PACIC score, patients in the upper quartile were younger and showed significantly higher scores for WHO-5, HCCO, DES, PS, DSCA, and GSDT ( $<<0.001$ for all the scales). At multivariate analysis, adjusted for socio-demographic and clinical characteristics, the risk of presenting a PACIC score in the lowest quartile decreased for increasing values (by 5 score points) of $\mathrm{HCCO}$ (OR $0.51 ; 95 \% \mathrm{Cl}$ 0.46-0.56), GSDT (OR 0.73; 95\%Cl 0.62-0.87), DES (OR 0.85; 95\% Cl 0.770.93), WHO-5 (OR 0.96; 95\% $\mathrm{Cl} 0.94-0.99$ ) and PAID-5 (OR 0.97; 95\% $\mathrm{Cl} 0.94-$ $0.99)$

Higher perceived accessibility to CCM-based services is associated with higher levels of psychological well-being, satisfaction and perceived support, higher compliance with self-care activities, lower perception of diabetes burden, and higher levels of empowerment.

Supported by: Novo Nordisk Italy

821-P

Examining Concurrent Validity of the Mealtime BOLUS Score With Common Psychosocial Correlates of Adherence in Youth

SUSANA R. PATTON, ANNA EGAN, MICHAEL B. NELSON, COURTNEY KREUL, L.

KURT MIDYETT, MARK A. CLEMENTS, Kansas City, KS, Kansas City, MO

We have developed new methodology for calculating an adherence score based on youths' frequency of mealtime insulin bolusing (BOLUS) and demonstrated its superiority to mean frequency of daily blood glucose checks in predicting youths' HbA1c. We now examine the concurrent validity of the BOLUS based on common psychosocial correlates of adherence in youth. We hypothesize that higher youth BOLUS scores will be positively correlated with adaptive variables, such as hope and self-efficacy, and negatively correlated with reports of depressive symptoms and family conflict. A sample of 72 youths (age: $14.0 \pm 2.1$ years; HbA1c: $9.0 \pm 2.1 \%$; 40 boys) participated. Youths completed the Child Hope Scale (CHS), Self-Efficacy for Diabetes Scale (SED), Diabetes Family Conflict Scale (DFCS), and a depression screener (CESD). To calculate youths' BOLUS scores, insulin pump records were obtained for the 14-days immediately preceding form completion. Youths could earn up to 3 points/day for a mealtime bolus occurring between 0600-1000, 1100-1500 and/or 1600-2200, respectively. Pump records were independently scored for the BOLUS by 2 research team members; inter-rater reliability was 0.88 . Youths' mean BOLUS was $2.2 \pm 0.65$ (range: $0.6-3.0$ ), suggesting a moderate level of adherence. Correlations revealed a significant positive relation between youths' CHS and BOLUS scores $(r=0.27, p=0.02)$, but not with the SED ( $r=0.02, p=0.84)$. A significant negative correlation was found between youths' CESD and BOLUS scores $(r=-0.29, p=0.01)$, but not with the DFCS $(r=-$ $0.21, p=0.08$ ). Our cross-sectional results suggest that at least 2 predicted relations between youths' BOLUS scores and known psychosocial correlates of adherence exist, further strengthening the psychometric strength of the BOLUS. We are now collecting data to examine these relations prospectively to determine if youths' BOLUS can be predicted (up to 6 months) by these measures of psychosocial functioning.

Supported by: DK076921

Confirmation of Driving Risk Assessment and Risk Reduction in those With Type 1 Diabetes (T1D) through DiabetesDriving.com DANIEL COX, HARSIMRAN SINGH, TOM BANTON, DEREK FORD, LINDA GONDERFREDERICK, KAREN INGERSOLL, Charlottesville, VA, Atlanta, GA

ADA's position statement indicates there is a subset of drivers with T1D who are at an increased risk of driving mishaps. DiabetesDriving.com is a website designed to identify high-risk drivers, and deliver a 5-session personalized intervention to prevent and better detect/manage extreme blood glucose excursions while driving

416 T1D drivers from across the US have completed the online screening. using a previously-developed algorithm to determine driving risk level which identified 117 as eligible high risk and 86 as eligible low risk drivers. Participants were randomly assigned to either Routine Care (RC) or DiabetesDriving.com (DD) intervention groups. All completed on-line assessment 0 , which included 8 questions concerning preventative (e.g. How often do you measure your blood glucose within 30 minutes of starting to drive?) and 7 risky driving behaviors (e.g. While driving, how many times was your blood glucose between 50 and 69?). Participants then underwent $\mathrm{RC}$ or DD for 10 weeks, followed by post-assessment. $62 \mathrm{RC}$ and $44 \mathrm{DD}$ participants have completed post-assessment

At assessment 0, drivers classified as High risk did not differ from Low risk drivers in preventative driving behaviors $(p=.42)$, but did report more risky driving behaviors $(\mathrm{p}<$.0001). Risk scores did not correlate with preventative behaviors $(r=.04)$ but did correlate with risky driving behaviors $(r=.38)$. Additionally, DD tended to reduce the frequency of reported risky driving behaviors compared to $\mathrm{RC}$ ( $\mathrm{p}=.06$, 1-tailed). Ultimately, the study will include 480 High/Low risk drivers with a 12-month follow-up of driving mishaps.

These preliminary findings indicate that high risk T1D drivers engage in more risky, but not preventative, driving behaviors, and that DD may reduce risky driving behaviors. The ultimate test of this intervention will be the reduction of subsequent driving mishaps during follow-up.

Supported by: NIH (R01DK028288-27)

823-P

Meaningful use of EMR for Behavioral Mental Health With Diabetes Patients

JOHN F, ZREBIEC, MARILYN RITHOLZ, RICHARD DIVINCENZO, ANN GOEBELFABBRI, ANNE BUTLER, Boston, MA

Behavioral mental health (BMH) care is an essential part of multidisciplinary adult diabetes mellitus (DM) management. Given the increasing epidemic of DM in the United States, the limited BMH sessions allowed by health insurance, and the need to improve DM management, we examined Electronic Medical Records (EMR) including demographic characteristics, psychiatric diagnoses, average length of treatment, and glycemic outcomes (HbA1c) from 1/1/11-11/30/12 for type 1 and type 2 DM adult patients. Our purpose was to explore the utilization and outcomes of $\mathrm{BMH}$ care at a comprehensive adult DM center (34\% type 1, 62\% type $2 \mathrm{DM}$ ) in an urban 
area. We found that $9 \%$ of overall clinic population utilized BMH services. Major psychiatric diagnoses included: $46 \%$ depressive disorders, average $10 \mathrm{BMH}$ visits with .39\% average A1c decrease, $22 \%$ adjustment disorders, average $7 \mathrm{BMH}$ visits with $.17 \%$ average A1c decrease, $17 \%$ anxiety disorders, average $9 \mathrm{BMH}$ visits with .2\% average A1c decrease, $8 \%$ bipolar disorders, averaged $16 \mathrm{BMH}$ visits with .15\% average A1c decrease, and $6 \%$ eating disorders, average $11 \mathrm{BMH}$ visits with $.5 \%$ average A1c decrease. Our findings indicate that patients who utilized BMH visits at our comprehensive diabetes center were more likely to have type $1 \mathrm{DM}$, with $\mathrm{DM}$ duration $>10$ years, use insulin, and have depressive disorders. Importantly, all patients with psychiatric diagnoses except bipolar disorder were able to decrease their A1c levels in the usual 8-12 insurance allotted BMH sessions.

Table 1

Demographics of BMH Patients

$\mathrm{N}=735$

\begin{tabular}{ll}
\hline Patient Characteristics & $\%$ \\
\hline Type of Diabetes & 57 \\
\hline Type 1 & 41 \\
\hline Type 2 & 66 \\
\hline Female & 76 \\
\hline Non-Hispanic White & \\
\hline Age - years & 33 \\
\hline $18-40$ & 41 \\
\hline $41-60$ & 26 \\
\hline$>60$ & \\
\hline Diabetes Duration & 3 \\
\hline$<1$ year & 22 \\
\hline $1-10$ years & 30 \\
\hline $11-20$ years & 19 \\
\hline$>30$ years & 40 \\
\hline College Degree or Higher & \\
\hline Treatment - Medication & 61 \\
\hline Insulin Injection & 24 \\
\hline Insulin Pump & 7 \\
\hline Oral Medications w/o insulin & 68 \\
\hline Health Insurance & 25 \\
\hline Private & 6 \\
\hline Medicare & \\
\hline Medicaid & \\
\hline & \\
\hline
\end{tabular}

$\Delta$

824-P

Critical Conversations: Are Providers Talking About TransitionRelated Issues With Adolescents With Diabetes?

JENNIFER K. RAYMOND, DANNY C. DUKE, JONAH HARRIS, KEVIN SHIMOMAEDA, LISA SHIMOMAEDA, MICHAEL HARRIS, Denver, CO, Portland, OR

The 2011 ADA position statement recommends discussing transition with adolescents at least one year prior to transition to adult care. The recommended discussion items include psychosocial issues, mental health, complications, and sexuality. The purpose of the present study was to examine the topics discussed during a typical diabetes visit with adolescents with type 1 diabetes (T1DM). After visit forms (AVF) completed by adolescents and their providers comprised 25 different transition-related topics. AVF were completed by 42 providers and 58 youth following a routine clinic visit. Mean youth age was 15.3 years (SD $=1.7), 49 \%$ were female, $84 \%$ were Caucasian. Mean duration of diabetes was 6.6 years $(S D=4.3)$, and mean $\mathrm{HbA1c}$ was $8.7 \%$ (SD = 1.7).

Differences were identified between provider and youth responses for 5 of the 25 topics discussed. Youth endorsed greater discussion of work $(p=.020)$, insurance ( $p=.002)$, supplies/refills $(p=.006)$, eating issues/weight concerns ( $p=$ $009)$, and meal planning $(p=.029)$. The topics youth most frequently reported were physical activity $(72.4 \%)$, supplies/refills (67.2\%), meal planning (39.7\%), and complications (39.7\%). The topics providers most frequently reported were physical activity $(64.3 \%)$, supplies/refills $(39.0 \%)$, support $(38.1 \%)$, and driving $(38.1 \%)$. Topics youth reported discussing the least were anxiety $(3.4 \%)$, pregnancy $(5.2 \%)$, sexuality $(6.9 \%)$, depression $(8.6 \%)$, and diabetes burnout $(8.6 \%)$. Providers reported discussing the topics of work $(2.4 \%)$, career $(2.4 \%)$, sexuality (2.4\%), $\operatorname{ADHD}(2.4 \%)$, and pregnancy (2.4\%) the least.
These data indicate youth with T1DM and providers recall discussions around transition-related topics differently and many recommended topics are infrequently discussed. Further investigation is needed in this area, including resources to assist providers with tackling the critical health care issues during transition of care, especially mental health and high-risk behaviors.

825-P

The Feasibility of Peer Mentoring to Improve Adherence in Adolescents and Young Adults With Type 1 Diabetes

YANG LU, ELIZABETH PYATAK, ANNE L. PETERS, JAMIE R. WOOD, MICHELE KIPKE, MARISA COHEN, PAOLA A. SEOUEIRA, Los Angeles, CA

Poor glycemic control due to non-adherence is common among adolescents and young adults with Type 1 diabetes (T1D). We examined the feasibility of peer mentoring as a potential adherence-promoting intervention, as it has been effective in treating other chronic conditions. A survey was administered to 54 adolescents/prospective mentees and 46 young adults/prospective mentors $(n=100)$ enrolled at the diabetes clinics of a large pediatric hospital and a county hospital from January-September, 2012. The topics included demographics, disease history, glycemic control, adherence, depression, barriers to disease management, social support, and interest in peer mentoring. $57 \%$ of adolescents and $87 \%$ of young adults expressed interest in participating in a peer mentoring program. Stepwise multivariate logistic regressions on adolescents suggested that knowing someone else with $\mathrm{T} 1 \mathrm{D}(\mathrm{OR}=5.48, \mathrm{p}=.03)$ and wearing an insulin pump $(\mathrm{OR}=6.23, \mathrm{p}=.03)$ were positively correlated with interest in having a peer mentor. Negative correlates included younger age $(0 R=0.64, p=.06)$, living in a household smaller than five (OR=.06, $p=.01)$, and having private insurance $(\mathrm{OR}=.16, \mathrm{p}=.04)$. Among those interested in peer mentoring, phone, email, text messages, and social media were shown to be popular communication modes as well as in-person meetings. However, email was less preferred by adolescents and social media was less preferred by young adults. Among adolescents who did not want a peer mentor, $60 \%$ said they did not need help, 22\% did not feel comfortable sharing personal T1D experiences with others, and $26 \%$ had no time. Among the small fraction of young adults who did not want to be a mentor, lack of time was the major concern (66\%). In conclusion, peer mentoring is favored by the majority of adolescents and young adults with T1D in this study. A tailored program with small mentor-tomentee ratio is feasible with a very high percentage of young adults willing to serve as mentors

Supported by: NIDDK (1R03DK094513-01)

826-P

Patient Self-Care Risk Assessment in Primary Care in Trinidad and Tobago

FELICIA HILL-BRIGGS, ANDREW DHANOO, REAH SUPERVILLE, NINA SHAH, ABIGALE HARRY, AKENATH MISIR, Baltimore, MD, Port of Spain, Trinidad and Tobago

While the US is ranked $29^{\text {th }}$ in global diabetes burden, Trinidad \& Tobago (TT) is $16^{\text {th }}$ worldwide and $1^{\text {st }}$ in diabetes burden among Caribbean nations. Through a collaborative with the Ministry of Health, the study was conducted in a TT regional health centre with high diabetes prevalence to: a) test utility of integrating a Patient Self-Care Risk Assessment (PSCRA) in routine primary care and b) identify modifiable behavioral risk factors for patient self-management support interventions.

A sample of 523 patients, $98 \%$ of registered diabetes patients attending the regional health centre, consented to participation and completed the PSCRA during a routine clinic visit. Clinical data were extracted from medical records.

Participants were $63 \%$ female, age $59 \pm 11, \mathrm{HbA1c} 8.4 \pm 2.4 \%$, random blood sugar $214 \pm 86 \mathrm{mg} / \mathrm{dL}$, systolic BP $147 \pm 26 \mathrm{mmHg}$, diastolic BP 83 $\pm 15 \mathrm{mmHg}$, total cholesterol $204 \pm 49 \mathrm{mg} / \mathrm{dL}$. Integrating the PSCRA into routine visits was feasible and effective. Several risk factors for poor selfmanagement were identified, including $49 \%$ reporting depressive symptoms and an $18 \%$ clinical depression rate. Heavy smokers (>10 cigarettes/day) and alcohol abusers (>5 drinks/day) also emerged as high risk groups. One in three patients reported nonadherence to diabetes, blood pressure, or cholesterol medication. Almost all (99\%) never engage in exercise and $85 \%$ sit as their primary leisure behavior. Family-related, financial, and emotional factors were specific impediments to carrying out diabetes self-care. Participants rated themselves as confident in their ability to take action for their diabetes. However, knowledge of diabetes clinical targets and behaviors was found to be insufficient to perform self-care optimally.

The PSCRA was effective in identifying behavioral needs of the population. Findings inform design of interventions to be implemented, including a 
peer-led patient education and counseling program and a targeted case management approach for depression, smoking, and alcohol abuse.

\section{7-P}

Piloting Intensive Behavioral Health for Youth With T1DM: Avoiding Avoidable Hospitalizations

MICHAEL A. HARRIS, KIM SPIRO, MATTHEW HEYWOOD, ALYSON HATTEN, DANA HOEHN, DAVID WAGNER, Portland, OR

There is a subgroup of youth with type 1 diabetes mellitus (T1DM) that have many psychosocial challenges that put them at risk for poor metabolic control and repeated hospitalization for diabetic ketoacidosis (DKA). With intervention, these DKAs are avoidable. Behavioral health interventions (e.g., BFST) have proven effective in improving T1DM treatment adherence and metabolic control.

This pilot and feasibility study involved the implementation and evaluation of an intensive behavioral health intervention (BFST) with youth with T1DM who evidenced multiple DKAs within the past year. The intensification of the intervention involved delivery of BFST multiple times per week (in-home, in the clinic, and using mhealth) and was augmented with care coordination and case management.

Ten youth with T1DM were enrolled and treated during the course of this translational endeavor. The mean age of the youth was 14.0 years $(S D=2.9)$; the mean duration of diabetes was 6.8 years (SD $=4.1$ ); $33.3 \%$ were from single-parent homes; $73.3 \%$ were Caucasian; and the average distance from the medical center was 64.8 miles $(S D=55.2)$. The mean number of DKAs per youth for the previous year was $3.1(S D=3.9)$. The average cost of care per patient for the previous year was approximately $\$ 40,000$ US Dollars (USD) in hospitalizations due to DKA

Results revealed that the previous average $\mathrm{HbA1c}$ for the youth was $11.5 \%(S D=1.9)$. The mean $\mathrm{HbA} 1 \mathrm{c}$ value after the initiation of treatment was $10.9 \%(S D=2.1)$, and the mean number of DKA during treatment was 1.1. The average cost of care per patient due to DKA-related hospitalizations during treatment was approximately $\$ 13,000$ USD.

828-P

Relationship of Living Alone With Undiagnosed Diabetes in Japan: Toranomon Hospital Health Management Center Study YORIKO HEIANZA, YASUJI ARASE, SATORU KODAMA, SHIUN DONG HSIEH, AYUMI SUGAWARA, SHIRO TANAKA, AKIKO SUZUKI, OSAMU HANYU, KAZUMI SAITO, HITOSHI SHIMANO, SHIGEKO HARA, HIROHITO SONE, Ibaraki, Japan, Tokyo, Japan, Mito, Japan, Kyoto, Japan, Niigata, Japan

There has been evidence that living alone has the potential to cause adverse health effects. However, the association between household conditions, in particular living alone, and undiagnosed diabetes has not been clarified. We aimed to investigate whether living alone was associated with undiagnosed diabetes and whether this association could be attenuated by modifiable lifestyle habits. Studied were 8886 Japanese individuals without known diabetes. Identification of undiagnosed diabetes was made by fasting plasma glucose $\geq 7.0 \mathrm{mmol} / \mathrm{l}$ or $\mathrm{HbA1c} \geq 6.5 \%$ without a self-reported history of diabetes. Effect modification was examined by body mass index, hypertension, history of dyslipidemia, drinking habit, smoking habit, physical activity, vegetable intake, emotional stress and depressed mood. Single-living individuals ( $n=1386$ ) were not characterized by unfavorable cardiometabolic risk profiles but by unhealthy lifestyle habits. Living alone was significantly associated with undiagnosed diabetes (unadjusted odds ratio (OR) 1.38 (95\% Cl 1.04, 1.84; $p=0.027$ ). After adjustment for lifestyle factors, living alone was marginally predictive of having undiagnosed diabetes (OR 1.34 $(0.99,1.80) ; p=0.054)$. Further adjustment with all modifiable factors slightly attenuated the association $(O R 1.33(0.99,1.80) ; p=0.062)$. A significant association of living alone with undetected diabetes was particularly observed among individuals who were overweight, currently smoked, had no physical activity habit, or had any one of those 3 factors. In conclusion, the association between undiagnosed diabetes and living alone can be partially explained by modifiable lifestyle factors. Single-living individuals, especially those who did not engage in favorable lifestyle habits, were more likely to have undiagnosed diabetes and should be screened for risk of undiagnosed diabetes and/or glucose tested for the presence of diabetes.
829-P

Brazilian Version of The Problem Areas in Diabetes Scale (PAID-5): Short-Form Measure of Diabetes-Related Emotional Distress ROBERTA CARVALHO FIGUEIREDO, MAARTJE DE WIT, TIBOR R.S. HAJOS, SANDHI M BARRETO, Belo Horizonte, Brazil, Amsterdam, Netherlands

The Problem Areas in Diabetes Scale (PAID) has been recommended for identifying depression and diabetes-related distress, however, while clinically useful, wider use of the PAID may be limited by its length. Recognizing that diabetes-related distress seems to be a problem among Brazilian patients with diabetes, we aimed to validate the Brazilian version of the PAID-5 (B-PAID-5), a measure for rapid screening of diabetes-related emotional distress. The present study includes all 282 patients who participated in a cross-sectional epidemiological study was conducted among patients with diabetes and their Family Health physicians in 108 healthcare centres in Belo Horizonte, Brazil. Data were gathered by faceto-face interviews. The B-PAID- 5 total score correlated significantly with the B-PAID total score, $r=0.92, p<0.05$. Consistent with research suggesting that different aspects of diabetes-related distress are interrelated, yet distinct, the B-PAID-5 correlated positively with scores on the other problem areas: B-PAID-5/treatment, $r=0.66$; PAID-5/food, $r=0.71$; PAID-5/social support, $r=0.62$ (all $p$ values $<0.05$ ). Diagnostic accuracy of the PAID- 5 was established using receiver operating characteristic analysis. To evaluate the ability of the PAID- 5 to 'diagnose' high distress, a cut-off score of $\geq 33$ on the 20 item PAID was used. Using the optimal cut-off score of $\geq 9$ on the PAID-5, the AUC value was 0.97 (95\% Cl 0.95-0.99), while sensitivity was $91 \%$ and specificity was $95 \%$. Previous research has demonstrated that brief, verbally administered questions are effective at identifying depression in primary care. We suggest that B-PAID-5 is a reliable and valid outcome measure and could be quite useful for screening for psychological problems among Brazilian diabetic patients in order to prioritize psychosocial intervention.

Supported by: FINEP

830-P

Marriage Can Be Murder; Impact of Spousal Behaviors on A1c in Type 1 DM

BITHIKA THOMPSON, MICHAEL ELLIOTT, REBECCA PASTOR, JANET MCGILL, GARRY S. TOBIN, St. Louis, MO

Glycemic control is key to the prevention of long term complications in type 1 diabetics. However, glycemic control in practice is difficult to achieve related to both individual and social factors. The impact of spousal involvement on glycemic control is unknown. Our study aims to identify behaviors in spouses of Type 1 diabetics that are associated with better glycemic control.

Patients were selected from the Washington University Diabetes Center database at Barnes Jewish Hospital. All patients were aged 25-60, married $\geq 3$ years, and diabetic $\geq 5$ years. We created an involvement questionnaire centered on five concepts: insulin administration, blood glucose testing, meals, exercise, and emotional support. Paired involvement questionnaires were mailed to both the patient and spouse. Forty-nine couples chose to participate in the study. Responses were analyzed from both the spouse and patient and compared to the patient's average A1c values over the prior year.

Certain spousal behaviors were found to be associated with better glycemic control in adults with Type 1 diabetes. Couples who exercised together had an $\mathrm{OR}$ (odds ratio) of 3.8 for having a HgA1c less than 7. Patients who were reminded by their spouse to test their glucose had an OR of 3.5 for having a $\mathrm{HgA1} \mathrm{c}$ less than 7. Patients whose spouses encouraged them to make healthy dinner choices had an OR of 4.2 for having a $\mathrm{HgA} 1 \mathrm{c}$ of less than 7. If the spouse was familiar with the insulin regimen there was an $\mathrm{OR}$ of 4.13 for having a $\mathrm{HgAl} 1 \mathrm{c}$ less than 7 .

These findings suggest that spousal involvement improves glycemic control in the adult with Type 1 diabetes. This study was done in a specialty practice and most individuals were at baseline well controlled. The fact that areas of significance were seen in this environment suggests a benefit for other groups of diabetics.

Limitations to our study include a small sample size. Future studies should be aimed at testing this questionnaire in a larger population with the hopes of prospective training of spouses to achieve better glycemic control.

Supported by: Washington University School of Medicine 

Perspectives on Diabetes-Related Social Stigma: An Interview Study

JESSICA L. BROWNE, ADRIANA VENTURA, KYLIE MOSELY, JANE SPEIGHT, Melbourne, Australia, Sydney, Australia

While the medical and psychological impact of living with diabetes is relatively well understood, the social impact is under-investigated. Social stigma associated with diabetes was investigated in a semi-structured interview study, with indirect questioning about social experiences of living with diabetes and direct questioning focusing on perceptions / experience of stigma. Adults with type 2 diabetes (T2DM) were recruited primarily from the member register of Diabetes Australia-Vic (Victoria branch of the national peak body representing people with diabetes). To minimize bias in sample and responses, study advertisements did not refer to "stigma". Twenty-five eligible adults took part (12 women; mean age: $59 \pm 14$ years; mean diabetes duration: $7 \pm 7$ years). Interviews averaged 55 minutes. All were audio-recorded and transcriptions were imported into NVivo 10 for thematic analysis.

Findings revealed a wide spectrum of experience. Many participants reported some evidence of stigma such as negative social experiences or perceived negative judgments; a small number did not report any stigmatization. Most felt others blamed them for causing T2DM. Sources of blame included health professionals, family, and the media. Self-blame was also evident. Emotional distress was common, which some attributed, in part, to negative social judgements. Some expressed a reluctance to disclose their T2DM due to anticipated negative social or occupational consequences. Several expressed feelings of resentment about being associated with T2DM stereotypes (fat, lazy, bad diet); others felt there was truth in the stereotypes. Participants felt that type 1 diabetes did not have the negative associations that T2DM had.

It is evident that some people with T2DM experience or perceive diabetesrelated social stigma. Further research is needed to examine these issues in people with type 1 diabetes, and explore ways to measure and minimise diabetes-related stigma at the individual and societal levels.

832-P Correlates of Psychosocial Outcomes Among People With Diabetes in the DAWN2 Study

MARK F. PEYROT, KATHARINA K. BURNS, NORBERT HERMANNS, RICHARD I.G. HOLT, ANDRZEJ KOKOSZKA, MASSIMO MASSI BENEDETTI, ALEXANDER MAYOROV, NEIL MUNRO, ANTONIO NICOLUCCI, HEATHER L. STUCKEY, JOHAN WENS, ON BEHALF OF THE DAWN2 STUDY GROUP, Baltimore, MD, Edmonton, AB, Canada, Bad Mergentheim, Germany, Southampton, United Kingdom, Warsaw, Poland, Perugia, Italy, Moscow, Russian Federation, Surrey, United Kingdom, Santa Maria Imbaro, Italy, Hershey, PA, Antwerp, Belgium

The second Diabetes Attitudes, Wishes and Needs (DAWN2) study assessed self-reported psychosocial outcomes among people with diabetes in 17 countries across four continents.

In each country a sample of 500 adults, stratified by diabetes type and treatment, completed a questionnaire containing measures translated and psychometrically validated in all the languages represented in the study. The questionnaire incorporated the validated WHO-5 well-being index, WHOQOL-BREF, and the 5-item Problem Areas in Diabetes (PAID), as well as the newly developed Diabetes Impact on Life Dimensions that assessed impact ranging from very positive to very negative with no impact as the midpoint. Participants were 1368 adults with T1DM and 7228 with T2DM (1700 using no diabetes medication, 2591 using insulin, 2937 using other medications). Multiple regression analyses identified significant $(p<0.05)$ independent correlates of psychosocial outcomes.

There were significant variations in all outcomes across countries before and after adjustment for individual-level factors. After adjustment for country and demographic factors, risk factors were generally consistent across outcomes; better outcomes were associated with higher self-rated health, fewer complications, less frequent hypoglycemia, greater access to diabetes education and healthcare, less burden of diabetes and more psychosocial support from others. A few risk factors differed across outcomes. More family conflict was associated with worse quality of life and more diabetes distress, and experience of discrimination was associated with more diabetes distress. Any hypoglycemic medication was associated with more negative impact and insulin treatment was associated with higher PAID. When positive and negative impacts were analyzed separately, risk factor patterns differed. Improvements in modifiable risk factors (reductions in burden and increases in support) may lead to better psychosocial outcomes in adults with diabetes. Predictors of Short and Long-Term Weight Loss in a Community-
Based Diabetes Prevention Program: Healthy Living Partnerships to Prevent Diabetes

JEFFREY A. KATULA, LEAH L. SOUIRES, MARA Z. VITOLINS, SCOTT P. ISOM, CAROLINE S. BLACKWELL, MICHAEL S. LAWLOR, DAVID C. GOFF, Winston-Salem, NC, Aurora, CO

The purpose of this study was to examine the predictors of short (6-month) and long-term (12-month) weight loss in individuals with prediabetes that participated in the Healthy Living Partnerships to Prevent Diabetes (HELP PD) study, a community-based translation of the diabetes prevention program. Three hundred and one volunteers with prediates (Mean (SD) age $=58(9.5)$ years; $\mathrm{BMl}=32.7(4.0) \mathrm{m} / \mathrm{kg} 2$; fasting blood glucose $=105.5(11.3) \mathrm{mg} / \mathrm{dL}$ ) were randomly assigned to either a lifestyle weight loss intervention (LWL) that was delivered by community health workers (CHWs) or an enhanced usual care condition (UCC). The goal of the LWL was to induce $\geq 7 \%$ weight loss by inducing energy deficit through increased physical activity and reduced caloric intake. The LWL consisted of weekly group meetings for 6 months and monthly meetings for 18 months led by CHWs. CHWs were volunteers with well-controlled type 2 diabetes. The UCC comprised of two individual meetings with an RD and a monthly newsletter. Independent variables included physical activity, caloric intake, and variables derived from Social Cognitive theory and were assessed at baseline and 6 months. Multiple regression analyses revealed that baseline to 6 month changes in satisfaction with physical appearance (SPA; $p<.01)$, exercise self-efficacy (ESE; $p<.01)$, caloric intake $(p<.01)$, and weight loss self-efficacy (WLSE; $p<.01)$ were significant predictors of 6 -month weight change. Additionally, 6 -month changes in SPA $(p<.01)$, ESE $(p<.05)$, WLSE $(p<.05)$, and caloric intake $(p<.05)$ were significant predictors of 12 month weight change. The results of the present study provide support for a social cognitive approach to designing and implementing a diabetes prevention lifestyle weight loss program.

\section{Supported by: NIDDK (R18DK069901)}

834-P

Social-Ecological View of Diabetes Self-Care in Urban Latino Young Adults (YAs) With Type 2 Diabetes (T2D)

ELIZABETH PYATAK, DANIELLA FLORINDEZ, SERGIO SANDOVAL, SHAIN DAVIS, ANNE L. PETERS, MARC J. WEIGENSBERG, Los Angeles, CA

Although the incidence of $T 2 D$ is markedly increasing among YAs, particularly in racial/ethnic minority groups, little is known about barriers and facilitators of diabetes self-care unique to this population. To examine this issue in detail, a longitudinal qualitative study utilizing a narrative thematic approach was conducted among Latinos age 18-30 with T2D.

Eight YAs (age $20.6 \pm 3.5 \mathrm{yrs}$; A1C $7.4 \pm 1.7$; duration of T2D $3.9 \pm 4.8$ yrs) each completed 4-8 (mean 6.3) semi-structured interviews over a 2-11 month (mean 6.9) timespan. Interviews focused on barriers and facilitators to self-care stemming from intrapersonal, interpersonal, and environmental factors.

Intrapersonal barriers to self-care included significant knowledge gaps; low motivation stemming from uncertainty about the future; and an inflexible, all-or-nothing ideation of self-care. Interpersonal barriers included a social context where diabetes and its complications are seen as inevitable, an expectation to prioritize family caretaking over one's health, and embarrassment/stigma in social situations. Environmental barriers included unstable daily routines due to fluid work/school schedules, and logistical obstacles to accessing healthcare.

Intrapersonal facilitators of self-care included future goals which were incompatible with disability and integration of diabetes into one's identity. Intrapersonal facilitators included joint participation in physical activity, and modeling and support for other self-care behaviors. Environmental facilitators included economic security and family stability.

In summary, diabetes self-care in this population is strongly influenced by a social context in which diabetes is pervasive; limited resources leading to a high degree of life instability and future uncertainty; and deprioritizing self-care to meet other personal and family needs. These findings may be pertinent to consider in developing effective self-care interventions for this population.

Supported by: NIH/NCRR/NCATS (KL2RRO31991) 
835-P Framing Challenge and Resilience in Youth With Type 1 Diabetes: The Diabetes Photo Project

ASHBY F. WALKER, DESMOND A. SCHATZ, CATHRYN JOHNSON, JANET SILVERSTEIN, KATHRYN PARKER, SHANNON LYLES, ERICA DOUGHERTY, SHANNON PATRICK, ANASTASIA ALBANESE-O'NEILL, HENRY ROHRS, Mars Hill, NC, Gainesville, FL, Atlanta, GA

The incorporation of patient perspectives into disease management is consistently recognized as a valuable tool for refining care delivery and improving health outcomes. To better understand the perspectives of youth with type 1 diabetes we conducted an exploratory study where youth were provided with disposable cameras and prompted to take 5 photographs that captured what diabetes meant to them, accompanied by narratives of their photo choices. Demographic data were collected from parents to examine possible variations in representations including gender, age, disease duration, socioeconomic status (SES), race, and glycemic control $\left(\mathrm{HbA}_{1 \mathrm{C}}\right)$. Data analysis included a content analysis for photos and openended responses, a comparison of means for photo index scores, chi-square tests $\left(\chi^{2}\right)$ for categorical variables, and Pearson's correlation for numeric variables. Overall 40 youth participated ( 20 males and 20 females) with mean age of 15 years $( \pm 1.9)$ and mean disease duration of 6 years $( \pm 3.9)$. A Content analysis of photos/narratives ( $N=202$ photos) revealed 5 main types of representation categorized broadly under themes of challenge and resilience. Challenge photos included: (1) diabetes supplies as tethering (30\% of photos), (2) food as a source of frustration ( $28 \%)$, and (3) the body as territory for disease encroachment (10\%). Resilience photos included: (1) coping mechanisms (18\%) and (2) symbols of stigma resistance (12\%). Overall, these representations were consistent across demographic categories with two exceptions. Males tended to take more negative food depictions than females ( $p<.05$ for $\chi^{2}$ tests), and youth from more affluent households (who subsequently had better levels of glycemic control than poor/working class youth) were more likely to take photos of resilience ( $p<.05$ for $\chi^{2}$ tests). Our findings provide unique insight into adolescent experiences and indicate that SES may provide an advantage for affluent youth in meeting diabetesspecific challenges.

836-P

Measuring Self-Reported Medication Adherence in Diabetes: Performance of the ARMS-D and SDSCA Medications Subscale CHANDRA Y. OSBORN, LINDSAY S. MAYBERRY, JEFFREY S. GONZALEZ, KENNETH A. WALLSTON, SUNIL KRIPALANI, CECILIA QUINTERO, Nashville, TN, Bronx, NY

Suboptimal diabetes medication adherence is associated with poor health outcomes and higher health care costs. Objective measures of adherence are expensive, inappropriate for certain study designs or impractical for clinical administration. Since there is a need for valid self-report measures of medication adherence in diabetes, we evaluated the psychometric properties and predictive validity of the Adherence to Refills and Medications Scale for Diabetes (ARMS-D) and the Summary of Diabetes Self-Care Activities (SDSCA) medications subscale.

We recruited adults with type 2 diabetes ( $N=314$ ) who were prescribed oral agents/insulin to verbally complete the 11-item ARMS-D and the SDSCA medications subscale to calculate a subscale score ( 2 items) and percent adherence for the past week (1 item). The SDSCA items were collected separately for $\leq 4$ diabetes medications. A nurse collected point-of-care A1C.

Participants were age $51.8 \pm 11.7$ years; $65 \%$ female; $53 \%$ Black; $32 \%$ had <high school degree; and $45 \%$ had incomes $<\$ 10 \mathrm{~K}$. Average A1C was $8.2 \% \pm 2.2 \% ; 66 \%$ had $\mathrm{A} 1 \mathrm{C} \geq 7.0 \%$. The ARMS-D ( $\alpha=.86)$ and SDSCA medications subscale $(\alpha=.90)$ had good internal consistency reliability, and were strongly correlated when scored in the same direction $(r=.50, p<.01)$. In unadjusted and adjusted analyses, the ARMS-D, 2-item SDSCA subscale score, and 1-item SDSCA percent adherence score all predicted A1C ( $p<.05)$, but the ARMS-D provided a $13.2 \%$ increase in the variance explained over the covariates, whereas the SDSCA scores provided $8.4 \%$ and $7.4 \%$, respectively. Moreover, race, education, and income did not moderate this relationship.

The ARMS-D and SDSCA medications subscale are valid and reliable measures of adherence, predict $\mathrm{A} 1 \mathrm{C}$ and have stable performance across respondents. Research/clinical goals should determine what measure is used since the ARMS-D is most predictive of A1C and can identify areas of nonadherence for intervention, but it takes longer to administer than the SDSCA.

Supported by: NIH (UL1TR000445); NIDDK (K01DK087894 to C.Y.O.), (F32DK097880 to L.S.M.)
How can Mobile Phone Diabetes Programs Change Behavior? SHANTANU NUNDY, PATRICK HOGAN, JONATHAN J. DICK, ANNA P. GODDU, MARLA C. SOLOMON, MARSHALL H. CHIN, MONICA E. PEEK, Chicago, IL, New York, NY

Mobile phones are a promising platform for diabetes self-management support, but studies to date are largely based in research settings and lack theoretical grounding. We explored the behavioral effects of a real-world mobile phone-based diabetes self-management program.

Sixty-four adult members of a university-affiliated health plan with type 1 or type 2 diabetes were enrolled in a text message-based diabetes program called CareSmarts. Through CareSmarts, patients receive automated text messages on their personal mobile phones consisting of educational messages and reminders. They also text back self-assessments that enable nurse-case managers to provide between-visit telephone support. Surveys measuring self-care activities, self-efficacy, health beliefs, and social support were conducted at enrollment and 3 months.

The mean age of participants was $54 \pm 9$ years with $54 \%$ female and $53 \%$ African-African. The average baseline $\mathrm{HbA1c}$ was $8.1 \pm 2.3 \%$ and median time since diagnosis 6 years [range $0-34$ ]. Out of 11 self-care measures, 3 measures of medication adherence, exercise, and foot care improved from baseline to 3 months [Table]. Improvements in self-efficacy, perceived personal control, and amount of social support received were also observed.

A real-world mobile phone-based diabetes program improved self-care and multiple behavioral constructs. Targeted mobile phone-based diabetes interventions may function beyond simple reminders and address key barriers to optimal diabetes control.

\begin{tabular}{|c|c|c|c|c|c|}
\hline Domain & Scale & Measure & Baseline & $\begin{array}{c}3 \\
\text { Months }\end{array}$ & $\begin{array}{c}\mathbf{p} \\
\text { value }\end{array}$ \\
\hline \multirow[t]{12}{*}{ Self-care } & Morisky & Medication adherence (0-4) & 2.92 & 3.34 & $0.00^{\text {** }}$ \\
\hline & SDSCA & Diabetes medication ( $0-7$ days) & 5.96 & 6.05 & 0.51 \\
\hline & SDSCA & Healthful eating plan (0-7 days) & 4.59 & 5.28 & 0.10 \\
\hline & SDSCA & Personal eating plan (0-7 days) & 4.51 & 5.16 & 0.12 \\
\hline & SDSCA & $\begin{array}{l}\begin{array}{l}\text { Five+ servings of fruits and vegetables } \\
(0-7 \text { days })\end{array}\end{array}$ & 4.06 & 4.44 & 0.41 \\
\hline & SDSCA & High fat foods (0-7 days) & 2.22 & 2.56 & 0.24 \\
\hline & SDSCA & Participate in exercise session (0-7 days) & 2.74 & 3.02 & 0.39 \\
\hline & SDSCA & $\begin{array}{l}\text { At least } 30 \text { minutes of exercise }(0-7 \\
\text { days) }\end{array}$ & 2.73 & 3.41 & $0.04^{*}$ \\
\hline & SDSCA & Test blood sugar at least once (0-7 days) & 4.62 & 5.03 & 0.06 \\
\hline & SDSCA & $\begin{array}{l}\text { Test blood sugar as recommended (0-7 } \\
\text { days) }\end{array}$ & 4.06 & 4.33 & 0.40 \\
\hline & SDSCA & Foot inspection ( $0-7$ days) & 4.97 & 6.03 & $0.01^{* *}$ \\
\hline & SDSCA & Shoe inspection (0-7 days) & 2.19 & 2.17 & 0.91 \\
\hline Self-efficacy & Skaff & Self-efficacy (8-32) & 27.38 & 28.45 & $0.01^{\mathrm{k*}}$ \\
\hline \multirow{4}{*}{$\begin{array}{l}\text { Health } \\
\text { beliefs }\end{array}$} & DRHP & Long-term risks others (4-20) & 15.01 & 15.19 & 0.92 \\
\hline & DRHP & Long-term risks self (4-20) & 10.54 & 11.20 & 0.28 \\
\hline & RPS-DM & Risk knowledge (0-5) & 4.54 & 4.42 & 0.55 \\
\hline & RPS-DM & Perceived personal control (4-16) & 13.53 & 14.11 & $0.05^{*}$ \\
\hline \multirow{2}{*}{$\begin{array}{l}\text { Social } \\
\text { support }\end{array}$} & Tang & Amount of social support received (1-5) & 3.85 & 4.22 & $0.02^{*}$ \\
\hline & Tang & Satisfaction with social support (1-5) & 4.15 & 4.42 & 0.14 \\
\hline
\end{tabular}

* significant at $\mathrm{p}<0.05$; $^{* *}$ significant at $\mathrm{p}<0.01$

Morisky $=$ Morisky 4-Item Self-Report Measure of Medication; SDSCA $=$ Summary of Diabetes Self-Care Activities; Skaff $=$ Skaff Self-Efficacy Scale; DRHP = Diabetes

Related Health Problems; RPS-DM = Risk Perception Survey-Diabetes Mellitus; Tang

= Tang et al. The Diabetes Educator 2008 34: 266

838-P

Role of Astrocytes in the Memory Impairment in Diabetes Mellitus MASAHIRO OHSAWA, RIYO NAKAMURA, NOBORU INOUE, TOMOYASU MURAKAMI, HIROKI KATSU, SHOHEI YAMAMOTO, HIDEKI ONO, Nagoya, Japan

Diabetes mellitus is a risk factor for cognitive dysfunction. Several investigations have noted that many factors in the hippocampus are invalid in the cognitive dysfunction, but there are very few effective therapeutic regimens. The present study was then designed to investigate the involvement of astrocytes in the memory dysfunction of streptozotocin (STZ)-induced diabetic mice. Male 4-week-old ICR mice were used. Mice were rendered diabetic by a single injection of STZ $(200 \mathrm{mg} / \mathrm{kg}$, i.v.). The experiments were conducted 2 weeks after the STZ treatment. STZ-treated 
mice with blood glucose levels above $400 \mathrm{mg} / \mathrm{dl}$ were defined as diabetic. The protein expression was determined by the Western blot, and protein localization was determined by the immunohistochemistry. The cognitive function was tested in the novel object recognition test. In novel object recognition test, diabetic mice showed decreased exploration time in novel object as compared with non-diabetic mice, which was reversed by the repeated, but not acute, intracerebroventricular treatment with astroglial inhibitor propentofylline. In immunohistochemical study, the expression of glial fibrillary acidic protein (GFAP), a marker of astrocyte, in the hippocampus of diabetic mice was increased as compared with non-diabetic mice. Therefore, it is possible that memory impairment in diabetic mice may result from the activation of astrocyte in the hippocampus. Moreover, we observed the decreased expression of monocarboxylate transporter (MCT), especially MCT4, in hippocampus. Since MCT4 is involved in the release of lactate that is utilized in neurons as energy source from astrocytes, the memory impairment in diabetic mice may be improved by the normalization of the impaired brain neurometabolisms.

839-P

Impact of Behavioral Health on Diabetes Care in a Medicaid Population

JOHN T. DEVLIN, CATHERINE MCGUIRE, DEBORAH THAYER, ELSIE FREEMAN, Portland, ME, Augusta, ME

The Maine AHRO Multiple Chronic Conditions (MCC) project is a longitudinal study of the impact of behavioral health disorders (BH) on utilization, cost, quality of care and outcomes for long-term MaineCare (Medicaid) members with diabetes (DM) and other medical comorbidities. $50 \%$ of a baseline cohort of 63,141 members with continuous eligibility has a BH diagnosis: Substance Abuse only (SA); Mental Health only (MH), divided into serious mental illness (SMI) and non-SMl; and Dual (both $\mathrm{MH}$ and $\mathrm{SA})$. Compared to those with no $\mathrm{BH}$ disorder, $\mathrm{BH}$ groups have increased prevalence of DM, DM complications, new onset DM, and worsening DM (new / increased \# complications).

Person level factors increasing morbidity include older age, male gender, and having $\geq 2$ diabetes-unrelated chronic medical conditions. Low rates of DM interventions may be an issue: overall process measures (A1c, lipid, kidney, eye exam) were optimal in only $9 \%$; statin use was $54 \%$; use of antidiabetic agent $70 \%$. 20\% of those with DM were on atypical antipsychotics (AAP). Long-term use of AAP increased rates of new DM (by 73\%) and death (116\%). $30 \%$ of DM members on AAP had weak/absent indication raising the question of appropriateness of use. Factors contributing to morbidity include MCC, fragmentation of care, AAP use, and lack of preventive measures. Early identification/ treatment of $\mathrm{BH}$ disorders in diabetes care, integration of diabetes prevention activities into mental health systems, and better continuity of care may reduce disease burden.

\begin{tabular}{lccccc}
\hline Diabetes Outcomes according to Behavioral Health Group \\
\hline BH Group & $\begin{array}{c}\text { Prevalence of } \\
\text { DM }(\%)\end{array}$ & $\begin{array}{c}\text { DM with } \\
\text { chronic compli- } \\
\text { cations }(\%)\end{array}$ & $\begin{array}{c}\text { Rate of New } \\
\text { Onset DM } \\
(/ / 000)\end{array}$ & $\begin{array}{c}\text { Worse DM over } \\
\text { time }(\%)\end{array}$ & $\begin{array}{c}\text { Death Rate } \\
(/ 1000)\end{array}$ \\
\hline No BH & 7.3 & 50.7 & 29.8 & 34.3 & 8.3 \\
\hline Non-SMl & 10.4 & 54.1 & 38.6 & 36.5 & 8.9 \\
\hline SMl & 15.9 & 58.4 & 47.7 & 41.2 & 13.0 \\
\hline Dual MH/SA & 8.9 & 60.8 & 34.7 & 42.2 & 27.9 \\
\hline
\end{tabular}

Supported by: $A H R O$

\section{0-P}

Explanatory Factors of Emotional Diabetes Distress and Glycemic Control in Type 1 Diabetes

LENE E. JOENSEN, THOMAS ALMDAL, INGRID WILLAING, Gentofte, Denmark

The objective of this study was to investigate and compare explanatory factors of the variance in diabetes distress and $\mathrm{HbA1c}$ respectively - in patients with type 1 diabetes. We investigated the association of low social status, living without a partner, lack of social support and poor selfmanagement behaviors to poor glycemic control (HbA1c) and to emotional diabetes distress. Furthermore, we investigated the association of $\mathrm{HbA1c}$ level to the variance in emotional diabetes distress and the association of emotional, interpersonal, regimen and physician distress to the variance of HbA1c. We used a cross-sectional survey of 2419 Danish patients with type 1 diabetes and data from an electronic patient record. Data were analyzed using stepwise addition of covariates in multiple linear regression models with $\mathrm{HbA1c}$ and emotional diabetes distress as dependent variables. We included patient characteristics: sex, age, diabetes duration, complication status

For author disclosure information, see page 829 . and other chronic illness as covariates in all models. Results showed that patient characteristics, social status, cohabitation status and social support accounted for $23 \%(P<0.0001)$ of the total variance in emotional diabetes distress. Adding $\mathrm{HbA} 1 \mathrm{c}$ and self-management behaviors raised the explained component to $25 \%(\mathrm{P}<0.0001)$. Furthermore, patient characteristics, social status, cohabitation status and social support accounted for $8 \%(P<0.0001)$ of the variance in HbAlc. Adding emotional, interpersonal, regimen and physician diabetes distress covariates explained $19 \%(P<0.0001)$ and adding self-management behaviors raised it to $21 \%(\mathrm{P}<0.0001)$. Our study highlights the relationship between social factors, psychological factors and glycemic control. The study points towards a web of causes influencing psychological distress as well as glycemic control. The large unexplained component of variance calls for further research into factors explaining the variance in glycemic control and emotional diabetes distress.

841-P

Improvements in Glycemic Control are Associated With a Decline in the Severity of Depressive Symptoms in Patients With Type 2 Diabetes Mellitus (T2DM)

GRZEGORZ J. DZIDA, EDDY KARNIELI, ANNE LOUISE SVENDSEN, NORBERT HERMANNS, ON BEHALF OF THE SOLVE GROUP, Lublin, Poland, Haifa, Israel, Søborg, Denmark, Bad Mergentheim, Germany

The aim was to report on the prevalence and associations of depressive symptoms before and at 24-weeks, in patients with T2DM participating in an observational study of once-daily insulin detemir. Depressive symptoms were evaluated using the Patient Health Questionnaire (PHQ)-9 in Poland and Israel. Only Polish data is presented. The mean [ SD] PHQ-9 score decreased from 5.594 .95 at baseline to 3.253 .30 at final visit, a change of $-2.38[95 \% \mathrm{Cl}-2.73$, -2.02 ] $p<0.001$ (Table). Female gender, history of macro- or microvascular disease, HbA1c, and number of physician contacts were independently associated with moderate/severe depressive symptoms at baseline or final visit (Figure). Depressive symptoms are common in patients with T2DM, particularly in those with evidence of poor glycemic control and complications. Depression was associated with increased healthcare utilisation.

\begin{tabular}{ccc}
\hline Patient Health Questionnaire (PHO)-9 scores at pre-insulin and final visit \\
\hline Pre-Insulin $\quad$ Final & $\begin{array}{c}\text { Change - computed for } \\
\text { patients with pre-insulin } \\
\text { and final visit scores }\end{array}$
\end{tabular}

\begin{tabular}{llll}
\hline $\mathrm{PHO}$ score & $5.59[5.24 ; 5.93]$ & $3.25[3.02 ; 3.48]$ & $-2.38[-2.73 ;-2.02]$
\end{tabular}

[95\% Confidence Limits]

PHO depressive

symptom severity

\begin{tabular}{lcc}
\hline None & $424(53.9 \%)$ & $562(72.6 \%$ \\
\hline Mild & $200(25.4 \%)$ & $170(22.0 \%)$ \\
\hline Moderate & $109(13.9 \%)$ & $37(4.8 \%)$ \\
\hline Moderately severe & $41(5.2 \%)$ & $5(0.6 \%)$ \\
\hline Severe & $12(1.5 \%)$ & $0(0.0 \%)$ \\
\hline
\end{tabular}

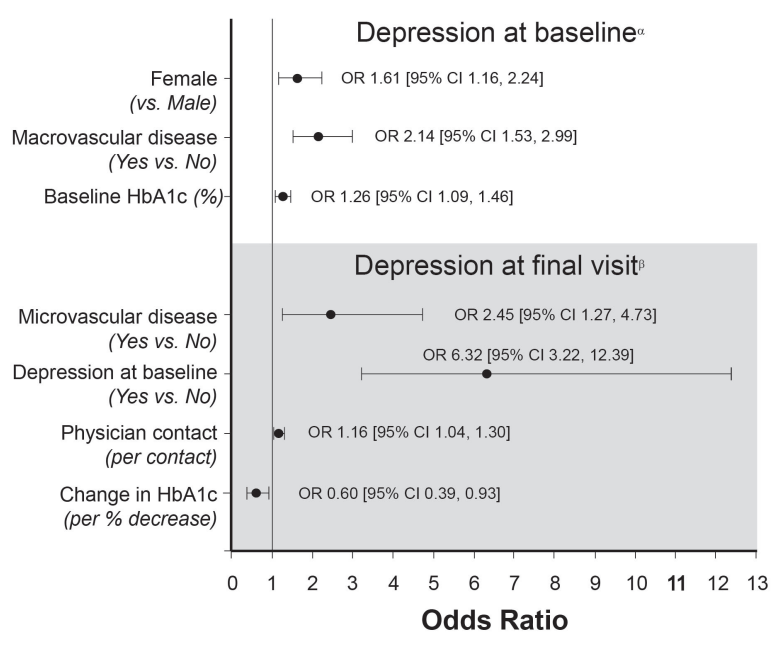

$\alpha$ - covariates included: age, sex, diabetes duration, microvascular disease (Yes/No), macrovascular disease (Yes/No), hypoglycaemia before insulin start (Yes/No), and $\mathrm{HbA1c}$ and weight at baseline $\beta$ - covariates included: age, sex, diabetes duration, microvascular disease (Yes/No), macrovascular disease (YesiNo), pre-insulin and study hypoglycaemia (both Yes/No), HbA1c and weight difference
(from baseline to final visit), contacts (sum of telephone and office contacts), and depression at baseline

Supported by: Novo Nordisk A/S

Guided Audio Tour poster

A ADA-Funded Research 
Adults With T1D: Partner Relationships and Outcomes

PAULA M. TRIEF, YAWEN JIANG, ROY BECK, DANA P. GOLDMAN, PETER J HUCKFELDT, TARA KNIGHT, KELLEE M. MILLER, RUTH S. WEINSTOCK, Syracuse, NY, Los Angeles, CA, Tampa, FL, Santa Monica, CA

Little is known about the impact of relationship status and quality on outcomes of adults with type 1 diabetes (T1D). In a subset of T1D Exchange registrants, we compared those married/partnered ( $M / P, N=1302)$ with those non- $\mathrm{M} / \mathrm{P}(\mathrm{N}=734)$. Online surveys measured relationship satisfaction (RS) and perceived partner support (active engagement (AE), protective buffering (PB), overprotection (OP)). Outcomes were clinic-reported A1c, BM and diet, exercise and blood glucose (BG) testing adherence. Pearson's chisquared test and t-test were used to compare M/Pvs. non-M/P. Multivariate regressions on normalized scales of $\mathrm{RS}, \mathrm{AE}, \mathrm{PB}$ and $\mathrm{OP}$ assessed association of relationship quality to outcomes. Compared with non-M/P participants, M/P participants were more likely to be white $(96.2 \%$ vs. $89.8 \%)$, older (mean 43.8 vs. 33.8 years), older at diagnosis (mean 20.8 vs. 15.9 years), have more education, have lower A1c (mean $7.2 \%$ vs. $7.6 \%$ ), use a continuous glucose monitor (31.4\% vs. $16.4 \%$ ) ( $p<0.01)$, and be working ( $68 \%$ vs. $32 \%$, $\mathrm{p}=0.01$ ). They were less likely to be taking medications for heart disease $(p=0.01)$, hypercholesterolemia and hypertension $(p<0.01)$ and nephropathy $(p=0.06)$. There were no differences in days with hypoglycemia or frequency of $B G$ testing. Among M/P adults, a higher level of $R S$ and $A E$ and lower level of PB and $\mathrm{OP}$ were associated with lower mean A1c after adjustment for potential confounders $(p<0.05)$. Higher $A E(p<0.01)$ and $R S(p<0.05)$ were related to lower BMI. Higher AE and lower PB were associated with diet adherence $(p<0.05)$, higher $A E$ with $B G$ testing $(p<0.01)$ and higher $R S$ with diet and exercise adherence $(p<0.001)$. In this sample of T1D Exchange registrants, being partnered was associated with lower A1c and better self-care. In those partnered, being more satisfied with one's relationship and having an actively engaged but not overprotective partner may lead to better glycemic control, weight, and self-care. Further research is warranted to better understand the role that partner relationships play in diabetes outcomes.

843-P

Early Clinical and Psychosocial Impact of First-Time Continuous Glucose Monitoring (CGM) Use

JACLYN SHEPARD, LINDA GONDER-FREDERICK, KAREN VAJDA, BORIS P. KO-

VATCHEV, Charlottesville, VA

Over the past decade, an increasing number of patients have adopted CGM for diabetes management, but little is known about their initial experiences. This study examined the clinical and psychosocial impact of CGM on individuals with no prior experience (NPE) with these devices.

As part of a parent study to design a glucose advisory system, 23 adults with type 1 diabetes for $>1$ year and use of insulin pump therapy $>6$ months who had NPE with CGM were given DexCom ${ }^{\mathrm{TM}}$ SEVEN ${ }^{\circledR}$ PLUS CGM devices for a 4-wk period. Participants were trained to use CGM but given no suggested changes in diabetes management. Pre-post assessment of BG and psychosocial variables, including hypo-/ hyperglycemia-related anxiety, and trait anxiety and depression was conducted. BG profile variables were computed for the first and last 10 days of the protocol, including the Low BG Index (LBGI), High BG Index (HBGI), overall BG Risk Index (BGRI), mean BG, and number of high and low $B G$ readings.

PairedT-testfindings indicatethatparticipants showedhigherBGRI(t=-2.99, $\mathrm{p}=.007), \mathrm{HBGI}(\mathrm{t}=-2.92, \mathrm{p}=.008)$, and average $\mathrm{BG}(\mathrm{t}=-2.39, \mathrm{p}=.007)$ during the last 10 days of the protocol. They also demonstrated an increase in trait anxiety $(t=-2.67, p=.014)$ and depression $(t=-2.55, p=.018)$. Additionally, behaviors aimed at reducing hyperglycemia decreased $(t=2.60, p=.016)$, while those aimed at avoiding hypoglycemia increased $(t=-2.08, p=$ .050) by the end of the study.

Some patients may initially exhibit negative BG profile changes with early CGM use and worsening psychosocial characteristics, including those related to hypo-/hyperglycemia-related anxiety and trait anxiety and depression. Given the rapid advancements in diabetes technology, more research in this domain is warranted.

Supported by: NIH (DK085623), (DP3DK094331)
Relationships between Depression, Anxiety, A1c, and Emotional Distress in an Adult Diabetes Population

LAUREN J. LEGATO, ELIZABETH SIMMONS, LOUIS H. PHILIPSON, TINA DROSSOS, Chicago, IL

Anxiety and depression occur more frequently in diabetic samples than in the general population, and they have also been linked to higher A1c levels. This study examined the relationships among anxiety and affective symptomatology, A1c level, and diabetes distress using brief screeners in an outpatient endocrinology clinic within an academic health center. Eightnine adult patients with Type I or II diabetes (DM) were included (age 43.1 $\pm 15.4 \mathrm{yrs}, 65 \%$ female, age at diagnosis $27.1 \pm 17.6$, diabetes duration 16.2 \pm 12.2 yrs, A1c $9.07 \pm 2.6 \%)$. Fifty-six patients (62.9\%) endorsed at least one anxiety disorder and $45(50.6 \%)$ reported depressive symptoms. Patients completed short forms of the Problem Areas in Diabetes (PAID-5) and Diabetes Distress Scale (DDS-2) as measures of emotional distress related to diabetes. Depression and anxiety were unrelated to A1c. Interestingly, depression, anxiety, and A1c were all independently, positively correlated with PAID-5 and DDS-2. In multiple regression, anxiety was predicted by higher distress scores on both the PAID-5 and DDS-2 $(F 2,86)=18.29, p<$ $\left..001, R^{2}=.295\right)$, accounting for $30 \%$ of the variance in the model. Depression was predicted by higher PAID- 5 scores $(b=.02,95 \% \mathrm{Cl}: 0.001-0.045, p=$ $.04)$, but not by DDS-2 scores. Higher A1C level was significantly predicted by higher DDS-2 scores ( $b=.29,95 \% \mathrm{Cl}: 0.032-0.546, p=.028)$, but not by the PAID-5. Results highlight the importance of assessing emotional distress through brief screeners as this may provide useful information regarding patient's mental health, specifically, anxiety and depression. Additionally, level of distress may also provide caregivers insight into factors related to poor glycemic control. Future research is needed to explore mechanisms underlying these relationships.

845-P

Central Insulin Resistance Impacts on the Serotonergic and Dopaminergic System and Causes Depressive-Like Behavior

ANDRE KLEINRIDDERS, LAURA CAPPELLUCCI, SARA VIENBERG, LAURA DARNIEDER, EMMANUEL N. POTHOS, C. RONALD KAHN, Boston, MA

Studies of type 2 diabetic patients have shown an association between diabetes and impairment in cognitive function and depression. Likewise older T2D patients show poorer performance on cognition tests compared to healthy control. Insulin action has been shown to regulate neuronal signal transmission and plasticity indicating a potential role on cognitive function. Here we investigate whether brain-specific knockout of the insulin receptor (NIRKO) in mice causes age-dependent changes in behavior as observed in diabetic patients. To test anxiety and depressive-like behavior, 10 and 17 month old NIRKO mice were subjected to mouse tail suspension, forced swimming and novelty-suppressed feeding tests. These tests showed significantly increased periods of immobility in 17 month, but not in 10 month old NIRKO mice, indicative of increased anxiety and depressive-like behavior.

There is growing evidence that the nucleus accumbens, the dopamine and serotonin system play a crucial role in the pathophysiology of depression. Examination of electrically evoked dopamine release in the nucleus accumbens revealed a decreased release of dopamine, reduced peak amplitude and width in 17 month old NIRKO mice. Even by one year of age, NIRKO mice already exhibited reduced levels of tyrosine hydroxylase, the rate limiting enzyme for dopamine synthesis, in addition to reduced expression of serotonin receptor 4, known to regulate mood and anxiety. Importantly, treatment of aged NIRKO mice with the antidepressant imipramine, which increases serotonin and dopamine action, reverses the depressive-like behavior in the mouse tail suspension test.

Thus, these data show that central insulin action modulates depressivelike behavior, presumably via serotonergic and dopaminergic signaling in the nucleus accumbens. While initially these alterations are clinically silent, with aging these result in behavioral changes and can be reversed by antidepressant treatment. 


\section{Self-Reported Diet and Exercise Unlikely to Identify Healthy Behav- iors in Type 2 Diabetes}

KAREN ADAM, HEATHER C. BRISLEN, CHRISTOPHER S. WENDEL, GREGORY FOTIEO, ELENA V. PLUMMER, JAYENDRA H. SHAH, GERALD A. CHARLTON, MARC T. MONTANARO, GLEN MURATA, Albuquerque, NM, Tucson, AZ, Phoenix, AZ

Diet, exercise, and weight control are foundations of treatment for type 2 diabetes. This study was designed to determine whether the response to standard, simple questions on lifestyle could identify patients with healthier behaviors. One hundred sixty-two patients were recruited from a clinical trial of structured SMBG at the New Mexico, Phoenix, and Southern Arizona VA medical centers. Subjects underwent a structured interview focusing on behaviors related to diabetes control. They were then asked to wear a pedometer for 7 days and keep a meal diary for $5-10$ days. The mean $( \pm$ SD) age was $64.3 \pm 8.2$ years, $97 \%$ were male, and $38 \%$ were of an ethnic minority. The average BMI was $32.9 \pm 5.4 \mathrm{~kg} / \mathrm{m}^{2}$, while HgbA1c was $8.21 \pm$ $1.24 \%$. Patients who claimed to be "on a diet" had lower daily consumption of fat $(71.5 \pm 25.8$ versus $80.8 \pm 28.9 \mathrm{gm} ; \mathrm{P}=0.04)$ and lower daily glycemic load (92.1 \pm 31.6 versus $103.9 \pm 35.4 ; P=0.034$ ) than those who did not. These differences were minor. No difference was found in caloric or carbohydrate intake, or the percent of calories from fat or carbohydrates. Of those on a diet, no difference in any nutritional parameter was found between subjects adhering to their diets "almost every day" and those who did not. Subjects who claimed to "exercise regularly" walked more than those who did not $(1.71 \pm 1.08$ versus $1.11 \pm 0.94$ miles per day, respectively; $P=0.001)$. Moreover, those who were not "physically disabled" walked more than those who were ( $1.52 \pm 1.04$ versus $1.14 \pm 0.99$ miles per day; $P=0.02)$. These differences were also minor. Hours exercised per week was derived from patient estimates of the frequency of and time per session. Simple linear regression showed no correlation between the hours reported and miles walked. For this population, simple questions similar to those asked during a clinic visit are unlikely to identify patients with healthier behaviors. Our study strongly suggests that objective assessments of nutrient consumption and activity should become part of routine care for type 2 diabetes.

Supported by: Roche Diagnostics Corporation

\section{7-P}

Identity Development and Social Support in Adolescents With Type 1 Diabetes

PERSIS V. COMMISSARIAT, JENIECE TRAST, MOLLY L. TANENBAUM, NEESHA RAMCHANDANI, RUBINA A. HEPTULLA, JEFFREY S. GONZALEZ, Bronx, NY

The purpose of the current study was to investigate the process of identity development, particularly the desire to appear "normal," in relation to peer relationships and diabetes self-management in adolescents with Type 1 diabetes (T1DM). Semi-structured qualitative interviews were coded for 27 adolescents with T1DM, ages 13 to 19, diagnosed at least 1 year prior to participation. Grounded theory and thematic content analysis were used to extract major themes in the sample. The results suggest that adolescents consistently perceived T1DM as a significant burden on their daily lives. The majority expressed a desire to feel "normal" but felt their social lives were complicated by the burden of daily T1DM self-management, particularly in social settings. However, a consistently positive effect of friend support on self-management was noted. Disclosure to friends and consistent friend support for self-management was described by nearly $60 \%$ of the sample. Those who reported accepting and incorporating T1DM into their identities reported better self-management and less difficulty in social situations. Those who minimized the role of T1DM in their identities reported poorer self-management and more difficulty in social situations. Clear gender differences were noted. Specifically, girls were more likely to incorporate their illness into their identities and report greater acceptance of T1DM. Boys were more likely to ignore self-management regimens and deny that the diagnosis of T1DM affected their identities. These results suggest that T1DM has a significant impact on adolescent social relationships and can complicate the process of identity development. Further empirical work is needed to understand how this process unfolds over time. Intervention approaches that focus on facilitating successful integration of T1DM into self-identity and support utilization of peer support hold promise in improving coping and self-management during this precarious time in development.
$\Delta$

848-P

Does Self-Esteem Mediate the Association between Personal Characteristics and Risky Behaviors in Female Adolescents With T1D? JENNIFER L. THURHEIMER, SUSAN SEREIKA, ABIGAIL WILHITE, PATRICIA SCHMITT, A. BLAIR POWELL, DENISE CHARRON-PROCHOWNIK, Pittsburgh, PA

Adolescent females with chronic illness, such as diabetes, reported lower rates of both birth control (BC) use and self-esteem (SE). Low SE is associated with risky behaviors which may result in sexually transmitted infections (STIS) and unplanned pregnancies. We examined whether SE mediated the association between personal characteristics [stage of adolescent $<15 y, 15-$ $17 y,>17 y)$, duration of illness, age of puberty] and risky behaviors/outcomes (unprotected sex, number of sex partners, STIS, BC use, alcohol, drug use) in adolescents with type 1 diabetes (T1D). Secondary analysis on baseline data from two consecutive independent randomized controlled trials evaluated efficacy of a preconception counseling (PC) intervention called READY-Girls for adolescent females with T1D. Participants from both studies $(n=141)$ completed questionnaires measuring cognitive, psychosocial, and behavioral outcomes. Items and measures were taken from the validated/published composite instrument "Reproductive Health Attitudes and Behavior Questionnaire" (RHATD), and Rosenberg's Self-Esteem Scale (possible range 10-40). All measures had Cronbach's $\alpha \geq .70$. Ages ranged 13.2-19y (mean=17y), with $4 \%(n=7)$ African American. Of the $26 \%(n=36)$ who were sexually active, (mean sexual debut $=15.6 y$ ), $64 \%$ had unprotected sex, 92\% used condoms, and $9 \%$ had an STI. Descriptive, bivariate, and multivariate analyses were performed. Mean score for SE was 31(SD=5.2; range:17-40). SE was negatively associated with drug use ( $p=.03)$ and not associated with personal characteristics. Stage of development was positively associated $(p \leq .05)$ with sexually activity and alcohol use, and negatively associated with STI. Age of puberty was negatively associated with sexual activity $(p=.04)$. SE did not have a mediating role, but predicted drug use only. In this sample, adolescents with T1D who had a younger age at puberty increased their chances for other risky behaviors and STIs.

849-P

Perspectives on T2DM from Clinicians and from People With T2DM in China: The EXPLORE Global Survey

XUEFENG YU, JOHAN WENS, ANNE M. FELTON, AYEZA BONILLA ISLAS, PETER E.H. SCHWARZ, MICHAEL E. COBBLE, Wuhan, China, Antwerp, Belgium, London, United Kingdom, Mexico City, Mexico, Dresden, Germany, Sandy, UT

The EXPLORE Global Survey examined the perceptions of clinicians (HCP) and people with Type 2 diabetes (PWD) on T2DM management. EXPLORE was an internet-based, 10-item questionnaire for PWD ( $N=2100)$ and HCPs $(\mathrm{N}=1400)$ from 13 countries. Data from $226 \mathrm{PWD}$ and $100 \mathrm{HCPs}$ from China are reported here. Differences of $\pm 3 \%$ are statistically significant and $\pm 10 \%$ meaningfully different.

There were differences in the China and global data. Fewer HCPs in China $(89 \%)$ vs globally (96\%) found managing weight (wt) the biggest challenge, perhaps because Chinese PWD were less obese (based on local definition) than global PWD. Chinese HCPs found managing microvascular complications (99\%) and CV risk (96\%) the biggest challenge; PWD were similarly concerned about long-term complications. Injectable therapy (mainly insulin) was more common in China vs globally (63 vs 33\%) and minimizing hypoglycemia was a greater challenge (79 vs $66 \%$ ).

Several disconnects exist between PWD and HCPs responses. Only $28 \%$ of PWD vs $46 \%$ of HCPs thought wt loss of $1-5 \%$ as meaningful; $71 \%$ of PWD thought wt loss of $>6 \%$ as meaningful vs $54 \%$ of HCPs. HCPs placed more emphasis on long-term wt loss; $47 \%$ (vs $26 \%$ of PWD) wanted loss for $\geq 2$ yrs. PWD perceived UTIs and yeast infections as the least acceptable therapy side-effect, while clinicians identified hypoglycemia. Interestingly, a similar number of PWD (44\%) and HCPs (40\%) would willingly accept the least acceptable side-effect, if accompanied by a $2.5 \%$ wt loss (risk/benefit trade-off). Disconnects were also noted in perceptions of emotional impact; twice as many HCPs vs PWD identified risk of CV events (29 vs $15 \%$ ) and managing blood sugar ( 31 vs $12 \%$ ) to have a negative emotional impact.

These data highlight that management of long-term complications is the most challenging T2DM issue in China. Wt management is not as highly regarded by HCPs and PWD in China compared with global results. The disconnect between PWD and HCPs views on wt loss, side-effects and emotional impact of T2DM needs to be addressed.

Supported by: Bristol-Myers Squibb/AstraZeneca 
Multilevel Interventions Can Enhance Diabetes Outcomes: Mid Term Results from the Alliance to Reduce Disparities in Diabetes MEGAN A. LEWIS, CONNIE HOBBS, SHAWN KARNS, JOSEPH BURTON, JEFFREY BRENNER, PATRIA JOHNSON, KATHY LANGWELL, MONICA PEEK, JAMES WALTON, NOREEN CLARK, DOUGLAS KAMEROW, Research Triangle Park, NC, Camden, NJ, Memphis, TN, Washakie, WY, Chicago, IL, Dallas, TX, Ann Arbor, MI

The Alliance to Reduce Disparities in Diabetes integrates innovative professional and patient education and quality of care improvements aimed at vulnerable patients, and focuses on reducing disparities in diabetes care and enhancing diabetes outcomes through clinical and community interventions. The Alliance is comprised of a National Program Office, an external evaluator, and five grantees. The five sites enrolled a multiracial patient group and implemented multilevel and multicomponent interventions to enhance patient skills, clinician cultural competencies, and health care systems changes to address disparities and enhance care. Each grantee provided clinical (hemoglobin A1c [HbA1c], blood pressure [BP]) and patient-reported outcomes (diabetes competence, quality of life, resources and supports for self-management, and diabetes self-care behaviors) for program participants to the Alliance's external evaluator, RTI International Participant cohort data show at midterm participants are $63 \%$ are female; $8 \%$ are White, 27\% African American, 3\% Native American, 54\% are Latino, $1 \%$ are Asian or Pacific Islander; and 6\% are Other or Unknown Race. 30\% are aged $18-44,34 \%$ are $45-54$ and $36 \%$ are 55 or older. Baseline and one year follow-up clinical data are reported for more than 1300 patients with Type II diabetes, with a decrease of mean $\mathrm{HbA1c}$ values from $8.5 \%$ to $7.9 \%$ and a decrease in BP from 131/80 to 130/78, both significant at the $p<.01$ level. Multivariable regression analysis also showed that patients who participated in more than half of the program had greater changes in both $\mathrm{HbA1c}$ and $\mathrm{BP}$ over time, controlling for age and gender. Analyses of the patient-reported survey measures also showed significant improvements in perceived diabetes competence, mental functioning related to quality of life, resources and supports for self-management, and self-care behaviors.

Supported by: Merck Company Foundation

851-P

Associations of Demographic Variables With Directly Observed Communication at Pediatric Visits for Type 1 Diabetes

TIM WYSOCKI, M. JOBAYER HOSSAIN, JUDITH L. ROSS, HOLLY ANTAL, LISA

BUCKLOH, ALEX TAYLOR, JENNIFER BLOSSOM, Jacksonville, FL, Wilmington, DE, Philadelphia, PA

Patient/parent demographic characteristics may affect communication with health care providers (HCP) during clinic visits. No studies have explored this in pediatric type 1 diabetes (T1D).

We analyzed baseline data from a direct observation study of communication during clinic visits for 155 youth with chronic conditions, of whom 44 have T1D. Audio-video recordings of follow-up visits for the 44 youth with T1D were coded with the Roter Interaction Analysis System (RIAS), yielding 10 communication indices per participant (interrater agreement: .84 to .93). Youth and parent age, race/ethnicity, gender, socioeconomic status (SES), and health literacy were also recorded. Results of linear mixed models analyses of demographic variables as fixed effects in relation to RIAS measures were:

HCP Communication: Only household SES was associated with HCP communication. With higher SES, HCPs asked more medical questions, made more social comments and made more frequent positive and less frequent negative comments

Parent Communication: Caucasian parents asked more medical questions and made more social and patient-activation comments. Older parents made more positive comments and parents of younger children made more emotional comments. Parents with higher health literacy scores made more social comments.

Child Communication: Caucasian youth asked more lifestyle/psychosocial questions and made more social and fewer emotional comments. Youth whose parents scored higher in health literacy offered more lifestyle/ psychosocial information. Younger children of lower SES parents made more negative comments during visits.

Significant associations with demographic variables were often for coded RIAS behaviors that occurred rarely; demographic effects were more apparent for RIAS Socio-Emotional Exchanges than for TaskOriented Exchanges. Future analysis of this complex data will explore how communication in clinic visits is associated with patient outcomes.

Supported by: Nemours Foundation
Demographic, Health and Self-Management Correlates of Personal Mastery Among Older Rural Adults With Diabetes

RONNY A. BELL, THOMAS ARCURY, SANTIAGO SALDANA, EDWARD IP, JULIENNE KIRK, JOSEPH GRZYWACZ, HA NGUYEN, SARA QUANDT, Winston-Salem, NC

Successful diabetes management requires the performance of a number of complex self-care behaviors and regular interactions with health care providers. We hypothesize that individuals who display a high degree of personal mastery may be more likely to successfully achieve selfmanagement goals. Data for this analysis come from the NIA-funded ELDER study, a cross-sectional survey examining the diabetes-related common sense models of older ( $>65$ years) African American, American Indian and white adults $(N=563)$ in rural southeastern North Carolina. Surveys were conducted by local, trained interviewers. Personal mastery was assessed using the 7-item Perlin Mastery Scale (range: 7 - 35, with higher scores indicating higher mastery levels). The instrument showed strong interna consistency in our sample $(\alpha=0.75)$. Diabetes management was assessed using the diabetes module of the Behavioral Risk Factor Surveillance System and the 11-item Summary of Diabetes Self-Care. Management indicators included personal (frequency of blood glucose-self-monitoring, foot checks, adherence to diet and exercise recommendations) and provider (foot checks, monitoring of A1c, performance of dilated eye exam, and participation in a formal diabetes education program) indicators. The mean (+SD) Mastery score for the sample was 17.6 (3.2). Demographic and health correlates of Mastery Score included: being younger than 75 , a high school education, being married, having $<3$ chronic health conditions, and having lived outside the Southern US at some point. With regard to diabetes management, Mastery scores were higher among those who reported adhering to diet and exercise recommendations, and among those who reported participating in a diabetes education program. Surprisingly, Mastery scores were higher among those who reported less frequent blood glucose self-monitoring Personal mastery may be an important factor associated with some aspects of successful diabetes management.

Supported by: R01AG17587

853-P

Long-Term Effects of Mobile Phone Based Diabetes Self-Management Education

IKUE OSAWA, MITSURU IKEDA, TOMOHIRO NABETA, TAKASHI YONEDA, YOSHIYU TAKEDA, MASUO NAKAI, MIKIYA USUKURA, KIWAMU ABE, Nomi City, Japan, Kanazawa, Japan

It is critical in diabetes self-management education to change the attitude and the behavior of patients. Mobile phone based support for them has been growing. We have already confirmed that a three month intervention by mobile phones helped patients to improve glucose metabolism, fat metabolism, and blood pressure. However, the long term effects of the support remain to be clarified. There is a possibility that such short-term mobile based education might not be sufficient for patient self-management.

The purpose of this study is to examine the persistence of the effects on patients' attitude and behavior by a previous 3 month intervention where an experimental group was promoted to photograph every single meal, record the daily number of their steps and send the data with mobile phones (Dec, 2009-March,2010). For this study (Sep, 2012), 68 participants were re-recruited from the initial experiment. The sixty-five (experimental group: 34, control group: 31) of them responded to a survey. The patient's attitude and behavior toward their self-management were compared between the control group and the experimental group using a chi-square test.

The effect on attitude toward diet of the experimental group lasted significantly higher than that of the control group $(p<0.01)$. Especially the difference was notable with women in experimental group. Furthermore effect on the distance of short range walks of the experimental group lasted significantly higher than that of the control group $(p<0.01)$.

Possible causes of these contrasts are the experimental group's experiences with the mobile phones. It is likely photographing and showing what they ate promoted the experimental group to establish a positive attitude toward their diet. Checking and transmitting the number of steps might have facilitated adherence to walking short distance in the experimental group. It is suspected the three month intervention by mobile phones is useful to keep the positive effects on patients' attitude and behavior over a long period. 
Psychosocial Support for People With Diabetes by Healthcare Providers in the DAWN2 Study

SANJAY KALRA, ANGUS FORBES, RICHARD I.G. HOLT, NEIL MUNRO, JOAO SALLES, EDELMIRO MENÉNDEZ TORRE, JOHAN WENS, INGRID WILLAING, MARK F. PEYROT, ON BEHALF OF THE DAWN2 STUDY GROUP, Karnal, India, London, United Kingdom, Southampton, United Kingdom, Surrey, United Kingdom, São Paulo, Brazil, Oviedo, Spain, Antwerpen, Belgium, Gentofte, Denmark, Baltimore, MD

In each country a sample was recruited of approximately 120 primary care physicians, 80 diabetes specialist physicians, and 80 other diabetes care providers/educators (nurses, nutritionists, etc.). Participants ( $N=4785$ ) completed a common questionnaire translated into the relevant local languages. Multiple regression analyses identified significant $(p<0.05)$ independent correlates of 2 psychosocial strategies: (a) routine provision of psychosocial assessment/support and (b) referral to mental health providers. Potential correlates included provider and practice characteristics, patient case-mix, and provider beliefs and behaviors.

There were significant cross-country variations in both psychosocial strategies after adjustment for individual-level factors. After multivariate adjustment, self-reported use of the psychosocial strategies varied by discipline/specialty and practice site, e.g., nurses and those in hospital outpatient settings were less likely than others to provide support and more likely to make a mental health referral. The strategies of psychosocial assessment/support and mental health referral were more common among HCPs who assessed depression among their patients and whose patients had higher prevalence of clinical depression, and HCPs who had been trained and appropriately resourced to provide psychosocial support, and who believed clinical success depended on dealing with emotional problems. HCPs who used these 2 psychosocial strategies wanted more training in this area.

These findings suggest that improvements in training and resources, recognition/assessment of psychosocial problems, and increased belief in the efficacy of psychosocial support may enhance HCP efforts to address psychosocial problems among adults with diabetes.

855-P

Diabetes Empowerment Council (DEC): Innovative Group Intervention for Young Adults With Type 1 Diabetes (YA-T1D) Participating in the "Let's Empower and Prepare" (LEAP) Transition Program

MARC J. WEIGENSBERG, BETH PYATAK, DANIELLA FLORINDEZ, PAOLA SEQUEIRA, JOSEPH PROVISOR, DONNA SPRUIJT-METZ, MAGALY JUAREZ, ANNE L. PETERS, Los Angeles, CA

To develop and pilot test a group intervention based on the underlying principles of Self-Determination Theory (SDT), a subgroup of 27 T1D-YA (age 19.5 .8) participating in the LEAP 1-yr transition program were offered participation in the DEC every $\sim 3$ weeks for $1.5 \mathrm{hrs}, 12$ sessions. DEC utilized the format of "Council", a facilitated group practice designed to promote trust and depth of communication, along with stress-reduction guided imagery, narrative storytelling, and simple ritual, to promote the SDT constructs of relatedness, competence, and autonomy support. Post-intervention focus group transcripts were inductively analyzed to derive themes \& develop a theoretical model of DEC process \& outcomes. Intervention changes in SDT constructs, perceived stress (PSS), and A1C were assessed. 12 participants (P) attended $\geq 1$ DEC session (mean 6.7 3.7 , range 1-11), and 15 never participated (NP) in DEC due to no childcare $(n=2)$, schedule/travel conflicts $(n=5)$, or unknown reasons $(n=8)$. Post-intervention focus group findings suggested 1) very high program acceptability; 2) increased relatedness among group members (SDT construct); 3) a theoretical model of program action. Across the intervention, there were no between-group changes in the SDT constructs of diabetes competence or autonomy support ( $p>5$ ), but PSS significantly decreased in P vs NP $(-9.0 \pm 8.5$ vs $-1.0 \pm 5.3, p<.02$; very large Effect Size $=1.2$ ). Although $P$ vs NP decreases in $A 1 C$ were not significant $(-.5 \pm 1.4$ vs $-.2 \pm .8, p=.6)$, decreased PSS predicted decreased A1C across the 1-yr intervention (linear regression, adjusted for baseline A1C \& PSS: $\beta s t d=.56 ; R 2=.19 ; p=.05)$. While substantial feasibility issues must be overcome, the DEC was well-accepted, increased relatedness among participants, and markedly reduced PSS, which may improve glycemic control. The theoretical model of program action can be tested in future studies.
Correlates of Psychosocial Outcomes among Family Members of People With Diabetes in the DAWN2 Study

JOHAN WENS, KATHARINA KOVACS BURNS, SANJAY KALRA, ANTONIO NICOLUCCI, INGRID WILLAING, MARK F. PEYROT, ON BEHALF OF THE DAWN2 STUDY GROUP, Antwerp, Belgium, Edmonton, AB, Canada, Karnal, India, Santa Maria Imbaro, Italy, Gentofte, Denmark, Baltimore, MD

About $120 \mathrm{FM}$ in each country ( $\mathrm{N}=2057)$ completed a questionnaire in local languages incorporating psychometrically validated measures and a number of new measures. FM outcomes include psychological well-being (WHO-5), quality of life (QOL-WHO-BREF) and a 1-item measure of burden of diabetes. Multiple regression analysis controlled for country and a variety of FM and PWD demographic and disease characteristics to identify aspects of living with a PWD that might be significant $(p<0.05)$ independent correlates of the outcomes.

There were statistically significant variations in all outcomes across countries before and after adjustment for individual-level factors. After adjustment for country and demographic/disease factors, significant independent psychosocial risk factors were generally consistent across outcomes. Lower levels of perceived severity of diabetes and less frustration with not knowing how to help the PWD were associated with better well-being and $\mathrm{OOL}$ and less burden. Fewer diet restrictions on FM, fewer arguments about diabetes with their PWD, and less FM responsibility for diabetes care were associated with better $\mathrm{OOL}$ and less burden. More confidence in PWD managing their own diabetes, more benefits of living with a PWD for FM health, and having found ways to help the PWD were associated with better well-being and QOL. In summary, more effective collaboration in managing diabetes was associated with more benefits, less burden, and better psychosocial outcomes.

Improvements in collaborative management of diabetes within the family may lead to better psychosocial outcomes in adult family members of people with diabetes.

857-P

Potential to Reduce Hyperglycaemia Through Peer Support in RAPSID: RAndomised Controlled Trial of Peer Support in Diabetes DAVID SIMMONS, JONATHAN GRAFFY, DAN HOLMAN, Cambridge, United Kingdom

Significant hyperglycaemia $(\mathrm{HbA1c}>8 \%, 64 \mathrm{mmol} / \mathrm{mol})$ is associated with adverse outcomes in patients with type 2 diabetes. While medications can reduce hyperglycaemia, greater adherence to medications and self management advice should also be beneficial. RAPSID is testing whether peer support can facilitate self care. We have used baseline data to assess the scope for improved self management in the RAPSID cohort.

People with Type 2 diabetes were invited into the study by poster and family practice mailout. From 20000+ invitations, 1260 consented, were eligible, completed baseline assessments and have $\mathrm{HbA1c}$ available. Mean $\mathrm{HbA1c}$ was $57 \pm 13 \mathrm{mmol} / \mathrm{mol}(7.4 \%)$, of whom $977(78 \%)$ had $\mathrm{HbA} 1 \mathrm{c}<8 \% / 64$ $\mathrm{mmol} / \mathrm{mol}$. When compared with those with a lower $\mathrm{HbA1c}$, this 'high $\mathrm{HbA1c}$ group' $(\mathrm{HiH})$ were similar in terms of age ( $65 \pm 9$ years), gender ( $60 \%$ male), ethnicity $(7.5 \%$ non-British descent) and household income. There were also no differences in past attendance for education, diabetes knowledge, current smoking, glucose monitoring, blood pressure or lipid treatment. However, HiH had diabetes longer (12 vs 9 years $p<0.001$ ), a higher BMI (33.5 vs $31.8 \mathrm{~kg} / \mathrm{m} 2 \mathrm{p}<0.001)$, were more likely to be prescribed insulin therapy ( 34 vs $12 \% \mathrm{p}<0.001$ ), less likely to receive diet alone ( 3 vs $18 \%$ ) and less likely to be satisfied with the support from friends/family. HiH were also less adherent with advice on high fat foods (eaten $3.2 \mathrm{vs} 2.8$ days/week $p=0.001$ ), physical activity (exercises 2.5 vs 3.1 days/week, $p<0.001$ ) and medication (Moriskey score 14.2 vs $14.6 p=0.001$; careless and intentional $p<0.005$ ). HiH were more depressed by $\mathrm{PHO8}$, with greater diabetes distress, lower quality of life by E05D and lower self efficacy.

We conclude that those with poorer glucose control are less adherent and could well benefit from peer support. We look forward to testing this hypothesis in RAPSID and assessing whether peer support also reduces depression, diabetes distress and improves self efficacy and E05D.

Supported by: Peers for Progress 


\section{CLINICAL THERAPEUTICS/NEW TECHNOLOGY—GLUCOSE MONITORING AND SENSING}

858-P

The Impact of a Video Phone Reminder System on Glycemic Control in Older Adults With Type 2 Diabetes Mellitus (T2DM) in a Retirement Home

LUCIA NOVAK, SUSAN WALKER, STEPHANIE FONDA, VIRGINIA SCHMIDT, ROBERT VIGERSKY, Bethesda, MD

Interactive behavior change technologies (IBCT) - web portals connecting patients to their providers and personal health information, web-based care management programs, and mobile phone applications - have been shown to be helpful for improving outcomes in diabetes. We have shown that daily, diabetes-related video-based tips and reminders, sent to patients' mobile phones, were associated with a clinically significant improvement in A1C (Bell AM et al. J Diab Sci Tech 6:310-319, 2012). We have developed a similar intervention for older adults residing in the Armed Forces Retirement Home in which the tips were tailored to the specific needs of this population and produced by providers familiar to the subjects. We conducted a prospective, randomized pilot study in subjects ages 70-89 with T2DM for 4-30 years. They were randomized to usual care (UC) or to UC plus receiving daily $15-$ 30 second video message (VM) on a bedside phone for 6 months. A1C was measured at baseline and quarterly for up to 12 months. We found that 4 of $5 \mathrm{VM}$ subjects and only 1 of $4 \mathrm{UC}$ subjects improved their A1C at 3 months. The trend continued at 6 and 9 months but dropouts prevented analysis at 12 months (Figure). These data suggest that video tips and reminders about self care management behavior may improve glycemic control in people with DM in retirement home settings.

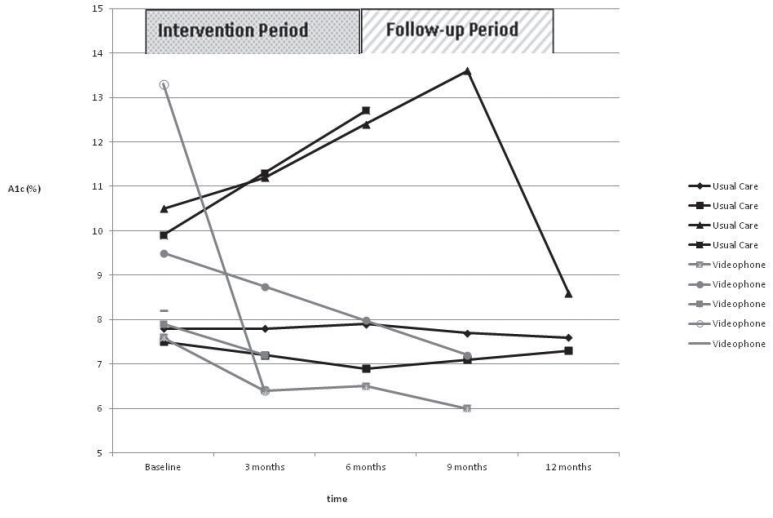

Supported by: United States Army Medical Research and Materiel Command

\section{CLINICAL THERAPEUTICS/NEW TECHNOLOGY- GLUCOSE MONITORING AND SENSING}

Guided Audio Tour: Utility of Continuous Glucose Monitoring (Posters: 859-P to $866-P)$, see page 15 .

ก 859-P

Model-Based Estimates of the Post-Prandial Response to Carbohydrate and Insulin and of the Carbohydrate-to-Insulin Ratio

FREDRIK STÅHL, ROLF JOHANSSON, ERIC RENARD, Lund, Sweden, Montpellier, France

The Carbohydrate-to-Insulin Ratio (CIR) is a measure of how many units of rapid acting insulin to administer to match the digested amount of carbohydrates, and this metric is commonly used in optimizing both the Multi-Dose Injection (MDI) and insulin pump regime of insulin dependent diabetes patients (IDDM). To estimate the value for a specific patient, a heuristic rule of dividing 500 by the Total Daily Insulin (TDI) has been suggested. This so-called 500-rule often produces poor estimates, and to improve these estimates a model-based approach is suggested.

In total, 90 patient IDDM data sets were collected in a three day hospitalized trial. The patients were equipped with Continuous Glucose Measurement systems (Abbott Freestyle), received standardized meals for breakfast, lunch and dinner (45, 70 and $70 \mathrm{gram})$, and the amount of bolus and basal doses were noted. 47 (27 pump, $20 \mathrm{MDI}$, age 42 [22-68], BMI 24.5 [16.8-35.9], HbA1c 7.8 [5.6-9.7], TDI 42 [6-82]) of the 90 data sets fulfilled quality inclusion criteria (no missing meals/insulin doses in the record, no gaps in the CGM data). For each patient data set, a segment of 24 hours was selected for model training. A linear patient-specific state-space model, combined with generic compartment models describing the insulin pharmacokinetics and the glucose rate of appearance from the gut, was identified from the CGM and meal and insulin data, using the subspace method N4SID, to describe the post-prandial impact of these inputs. The CIR of each patient was calculated by taking the ratio between the summarized amounts of carbohydrates and bolus insulin over the entire study period, and compared to the CIR estimates using the 500 rule and the model, with a correlation of 0.5 and 0.8 , respectively.

The results suggest that the suggested method could be used as a means to improve the estimates of CIR from a limited set of data.

Supported by: The European Union

ภ 860-P

Effect of Different Plasma Insulin Concentrations (PI) on Interstitial Glucose (IG): Implications for Continuous Glucose Monitoring (CGM)

PAOLO ROSSETTI, FRANCISCO JAVIER AMPUDIA-BLASCO, FÀTIMA BARCELÓRICO, JUAN F. ASCASO, JORGE BONDIA, Gandia, Spain, Valencia, Spain

Current CGM accuracy during hypoglycemia is still poor. This might be explained by insulin-induced changes of the plasma-to-interstitium glucose dynamics, not considered by CGM calibration algorithms.

The aim of the present study was to assess the role, if any, of different PI on IG.

Fourteen subjects with type 1 diabetes ( $36.5 \pm 10.5$ years, $\mathrm{HbA1c} 7.9 \pm 0.4 \%$ ) were evaluated under conditions of hyperinsulinemic eu- and hypoglycemia. Following an insulin-feedback period to standardize initial plasma glucose $(\mathrm{PG})$, each subject underwent two randomized crossover clamps with either a primed $0.3 \mathrm{mU} / \mathrm{kg} / \mathrm{min}$ (low insulin, LIS) or $1 \mathrm{mU} / \mathrm{kg} / \mathrm{min}$ (high insulin, HIS) insulin infusion. A hypoglycemic plateau of 45 minutes was achieved after a euglycemic phase, recovering later euglycemia (Figure).

Each subject wore two CGM monitors per study (Paradigm VEO, Medtronic, CA). Due to different sensor sensitivities, the raw CGM signal (nA) was normalized (CGMn) with the median pre-clamp values before statistical analysis (ANOVA)

Despite much greater mean PI concentrations in HIS vs LIS $(58.8 \pm 1.9$ vs $24.7 \pm 1.4 \mu \mathrm{U} / \mathrm{ml})$, neither PG $(79.5 \pm 4.8$ vs $80.2 \pm 4.3 \mathrm{mg} / \mathrm{dl}, \mathrm{p}=0.13)$ nor IG expressed as CGMn $(0.83 \pm 0.14$ vs $0.84 \pm 0.13, p=0.95)$ were significantly different (Figure).

In summary, differences of PI in the physiological range do not affect IG concentrations. Other factors responsible for poor CGM accuracy under hypoglycemia should be investigated.

Hyperinsulinemic, eu-hypoglycaemic clamp

A
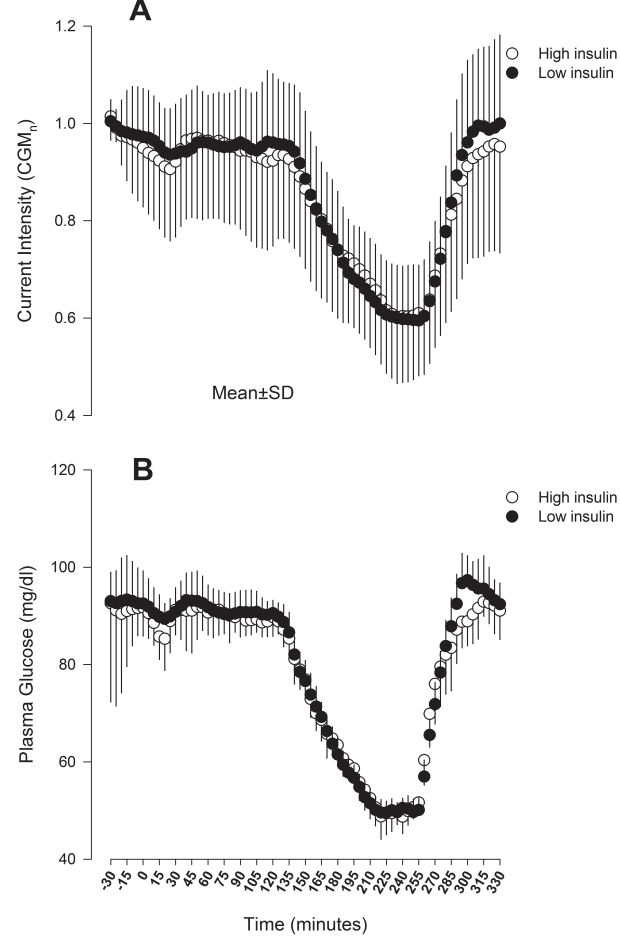

Supported by: European Union (252085); Ministry of Science (DPI2011-28112C04-01)

$\mathbf{\Delta}$ ADA-Funded Research $\Omega$ Guided Audio Tour poster

For author disclosure information, see page 829 . 